40 inting

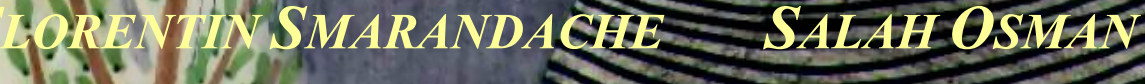

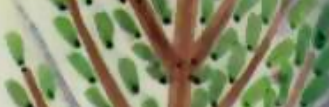

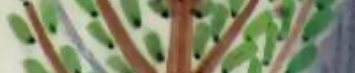

Q. $1.01 \%$

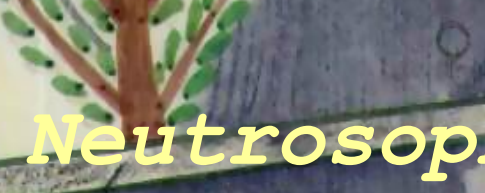

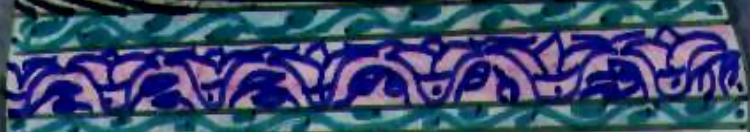

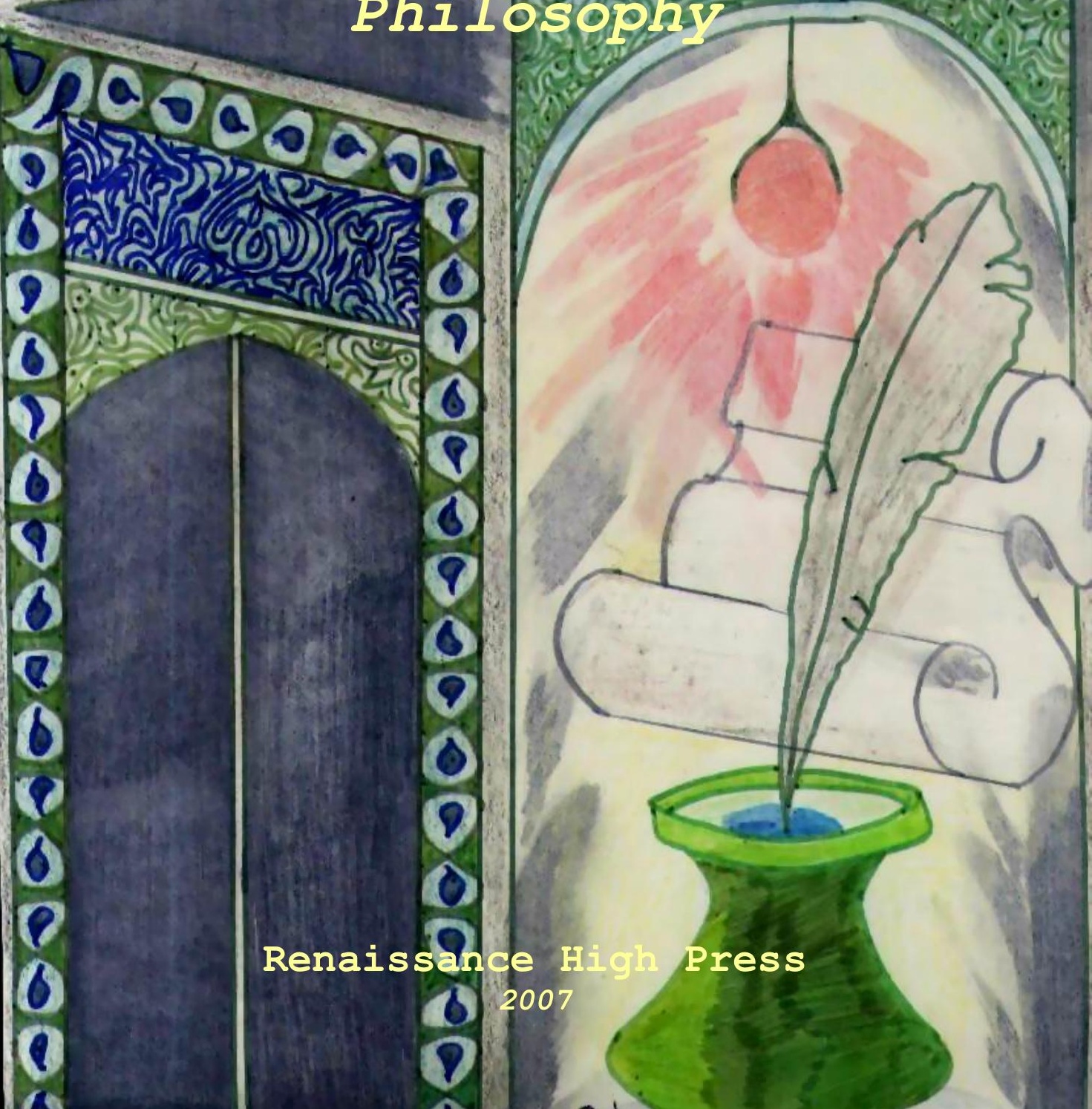

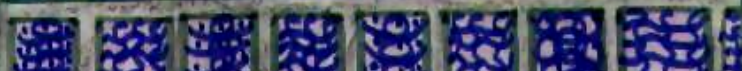

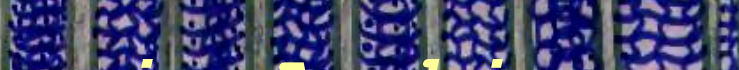
hy

Philosophy 


\section{Neutrosophy in \\ Arabic Philosophy}





\section{Neutrosophy in Arabic Philosophy}

Florentin Smarandache, $\mathcal{P h} \mathcal{D}$

Associate Professor

Chair of the Department of Math \& Sciences

University of $\mathcal{N}$ ew Mexico

200 College Rd.

Gallup, NNM 87301, USA

Salah Osman, $P h \mathscr{D}$

Assistant Professor

Department of Philosophy

Minufiya University

Faculty of Arts

She6in EIRom, Egypt

\section{Renaissance High Press}


The front cover image is done by Nada Osman, from Alexandria, Egypt, and it is titled: "From the Inspiration of Arabic Culture".

This book can be ordered in a paper bound reprint from:

\author{
Books on Demand \\ ProQuest Information \& Learning \\ (University of Microfilm International) \\ 300 N. Zeeb Road \\ P.O. Box 1346, Ann Arbor \\ MI 48106-1346, USA \\ Tel.: 1-800-521-0600 (Customer Service) \\ http://wwwlib.umi.com/bod/basic
}

Copyright 2007 by Renaissance High Press (Ann Arbor) and the Authors

Many books can be downloaded from the following

Digital Library of Science:

http://www.gallup.unm.edu/ smarandache/eBooks-otherformats.htm

Peer Reviewers:

Faculty and Students

Department of Philosophy

Minufiya University

Faculty of Arts

Shebin Elkom, Egypt

(ISBN-10): 1-931233-13-6

(ISBN-13): 978-1-931233-13-2

(EAN): 9781931233132

Printed in the United States of America 


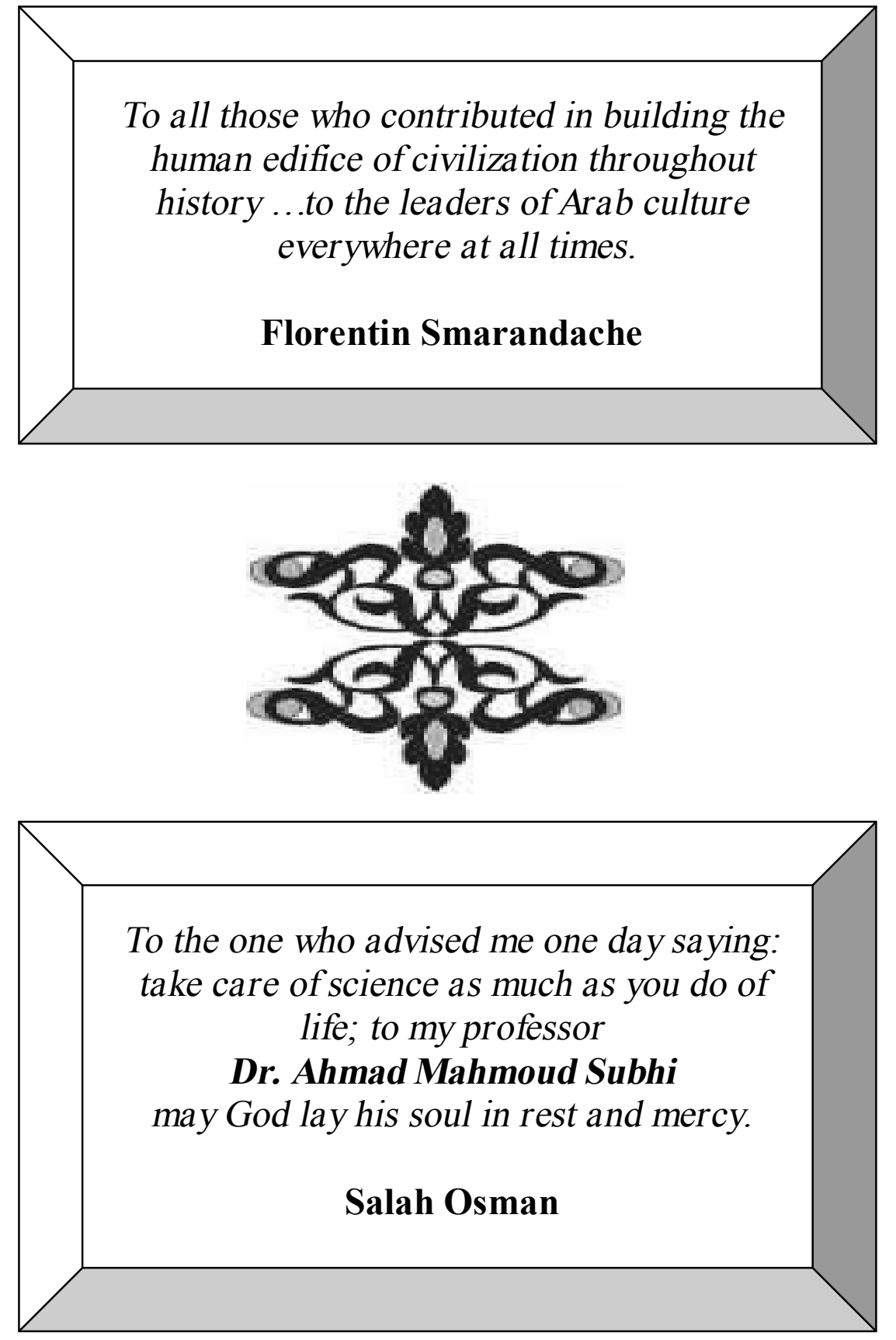





\section{Contents}

Preface

Introduction

xi

Notes of introduction

Chapter1. From Philosophy to Neutrosophy 14

1.1 Neutrosophy, a New Branch of Philosophy 14

1.2 Towards Neutrosophy (Foundamental Notions) 15

A) Etymology 15

B) Definition 15

C) Characteristics 15

D) Methods of Neutrosophic Study 16

E) Formalization 16

F) Main Principle 17

G) Fundamental thesis 17

H) Main Laws 17

I) Mottos 18

J) Fundamental Theory 18

K) Delimitation from Other Philosophical Concepts and Theories 18

L) Philosophy's Limits 19

M) Classification of Ideas 20

N) Evolution of an Idea 20

O) Philosophical Formulas 22

1.3 Neutrosophic Epistemology 28

1.4 Neutrosophic Logic 45

A) Why is the Neutrosophic Logic?

B) Definition 46

C) Differences between Neutrosophic Logic and Intuitionistic Fuzzy Logic $\quad 47$

D) The Road to the Neutrosophic Logic (a historical view) 48

E) Comments on Neutrosophic Logic in Comparison to other Logics 55

F) Comparison between Fuzzy Logic and Neutrosophic Logic 62

G) Neutrosophical Modal Logic 63

H) Applications 63 
I) Definition of Neutrosophic Logical Connectives 64

1.5 Paradoxism Used in Science 66

Notes of Chapter 1

Chapter2. Arabic-Islamic Thought: Its Sources and Pillars 72

2.1 Spatial - Temporal Dimension 72

2.2 The Neutrosophism of Designation $\quad 74$

A) Was there an Arabic Civilization before Islam? 78

B) Islamic Culture and Arabic Language $\quad 81$

C) Is It a Theological Civilization? 85

2.3 Potentials: the Positivity of Negation and Richness of Difference $\quad 90$

A) The Revelation of Heaven (Quran and Sunnah) 92

1. Quran 92

2. Sunnah 98

B) Conquests: Mixing and Absorption of the Other 103

1. Between Islam and Muslims 103

2. The Start of Combination among Nations 109

3. The Movement of Translation 110

C) The Internal Building 114

2.4 The Philosophical Cultures in Islam 121

A) The Philosophy of Islamic Philosophers 121

B) Islamic Mysticism 126

C) Ilm al-Kalam 129

D) Ilm Usul al-Fiqh (The Study of the Origins, Sources, and Principles of Islamic Jurisprudence) 134

E) Sociology or Philosophy of Politics or Philosophy ot History 139

F) The Philosophy of Grammar 141

Notes of Chapter $2 \quad 145$

Chapter3. Neutrosophic Models of the Arabic-Islamic Thought 166

3.1 A Neutrosophic Reading of the Islamic Qiyas 166

A) Meaning of Fundamental Qiyas 169

B) Cornerstones of Qiyas and its Conditions 172

a. Conditions of the Origin 174

b. Conditions of the Judgment of Origin 175

c. Conditions of the Branch 177

d. Conditions of the Cause 179

C) Paths of the Cause 184

First Path: Al-Nas (= the Text) 184

Second Path: Al-Ijma' (= the Unanimity) 186

Third Path: Al-Istinbat (= the Deduction) 186

1) Probing and Dividing 186 
2) Suitability or Al-Munasaba 188

a. The Influential Suitable 189

b. The Proper Suitable 190

c. The Free Suitable 190

3) Regularity, Retrogression, and Inseparability (Al-Tard wa al-Aks wa al- Dawaran) 190

4) Refining Reason (Tanqih al-Manat) 191

D) Types of Fundamental Qiyas 192

1) Qiyas of Priority

193

2) Qiyas of Equality 193

3) Qiyas of Minimum 194

E) Qiyas between Certainty and Probability 194

F) Ijtihad and the Renewal of Religious Thought 200

3.2 Non-Aristotelian Logical Elements in Ilm Al-Kalam 207

A) The judgment on the Perpetrator of a Major Sin 208

B) The Mental Goodness and Badness of Actions 210

C) The Somethingness of Nihil 212

D) Theory of Modes (or States) 216

Notes of Chapter $3 \quad 220$

Conclusion 236

Name Index 240

Subject Index $\quad 245$

References 256

Biography of Dr. Salah Osman 276

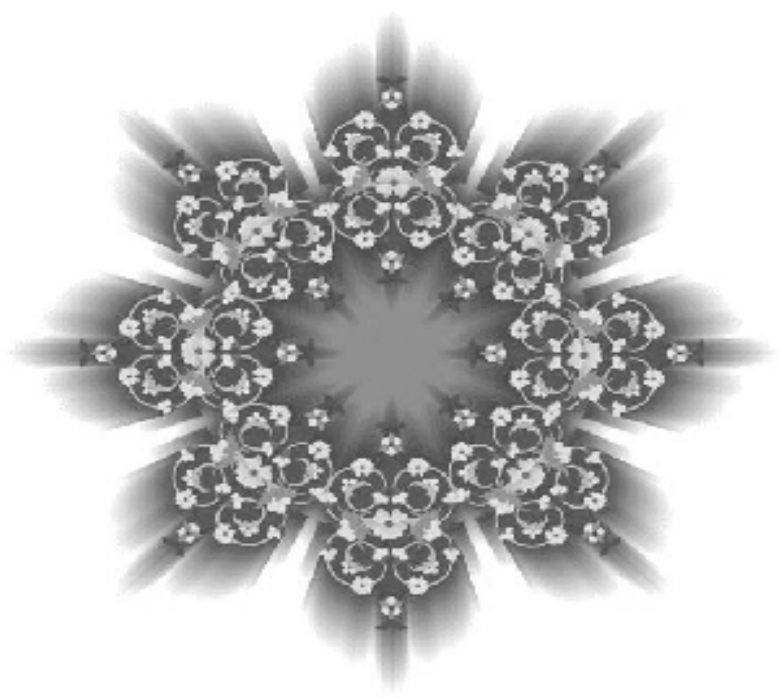





\section{Preface}

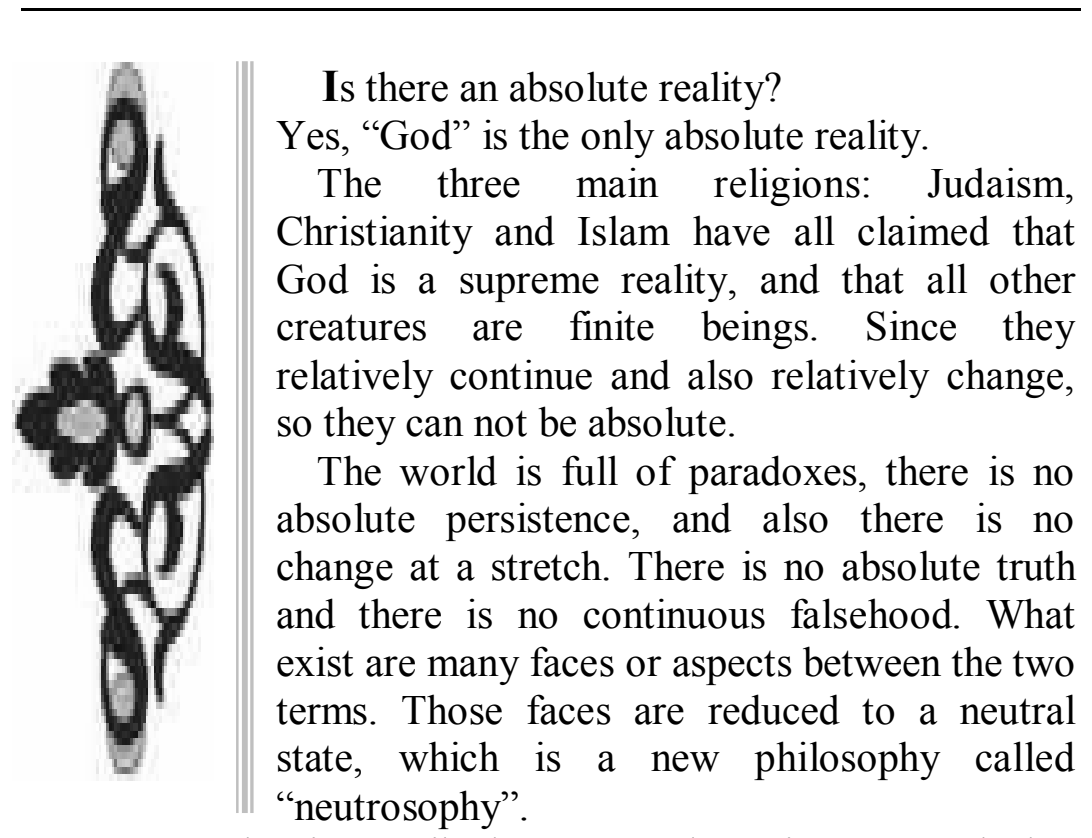

Neutrosophy is ascribed to Dr. Florentin Smarandache, professor of mathematics and sciences, and head of its department at the University of New Mexico - Gallup (USA).

The main aim of this book is to provide the reader with the philosophy of neutrosophy and its application to the ArabicIslamic thought. So the book is divided into two parts; the first belongs to prof. Smarandache, in which he exposes his philosophy of neutrosophy. The second belongs to Dr. Salah Osman, assistant professor of logic and the philosophy of science in Minufiya university - in which he applies the philosophy of neutrosophy to the Arabic-Islamic thought.

I see Smarandache's philosophy of neutrosophy not only as subjective, logical and systematic view, but also as a philosophy of his own life which he has acquired from his 
society, his relation with his native country, his studies of philosophy and mathematics and his fondness of poetry.

Personally speaking, I find the man as a sentimentalist philosopher, and also as a poet with piercing insight, so he is affected by everything in the world around us. I imagine him as a violinist playing an eternal tune.

The philosophy of neutrosophy aims at demonstrating the dialectical relation between three opposite sides: truth, falsehood, and neutral attitudes. Prof. Smarandache believes that any idea or 'thesis' contains two contrary state; the first is a proof of truth and the second is a proof of falsehood. Therefore the contradictive philosophical ideas will be transformed to consistent ideas and vise versa. Then any proposition will be explainable and accessible to all possibilities of acceptance, rejection or neutrality. This dynamic state extends through everything in the world. Things are junctive and disjunctive, hence we do not conclude the result directly, but we meet many different sorts which fluctuate between the positive and the negative. In the end we come to discover a neutral attitude.

Neutrosophy in its relation to epistemology is an analytical study shows that any idea manifests many interactions suggesting different processes which can be described in terms of three contrary concepts: thesis, antithesis and the neutral state. So the neutrosophy studies two correlated conditions:

- The conditions of the existence of the idea;

- The conditions of the impossibilities of the idea.

The historical evolution of the idea is of course an important part connected with said conditions.

Smarandache attempted to introduce a new logic called "neutrosophical logic". He suggests that it is a type of the formal logic that aims at explaining truth, falsehood, neutral propositions, and the theory of paradoxes.

In the second part of the book, Dr. Salah Osman proposes to discuss some important questions pertaining to the relation between neutrosophy and Arabic-Islamic thought since the 
first and middle ages. He is concerned to explain that the Arabic-Islamic thought includes many accurate theories and laws which organized the Islamic society. These theories and laws were earlier than the Western modern thought, consequently he exhibits a philosophical analysis of some important problems such as "is it Arabic civilization or Islamic civilization?". He also tries to connect this question or his supposition to its religious, political, historical and social origins. Then he displays the influence of these concepts on recent thought.

Salah Osman has tried to give an account of how the predecessors were aware of the logic of disagreement, and the conditions of propositions and truth in accordance with the state of space, time, variant facts and language. We therefore can deduce that the predecessors were proficient in the scholastic theology and the jurisprudence.

After Salah Osman had applied the neutrosophy to the Arabic-Islamic thought, he concluded that the Islamic jurisprudence supported most of the interpretations, and affirmed the necessity of the debates and the plurality of views.

So the major important of this book is to illustrate the debate between contemporary thinkers who try to confirm the co-operation of different cultures.

This book is published in two languages Arabic and English in both the Arab Republic of Egypt and the United State of America.

I would like very much to express my gratitude and appreciation to Prof. Florentin Smarandache and Prof. Salah Osman for having been kind to me. I have had the pleasure of reading the manuscript and of writing the preface. I do wish them all the very best.

\section{Dr. Muhammad Tawfiq al-Dawi \\ Department of Philosophy - Minufiya University Alexandria in July 2007}




\section{Introduction \\ by \\ Salah Osman}

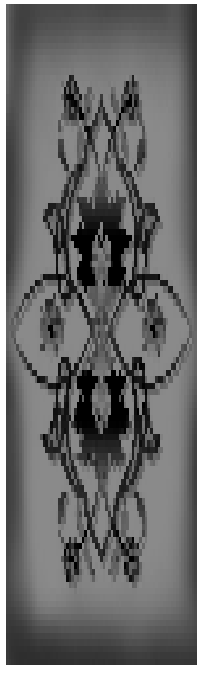

The ideas have a life filled up with activity and dynamism; a life can be likened to the swing. As soon as the idea is born, the swing carries it to and fro, from right to left and from left to right, from affirmation to negation and vice versa, from truth to falsehood and vice versa, ... etc., passing through an infinite number of cases between the two extremes, including the fixed point in the mid - distance between them. Once the speed of swing increases, and goes too far with idea the limit of truth on the one hand, and the limit of falsehood on the other, it inverted upside down, for the idea to be born anew.

Also, the most exciting feature of ideas is their capability to collaborate with opposites, and to coexist with them spatially and instantaneously. This is best demonstrated by those paradoxes, contradictions, and contrasts, which fill up our life in the various fields: philosophy, science, arts, history, politics, our cultural heritage ... etc., reaching to all positions of ordinary life. What one asserts today, another one will negate it tomorrow, and what one proves to be truthful here, another one proves to be false there. There is no fixed thing, or a permanent situation. Any truthful saying can be chased by the phantom of falsehood. Any false saying carries in its uterus the sperm of its truth proof. The only exception to this is what settle down in the heart by inspiration from God, and what the certainty in his holy books and in what honourable prophets say. 
In 1960s, Broadway theaters showed a musical comedy entitled Stop the world, I want to get off, but we must say, I think, Stop the world, I want to understand it. That is the state of human mind: an interfering intellectual spectrums; a stormy entropy, which blows up about the mental order; a whirlpool, which sucks up the views; a cyclone that goes up and down with ideas.

One preaches virtue whereas he commits the most repulsive actions!, and another advocates the sublime values while enjoying torturing his foe!. We pray God for success in committing a sin!; and we grant our money to the societies of animal kindness, whereas there are many starving people in various places in world!. We make the weapons of mass destruction to keep the peace!, and get dressed in the angel's clothes to hide an evil Satan's blemishes!; we express our gladness by shedding tears, and express our ordeal in ridiculous laughter!. Sometimes our thinkers direct us to the left, and we say Amen, other times they direct us to the right, and again we say Amen, but they go back to assert: neither left nor right, only the neutrality ..., is there another call in which we turn on one's heels according to the judgments and proofs of mind ?!.

This is the truth which the mind derives from the clamour of buffeted philosophical systems, and the noise of conflicted scientific theories, or the irrational human actions throughout history: that is, the mind never reaches by itself to the truth at all, but it will remain a prisoner of fuzzy vision. The sun rises to sets, daytime is followed by pitch-black nighttime, and successive grays. If we want a more exact philosophical understanding for this truth, we can get back to the verses of Heraclitus, even though our motives are different from his, according to the truth itself ${ }^{1}$ :

- Everything flows and nothing abides; everything gives way and nothing stays fixed.

- Cold things become warm, and what is warm cools; what is wet dries, and the parched is moistened. 
- It rests by changing.

- The death of fire is the birth of air, and death of air is the birth of water.

- It is illness that makes health sweet and good, hunger satiety, weariness rest.

- The fairest order in the world is a heap of random sweeping.

- The most handsome of apes is ugly in comparison with a human, and the wisest of men in contrast to God, appears as an ape in wisdom and beauty and every other thing.

- And it is the same thing in us that is quick and dead, awake and asleep, young and old; the former are transformed into the latter.

- Out of all the many particulars comes oneness, and out of oneness come all the many particulars.

- I was going to change my shirt, but I changed my mind instead.

Let us then rename the truth; let us call it the neutrosophy: the latest born of philosophy after hard and long parturition; and let us say that its substance is the neutrosophic logic: an update on the logical thought in its striving for grasp of reality. But what is the neutrosophy?, and what is the neutrosophic logic?. Who is the founder of the doctrine and system?, what are his motives and justifications?, and why do we shed the neutrosophic light on Arabic philosophy?.

Of course, the answers of these questions are provided in the chapters of book, but we must treat the third and fifth questions here.

$* * *$

"The Owl of Minerva", Hegel said, "first takes flight with twilight closing in". This statement expresses the tremble of mind when it collides with the irrationality of reality, or when 
it collides with a pseudo rationality by which petrified systems cover up themselves; systems seeking stability in a world changeable and speedily evolving. It is not surprising that the wisdom, as the statement points out, is sought in the pitch dark instants. That is what is reflected in the circumstances of arising out of neutrosophy and the life of its founder: the mathematician, philosopher, quantum physicist, scientific researcher in information fusion, poet, novelist, essayist, and experimental painter: Florentin Smarandache.

Smarandache is an American thinker of Romanian origin. Born on December 10, 1954, in Balcesti (district of Valcea), Romania, he graduated from the Department of Mathematics and Computer Science at the University of Craiova in 1979, and had a Ph. D. in Mathematics from the State University of Kishinev in 1997.

It is clear from the dates above that he spent his early intellectual life in the shadow of Romanian communist authority, with its totalitarianism, and the iron - hold politics of Nicolae Ceausescu (1918 - 1989), especially during the 1980s. It was natural for struggle to ensue between the regime and some of the thinkers and scientists, with Smarandache leading them. This struggle reached a climax just in 1986, when he went on a hunger strike because he was not allowed to attend the International Congress of Mathematicians at the University of Berkeley. Then he published a letter in the Notices of the American Mathematical Society about the freedom of movement of scientists. Consequently he became a dissident. As a consequence, he remained unemployed for almost two years, earning a living from private tutoring to some students.

He escaped from Romania in September 1988 and lived almost two years in the political refugee camps of Turkey, where he did unskilled labour in construction in order to survive: scavenger, house painter, whetstoner. Here he kept in touch with the French Cultural Institutes that facilitated for him accessing books and encontres with various figures. He 
left behind his peasant parents (although he was their only child), a wife expecting a child (he saw his second born son Silviu when the child was two years and a half years old, when the family reunited in the U.S.A.), and a seven year old son Mihai.

Before leaving the country he locked some of his manuscripts in a metal box in his parents' vineyard, near a peach tree. He retrieved them four years later, after the 1989 Revolution, when he returned for the first time to his native country. Other manuscripts, that he tried to mail to a translator in France, were confiscated by the secret police and never returned. He had previously tried to get some manuscripts out of Romania through the French School of Bucharest and tourists, but he couldn't locate any of them.

In March 1990, he emigrated to the United States, where he continued his postdoctoral studies at various American Universities such as University of Phoenix, University of Texas at Austin, etc. Meanwhile, he worked as a software engineer for Honeywell (1990-1995), adjunct professor for Pima Community College (1995-1997), in 1997 Assistant Professor at the University of New Mexico, Gallup Campus, where he was promoted to position of Associate Professor of Mathematics in 2003.

Since he arrived in the U.S.A., Smarandache has been very prolific. He was published so far 90 books in many fields, translated in many languages!

Also he wrote thousands of pages of unpublished diary about his life in the Romanian dictatorship, as a cooperative teacher in Morocco (Professor in Africa, 1999), in the Turkish refugee camp (Escaped... / Diary From the Refugee Camp, Vol. I, II, 1994, 1998), and in the American exile - diary, which is still going on.

But he is internationally known as the literary school pioneer for the 'paradoxism' movement which has many advocates in the world. He set up this movement in 1980, which is based on an excessive use of antitheses, antinomies, 
contradictions, paradoxes in creating works of art - both at the local level and the global level of the work of art - stablishing an interesting connection between mathematics, philosophy, and literature. He stated:

« Paradoxism started as an anti-totalitarian protest against a closed society, where the whole culture was manipulated by a small group. Only their ideas and publications counted. We couldn't publish almost anything. Then, I said: Let's do literature... without doing literature! Let's write... without actually writing anything. How? Simply: literature-object! 'The flight of a bird', for example, represents a 'natural poem', that is not necessary to write down, as it is more palpable and perceptible in any language that some signs laid on the paper, which, in fact, represent an 'artificial poem': deformed, resulting from a translation by the observant of the observed, and by translation one falsifies. 'The cars jingling on the street' was a 'disseminations poem', 'The dream with open eyes', 'a surrealist poem', 'Foolishly speaking', 'a Dadaist poem', 'The conversation in Chinese for an ignorant of this language', 'a leftist poem', 'Alternating discussions of travelers, in a train station, on different themes', 'a post - modern poem' (inter-textualism). Do you want a vertical classification?. 'Visual poem', 'Sonorous poem', 'Olfactory poem', 'Taste poem', 'Tactile poem'. Another classification is diagonal: 'poem - phenomenon', 'poem (soul) status', 'poem - thing'.

In painting and sculpture similarly - all excited in nature. Therefore, we were protesting mutely!

Later, I based it on contradictions. Why? Because we lived in that society a double life: an official one - propagated by the political system, and another one real. In mass-media it was promulgated that 'our life is wonderful', but in reality 'our life was miserable'. The paradox flourishing! And then we took the creation in derision, in inverse sense, in a syncretic way. Thus the paradoxism was born. The folk jokes, at great 
fashion in Ceausescu's 'Epoch', as an intellectual breathing, were superb springs $\gg{ }^{2}$

By means of this movement, Smarandache's contributions were plentiful in the various fields; in literature, he did many poetical experiments within his avant-garde, and edited three International Anthologies on Paradoxism (2000-2004) with texts from about 350 writers from all over the world in many languages. Also we can refer in this connection to his MetaHistory (1993), which is a theatrical trilogy against the totalitarianism again, with dramas that experiment towards a total theatre: Formation of the New Man, An Upside - Down World and The Country of Animals. The last drama, that contains no dialogue on the stage, was awarded a prize at the International Theatrical Festival of Casablanca (1995).

$\mathrm{He}$ translated them into English as A Trilogy in pARadOXisM: avant-garde political dramas; and they were published by ZayuPress (2004).

In 1999, Smarandache was nominated for the Noble Prize in Literature. Twelve books were published that analyzed his literary creation, among them: Paradoxism's Aesthetics by Titu Popescu (1995), and Paradoxism and Postmodernism by Ion Soare (2000).

In mathematics he introduced the degree of negation of an axiom or of a theorem in geometry (the Smarandache geometries, which can be partially Euclidean and partially non-Euclidean), the multi-structure (the Smarandache $\mathrm{n}$-structures, where a weak structure contains an island of a stronger structure), and multi-space (a combination of heterogeneous spaces).

He created and studied many sequences and functions in number theory. He generalized the fuzzy, intuitive, paraconsistent, multi-valent, dialetheist logics to the neutrosophic logic (also in the Denis Howe's Dictionary of Computing, England) and, similarly, he generalized the fuzzy set to the neutrosophic set (and its derivatives: paraconsistent 
set, intuitionistic set, dialethist set, paradoxist set, tautological set).

Also, he proposed an extension of the classical probability and the imprecise probability to the neutrosophic probability, which he defined as a three-dimensional vector whose components are real subsets of the non-standard interval ] $0,1^{+}[$.

On the background of this mathematical creation, he is organizing the First International Conference on Neutrosophics at the University of New Mexico,1-3 December 2001.

Since 2002, together with Dr. Jean Dezert from Office National de Recherches Aeronautiques in Paris, he worked in information fusion and generalized the Dempster-Shafer Theory to a new theory of plausible and paradoxist fusion (Dezert-Smarandache Theory).

In 2004 he designed an algorithm for the Unification of Fusion Theories and rules (UFT) used in bioinformatics, robotics, military sciences.

In physics he found a series of paradoxes (the quantum Smarandache paradoxes), and formulated the hypothesis that there is no maximal speed in the universe, which has aroused a great deal among scientists, specially as it is contradictory to Einsten's special theory of relativity. Also, he considered the possibility of a third form of matter, called unmatter, which is a combination of matter and antimatter (or quarks and antiquarks).

As for philosophy, in 1995 he introduced the concept of 'neutrosophy' ( the subject of this book ), as a generalization of Hegel's dialectic, which is the basis of his research in mathematics and economics, such as neutrosophic logic, neutrosophic set, neutrosophic probability, and neutrosophic statistics.

On the whole, we can evaluate Smarandache's works and contributions in the various fields through what was published about him; there are more than 20 books that have tackled his 
scientific activity, for example, ${ }^{3}$ : An Introduction to the Smarandache Function, by Charles Ashbacher, Vail, 1995, Computer Analysis of Number Sequences, by Henry Ibstedt, Lupton, 1998.

$* * *$

Then, why do we shed the neutrosophic light upon the Arabic philosophy? The answer of this question is restricted to three points, which themselves represent the main aims of the book.

1. Neutrosophy makes no exceptions concerning its field of study ideas. This applies to theories, principles, or concepts ... etc., of the human mind. Its main aim is showing the dialectical relation among ideas, and the potential truth, falsehood, and neutrality of ideas; hence, the potential acceptance, rejection, modification, or abolition of ideas according to the spatial and temporal variables that control the continuous process of evolution of the human mind. Because the Arabic cultural is a part and parcel of the human set of culture generally, and it has been a powerful driving force and a rich source of the modern Western culture, by means of the heritage that it preserved, and the achievements that it did in various fields during medieval era. Thus it is not to be excluded from the neutrosophic treatment. This treatment can show the right image of Arabic - Islamic thought now that it is described as closed, inflexible, petrified, refusing the other, and hostile to the dialectic dialogue, ... etc. The truth of the matter is that the Arabic - Islamic thought has always been quite the opposite of this.

2. If the power of science and its organizations led to a double ethical - ideational crisis, which the man suffers from in the present - day world, and if man conceit in his scientific 
achievements and philosophical structures has made him more arrogant; replacing his positive speculations with his religious constants, and his scientific-philosophical principles with his prophets' sayings and instructions; praying to his finite mind instead of the infinite Creator, then the neutrosophy, with its affirmation of the relativity of ideas, and the possibility of their swaying among truth, falsehood, and neutrality, also affirms that we have been in need of God, his messengers, and his heavenly books. That is what we obviously find in the Arabic philosophy's trying to achieve reconciliation between mind and revelation.

3. Thirdly and finally, the book aims to stir the contemporary Arabic mind from its dogmatic deep slumber, which hindered it from catching up with the caravan of knowledge progress, and surrounded it with the fences of positive, conclusive, and constant, ... , in a world in which the manners of change occur speedily and the divisions between many dualities stabled in our mind are falling apart. If we are to benefit from our ancestors, heritage, then it must follow their capability to break into the spectrum of grays, and recognize the significance of neutral, possible, and probable. This call can be best summarized in what Nabil Ali wrote in his Arabic culture and the age of information:

«The writer claims that one of the most important requirements of the renewal of our Arabic thought is a necessity to be in harmony with the notion of 'negation' in its wide sense, which carries in its dictionary many vocabulary items that begin with the magic and captivating syllable meaning 'No'; such as infinite, unconsciousness, non-peak, factories without human beings, offices without papers, travel in the space of information without movement, ... etc.

Also the dictionary embodies the whole of what is related to 'negation' in its meaning or significance, such as: opposition and rejection, omission and cancellation, 
changeling and against, criticism and difference, absence and nihility, randomness and chaos, jellylikeness and complexity ... etc.

We do not need to assert that our return to the jubilation of negation, and the restoration of dignity to the negation, never include any call to an anarchy, negativism, or nihilism. It aims, just in contrast with that, to development the positivism by entering the negation, not eliminating it from our invoices, i.e., by urging to break into abandoned areas, ... , passing through the grooves that separate the types of knowledge, and building the bridges among the contradictories, or what may be seen as contradictory ...

Our disdain of the 'negation', however, has resulted in the fact that we hardly accept the thought's indefiniteness, and we hardly understand many of its current problematics, nor we appreciate how the anarchy has its own theories, and the complexity has its own structure, systems, and appearances of consistency. It has led us to disapprove of the vagueness of poetry, non - objectivity of surrealistic art, non - key of serious music. We became bewildered, prisoners of the extreme simplicity, who do not comprehend many of phenomena that are opposite to the common sense, and don't submit to the direct logic of cause and effect or the mechanism of action and reaction; the phenomena which fill up our world $»{ }^{4}$

That is the same call that the book advocates: retrieval of the missing familiarity between the Arabic mind and the notion of 'negation'; and discarding the horror, which separates us from magic of metaphor, the richness of contradictions, and the productivity of difference. On the whole: unfold the neutrosophic feature of our culture and philosophy. 
In the end, I cannot but thank Allah the Almighty for His great kindness, successfulness, and charity. Also I thank my close friend Dr. Floretin Smarandache for his honest supporting to me during the stages of preparing of this book in its two versions: Arabic and English, and for his allowing me to write the introduction. Moreover, I thank him for his interest in Arabic - Islamic culture, to which he is not a stranger, because he has worked as a maths Professor in Morocco for two years, $1982-4$, teaching in the French language (which he knows well, in addition to English and Romanian ). He also reads the Quran and get familiarized with the Islamic culture, arts and literature. He has even published a Moroccan diary.

\section{Salah Osman}

Alexandria - Egypt 


\section{Notes of Introduction}

1. For an elaborate review on Heraclitus' verses in the different fields and attitudes, see: Muhammed Aly Abu Rayan, History of Greek Thought:Greek Philosophy from Thales to Plato (Tarikh al-Fikr al-Younani: al-Falsafa al-Younaniyah menn Tales ila Aflaton), The House of University Knowledge (Dar al-Ma'rifa al-Jam'iya), Alexandria, Egypt, 1988, pp. 66-72.

2. Smarandache, Florentin, http://www.gallup.unm.edu/ smarandache/

And see also: Smarandache, F., A Unifying Field in Logics: Neutrosophic Logic, Neutrosophiy, Neutrosophic Set, Neutrosophic Probability, third edition, American Research Press, 2003, Preface by Charles T. Le, pp. 3 - 4.

3. For more details about Smarandache's life and works, see: American Romanian Academy - Romanians in the Western Science and Culture, second edition, Editor Dan Grindea, Davis, CA, USA, 1996, pp. 368 - 369, also Smarandache, F., Collected Papers, Vol. II, University of Kishinev Press, Kishinev, 1997 \& The Florentin Smarandache Papers, Special Collection, Arizona State University, Hayden Library, Tempe, AZ 85287, USA .

4. Nabil Ali, Arabic Culture and the Age of Information: a View of the Future of Arabic Culture Speech (Al-Thaqafa al-Arabiyah wa Asr al-Ma'lumat), Series of the world of knowledge (Silslat A'lam al-Ma'rifa), the National Council for Culture, Arts and Literatures, Kuwait, No. 292, December 2001, pp. $204-205$.
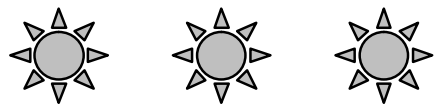


\section{Chapter 1}

\section{From Philosophy \\ To Neutrosophy ${ }^{1}$}

\subsection{Neutrosophy, a New Branch of Philosophy}

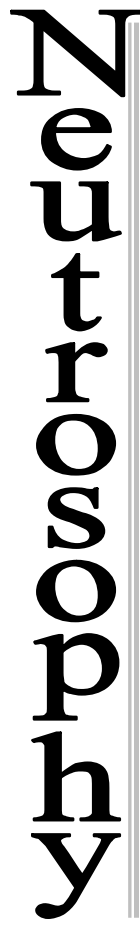

Because world is full of indeterminacy, a more precise imprecision is required. That is why, in this study, one introduces a new viewpoint in philosophy, which helps to the generalization of classical 'probability theory', 'fuzzy set' and 'fuzzy logic' to $<$ neutrosophic probability $>$, <neutrosophic set $>$ and $<$ neutrosophic logic $>$ respectively. They are useful in artificial intelligence, neural networks, evolutionary programming, neutronsophic dynamic systems, and quantum mechanics.

Especially in quantum theory, there is an uncertainty about the energy and the momentum of particles. And, because the particles in the subatomic world don't have exact positions, we better calculate their neutrosophic probabilities (i.e. involving a percent of incertitude, doubtfulness, indetermination as well - behind the percentages of truth and falsity respectively) of being at some particular points than their classical probabilities.

Besides Mathematics and Philosophy interrelationship, one searches Mathematics in connection with Psychology, Sociology, Economics, and Literature.

This is a foundation study of the NEUTROSOPHIC PHILOSOPHY because, I think, a whole collective of researchers should pass through all philosophical schools/ movements/theses/ideas and extract their positive, negative, 
and neuter features. Philosophy is subject to interpretation. This is a proppedeutique (Fr.), and a first attempt of such treatise. (An exhaustive (if possible) neutrosophic philosophy should be a synthesis of all-times philosophies inside of a neutrosophic system.).

This chapter is a collection of concise fragments, short observations, remarks, various citations, and aphorisms, some of them in a poetical form. (Main references are listed after several individual fragments.) It also introduces and explores new terms within the framework of avant-garde and experimental philosophical methods under multiple values logics.

\subsection{Towards Neutrosophy (Fundamental Notions)}

\section{A) Etymology:}

Neutro-sophy [French neutre, Latin neuter, neutral, and Greek sophia, skill / wisdom] means knowledge of neutral thought.

\section{B) Definition:}

Neutrosophy is a new branch of philosophy, which studies the origin, nature, and scope of neutralities, as well as their interactions with different ideational spectra.

\section{C) Characteristics:}

This mode of thinking:

- proposes new philosophical theses, principles, laws, methods, formulas, movements;

- reveals that world is full of indeterminacy;

- interprets the uninterpretable; regards, from many different angles, old concepts, systems:

- showing that an idea, which is true in a given referential system, may be false in another one, and vice versa;

- attempts to make peace in the war of ideas, and to make 
war in the peaceful ideas;

- measures the stability of unstable systems, and instability of stable systems.

\section{D) Methods of Neutrosophic Study:}

Mathematization (neutrosophic logic, neutrosophic probability and statistics, duality), generalization, complementarity, contradiction, paradox, tautology, analogy, reinterpretation, combination, interference, aphoristic, linguistic, transdisciplinarity.

\section{E) Formalization:}

Let's note by $<\mathrm{A}>$ an idea, or proposition, theory, event, concept, entity, by $<$ Non-A $>$ what is not $<\mathrm{A}>$, and by $<$ Anti$A>$ the opposite of $<A>$. Also, $<$ Neut-A $>$ means what is neither $<\mathrm{A}>$ nor $<$ Anti-A $>$, i.e. neutrality in between the two extremes. And $<\mathrm{A}^{\prime}>$ a version of $<\mathrm{A}>$.

$<$ Non-A $>$ is different from $<$ Anti-A $>$.

For example:

If $<\mathrm{A}>=$ white, then $<$ Anti- $\mathrm{A}>=$ black (antonym), but $<$ Non-A $>=$ green, red, blue, yellow, black, etc. (any color, except white), while $<$ Neut-A $>=$ green, red, blue, yellow, etc. (any color, except white and black), and $\left\langle\mathrm{A}^{\prime}\right\rangle=$ dark white, etc. (any shade of white).

In a classical way:

$<$ Neut-A $>\equiv<$ Neut-(Anti-A) $>$, neutralities of $<\mathrm{A}>$ are identical with neutralities of $<$ Anti-A $>$, also:

$$
\begin{gathered}
<\text { Non-A }>\supset<\text { Anti-A }> \\
\text { And } \\
<\text { Non-A }>\supset<\text { Neut-A }> \\
\text { As well } \\
<\text { A }>\cap<\text { Anti-A }>=\varnothing \\
<\text { A }>\cap<\text { Non-A }>=\varnothing
\end{gathered}
$$


$<\mathrm{A}>,<$ Neut-A $>$, and $<$ Anti-A $>$ are disjoint two by two. $<$ Non-A $>$ is the completeness of $<\mathrm{A}>$ with respect to the universal set.

But, since in many cases the borders between notions are vague, imprecise, it is possible that $\langle\mathrm{A}\rangle,\langle$ Neut- $\mathrm{A}\rangle$, $<$ Anti-A $>$ (and $<$ Non-A $>$ of course) have common parts two by two.

\section{F) Main Principle:}

Between an idea $\langle\mathrm{A}>$ and its opposite $<$ Anti-A $>$, there is a continuum-power spectrum of neutralities $<$ Neut-A $>$.

\section{G)Fundamental Thesis:}

Any idea $<\mathrm{A}>$ is $\mathrm{T} \%$ true, $\mathrm{I} \%$ indeterminate, and $\mathrm{F} \%$ false, where $\mathrm{T}, \mathrm{I}, \mathrm{F} \subset]^{-} 0,1^{+}[$.

\section{H)Main Laws:}

Let $\langle\alpha\rangle$ be an attribute, and $(\mathrm{T}, \mathrm{I}, \mathrm{F}) \subset]^{-} 0,1^{+}\left[^{3}\right.$. Then:

- There is a proposition $<\mathrm{P}>$ and a referential system $\{\mathrm{R}\}$, such that $\langle\mathrm{P}>$ is $\mathrm{T} \%\langle\alpha\rangle, \mathrm{I} \%$ indeterminate or $\langle$ Neut $-\alpha\rangle$, and $\mathrm{F} \%<$ Anti- $\alpha>$.

- For any proposition $<\mathrm{P}>$, there is a referential system $\{\mathrm{R}\}$, such that $\langle\mathrm{P}>$ is $\mathrm{T} \%\langle\alpha\rangle, \mathrm{I} \%$ indeterminate or $\langle$ Neut $-\alpha\rangle$, and $\mathrm{F} \%<$ Anti- $\alpha>$.

$-\langle\alpha\rangle$ is at some degree $\langle$ Anti- $\alpha\rangle$, while $\langle$ Anti- $\alpha\rangle$ is at some degree $\langle\alpha\rangle$.

Therefore:

For each proposition $<\mathrm{P}>$ there are referential systems $\left\{\mathrm{R}_{1}\right\},\left\{\mathrm{R}_{2}\right\} \ldots$ so that $<\mathrm{P}>$ looks differently in each of them getting all possible states from $<\mathrm{P}>$ to $<$ Non-P $>$ until $<$ Anti-P $>$.

And, as a consequence, for any two propositions $<\mathrm{M}>$ and $<\mathrm{N}>$, there exist two referential systems $\left\{\mathrm{R}_{\mathrm{M}}\right\}$ and $\left\{\mathrm{R}_{\mathrm{N}}\right\}$ respectively, such that $<\mathrm{M}>$ and $<\mathrm{N}>$ look the same. The 
referential systems are like mirrors of various curvatures reflecting the propositions.

\section{I) Mottos:}

- All is possible, the impossible too!

- Nothing is perfect, not even the perfect!

\section{J) Fundamental Theory:}

Every idea $\langle\mathrm{A}\rangle$ tends to be neutralized, diminished, balanced by $<$ Non-A $>$ ideas (not only $<$ Anti-A $>$ as Hegel asserted) - as a state of equilibrium. In between $\langle\mathrm{A}\rangle$ and $<$ Anti-A $>$ there are infinitely many $<$ Neut-A $>$ ideas, which may balance $<\mathrm{A}>$ without necessarily $<$ Anti-A $>$ versions.

To neuter an idea one must discover all its three sides: of sense (truth), of nonsense (falsity), and of undecidability (indeterminacy) - then reverse/combine them. Afterwards, the idea will be classified as neutrality.

\section{K)Delimitation from Other Philosophical Concepts and Theories:}

1. Neutrosophy is based not only on analysis of oppositional propositions, as dialectic does, but on analysis of neutralities in between them as well.

2. While epistemology studies the limits of knowledge and justification, neutrosophy passes these limits and takes under magnifying glass not only defining features and substantive conditions of an entity $\langle\mathrm{E}\rangle$ - but the whole $<\mathrm{E}^{\prime}>$ derivative spectrum in connection with $<$ Neut-E $>$.

Epistemology studies philosophical contraries, e.g. $<$ E $>$ versus $<$ Anti-E $>$, neutrosophy studies $<$ Neut-E $>$ versus $<\mathrm{E}>$ and versus $<$ Anti-E $>$ which means logic based on neutralities.

3-4. Neutral monism asserts that ultimate reality is neither physical nor mental. Neutrosophy considers a more than pluralistic viewpoint: infinitely many separate and ultimate substances making up the world. 
5. Hermeneutics is the art or science of interpretation, while neutrosophy also creates new ideas and analyzes a wide range ideational field by balancing instable systems and unbalancing stable systems.

6. Philosophia Perennis tells the common truth of contradictory viewpoints, neutrosophy combines with the truth of neutral ones as well.

7. Fallibilism attributes uncertainty to every class of beliefs or propositions, while neutrosophy accepts $100 \%$ true assertions, and 100\% false assertions as well - moreover, checks in what referential systems the percent of uncertainty approaches zero or 100.

\section{L) Philosophy's Limits:}

The whole philosophy is a tautologism: true in virtue of form, because any idea when first launched is proved true by its initiator(s). Therefore, philosophy is empty or uninformative, and a priori knowledge.

One can ejaculate: All is true, even the false!

And yet, the whole philosophy is a nihilism: because any idea, first proved true, is later proved false by followers. It is a contradiction: false in virtue of form. Therefore, now philosophy is overinformative, and a posteriori knowledge.

Now, one can ejaculate: All is false, even the truth!

All not-yet-contradicted philosophical ideas will be sooner or later contradicted because every philosopher attempts to find a breach in the old systems. Even this new theory (that I am sure it is not pretty sure!) will be inverted... And, later, others will reinstall it back...

Consequently, philosophy is logically necessary and logically impossible. Agostino Steuco of Gubbio was right, the differences between philosophers are undifferentiable. Leibniz's expression <true in all possible world> is superfluous, derogatory, for our mind may construct impossible world as well, which become possible in our 
imagination (F.Smarandache, "Inconsistent Systems of Axioms", 1995).

- In this theory one can prove anything!

- In this theory one can deny anything!

Philosophism $=$ Tautologism + Nihilism.

\section{M) Classification of Ideas:}

a) Easily accepted, quickly forgotten;

b) Easily accepted, heavily forgotten;

c) Heavily accepted, quickly forgotten;

d) Heavily accepted, heavily forgotten.

And various versions in between any two categories. ${ }^{2}$

\section{N) Evolution of an Idea:}

$<\mathrm{A}>$ in the world is not cyclic (as Marx said), but discontinuous, knotted, boundless:

$<$ Neut-A $>=$ existing ideational background, before arising $<\mathrm{A}>$;

$<$ Pre-A $>=$ a pre-idea, a forerunner of $<$ A $>$;

$<$ Pre-A' $>=$ spectrum of $<$ Pre-A $>$ versions;

$<\mathrm{A}>=$ the idea itself, which implicitly gives birth to

$<$ Non- $\mathrm{A}\rangle=$ what is outer $\langle\mathrm{A}\rangle$;

$\left\langle\mathrm{A}^{\prime}\right\rangle=$ spectrum of $\langle\mathrm{A}\rangle$ versions after (miss) interpretations (miss) understanding by different people, schools, cultures;

$<\mathrm{A} /$ Neut-A $>=$ spectrum of $\langle\mathrm{A}>$ derivatives/deviations, because $<A>$ partially mixes/melts first with neuter ideas;

$<$ Anti-A $>=$ the straight opposite of $<A>$, developed inside of $<$ Non-A $>$;

$<$ Anti-A' $>$ spectrum of $<$ Anti-A $>$ versions after (miss) interpretations (miss) understanding by different people, schools, cultures;

$<$ Anti-A/Neut-A $>=$ spectrum of $<$ Anti-A $>$ derivatives/ deviations, which means partial $<$ Anti-A $>$ and partial $<$ Neut-A $>$ combined in various percentage;

$<\mathrm{A}^{\prime} /$ Anti- $\left.^{\prime}\right\rangle=$ spectrum of derivatives/deviations after mixing $\left\langle\mathrm{A}^{\prime}>\right.$ and $<$ Anti- $\mathrm{A}^{\prime}>$ spectra; 
$<$ Post-A $>=$ after $<$ A $>$, a post-idea, a conclusiveness;

$<$ Post-A' $>=$ spectrum of $<$ Post-A $>$ versions;

$<$ Neo-A $>=<\mathrm{A}>$ retaken in a new way, at a different level, in new conditions, as in a non-regular curve with inflection points, in evolute and involute periods, in a recurrent mode; the life of $<\mathrm{A}>$ restarts.

Marx's 'spiral' of evolution is replaced by a more complex differential curve with ups-and-downs, with knots - because evolution means cycles of involution too.

This is dynaphilosophy = the study of infinite road of an idea.

$<$ Neo-A $>$ has a larger sphere (including, besides parts of old $<\mathrm{A}>$, parts of $<$ Neut-A $>$ resulted from previous combinations), more characteristics, is more heterogeneous (after combinations with various $<$ Non-A $>$ ideas). But, $<$ Neo-A $>$, as a whole in itself, has the tendency to homogenize its content, and then to de-homogenize by mixture with other ideas.

And so on, until the previous $<\mathrm{A}>$ gets to a point where it paradoxically incorporates the entire $<$ Non-A $>$, being indistinct of the whole. And this is the point where the idea dies, can not be distinguished from others. The Whole breaks down, because the motion is characteristic to it, in a plurality of new ideas (some of them containing grains of the original $<\mathrm{A}>$ ), which begin their life in a similar way. As a multinational empire, it is not possible to pass from an idea to its opposite without crossing over a spectrum of idea's versions, deviations, or neutral ideas in between.

Thus, in time, $<\mathrm{A}>$ gets to mix with $<$ Neut-A $>$ and $<$ Anti-A $>$. We wouldn't say that "extremes attract each other", but $<\mathrm{A}>$ and $<$ Non-A $>$ (i.e., inner, outer, and neutron of an idea).

Therefore, Hegel was incomplete when he resumed that: a thesis is replaced by another, called anti-thesis; contradiction between thesis and anti-thesis is surpassed and thus solved by 
a synthesis. So Socrates in the beginning, or Marx and Engels (dialectic materialism). There is not a triadic scheme:

- thesis, antithesis, synthesis (Hegelians);

Or

- assertion, negation, negation of negation (Marxists);

but a pluradic pyramidal scheme, as seen above.

Hegel's and Marx's antithesis <Anti-T> does not simply arise from thesis $<\mathrm{T}>$ only. $<\mathrm{T}>$ appears on a background of preexistent ideas, and mixes with them in its evolution. $<$ Anti-T $>$ is built on a similar ideational background, not on an empty field, and uses in its construction not only opposite elements to $\langle\mathrm{T}>$, but elements of $<$ Neut-T $>$ as well, and even elements of $<\mathrm{T}>$.

For, a thesis $\langle\mathrm{T}>$ is replaced not only by an antithesis $<$ Anti-T $>$, but also by various versions of neutralities $<$ Neut-T $>$.

We would resume this at: neuter-thesis (ideational background before thesis), pre-thesis, thesis, pro-thesis, non-thesis (different, but not opposite), anti-thesis, post-thesis, neo-thesis.

Hegel's scheme was purist, theoretic, idealistic. It had to be generalized. From simplism to organicism.

\section{O)Philosophical Formulas:}

Why are there so many distinct (even contrary) philosophical Schools? Why, concomitantly with the introduction of a notion $<\mathrm{A}\rangle$, its reverse $<$ Non- $\mathrm{A}\rangle$ is resulting?

Now, one presents philosophical formulas just because in the spiritual field it is really difficult to obtain (exact) formulas.

\section{a) Law of Equilibrium:}

The more $<\mathrm{A}>$ increases, the more $<$ Anti-A $>$ decreases. One has the following relationship: 


$$
<\text { A }>\cdot<\text { Anti-A }>=\mathrm{k} \cdot<\text { Neut-A }>\text {, }
$$

where $\mathrm{k}$ is a constant depending on $\langle\mathrm{A}>$, and $<$ Neut- $\mathrm{A}>$ is a supporting point for balancing the two extremes. If the supporting point is the neutralities' centroid, then the above formula is simplified to:

$$
<\text { A }>\cdot<\text { Anti-A }>=\mathrm{k}
$$

Where $\mathrm{k}$ is a constant depending on $<\mathrm{A}>$

Interesting particular cases:

- Industrialization $\times$ Spiritualization $=$ constant, for any society.

The more industrialized a society is, the less spiritual level its citizens have.

- Science $\times$ Religion $=$ constant. $^{3}$

- White $\times$ Black $=$ constant.

- Plus $\times$ Minus $=$ constant.

Pushing to the limits, in other words calculating in the absolute space, one gets:

Everything $\times$ Nothing $=$ universal constant,

Or $\infty \times 0(=0 \times \infty)=$ universal constant.

We are directing towards a mathematization of philosophy, but not in a Platonian sense.

Graph 5. O.a.1:

Materialism $\times$ Idealism $=$ constant, for any society.

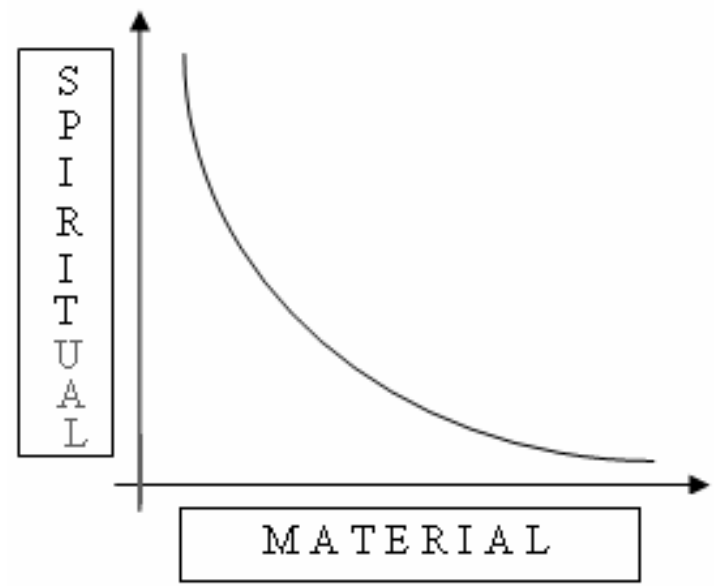


The vertical and horizontal Cartesian axes are asymptotes for the curve $\mathrm{M} \cdot \mathrm{I}=\mathrm{k}$.

\section{b) Law of Anti-Reflexivity:}

$<A>$ in the mirror of $\angle A>$ gradually vanishes itself.

Or $<A>$ of $<A>$ may transform into a distorted $<A>$.

Examples:

- Marriage between relatives gives birth to vapid (often handicapped) descendants.

That's why crossing the species of plants (and sometimes races of animals and humans as well) we get hybrids with better qualities and/or quantities. Biological theory of mixing species.

That's why emigration is benign for bringing new blood in a static population.

- Nihilism, spread out after Turgeniev's "Parents and children" novel in 1862 as an absolute negation, denies everything, therefore itself too!

- Dadaism of the dadaism vanishes either.

\section{c) Law of Complementarity:}

$<$ A $>$ feels like completing with $<$ Non-A $>$ in order to form a whole.

Examples:

- Persons, who are different, feel like completing each other and associate. (Man with woman.)

- Complementary colors (that, combined in the right intensities, produce white).

\section{d) Law of Inverse Effect:}

When trying to convert someone to an idea, belief, or faith by boring repetitions or by force, he ends up to hating it. ${ }^{4}$

Examples:

- The more you ask someone to do something, the less he would. 
- Doubling the rule, brings to halving.

- What's much, it's not good... (inversely proportional). ${ }^{5}$

- When you are sure, don't be!

- When pressing someone to do something, he would do a different (but not necessary the opposite, as Newton's third motion laws' axiom stated ${ }^{6}$ ) at-various-slopes reaction:

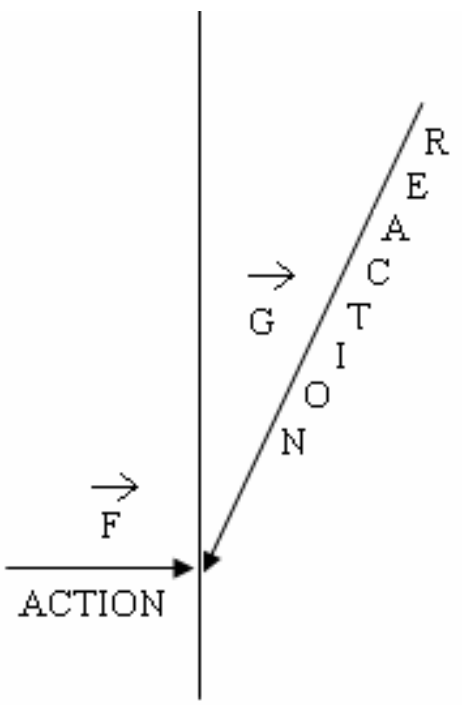

\section{e) Law of Reverse Identification:}

$<$ Non- $\mathrm{A}>$ is a better $<\mathrm{A}>$ than $<\mathrm{A}>$

Example:

Poetry is more philosophical than philosophy.

\section{f) Law of Joined Disjointedness:}

$<\mathrm{A}>$ and $<$ Non- $\mathrm{A}>$ have elements in common

Examples:

- There is little distinction between "good" and "bad".

- Rational and irrational work together unseparately.

- Consciousness and unconsciousness similarly. 
- "Come, my soul said, let's write poems for my body, for we are One" (Walt Whitman).

- Finite is infinite [see the microinfinity].

\section{g) Law of Identities' Disjointedness:}

The permanent fight between $\left\langle\mathrm{A}>\right.$ and $\left\langle\mathrm{A}^{\prime}\right\rangle$ (different shades of $<\mathrm{A}>$ ).

Examples:

- The permanent fight between absolute truth and relative truth.

- The distinction between crisp false and neutrosophic false (the second one means a combination of falsity, indeterminacy, and truth degrees).

\section{h) Law of Compensation:}

$$
\text { If }<\mathrm{A}>\text { now, then }<\text { Non-A }>\text { later }
$$

Examples:

- Any loss / Has its gain [meaning later it will be better, because you learned from the loss].

- There is no success without failure [patience guys!]. ${ }^{7}$

\section{i) Law of Prescribed Condition:}

One cannot jump out of own limits.

(One spins inside own circle.)

\section{j) Law of Particular Ideational Gravitation:}

Every idea $<\mathrm{A}>$ attracts and rejects other idea $<\mathrm{B}>$ with a force directly proportional with the product of their neutrosophic measures and the exponential of their distance. (By opposition to the modern restatement of Newton's law of gravitation of particles of matter, the distance influences directly - not indirectly - proportional: the more opposite (distanced) ideas, the stronger attraction) 


\section{k) Law of Universal Ideational Gravitation:}

$<\mathrm{A}>$ tends towards $<$ Non-A $>$ (not $<$ Anti-A $>$ as Hegel said), and reciprocally. There are forces which act on $<A>$, directing it towards $<$ Non- $A>$, until a critical point is attained, and then $\langle\mathrm{A}\rangle$ turns back. $\langle\mathrm{A}\rangle$ and $\langle$ Non- $\mathrm{A}\rangle$ are in a continuous motion, and their frontiers changing accordingly.

Examples:

- Perfection leads to imperfection. ${ }^{8}$

- Ignorance is pleased.

Particular Case:

Everybody tends to approach his specific level of incompetence!

This is not a joke, but very truly:

$\mathrm{X}$ gets a job at level say L1; if he is good, he's promoted to level L2; if, in the new position he's good, he's promoted further to L3; and so on... until he's not good anymore, therefore not promoted; thus, he got to his level of incompetence.

$<\mathrm{A}>$ tends towards $<$ Non-A $>$

Therefore, everybody's ideal is to tend towards what he/she is not able to do.

But the movement is nonlinear;

$<$ Non-A $>$ has a large range (power of continuum) of "what is not $\angle \mathrm{A}>$ " (outer $\angle \mathrm{A}>$ ) versions, let index them in the set $\left.\{<\text { Non-A }\rangle_{i}\right\}_{i}$. (All $\left\{<\text { Anti-A }>_{i}\right\}_{i}$ versions are included in $<$ Non-A $\rangle_{\text {. }}$ Hence, infinitely many $<$ Non- $\left.A\right\rangle_{i}$ versions gravitate, as planets around a star, on orbits of $\langle\mathrm{A}\rangle$. And, between each $\langle\text { Non- } \mathrm{A}\rangle_{\mathrm{i}}$ version and the centroid "star" $\langle\mathrm{A}\rangle$, there are attraction and rejection forces. They approach each other until arriving to certain minimum critical points: $\mathrm{P}_{\mathrm{m}(\mathrm{i})}$ for $<\mathrm{A}>$, and $\mathrm{Q}_{\mathrm{m}(\mathrm{i})}$ for $<$ Non- $\mathrm{A}>_{\mathrm{i}}$, and then again they go far from each other until touching certain maximum points:

$\mathrm{P}_{\mathrm{M}(\mathrm{i})}$ for $<\mathrm{A}>$, and $\mathrm{Q}_{\mathrm{M}(\mathrm{i})}$ for $<$ Non- $\mathrm{A}>_{\mathrm{i}}$

Through differential equations we may calculate the minimum and maximum (spiritual) distances between $<\mathrm{A}>$ and $<$ Non- $A\rangle_{\text {i }}$, the Cartesian coordinates of the critical points, 
and the status quo of each version. We would say that $\langle\mathrm{A}\rangle$ and a $<$ Non- $\mathrm{A}>_{\mathrm{i}}$ version meet in an absolute/infinite point. When all $\langle\text { Non- } \mathrm{A}\rangle_{\mathrm{i}}$ versions fall into $\langle\mathrm{A}\rangle$ we have a catastrophe!

\subsection{Neutrosophic Epistemology}

This section, which is a neutrosophic epistemology, has a structure alike Wittgenstein's tractatus: short (from 1-2 lines to maximum 10-15 lines) independent philosophical reflections, metaphysical and metaphorical comments which are separated by blank rows. It is an analytical study, and it is related to multiple-valued logic because in almost each small paragraph one shows that a statement $<\mathrm{A}>$ was proved true by a philosopher $\mathrm{X}$ whereas latter another philosopher $\mathrm{Y}$ proved the opposite statement $<$ Anti-A $>$ was true. Therefore, both $<\mathrm{A}>$ and $<$ Anti-A $>$ were true. \{Whence one can deduce that both $\angle \mathrm{A}>$ and $<$ Anti-A $>$ could be false. $\}$ Even more, using a neutrosophic interpretation, one could say that other ideas in between $<\mathrm{A}>$ and $<$ Anti-A $>$ and related to them, noted by $<$ Neut-A $>$, could be true as well. This relates to dialetheism, which says that some contradictions are true, to paraconsistent logic, to intuitionistic logic, till neutrosophic logic (where $<\mathrm{A}>$, $<$ Anti-A $>$, and ideas in between them belonging to $<$ Neut-A $>$ could all be true or partially true).

Many paradoxes are treated here, and one knows that a paradox is a proposition true and false in the same time - i.e. connected to multiple-valued logic as well, and not many logics approached the paradoxes. Other reflections show that a subject may be characterized by an attribute and its opposite simultaneously (also related to multiple-valued logic in a philosophical way).

- To any launched idea there are pro- and contra- reactions, but also neuter (indifference, neutrality) as well. Hegel's $<$ dialectic $>$ [Gr. dialektikĕ $<$ dia with, legein to speak] 
doesn't work, it consequently has to be extended to a somehow improper term trialectic, and even more to a pluralectic because there are various degrees of positive, and of negative, and of indifference as well - all of them interpenetrated. Going to a continuum-power transalectic ( $\infty$-alectic).

- " "+" not only asks for "-" for equilibrium, as Hegel said, but for " 0 " as well as a support point for the thinking lever. Hegel's self-development of an idea $\langle A>$ is not determined on its internal contraries only, but on its neutralities as well - because they all fare and interfere. Self-development of an idea is also determined by external (pro, contra, neuter) factors (Comparative Philosophy, as comparative literature).

- Between particular and universal there are $\mathrm{P} \%$ particular, $\mathrm{I} \%$ indeterminate (neutral), and $\mathrm{U} \%$ universal things, with $\mathrm{P}, \mathrm{I}, \mathrm{U} \subset]^{-0} 0,1^{+}[$.

- The atom's structure holds in the history of any idea. The reasoning is based upon the analysis of positive, negative, and neutral propositions.

This should be called Quantum Philosophy.

- In nuclear fission a free neutron strongly interacts with nuclei and is readily absorbed, then it decays into a proton, an electron, and a 'neutrino' (Enrico Fermi) with a half-time of near 12 minutes.

- Neutrosophy studies not only an idea's conditions of possibility, but of impossibility as well. And focuses on its historical development (past and present interpretation - by using classical analysis, and future interpretation - by using neutrosophic probability and statistics).

- In economics Keynes chose for the concept of "unstable equilibrium" (<The General Theory $>$ ), whereas Anghel M. Ruginã passed to that of "stable disequilibrium" ( $<$ Truth in the Abstract (Analytical) versus Truth in the Concrete (Empirical) $>$ ). A self-regulating and self-unregulating 
mechanism is functioning in each system, moving from equilibrium to dis-equilibrium back and forth. A unstablemade stability, and stable-made instability. Or equilibrium in disequilibrium, and disequilibrium in equilibrium.

- We mean, a very dynamic system by rapid small changes, characterized by a derivative. The static system is dead. Leon Walras was right: monopolies reduce the competitions, and thus the progress.

- My opinion is that some philosophers grope, stumble. They don't have clear ideas or systems, or even precise directions on a subject. Paroles, paroles... What one asserts today, another will deny tomorrow. Many times they talk too much for saying nothing. Some have points contrary to experience and evidence, others have an inadequate reason.

That is why a mathematization (even more, an axiomatization but not in stricto sensu) of all knowledge fields would be required, especially in philosophy (alike Mendeleev's Table of Chemical Elements).

The mathematization is required because it is not possible!

- Philosophy is semiscientific and semiempiric. It is less scientific than psychology, but more scientific than poetry.

- Human is dependent and independent in the same time.

- I understand spirit as quality, and material as quantity. Of course, they melt.

- I see truth like a body, an object with a form.

I see material as a dense/condensed spirit, a viscous idea.

- The structure of ideas reflects the structure of objects. And reciprocally.

- In the mind-body problem:

The mental phenomenon is of physical nature, and the physical phenomenon of mental as well.

\section{- Neohegelians:}

Reconciliation of contraries (Bradley), or irreconciliation of contraries (Wahl)? Both! 


\section{- Neutrosophy.}

- has the aim of unifying field in humanistic (as Einstein tried to find in science);

- explores the differences between:

- thinkers,

- philosophical schools, movements, theories, principles and proves they are minimum;

- reveals that no thought school is better than another, and no philosopher is greater than another;

- is an attempt to reconcile reluctant viewpoints with inoffensive others;

- the truth may not be separated from false;

- if the philosopher $\mathrm{X}$ enunciated a proposition $\mathrm{P}$, try to contrarily think and to compare with $<$ Neut-P $>$ too.

\section{- Ignorantism:}

- Power countries deliberately ignore the arts, literature, science, culture, traditions of third world countries. More, they even boycott, scorn them...

- Third world countries creators and inventors are also handicapped by language, poor living conditions, less technology for doing research.

- In histories of arts, literature, science you see only westerners.

- rare exceptions of other people being in there confirm the rule!

- A minor poet, for example, who wrote in English or French or German, is better known than a genius like Eminescu who wrote in a not-international Romanian language.

- Negativity (Heraclitus, Spinoza, Kant, and Hegel) passes through diverse phases: from assertion to a spectrum of partial negativity, and eventually to a higher degree negativity.

- Not com-plementarity (used by Bohr and Heisenberg in physics philosophy), but tri-plementarity (negat- ivity, 
positivity, and neutrality - corresponding to 0,1 , and $1 / 2$ respectively), even $n$-plementarity (which means: $n$ disjoint elements together forming a whole), or generalized to $\infty$ plementarity (with power of continuum), for there are complex mixed versions of them.

Beyond indefinitely many states between 0 and 1 , the midpoint $1 / 2$ represents neither negative, nor positive - or represents both of them (which cancel each other).

- Hermeneutics of Philosophical Hermeneutics:

If prejudgments can not be eliminated in the judgements, why do we need the science of interpretation?.

- Arguing with Plekhanov (historical development is not managed at will), one says that it is at some degree managed and at another degree not managed by the will.

- Ab'lard's conceptualism, which states that universalia post rem (general is besides things), i.e. general is not in things, is partially true, because general persists in each individual, that is why it is possible to form classes of individuals with similar particular characteristics.

\section{- Philosophy of Philosophy.}

- why do we need philosophy today?

- why don't we need philosophy today?

- what direction is philosophy going to?

- what direction isn't philosophy going to?

One feels that philosophy is for people who have nothing else to do, like puzzles or rebus!

\section{- Neutrosophy means/encompasses:}

- philosophy seen by a mathematician and poet;

- study of History of Philosophy;

- controversial themes of philosophy (to explore the offensiveness and inoffensiveness);

- evolution of an idea from $<\mathrm{A}>$ to $<$ Non- $\mathrm{A}>$ and then to $<$ Anti-A $>$; 
- how to get patterns where they do not look to be, i.e. to find common characteristics at "+", "-", and "0" attributes;

- how an idea appears from different viewpoints, from all viewpoints;

- to find the vanishing point of all philosophical ideas.

\section{- Neutrosophy can also be seen as:}

- new approach to philosophy;

- philosophy of philosophies;

- non-philosophy;

- super-philosophy;

- neophilosophy;

- God and Devil of the philosophy;

- meta-philosophy, macro-philosophy;

- New World Order in philosophy;

- paradox of philosophy and philosophy of the paradox;

- thought of thought;

- showing the philosophy's perfection and imperfection simultaneously;

- paradox within/from paradox: there are infinitely many;

- world's enigma;

- nature's essence;

- enigma of the world;

- any substance ultimately has a neutrosophic attribute;

- life without paradox would be monotonous and boring, linear;

- paradoxist intuition is a high level of awareness;

- postmodernist;

- an algebraic, physical and chemical philosophy;

- consistent with its inconsistence.

- Transcendentalism (Emerson especially, and Kant, Hegel, Fichte), which proposes to discover the nature of reality by investigating the process of thought, is combined with 
pragmatism (Williams James), which first "tries to interpret each notion or theory by tracing its respective practical consequences".

We mean to know reality through thought, and thought through reality.

- In India's VIII-th - IX-th centuries one promulgated the Non-Duality (Advaita) through the non-differentiation between Individual Being (Atman) and Supreme Being (Brahman). The philosopher Sańkaracharya (782-814 A.C.) was then considered the savior of Hinduism, just in the moment when the Buddhism and the Jainism were in a severe turmoil and India was in a spiritual crisis.

Non-Duality means elimination of ego, in order to blend yourself with the Supreme Being (to reach the happiness). Or, arriving to the Supreme was done by Prayer (Bhakti) or Cognition (Jnana). It is a part of Sańkaracharya's huge merit (charya means teacher) the originality of interpreting and synthesizing the Source of Cognition (Vedas, IV th century B.C.), the Epic (with many stories), and the Upanishads (principles of Hindu philosophy) concluding in Non-Duality.

Then Special Duality (Visishta Advaita) follows, which asserts that Individual Being and Supreme Being are different in the beginning, but end to blend themselves (Rāmānujacharya, XI-th century).

And later, to see that the neutrosophic scheme perfectly functions, Duality (Dvaita) ensues, through whom the Individual Being and Supreme Being were differentiated (Madhvacharya, XIII - th- XIV-th centuries).

Thus: Non-Duality converged to Duality:

$<$ Non-A $>$ converges to $<\mathrm{A}>$

- Know yourself to know the others.

Study the others to understand yourself.

- In conclusion, I want to be what I don't want to be:

a Philosopher. That's why I am not.

(That's why, maybe, am I?) 
- Control what you can, leave the rest to the luck. Control what you cannot, free what you control.

\section{- Contradictory Theory:}

Why do people avoid thinking about a contradictory theory?

- As you know, nature is not perfect:

and opposite phenomena occur together, and opposite ideas are simultaneously asserted and, ironically, proved that both of them are true! How is that possible? ...

A statement may be true in a referential system, but false in another one. The truth is subjective. The proof is relative.

(In philosophy there is a theory: that "knowledge is relative to the mind, or things can be known only through their effects on the mind, and consequently there can be no knowledge of reality as it is in itself"), called "the Relativity of Knowledge"; <Webster's New World Dictionary of American English>, Third College Edition, Cleveland \& New York, Simon \& Schuster Inc., Editors: Victoria Neufeldt, David B. Guralnik, p. 1133, 1988).

You know?... Sometimes is good to be wrong!

- Continuum Hypothesis (that the cardinality of the continuum is the smallest non-denumerable cardinal) has been shown to be undecidable, in that both it and its negation are consistent with the standard axioms of set theory.

- By contrast to the relativism, which asserts that there is no absolute knowledge, in neutrosophy it is possible to attaint in pure science and by convention the absolute truth, $\mathrm{t}=100$, and yet as a matter of rare fact.

\section{- Hermeneutics of Hermeneutics:}

An idea $<\mathrm{A}>$, by interpretation, is generalized, is particularized, is commented, is filtered, eventually distorted to $<\mathrm{A} 1>$ different from $<\mathrm{A}>$, to $<\mathrm{A} 2>$ different from $<\mathrm{A}>$, and so on. 
- Everybody understands what he wants, according to his level of knowledge, his soul, and his interest.

$<\mathrm{A}>$ is viewed as $\langle$ Non- $\mathrm{A}>$ and even $<\mathrm{Anti}-\mathrm{A}\rangle$ at some degree (ill-defined). But all deformed versions of this idea syncretize in an $\angle A>$ way.

- Idealists were so formal, empiricists so informal. Neutrosophy is both.

\section{- Sociological Theory:}

As in the Primitive Society, the modern society is making for MATRIARCHATE - the woman leads in the industrialized societies. From an authoritarian PATRIARCHATE in the Slavery and Feudalism towards a more democratic MATRIARCHATE at present.The sexuality plays an immense role in the manipulation of men by women, because the women "have monopoly of the sex" as was justifying to me an American friend kept by his wife henpecked!

A cyclic social development.

The woman becomes the center of the society's cell, the family.The sexual pleasure influences different life circles, from the low class people to the leading spheres. Freud was right...

One uses women in espionage, in influencing politicians' decisions, in attracting businessmen - by their feminine charms, which obtain faster results than their male proponents. The women have more rights than men in western societies (in divorce trials).

\section{- Social Paradox:}

In a democracy should the nondemocratic ideas be allowed?

a) If no, i.e. other ideas are not allowed - even those nondemocratic -, then one not has a democracy, because the freedom of speech is restricted.

b) If yes, i.e. the nondemocratic ideas are allowed, then one ends up to a nondemocracy (because the non- 
democratic ideas overthrow the democracy as, for example, it happened in Nazi Germany, in totalitarian countries, etc.).

\section{- The Sets' Paradox:}

The notion of "set of all sets", introduced by Georg Cantor, does not exist. Let all sets be noted by $\left\{\mathrm{S}_{\mathrm{a}}\right\}_{\mathrm{a}}$, where a indexes them. But the set of all sets is itself another set, say $\mathrm{T}_{1}$; and then one constructs again another "set of $<$ all sets $>$ ", but $<$ all sets $>$ are this time $\left\{\mathrm{S}_{\mathrm{a}}\right\}$ and $\mathrm{T}_{1}$, and then the "set of all sets" is now $\mathrm{T}_{2}$, different from $\mathrm{T}_{1}$; and so on... .

Even the notion of "all sets" can not exactly be defined (like the largest number of an open interval, which doesn't exist), as one was just seeing above (we can construct a new set as the "set of all sets") and reunites it to "all sets".

- A Paradoxist Psychological Complex (with the accent on the first syllable):

A collection of fears stemming from previous unsuccessful experience or from unconscious feelings that, wanting to do something $<\mathrm{S}>$, the result would be $<$ Anti$\mathrm{S}>$, which give rise to feelings, attitudes, and ideas pushing the subject towards a deviation of action $<\mathrm{S}>$ eventually towards an $<$ Anti-S $>$ action.

(From the positive and negative brain's electrical activities.)

How to manage this phobia? To dote and anti-dote! By transforming it into an opposite one, thinking differently, and being fear in our mind that we would pass our expectancies but we shouldn't.

People who do not try of fear not to be rejected: they lose by not competing!

\section{- Auto-suggestion:}

If an army leaves for war with anxiety to lose, that army is half-defeated before starting the confrontation.

\section{- Paradoxist Psychological Behavior:}

How can we explain contrary behaviors of a person: in the same conditions, without any reason, cause? 
Because our deep unconsciousness is formed of contraries.

- Ceaseless Anxiety:

What you want is, normally, what you don't get. And this is for eternity. Like a chain...

Because, when you get it (if ever), something else will be your next desire. Man can't live without a new hope.

\section{- Inverse Desire:}

The wish to purposely have bad luck, to suffer, to be pessimistic as stimulating factors for more and better creation or work. (Applies to some artists, poets, painters, sculptors, spiritualists.)

\section{- My Syndrome:}

Is characterized by nose frequently bleeding under stress, fear, restlessness, tiredness, nervousness, prolo- nged unhappiness. This is the way the organism discharges, thus re-establishing the equilibrium, and it is fortunate because the hemorrhage is not interior which would cause death of patient.

The bleeding is cause by the nervous system, not by physical injury. If you have any idea of treating it, don't hesitate to contact the author. All opinions are welcome.

- All is possible, the impossible too!

Is this an optimistic or pessimistic paradox?

a) It is an optimistic paradox, because shows that all is possible.

b) It is a pessimistic paradox, because shows that the impossible is possible.

\section{- Mathematician's Paradox:}

Let $\mathrm{M}$ be a mathematician who may not be characterized by his mathematical work.

a) To be a mathematician, $\mathrm{M}$ should have some mathematical work done; therefore, $\mathrm{M}$ should be characterized by that work.

b) The reverse judgment: if $M$ may not be characterized by his mathematical work, then $\mathrm{M}$ is not a mathematician. 


\section{- Expect the Unexpected:}

If we expect someone to do the unexpected, then:

- Is it possible for him to do the unexpected?

- Is it possible for him to do the expected?

If he does the unexpected, then that's what we expected.

If he doesn't do the expected, then he did the unexpected.

\section{- The Ultimate Paradox:}

Living is the process of dying.

Reciprocally: Death of one is the process of some- body else's life [an animal eating another one].

- Exercises for readers:

- If China and Japan are in the Far East, why from USA do we go west to get there?

- Are humans inhuman, because they committed genocides?

\section{- The Invisible Paradoxes:}

- Our visible world is composed of a totality of invisible particles.

- Things with mass result from atoms with quasi-null mass.

- Infinity is formed of finite part(icle)s.

- Look at these Sorites Paradoxes (associated with Eubulides of Miletus (fourth century B.C.) :

a) An invisible particle does not form a visible object, nor do two invisible particles, three invisible particles, etc.

However, at some point, the collection of invisible particles becomes large enough to form a visible object, but there is apparently no definite point where this occurs.

b) A similar paradox is developed in an opposite direction.

It is always possible to remove an atom from an object in such a way that what is left is still a visible 
object. However, repeating and repeating this process, at some point, the visible object is decomposed so that the left part becomes invisible, but there is no definite point where this occurs.

Between $<\mathrm{A}>$ and $<$ Non-A $>$ there is no clear distinction, no exact frontier. Where does $<\mathrm{A}>$ really end and $<$ Non-A $>$ begin? We extend Zadeh's fuzzy set term to fuzzy concept.

- Uncertainty Paradox: Large matter, which is under the 'determinist principle', is formed by a totality of elementary particles, which are under Heisenberg's ' indeterminacy principle'.

- Unstable Paradox: Stable matter is formed by unstable elementary particles (elementary particles decay when free).

- Short Time Living Paradox: Long time living matter is formed by very short time living elementary particles.

- Paradoxist Existentialism: life's value consists in its lack of value ; life's sense consists in its lack of sense.

- Semantic Paradox (I): I AM WHO I AM NOT.

- If I am not Socrates, and since I am who I am not, it results that I am Socrates.

- If I am Socrates, and since I am who I am not, it results that I am not Socrates.

Generally speaking: "I am X", if and only if "I am not X". Who am I?

In a similar pattern one constructs the paradoxes:

I AM MYSELF WHEN I AM NOT MYSELF.

I EXIST WHEN I DON'T EXIST.

And, for the most part:

I $\{$ verb $\}$ WHEN I DON'T $\{$ verb $\}$.

(F. Smarandache, "Linguistic Paradoxes")

- Semantic Paradox (II): I DON'T THINK.

This can not be true for, in order to even write this sentence, I needed to think (otherwise I was writing with 
mistakes, or was not writing it at all). Whence "I don't think" is false, which means "I think".

\section{- Unsolved Mysteries:}

a) Is it true that for each question there is at least an answer?

b) Is any statement the result of a question?

c) Let $\mathrm{P}(\mathrm{n})$ be the following assertion:

"If $\mathrm{S}(\mathrm{n})$ is true, then $\mathrm{S}(\mathrm{n}+1)$ is false", where $\mathrm{S}(\mathrm{n})$ is a sentence relating on parameter $n$.

Can we prove by mathematical induction that $\mathrm{P}(\mathrm{n})$ is true?

d) $"<\mathrm{A}>$ is true if and only if $<\mathrm{A}>$ is false".

Is this true or false?

e) How can this assertion "Living without living" be true?

Find a context. Explain.

- $<$ Anti-A $>$ of $<$ A $>$

Anti-literature of literature

$$
<\text { Non-A }>\text { of }<\text { A }>
$$

Language of non-language

$<$ A $>$ of $<$ Non- $A>$

Artistic of the non-artistic

- Tautologies:

- I want because I want. (showing will, ambition)

$<\mathrm{A}>$ because of $<\mathrm{A}>$

(F. Smarandache, "Linguistic Tautologies")

- Our axiom is to break down all axioms.

- Be patient without patience.

- The non-existence exists.

The culture exists through its non-existence.

Our culture is our lack of culture.

- Style without style.

- The rule we apply: it is no rule. ${ }^{9}$

- Paradox of the Paradoxes:

Is "This is a paradox" a paradox? 
I mean is it true or false?

- To speak without speaking. Without words (body language).

To communicate without communicating.

To do the un-do.

- To know nothing about everything, and everything about nothing.

- I do only what I can't!

If I can't do something, of course "I can do" is false.

And, if I can do, it's also false because I can do only what I am not able to do.

I cannot for I can

- Paradoxal sleep, from a French "Larousse" dictionary (1989), is a phase of the sleep when the dreams occur.

Sleep, sleep, but why paradoxal?

How do the dreams put up with reality?

- Corrupt the incorruptible!

- Facts exist in isolation from other facts (= the analytic philosophy), and in connection as well with each other (= Whitehead's and Bergson's thoughts).

The neutrosophic philosophy unifies contradictory and noncontradictory ideas in any human field.

- Platonism is the observable of unobservable, the thought of the non-thought.

- If vices wouldn't exist, the virtues will not be seen (T. Muşatescu). ${ }^{10}$

- Philosophy is a poetical science and a scientific poetry.

- There are three main types of humans: not only Nietzsche's "overman" with his will to power, but also the $<$ midman $>$ with his will to mediocrity (yes, people who love to anonymously live every single day, dull), and <underman> with his will to weakness (homeless, tramps, criminals who indulge in laziness, illegalities). 
Inside of every man there are an $<$ overman $>$, a $<$ midman $>$, and an <underman $>$ - varying in terms of moment, space, context.

That's why, generally speaking, every man is: $\mathrm{O} \%$ overman, $\mathrm{M} \%$ midman, and $\mathrm{U} \%$ underman, where:

$$
\mathrm{O}, \mathrm{M}, \mathrm{U} \subset]^{-0}, \mathrm{1}^{+}[
$$

- I see the ideas. They are red and blue and white, round and sharp, small and big and middle size. I look through the objects and see the essence.

- Our mind can not reflect truth accurately (Francis Bacon).

Unfortunately the science too.

What about arts? (No, they are too subjective.)

- And a sage: There is no philosophy, there are only philosophers. Therefore philosophers without philosophy!

But reciprocally: is there a philosophy without philosophers?

- Because any attempt to change the political power ends up in embarking another power, "the revolution is impossible " (Bernard-Henry Lévy, André Glucksmann, Jean-Marie Benoist, Philippe Némo who represented the "New Philosophy" French group).

- This is the Ultimate Idea: there is no ultimate idea!

- Schopenhaurer said "World is my idea" using $<$ vorstellung $>$ (Germ.) for <idea $>$, therefore material is immaterial (because 'idea' is 'immaterial').

- The politicians of the ancient world were always talking about morals and virtue, ours speak on nothing else but commerce and money (J. J. Rousseau).

- I AM NOT IGNORANT THAT I AM IGNORANT, parodying Socrates.

- Life is neutrosophic: crying today, laughing tomorrow, neither one after tomorrow...

- Mathematics also works with approximations. But exact approximations. 
And thus the perfection is a notion invented by human: an endeavor, a target never to be touched.

- We always want what we don't have. Once we've got it, our interest in it is lost. But we tend toward something else.

- Nietzsche: "All is chaos", but the chaos is organized, hair styled on the curlers of an uncombed head.

- Philosophy is useless. It is a headlache for individuals without head.

- Philosophers are inutile scientists. I am not a philosopher. Am I utile?

- If philosophy is inefficacious, let's do philosophy!

- The best philosophy is the total lack of philosophy? Because the non-philosophy is itself a philosophy.

- I didn't even want to become a philosopher in this mercantile society (for I would starve to death). That's why I philosophize... I try not to find a system.

- Today's people are very pragmatic; they don't give a penny on my neutrosophic arguments, nor on your anti-neutrosophic ones! Only for money they are caring...

- That's the crisis of the modern man's crisis!

Neutrosophic nature envelops everything.

- Jacques Derida's ideology: the death of all ideologies!

- Metaphysical sentences are neither true nor false, because they don't assert anything; they don't contain consciousness nor errors (Rudolf Carnap).

- It's easy to forget something important, but it's harder to forget something not important!

- Imaginary is more real than the reality.

- All is hatred, even the love.

- Knowledge is power (Francis Bacon), but knowledge brings weakness too (for example a cancerous who knows he's sick). 
Knowledge is power in science, research, but may be fear, suffering, even suicide - as in case of the previous patient, for example.

- Philosophy doesn't need philosophers, but thinkers. The thinkers don't need philosophy. Therefore, philos- ophy doesn't need philosophy!

- Remain with your real world - which exists, but I remain with my idealist world - which doesn't exist.

One exists through non-existence better.

- Man is a philosophical animal (but depraved, said Rousseau).

(Let's grade the degradation.)

- Philosophy is an alive graveyard of dead ideas.

- I write philosophy to denounce it, or to prove the sickness of philosophy (?)

- You don't need to be a philosopher in order to become a philosopher.

- I have decided not to decide anything anymore.

- "The beginning and ending are to be found on the same circle" (Heraclitus).

\subsection{Neutrosophic Logic}

\section{A) Why is the Neutrosophic Logic?}

As an alternative to the existing logics we propose the Neutrosophic Logic to represent a mathematical model of uncert- ainty, vagueness, ambiguity, imprecision, undefined, unknown, incompleteness, inconsistency, redundancy, contradiction. It is a non-classical logic.

Eksioglu (1999) explains some of them:

"Imprecision of the human systems is due to the imperfection of knowledge that human receives (observation) from the external world. Imperfection leads to a doubt about the value of a variable, a decision to be taken or a conclusion 
to be drawn for the actual system. The sources of uncertainty can be stochasticity (the case of intrinsic imperfection where a typical and single value does not exist), incomplete knowledge (ignorance of the totality, limited view on a system because of its complexity) or the acquisition errors (intrinsically imperfect observations, the quantitative errors in measures)."

"Probability (called sometimes the objective probability) process uncertainty of random type (stochastic) introduced by the chance. Uncertainty of the chance is clarified by the time or by events' occurrence. The probability is thus connected to the frequency of the events' occurrence."

"The vagueness which constitutes another form of uncertainty is the character of those with contours or limits lacking precision, clearness. [...] For certain objects, the fact to be in or out of a category is difficult to mention. Rather, it is possible to express a partial or gradual membership."

Indeterminacy means degrees of uncertainty, vagueness, imprecision, undefined, unknown, inconsistency, redundancy.

A question would be to try, if possible, to get an axiomatic system for the neutrosophic logic. Intuition is the base for any formalization, because the postulates and axioms derive from intuition.

\section{B) Definition:}

A logic in which each proposition is estimated to have the percentage of truth in a subset $\mathrm{T}$, the percentage of indeterminacy in a subset $\mathrm{I}$, and the percentage of falsity in a subset F, where T, I, F are defined above, is called Neutrosophic Logic.

We use a subset of truth (or indeterminacy, or falsity), instead of a number only, because in many cases we are not able to exactly determine the percentages of truth and of falsity but to approximate them: for example a proposition is between 30- 
$40 \%$ true and between $60-70 \%$ false, even worst: between 30 $40 \%$ or $45-50 \%$ true (according to various analyzers), and $60 \%$ or between $66-70 \%$ false.

The subsets are not necessary intervals, but any sets (discrete, continuous, open or closed or half-open/half-closed interval, intersections or unions of the previous sets, etc.) in accordance with the given proposition.

A subset may have one element only in special cases of this logic.

Statically T, I, F are subsets, but dynamically they are functions/operators depending on many known or unknown parameters.

\section{Constants:}

(T, I, F) truth-values, where T, I, F are standard or nonstandard subsets of the non-standard interval $]^{-} 0,1^{+}[$, where :

$$
\begin{gathered}
\mathrm{n}_{\text {inf }}=\inf \mathrm{T}+\inf \mathrm{I}+\inf \mathrm{F} \geq 0, \\
\text { And } \\
\mathrm{n}_{\text {sup }}=\operatorname{sup~} \mathrm{T}+\sup \mathrm{I}+\sup \mathrm{F} \leq 3^{+} .
\end{gathered}
$$

Statically T, I, F are subsets, but dynamically T, I, F are functions/operators depending on many known or unknown parameters.

Atomic formulas: $a, b, c .$.

Arbitrary formulas: A, B, C, ...

The neutrosophic logic is a formal frame trying to measure the truth, indeterminacy, and falsehood.

My hypothesis is that no theory is exempted from paradoxes, because of the language imprecision, metaphoric expression, various levels or meta-levels of understanding/ interpretation which might overlap.

\section{C) Differences between Neutrosophic Logic and} Intuitionistic Fuzzy Logic:

The differences between IFL and NL (and the corresponding intuitionistic fuzzy set and neutrosophic set) are:

a) Neutrosophic Logic can distinguish between absolute truth (truth in all possible worlds, according to Leibniz) and 
relative truth (truth in at least one world), because NL(absolute truth) $=1^{+}$while NL(relative truth) $=1$. This has application in philosophy (see the neutrosophy). That's why the unitary standard interval $[0,1]$ used in IFL has been extended to the unitary non-standard interval $]^{-} 0,1^{+}[$in NL.

Similar distinctions for absolute or relative falsehood, and absolute or relative indeterminacy are allowed in NL.

b) In NL there is no restriction on T, I, F other than they are subsets of $]^{-} 0,1^{+}[$, thus:

$-0 \leq \inf \mathrm{T}+\inf \mathrm{I}+\inf \mathrm{F} \leq \sup \mathrm{T}+\sup \mathrm{I}+\sup \mathrm{F} \leq 3^{+}$

This non-restriction allows paraconsistent, dialetheist, and incomplete information to be characterized in NL \{i.e. the sum of all three components if they are defined as points, or sum of superior limits of all three components if they are defined as subsets can be $>1$ (for paraconsistent information coming from different sources) or $<1$ for incomplete information\}, while that information can not be described in IFL because in IFL the components $\mathrm{T}$ (truth), I (indeterminacy), F (falsehood) are restricted either to $\mathrm{t}+\mathrm{I}+\mathrm{f}=1$ or to $\mathrm{t}^{2}+\mathrm{f}^{2} \leq 1$, if $\mathrm{T}, \mathrm{I}, \mathrm{F}$ are all reduced to the points $t, i$, f respectively, or to : $\sup \mathrm{T}+\sup \mathrm{I}+\sup \mathrm{F}=1$ if T, I, F are subsets of $[0,1]$

c) In NL the components T, I, F can also be non-standard subsets included in the unitary non-standard interval $]^{-} 0,1^{+}[$, not only standard subsets included in the unitary standard interval $[0,1]$ as in IFL.

d) NL, like dialetheism, can describe paradoxes, $\mathrm{NL}($ paradox $)=(1, \mathrm{I}, 1)$, while IFL can not describe a paradox because the sum of components should be 1 in IFL.

\section{D) The road to the Neutrosophic Logic (a historical view):}

The Classical Logic, also called Bivalent Logic for taking only two values $\{0,1\}$, or Boolean Logic from British mathematician George Boole (1815-64), was named by the philosopher Quine (1981) "sweet simplicity". Peirce, before 1910, developed a semantics for three-valued logic in an unpublished note, but Emil Post's dissertation (1920s) is cited 
for originating the three-valued logic. Here " 1 " is used for truth, " $1 / 2$ " for indeterminacy, and " 0 " for falsehood. Also, Reichenbach, leader of the logical empiricism, studied it. The three-valued logic was employed by Halld n (1949), K rner (1960), Tye (1994) to solve Sorites Paradoxes. They used truth tables, such as Kleene's, but everything depended on the definition of validity.

A three-valued paraconsistent system (LP) has the values: 'true', 'false', and 'both true and false'. The ancient Indian metaphysics considered four possible values of a statement: 'true (only)', 'false (only)', 'both true and false', and 'neither true nor false'; J. M. Dunn (1976) formalized this in a fourvalued paraconsistent system as his First Degree Entailment semantics;

The Buddhist logic added a fifth value to the previous ones, 'none of these' (called catushkoti).

In order to clarify the anomalies in science, Rugina (1949, 1981) proposed an original method, starting first from an economic point of view but generalizing it to any science, to study the equilibrium and disequilibrium of systems. His Orientation Table comprises seven basic models:

Model $\mathrm{M}_{1}$ (which is $100 \%$ stable)

Model $\mathrm{M}_{2}$ (which is $95 \%$ stable, and $5 \%$ unstable)

Model $\mathrm{M}_{3}$ (which is $65 \%$ stable, and $35 \%$ unstable)

Model $\mathrm{M}_{4}$ (which is $50 \%$ stable, and $50 \%$ unstable)

Model $\mathrm{M}_{5}$ (which is $35 \%$ stable, and $65 \%$ unstable)

Model $\mathrm{M}_{6}$ (which is $5 \%$ stable, and $95 \%$ unstable)

Model $\mathrm{M}_{7}$ (which is $100 \%$ unstable)

He gives Orientation Tables for Physical Sciences and Mechanics (Rugina 1989), for the Theory of Probability, for what he called Integrated Logic, and generally for any Natural or Social Science (Rugina 1989). This is a Seven-Valued Logic.

The $\left\{0, a_{1}, \ldots, a_{n}, 1\right\}$ Multi-Valued, or Plurivalent, Logic was develop by Łukasiewicz, while Post originated the m-valued calculus. 
The many-valued logic was replaced by Goguen (1969) and Zadeh(1975) with an Infinite-Valued Logic (of continuum power, as in the classical mathematical analysis and classical probability) called Fuzzy Logic, where the truth-value can be any number in the closed unit interval $[0,1]$. The Fuzzy Set was introduced by Zadeh in 1965.

Rugina (1989) defines an anomaly as "a deviation from a position of stable equilibrium represented by Model $\mathrm{M}_{1}$ ", and he proposes a Universal Hypothesis of Duality:

"The physical universe in which we are living, including human society and the world of ideas, all are composed in different and changeable proportions of stable (equilibrium) and unstable (disequilibrium) elements, forces, institutions, behavior and value" and a General Possibility Theorem:

"There is an unlimited number of possible combinations or systems in logic and other sciences".

According to the last assertions one can extend Rugina's Orientation Table in the way that any system in each science is $\mathrm{s} \%$ stable and $\mathrm{u} \%$ unstable, with $\mathrm{s}+\mathrm{u}=100$ and both parameters $0 \leq \mathrm{s}, \mathrm{u} \leq 100$, somehow getting to a fuzzy approach.

But, because each system has hidden features and behaviors, and there would always be unexpected occurring conditions we are not able to control - we mean the indeterminacy plays a role as well, a better approach would be the Neutrosophic Model:

Any system in each science is $\mathrm{s} \%$ stable, $\mathrm{i} \%$ indeterminate, and $\mathrm{u} \%$ unstable, with $\mathrm{s}+\mathrm{i}+\mathrm{u}=100$ and all three parameters $0 \leq \mathrm{s}, \mathrm{i}, \mathrm{u} \leq 100$.

Therefore, we finally generalize the fuzzy logic to a transcendental logic, called "neutrosophic logic": where the interval $[0,1]$ is exceeded, i.e. , the percentages of truth, indeterminacy, and falsity are approximated by non-standard subsets - not by single numbers, and these subsets may overlap and exceed the unit interval in the sense of the nonstandard analysis; also the superior sums and inferior sum, $\left.\mathrm{n}_{\text {sup }}=\sup \mathrm{T}+\sup \mathrm{I}+\sup \mathrm{F} \in\right]^{-} 0,3^{+}$[, may be as high as 3 or 
$3^{+}$, while $\left.n_{\text {inf }}=\inf \mathrm{T}+\inf \mathrm{I}+\inf \mathrm{F} \in\right]^{-} 0,3^{+}[$, may be as low as 0 or ${ }^{-} 0$.

Generally speaking, passing from the attribute "classical" (traditional) to the attribute "modern" (in literature, arts, and philosophy today one says today "postmodern") one invalidates many theorems. Voltaire (1694-1778), a French writer and philosopher, asserted that "the laws in arts are made in order to encroach upon them". Therefore, in neutrosophic logic most of the classical logic laws and its properties are not preserved. Although at a first look neutrosophic logic appears counterintuitive, maybe abnormal, because the neutrosophic-truth values of a proposition A, NL(A), may even be $(1,1,1)$, i.e. a proposition can completely be true and false and indeterminate at the same time, studying the paradoxes one soon observes that it is intuitive.

The idea of tripartition (truth, falsehood, indeterminacy) appeared in 1764 when J. H. Lambert investigated the credibility of one witness affected by the contrary testimony of another. He generalized Hooper's rule of combination of evidence (1680s), which was a Non-Bayesian approach to find a probabilistic model. Koopman in 1940s introduced the notions of lower and upper probability, followed by Good, and Dempster (1967) gave a rule of combining two arguments. Shafer (1976) extended it to the Dempster-Shafer Theory of Belief Functions by defining the Belief and Plausibility functions and using the rule of inference of Dempster for combining two evidences proceeding from two different sources. Belief function is a connection between fuzzy reasoning and probability. The Dempster-Shafer Theory of Belief Functions is a generalization of the Bayesian Probability (Bayes 1760s, Laplace 1780s); this uses the mathematical probability in a more general way, and is based on probabilistic combination of evidence in artificial intelligence.

In Lambert "there is a chance $p$ that the witness will be faithful and accurate, a chance $\mathrm{q}$ that he will be mendacious, 
and a chance 1-p-q that he will simply be careless" [apud Shafer (1986)]. Therefore three components: accurate, mendacious, careless, which add up to 1 .

Van Fraassen introduced the supervaluation semantics in his attempt to solve the Sorites paradoxes, followed by Dummett (1975) and Fine (1975). They all tripartitioned, considering a vague predicate which, having border cases, is undefined for these border cases. Van Fraassen took the vague predicate 'heap' and extended it positively to those objects to which the predicate definitively applies and negatively to those objects to which it definitively doesn't apply. The remaining objects border was called penumbra. A sharp boundary between these two extensions does not exist for a soritical predicate. Inductive reasoning is no longer valid too; if $\mathrm{S}$ is a Sorites predicate, the proposition " $\exists \mathrm{n}\left(\mathrm{Sa}_{n} \& \neg \mathrm{Sa}_{\mathrm{n}+1}\right)$ " is false. Thus, the predicate Heap $($ positive extension $)=$ true, Heap $($ negative extension $)=$ false, Heap (penumbra) $=$ indeterminate.

Narinyani (1980) used the tripartition to define what he called the "indefinite set", and Atanassov (1982) continued on tripartition and gave five generalizations of the fuzzy set, studied their properties and applications to the neural networks in medicine.

This neutrosophic logic is the (first) attempt to unify many logics in a single field. However, sometimes a too large generalization may have no practical impact. Such unification theories, or attempts, have been done in the history of sciences:

a) Felix Klein (1872), in his Erlangen program, in geometry, has proposed a common standpoint from which various branches of geometries could be reorganized, interpreted, i.e.:

Given a manifold and a group of transformations of the manifold, to study the manifold configurations with respect to those features that are not altered by the 
transformations of the group (Klein 1893, p. 67; apud Torretti 1999).

b) Einstein tried in physics to build a Unifying Field Theory that seeks to unite the properties of gravitational, electromagnetic, weak, and strong interactions so that a single set of equations can be used to predict all their characteristics; whether such a theory may be developed it is not known at the present (Illingworth 1991, p. 504).

c) Also, one mentions the Grand Unified Theory, which is a unified quantum field theory of the electromagnetic, weak, and strong interactions (Illingworth 1991, p. 200).

Let's borrow from the modal logic the notion of "world", which is a semantic device of what the world might have been like. Then, one says that the neutrosophic truth-value of a statement $\mathrm{A}, \mathrm{NL}_{\mathrm{t}}(\mathrm{A})=1^{+}$if A is 'true in all possible worlds' (syntagme first used by Leibniz) and all conjunctures, that one may call "absolute truth" (in the modal logic it was named necessary truth, Dinulescu-Câmpina (2000) names it 'intangible absolute truth' ), whereas $\mathrm{NL}_{\mathrm{t}}(\mathrm{A})=1$ if $\mathrm{A}$ is true in at least one world at some conjuncture, we call this "relative truth" because it is related to a 'specific' world and a specific conjuncture (in the modal logic it was named possible truth). Because each 'world' is dynamic, depending on an ensemble of parameters, we introduce the sub-category 'conjuncture' within it to reflect a particular state of the world.

How can we differentiate $<$ the truth behind the truth $>$ ? What about the <metaphoric truth>, which frequently occurs in the humanistic field? Let's take the proposition " $99 \%$ of the politicians are crooked" (Sonnabend 1997, Problem 29, p. 25). "No," somebody furiously comments, " $100 \%$ of the politicians are crooked, even more!" How do we interpret this "even more" (than $100 \%$ ), i. e. more than the truth?

One attempts to formalize. For $n \geq 1$ one defines the "n-level relative truth" of the statement $\mathrm{A}$ if the statement is true in at least $\mathrm{n}$ distinct worlds, and similarly "countable-" or 
"uncountable-level relative truth" as gradual degrees between "first-level relative truth" (1) and "absolute truth" $\left(1^{+}\right)$in the monad $\mu\left(1^{+}\right)$. Analogue definitions one gets by substituting "truth" with "falsehood" or "indeterminacy" in the above.

In largo sensu the notion "world" depends on parameters, such as: space, time, continuity, movement, modality, (meta)language levels, interpretation, abstraction, (higher-order) quantification, predication, complement constructions, subjectivity, context, circumstances, etc. Pierre d'Ailly upholds that the truth-value of a proposition depends on the sense, on the metaphysical level, on the language and meta-language; the auto-reflexive propositions (with reflection on themselves) depend on the mode of representation (objective/subjective, formal/informal, real/mental).

In a formal way, let's consider the world $\mathrm{W}$ as being generated by the formal system FS. One says that statement A belongs to the world $\mathrm{W}$ if A is a well-formed formula (wff) in $\mathrm{W}$, i.e. a string of symbols from the alphabet of $\mathrm{W}$ that conforms to the grammar of the formal language endowing W. The grammar is conceived as a set of functions (formation rules) whose inputs are symbols strings and outputs "yes" or "no". A formal system comprises a formal language (alphabet and grammar) and a deductive apparatus (axioms and/or rules of inference). In a formal system the rules of inference are syntactically and typographically formal in nature, without reference to the meaning of the strings they manipulate.

Similarly for the neutrosophic falsehood-value, $\mathrm{NL}_{\mathrm{f}}(\mathrm{A})=1^{+}$if the statement $\mathrm{A}$ is false in all possible worlds, we call it "absolute falsehood", whereas $\operatorname{NL}_{\mathrm{f}}(\mathrm{A})=1$ if the statement $\mathrm{A}$ is false in at least one world, we call it "relative falsehood". Also, the neutrosophic indeterminacy-value $\mathrm{NL}_{\mathrm{i}}(\mathrm{A})=1^{+}$if the statement $\mathrm{A}$ is indeterminate in all possible worlds, we call it "absolute indeterminacy", whereas $\mathrm{NL}_{\mathrm{i}}(\mathrm{A})=1$ if the statement A is indeterminate in at least one world, we call it "relative indeterminacy". 
On the other hand, $\mathrm{NL}_{\mathrm{t}}(\mathrm{A})=-0$ if $\mathrm{A}$ is false in all possible world, whereas $\mathrm{NL}_{t}(\mathrm{~A})=0$ if $\mathrm{A}$ is false in at least one world; $\mathrm{NL}_{\mathrm{f}}(\mathrm{A})=-0$ if $\mathrm{A}$ is true in all possible world, whereas $\mathrm{NL}_{\mathrm{f}}(\mathrm{A})=0$ if $\mathrm{A}$ is true in at least one world; and $\mathrm{NL}_{\mathrm{i}}(\mathrm{A})=-0$ if $A$ is indeterminate in no possible world, whereas $\mathrm{NL}_{\mathrm{i}}(\mathrm{A})=0$ if $\mathrm{A}$ is not indeterminate in at least one world.

The ${ }^{-} 0$ and $1^{+}$monads leave room for degrees of super-truth (truth whose values are greater than 1), super-falsehood, and super-indeterminacy.

Here there are some corner cases:

There are tautologies, some of the form "B is B", for which NL $(B)=\left(1^{+},-0,-0\right)$, and contradictions, some of the form "C is not C", for which $\mathrm{NL}(\mathrm{C})=\left({ }^{-} 0,{ }^{-} 0,1^{+}\right)$.

While for a paradox, $\mathrm{P}, \mathrm{NL}(\mathrm{P})=(1,1,1)$. Let's take the Epimenides Paradox, also called the Liar Paradox, "This very statement is false". If it is true then it is false, and if it is false then it is true. But the previous reasoning, due to the contradictory results, indicates a high indeterminacy too. The paradox is the only proposition true and false in the same time in the same world, and indeterminate as well!

Let's take the Grelling's Paradox, also called the heterological paradox [Suber, 1999], "If an adjective truly describes itself, call it 'autological', otherwise call it 'heterological'. Is 'heterological' heterological? "Similarly, if it is, then it is not; and if it is not, then it is.

For a not well-formed formula, nwff, i.e. a string of symbols which do not conform to the syntax of the given logic, $\mathrm{NL}$ (nwf) $=\mathrm{n} / \mathrm{a}$ (undefined). A proposition which may not be considered a proposition was called by the logician Paulus Venetus flatus voci. NL (flatus voci) $=\mathrm{n} / \mathrm{a}$.

\section{E) Comments on Neutrosophic Logic in Comparison to Other Logics:}

In a two-valued system, one regards all the designated values as species of truth and all the anti-designated values as species 
of falsehood, with truth-value (or falsehood-value) gaps between designated and anti-designated values. In the neutrosophic system one stipulates the non-designated values as species of indeterminacy and, thus, each neutrosophic consequence has degrees of designated, non-designated, and anti-designated values.

Of course, the Law of Excluded Middle (a proposition is either true or false) does not hold in a neutrosophic system.

The Contradiction Law, that no $<\mathrm{A}>$ is $<$ Non- $\mathrm{A}>$ does not hold too. $\mathrm{NL}(<\mathrm{A}>)$ may be equivalent with $\mathrm{NL}(<$ Non- $\mathrm{A}>)$ and often they at least overlap. Neither the law of Reductio ad absurdum (or method of indirect proof):

$$
\left.\left.\left.(\mathrm{A} \supset\urcorner_{\urcorner} \mathrm{A}\right) \supset\right\urcorner \mathrm{A} \text { and }(\urcorner \mathrm{A} \supset \mathrm{A}\right) \supset \mathrm{A}
$$

Some tautologies (propositions logically necessary, or true in virtue of form) in the classical logic might not be tautologies (absolute truth-value propositions) in the neutrosophic logic and, mutatis mutandis, some contradictions (propositions logically impossible, or false in virtue of form) in the classical logic might not be contradictions (absolute falsehood-value propositions) in the neutrosophic logic.

- The mixed hypothetical syllogism Modus Ponens,

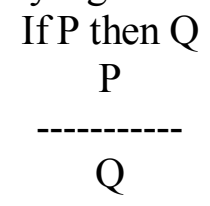

- The mixed hypothetical syllogism Modus Tollens,

$$
\text { If } P \text { then } Q
$$

Non Q

Non P 
- The Inclusive (Weak) Disjunctive Syllogism:

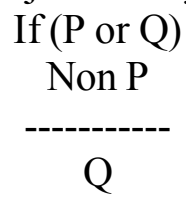

- The Exclusive (Strong) Disjunctive Syllogism:

$$
\begin{gathered}
\text { If (either P or Q) } \\
\text { Non P }
\end{gathered}
$$

Q

- Hypothetical Syllogism,

$$
\begin{aligned}
& \text { If } P \text { then } Q \\
& \text { If } Q \text { then } R \\
& ----- \\
& \text { If } P \text { then } R
\end{aligned}
$$

- Constructive Dilemma,

$$
\begin{gathered}
\mathrm{P} \text { or } \mathrm{Q} \\
\text { If } \mathrm{P} \text { then } \mathrm{R} \\
\text { If } \mathrm{Q} \text { then } \mathrm{R} \\
\text {-------- } \\
\mathrm{R}
\end{gathered}
$$

- Destructive Dilemma,

$$
\begin{gathered}
\text { P or Q } \\
\text { Non P } \\
---- \\
\text { Q }
\end{gathered}
$$

The Polysyllogism, which is formed by many syllogisms such that the conclusion of one becomes a premise of another, and the Nested Arguments, a chainlike where the conclusion of an argument forms the premise of another where intermediate conclusions are typically left out, are not valid anymore in the neutrosophic logic, but they acquire a more complex form. 
Also, the classical entailment, which is the effect that a proposition Q is a necessary consequence of another proposition $\mathrm{P}, \mathrm{P} \tau \mathrm{Q}$, partially works in the neutrosophic logic. Neither the informal fish-hook symbol, ---], used to show that a proposition $\mathrm{Q}$ is an accidental consequence of a proposition $\mathrm{P}, \mathrm{P}$---] Q, works.

Is it possible in the neutrosophic predicate calculus to transform each formula into an equivalent in prenex form ${ }^{11}$ one using the prenex operations? Prenex (normal) form means a formula formalized as follows:

$$
\left(\mathrm{Qx}_{1}\right)\left(\mathrm{Qx}_{2}\right) \ldots\left(\mathrm{Qx_{ \textrm {n } }}\right) \mathrm{S}
$$

Where "Q" is a universal or existential quantifier, the variables $\mathrm{x}_{1}, \mathrm{x}_{2}, \ldots, \mathrm{x}_{\mathrm{n}}$ are distinct, and $\mathrm{S}$ is an open sentence (a well-formed expression containing a free variable). Prenex operation is any operation which transforms any well-formed formula into equivalent in prenex form formula; for example,

$$
(\exists x) A x \rightarrow B \equiv(\forall x)(A x \rightarrow B)
$$

In the classical predicate calculus any well-formed formula can be transformed into a prenex form formula.

The double negation, $\left.\urcorner_{7} \mathrm{~A}\right) \equiv \mathrm{A}$, which is not valid in intuitionistic logic, is not valid in the neutrosophic logic if one considers the negation operator $\eta_{1}(\mathrm{~A})=1 \Theta \mathrm{NL}(\mathrm{A})$, but it is valid for the negation operator $\eta_{2}(\mathrm{~A})=(\mathrm{F}, \mathrm{I}, \mathrm{T})$, where $\mathrm{NL}(\mathrm{A})=(\mathrm{T}, \mathrm{I}, \mathrm{F})$.

Neutrosophic Logic admits non-trivial inconsistent theories.

In stead of saying "a sentence holds (or is assertable)" as in classical logic, one extends to "a sentence $\mathrm{p} \%$ holds (or is $\mathrm{p} \%$ assertable)" in neutrosophic logic. In a more formalized way, a sentence (T, I, F)\% holds [or is (T, I, F)\% assertable].

A neutrosophic predicate is a vague, incomplete, or not well known attribute, property or function of a subject. It is a kind of three-valued set function. If a predicate is applied to more than one subject, it is called neutrosophic relation.

An example: "Andrew is tall'. The predicate "tall" is imprecise. Andrew is maybe tall according to Linda, but short 
in Jack's opinion, however his tallness is unknown to David. Everybody judges him in terms of his/her own tallness and acquaintance of him.

The neutrosophic set and logic attempt to better model the non-determinism. They try:

- to represent the paradoxical results even in science not talking in the humanistic where the paradox is very common;

- to evaluate the peculiarities;

- to illustrate the contradictions and conflicting theories, each true from a specific point of view, false from another one, and perhaps indeterminate from a third perspective;

- to catch the mysterious world of the atom, where the determinism fails; in quantum mechanics we are dealing with systems having an infinite number of degrees of freedom;

- to study submicroscopic particles which behave nonNewtonianly, and some macroscopic phenomena which behave in nearly similar way.

In physics, the light is at once a wave and a particle (photon). Two contradictory theories were both proven true:

The first one, Wave Theory (Maxwell, Huygens, and Fresnel), says that light is a wave due to the interference: two beams of light could cross each other without suffering any damage. The second one, Particle Theory (Newton, Hertz, Lenard, Planck, Einstein), says that light is corpuscular, due to the photoelectric effect that ultraviolet light is able to evaporate electrons from metal surfaces and to the manner in which light bounces off electrons.

De Broglie reconciled both theories proving that light is a matter wave! Matter and radiation are at the same time waves and particles.

Let L1(x) be the predicate: "X is of corpuscular nature", and $\mathrm{L} 2(\mathrm{x})$ the predicate: " $\mathrm{X}$ is of wave nature".

L2( $\mathrm{x})$ is the opposite of L1(x), nonetheless L1 (light) = true and L2 (light) = true simultaneously. 
Also, there exist four different Atom Theories: of Bohr, Heisenberg, Dirac, and Schrödinger respectively, each of them plausibly true in certain conditions (hypotheses).

Another example, from Maxwell's equations an electron does radiate energy when orbits the nucleus, from Bohr's theory an electron does not radiate energy when orbits the nucleus, and both propositions are proved true with our today's knowledge.

Falsehood is infinite, and truthhood quite alike; in between, at different degrees, indeterminacy as well.

In the neutrosophic theory:

Between being and nothingness

Existence and nonexistence

Geniality and mediocrity

Certainty and uncertainty

Value and nonvalue

And generally speaking $<\mathrm{A}>$ and $<$ Non-A $>$

There are infinitely many transcendental states.

And not even 'between', but even beyond them.

An infinitude of infinitudes.

They are degrees of neutralities $<$ Neut-A $>$ combined with $<\mathrm{A}>$ and $<$ Non- $\mathrm{A}>$.

In fact there also are steps:

Between being and being

Existence and existence

Geniality and geniality

Possible and possible

Certainty and certainty

Value and value

And generally speaking between $<\mathrm{A}>$ and $<\mathrm{A}>$

The notions, in a pure form, last in themselves only (intrinsicalness), but outside they have an interfusion form.

Infinitude of shades and degrees of differentiation: between white and black there exists an unbounded palette of colors resulted from thousands of combinations among them. All is alternative: progress alternates with setback, development with stagnation and underdevelopment. 
In between objective and subjective there is a plurality of shades.

In between good and bad

In between positive and negative

In between possible and impossible

In between true and false

In between "A" and "Anti-A"

As a neutrosophic ellipse:

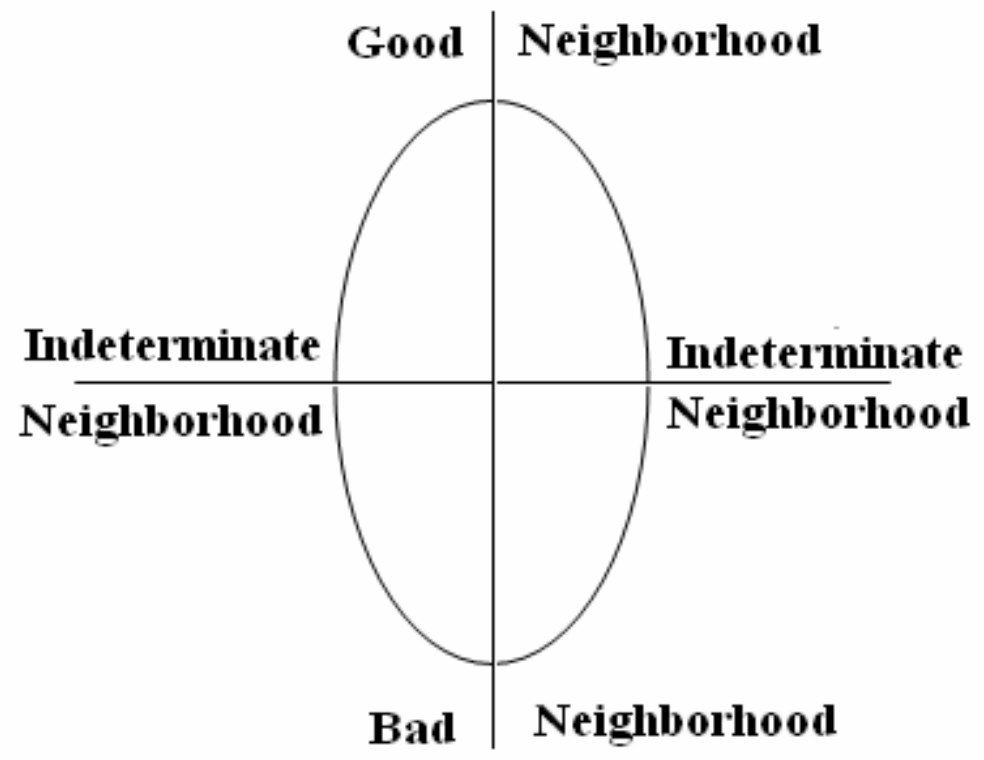

Everything is $\mathrm{g} \%$ good, $\mathrm{i} \%$ indeterminate, and $\mathrm{b} \% \mathrm{bad}$, where $g$ varies in the subset $G$, $i$ varies in the subset $I$, and $b$ varies in the subset $B$, and G, I, B are included in $]^{-} 0,1^{+}[$.

Besides Diderot's dialectics on good and bad ("Rameau's Nephew", 1772), any act has its "god", "indeterminate", and of "bad" as well incorporated.

Rodolph Carnap said:

"Metaphysical propositions are neither true nor false, because they assert nothing; they contain neither knowledge nor error (...)". 
Hence, there are infinitely many states between "Good" and "Bad", and generally speaking between "A" and "Anti-A" (and even beyond them), like on the real number line:

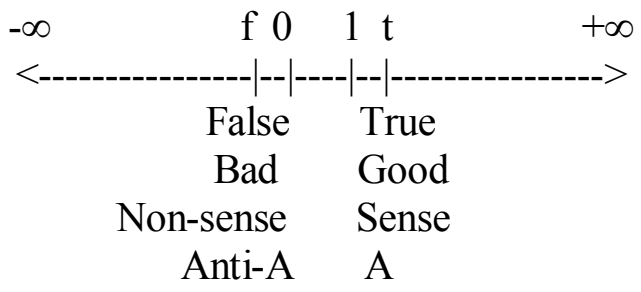

$f$ is the absolute falsity $(f<0), t$ the absolute truth $(t>1)$. In between each oppositing pair, normally in a vicinity of 0.5 , are being set up the neutralities.

Even if an act apparently looks to be only good or only bad, other hidden sides might be sought. The ratios:

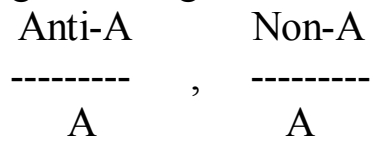

vary indefinitely. Are they transfinite?

If a statement is $30 \% \mathrm{~T}$ (true) and $60 \% \mathrm{I}$ (indeterminate), then it maybe, for example, $15 \% \mathrm{~F}$ (false). This is somehow alethic, meaning to simultaneously pertain to truthhood and falsehood, or to truthhood and indeterminacy, or to falsehood and indeterminacy, or even to all three components. More general, if a statement is $30 \% \mathrm{~T}$ and $60 \% \mathrm{I}$, it may be between $5-20 \% \mathrm{~F}$ or $25 \% \mathrm{~F}$.

\section{F) Comparison between Fuzzy Logic and Neutrosophic}

\section{Logic:}

The neutrosophic connectives have a better truth-value definition approach to the real-world systems than the fuzzy connectives. They are defined on triple non-standard subsets included in the non-standard unit interval ] $0,1^{+}$[, while in fuzzy logic they are defined on the closed interval $[0,1]$. n_sup is not restricted to 1 , but it's enlarged to a monad $\mu\left(3^{+}\right)$, i.e. a set 
of hyper-real numbers; similarly, n_inf may be as low as $\mu\left({ }^{-0}\right)$ , not as 0 .

A paradox, which is true and false in the same time, can not be evaluate in fuzzy logic, because the sum of components should add up to 1, but it is allowed in neutrosophic logic because NL(paradox) maybe $(1,1,1)$.

In opposition to Fuzzy Logic, if a proposition $<A>$ is $t \%$ true, doesn't necessarily mean it is (100-t) $\%$ false. A better approach is $\mathrm{t} \%$ true, $\mathrm{f} \%$ false, and $\mathrm{i} \%$ indeterminate as in Intuitionistic Fuzzy Logic (Atanassov), where $t \in T, i \in I, f \in F$, even more general, with $n_{-}$sup $\leq 3^{+}$and $n_{-}$inf $\geq^{-} 0$.

\section{G) Neutrosophical Modal Logic:}

In modal logic, the primitive operators $\diamond$ 'it is possible that' and $\square$ 'it is necessary that' can be defined by:

$$
t_{-} \inf (\diamond \mathrm{A})>0 \text {, }
$$

and, because $\square$ A could be regarded as $\neg(\diamond \neg A)$,

$$
\mathrm{t}_{-} \sup (\square \mathrm{A})<1 \text {. }
$$

\section{H) Applications:}

Neutrosophic logic is useful in the real-world systems for designing control logic, and may work in quantum mechanics.

\# The candidate $\mathrm{C}$, who runs for election in a metropolis $\mathrm{M}$ of p people with right to vote, will win.

This proposition is, say, 20-25\% true (percentage of people voting for him), 35-45\% false (percentage of people voting against him), and $40 \%$ or $50 \%$ indeterminate (percentage of people not coming to the ballot box, or giving a blank vote - not selecting anyone, or giving a negative vote - cutting all candidates on the list).

\# Tomorrow it will rain.

This proposition is, say, $50 \%$ true according to meteorologists who have investigated the past years' 
weather, between $20-30 \%$ false according to today's very sunny and droughty summer, and $40 \%$ undecided.

\# This is a heap.

As an application to the Sorites paradoxes, we may now say that this proposition is $80 \%$ true, $40 \%$ false, and $25-35 \%$ indeterminate (the neutrality comes for we don't know exactly where is the difference between a heap and a non-heap; and, if we approximate the border, our 'accuracy' is subjective). Vagueness plays here an important role.

We are not able to distinguish the difference between yellow and red as well if a continuum spectrum of colors is painted on a wall imperceptibly changing from one into another.

We would be able to say at a given moment that a section is both yellow and red in the same time, or neither one!

A paradox within a Sorites paradox: a frontal bald man, with a hair high density on the remaining region of his head, may have more hairs on his head that another man who is not bald but the skin surface of his head and the hair density are smaller than the previous one.

\section{I) Definition of Neutrosophic Logical Connectives:}

The connectives (rules of inference, or operators), in any non-bivalent logic, can be defined in various ways, giving rise to lots of distinct logics. For example, in three-valued logic, where three possible values are possible: true, false, or undecided, there are 3072 such logics! (Weisstein, 1998) A single change in one of any connective's truth table is enough to form a (completely) different logic.

Similarly, there are many ways to construct such connectives according to each particular problem to solve; here we present the easiest ones:

One notes the neutrosophic logical values of the propositions $\mathrm{A}_{1}$ and $\mathrm{A}_{2}$ by $\mathrm{NL}\left(\mathrm{A}_{1}\right)=\left(\mathrm{T} 1, \mathrm{I}_{1}, \mathrm{~F}_{1}\right)$ and $\mathrm{NL}\left(\mathrm{A}_{2}\right)=\left(\mathrm{T} 2, \mathrm{I}_{2}, \mathrm{~F}_{2}\right)$. 
1. Negation:

$$
\operatorname{NL}\left({ }_{\rceil} \mathrm{A}_{1}\right)=\left(\{1\} \ominus \mathrm{T}_{1},\{1\} \ominus \mathrm{I}_{1},\{1\} \ominus \mathrm{F}_{1}\right)
$$

\section{Conjunction:}

$\mathrm{NL}\left(\mathrm{A}_{1} \wedge \mathrm{A}_{2}\right)=\left(\mathrm{T} 1 \odot \mathrm{T}_{2}, \mathrm{I}_{1} \odot \mathrm{I}_{2}, \mathrm{~F}_{1} \odot \mathrm{F}_{2}\right)$

(And, in a similar way, generalized for $\mathrm{n}$ propositions)

\section{Weak or inclusive disjunction:}

$\mathrm{NL}\left(\mathrm{A} 1 \vee \mathrm{A}_{2}\right)=\left(\mathrm{T} 1 \oplus \mathrm{T}_{2} \ominus \mathrm{T}_{1} \odot \mathrm{T}_{2}, \mathrm{I}_{1} \oplus \mathrm{I}_{2} \ominus \mathrm{I}_{1} \odot \mathrm{I}_{2}\right.$,

$\left.\mathrm{F}_{1} \oplus \mathrm{F}_{2} \ominus \mathrm{F}_{1} \odot \mathrm{F}_{2}\right)$

(And, in a similar way, generalized for $\mathrm{n}$ propositions)

\section{Strong or exclusive disjunction:}

$\operatorname{NL}\left(A_{1} \underline{\vee} A_{2}\right)=$

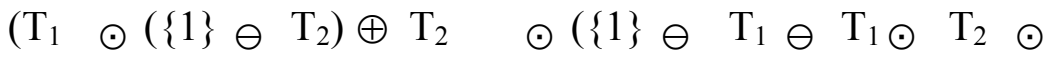

$\left(\{1\} \ominus \mathrm{T}_{1}\right) \odot\left(\{1\} \ominus \mathrm{T}_{2}\right), \mathrm{I}_{1} \odot\left(\{1\} \ominus \mathrm{I}_{2} \oplus \mathrm{I}_{2} \odot(\{1\} \ominus\right.$

$\left.\mathrm{I}_{1}\right) \quad \ominus \quad \mathrm{I}_{1} \odot \mathrm{I}_{2} \odot\left(\{1\} \quad \ominus \mathrm{I}_{1}\right) \odot\left(\{1\} \ominus \mathrm{I}_{2}\right), \quad \mathrm{F}_{1} \odot$

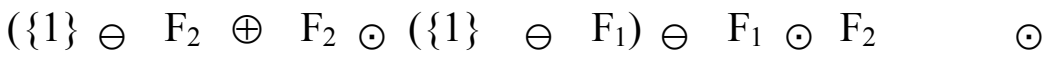

$\left(\{1\} \ominus \mathrm{F}_{1} \odot\left(\{1\} \ominus \mathrm{F}_{2}\right)\right)$.

(And, in a similar way, generalized for $\mathrm{n}$ propositions)

\section{Material conditional (implication):}

$\mathrm{NL}\left(\mathrm{A} 1 \mapsto \mathrm{A}_{2}\right)=\left(\{1\} \ominus \mathrm{T}_{1} \oplus \mathrm{T}_{1} \odot \mathrm{T}_{2},\{1\} \ominus \mathrm{I}_{1} \oplus \mathrm{I}_{1} \odot\right.$

$\left.\mathrm{I}_{2},\{1\} \ominus \mathrm{F}_{1} \oplus \mathrm{F}_{1} \odot \mathrm{F}_{2}\right)$

6. Material biconditional (equivalence):

$\mathrm{NL}\left(\mathrm{A}_{1} \leftrightarrow \mathrm{A}_{2}\right)=$

$$
\left(\left(\{1\} \ominus \mathrm{T}_{1} \oplus \mathrm{T}_{1} \odot \mathrm{T}_{2}\right) \odot\left(\{1\} \ominus \mathrm{T}_{2} \oplus \mathrm{T}_{1} \odot \mathrm{T}_{2}\right),\right.
$$$$
\left(\{1\} \ominus \mathrm{I}_{1} \oplus \mathrm{I}_{1} \odot \mathrm{I}_{2}\right) \odot\left(\{1\} \ominus \mathrm{I}_{2} \oplus \mathrm{I}_{1} \odot \mathrm{I}_{2}\right) \text {, }
$$

$\left.\left(\{1\} \ominus \mathrm{F}_{1} \oplus \mathrm{F}_{1} \odot \mathrm{F}_{2}\right) \odot\left(\{1\} \ominus \mathrm{F}_{2} \oplus \mathrm{F}_{1} \odot \mathrm{F}_{2}\right)\right)$. 


\section{Sheffer's connector:}

$$
\begin{aligned}
& \left.\left.\operatorname{NL}\left(A_{1} \mid A_{2}\right)=N L(\urcorner A_{1} \vee\right\urcorner A_{2}\right)= \\
& \quad\left(\{1\} \ominus T_{1} \odot T_{2},\{1\} \ominus I_{1} \odot I_{2},\{1\} \ominus F_{1} \odot F_{2}\right) .
\end{aligned}
$$

\section{Peirce's connector:}

$$
\begin{aligned}
& \left.\mathrm{NL}\left(\mathrm{A}_{1} \downarrow \mathrm{A}_{2}\right)=\mathrm{NL}(\urcorner \mathrm{A}_{1} \wedge{ }_{\urcorner} \mathrm{A}_{2}\right)= \\
& \left(\left(\{1\} \ominus \mathrm{T}_{1}\right) \odot\left(\{1\} \ominus \mathrm{T}_{2}\right),\left(\{1\} \ominus \mathrm{I}_{1}\right) \odot\right. \\
& \left.\left(\{1\} \ominus \mathrm{I}_{2}\right), \quad\left(\{1\} \ominus \mathrm{F}_{1}\right) \odot\left(\{1\} \ominus \mathrm{F}_{2}\right)\right) .
\end{aligned}
$$

In the dialetheism it works the metaphysical thesis that some contradictions are true. In the neutrosophic logic there are contradictions denoted by $\mathrm{t}=\mathrm{f}=1$, which means $100 \%$ true and $100 \%$ false in the same time; even more, it is possible to have propositions which are, say, $70 \%$ true and $60 \%$ false (considering different sources or criteria) - the truth- and falsehood-components overlap (especially in pseudo paradoxes), while in the fuzzy logic it is not - because the components should sum to $100 \%$, i.e. $70 \%$ true and $30 \%$ false.

What is the logic of the logic? We study the apparently illogic of the logic, as well as the logic of the illogic.

There exist two main types of truth: the true truth and the false truth, besides the intermediate shades of truth. And similarly for the falsity: the true falsity and the false falsity, beside the intermediate shades of falsity.

The neutrosophic logic unifies many logics; it is like Felix Klein's program in geometry, or Einstein's unified field in physics.

\subsection{Paradoxism Used in Science}

Paradoxism naturally arises and manifests. It is in folklore, humanistic fields, and even more. Through paradoxist 
experiments, one brings new literary, artistic, philosophical, or scientific terms, new procedures, methods, or even algorithms of creation. Surprisingly, the paradoxism found its use in science too!

Let us see an example from cybernetics, regarding the fusion of information. The robots dispose of multi-sensors which receive informations that must be processed, but these informations are often contradictory in a smaller or higher degree. The fusion of such conflicting, paradoxist informations in science is an old problem not entirely solved by the existing theories of Dempster-Shaffer, Dubois-Prade's, Smets' TBM, Yager's, Zadeh's (fuzzy sets), etc. The robot needs to process these informations and gets alone a decision. Here it is the paradoxism.

Similarly in military applications: for target tracking. Or processing more or less conflicting medical images in order to diagnostic diseases, or combining conflicting land agriculture images taken from satellite.

During the second half of the 20th century, several new and interesting mathematical theories have emerged in parallel with the development of computer science and technology. These theories were aiming to combine many types of information (fuzzy, neutrosophic, uncertain, imprecise, paradoxist, incomplete, paraconsistent, Sorites paradoxes, continuous elements, etc.) provided by different sources (human expertise, sensor measurements, AI expert systems, neural network, quantum theory, economics predictions). One such theory, that permits the combination of paradoxist information, has been called Dezert-Smarandache Theory of Plausible and Paradoxist Reasoning for Data Fusion (2001), developed by Prof. Florentin Smarandache (University of New Mexico, USA) and Dr. Jean Dezert from ONERA (French Airspace Research Agency in Paris).

Several international conferences dedicated to the use of paradoxism in science: 
- The first one, called Applications of Plausible, Paradoxical and Neutrosophic Reasoning for Information Fusion, 8-11 July 2003, at Radisson Hotel, Cairns, Queensland, Australia.

- The second one, called Applications and Advances of Plausible and Paradoxical Reasoning for Data Fusion", June 28-July 1, 2004, in Stockholm, Sweden.

On 5 November 2004, Paradoxism, used in the fusion of conflicting information, was invited to NASA Langley Research Center, in Hampton, Virginia, USA.

Between 16-27 May 2005, Paradoxism, again used in the fusion of conflicting information, was presented at the Advanced Study Institute, Albena, Bulgaria.

Since 2003 until today we participated each year to the International Conferences dedicated to the Information Fusion, organized respectively in Australia, Sweden, USA, Italy, but also we are sponsored by Marcus Evans Inc. to present tutorials about DSmT to Spain and Belgium. 


\section{Notes of Chapter 1}

1. This chapter is written by Florentin Smarandache, and its footnotes are written by Salah Osman.

2. This neutrosophic classification of ideas corresponds with Ibn Miskawayh's classification of the types of lovingness (affection) in his: Politeness of ethics and clearing of races (Tahdhib al-Akhlaq). He classified these types as following:

- What arises quickly and decays quickly (because its cause is the pleasure).

- What arises quickly and decays slowly (because its cause is the good).

- What arises slowly and decays quickly (because its cause is the utility).

- What arises slowly and decays slowly (because it has all the previous causes).

[Ibn Miskawayh is an Arabic historian and philosopher of ethics $(932-1030)]$. See the book in question, ed. by Ibn alKhatib, The Egyptian Press and its Library ( Al-Matba'a alMisriyyah wa Maktabatiha ), Cairo, Egypt, No date, pp. 149 50.

3. In Islam, there is no difference, inconsistency, oppositeness, or contradiction between religion and science, in order to say if the level of science increase, the level of religion decrease, and vice versa. On the contrary, the religion, in Islam, is a science, because it doesn't only depend on feeling or emotion, but also on thinking and speculation. In other words, the Islamic religion is calling for use the mind to get the correct and sufficient proof, not to dependence on guess or blind imitation. Also, the science in Islam is a religion, because the attempt to acquire it is a religious duty for every Muslim. Moreover, the practice of useful science is a worship and struggle (Jihad) in the path of Allah. The powerful evidence for that is the Quranic verse: 
And of people, reptilian and cattle variegation also holds true. Thus, such of Allah's servants as stand in awe of Him are the savants. Allah is Azizun (Almighty) and Ghafurun (Forgiving)

$$
\{\text { Fater }=\text { The Creator, Verse } 28\} \text {. }
$$

Florentin Smarandache has worked as a math professor in Morocco for two years, 1982-4, teaching in French language, when he read the Quran and got familiarized with the Islamic culture, arts and literature. He has even published a Moroccan diary in Romanian, and in French three volumes of avant-garde poetry, one book of math problems for Moroccan students, and one collection of math articles during that period.

4. This law is well known in Islam, it's regarded as one of the call's bases to religion. That's what the following Quranic verse express:

Invite people O Muhammad graciously to the path of Allah, the path of righteousness, with wise, graceful and appropriate inducements meeting them on their own ground and propitiating them, each in his own capacity, to open their hearts' ears and their minds' eyes. Reason with them in a logical, peaceable and gracious manner; Allah, your Creator, knows best those who have wandered from His path of righteousness and He knows best those who steer a straight course and Providence their guide

$$
\{A l-N a h l=\text { The Bees, Verse } 125\} \text {. }
$$

5. There are many sayings of the Prophet Muhammad (Hadiths) that confirm this example, such as: «The permanent little is better than the discontinuous much ».

6. Newton's third law says: "All forces occur in pairs, and these two forces are equal in magnitude and opposite in direction», but the neutrosophic rule confirms that there is an interval that has infinite number of points between action and reaction. These points represent different reactions from action, but they aren't necessary contrary to it.

7. There is no success without failure, because the experiment of failure gives the man an experience for its causes and the ways of avoid it. The Prophet Muhammad said about that: "The believer isn't stung from a burrow twice».

8. The completeness must lead to the deficiency. Compare that with 
the saying of Islamic leader $A l$ - Shãfi'ĩ (Abu Abd Allah Muhammad) (767 - 820): " if any bird flies, and goes up through the air, it must fall down ».

9. This statement similar to another one we reiterate in Arabic world by way of ridicule in front of the case of separation and dispersion among Arabic governments. We say: «Arabs agree to that they don't agree! ».

10.Also compare the maxim of Omar Ibn al - Khattab, the second Caliph of Muslims (581 - 644), where he said: «Who doesn't know the evil is worthy to tumble in its.

11. A formula is said to be in prenex normal form if all the quantifiers which it contains stand together at the beginning, unseparated by negations (or other sentential connectives), and the scope of each quantifier (i.e., the extent of the bracket [ ] following the quantifier) is to the end of the entire formula. In the case of a formula in prenex normal form, the succession of quantifiers at the beginning is called the prefix; the remaining portion contains no quantifiers and is the matrix of the formula. It can be proved that for every formula $\mathrm{A}$ there is a formula $\mathrm{B}$ in prenex normal form such that $\mathrm{A} \equiv \mathrm{B}$ is a theorem; and $\mathrm{B}$ is then called a prenex normal form of A. (Runes, Dictionary of Philosophy, Item: logic, formal, pp. 190 - 191.

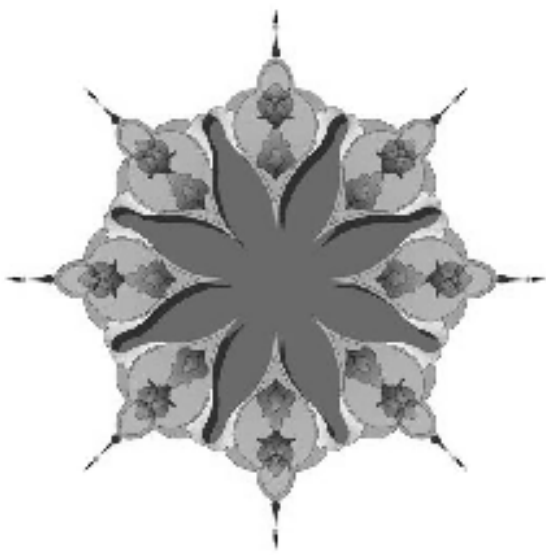




\section{Chapter 2}

\section{Arabic - Islamic Thought: Its Sources and Pillars}

\subsection{Spatial - Temporal Dimension}

This chapter aims to determine the general features of Arabic-Islamic thought in its early centuries: its sources and pillars; motives and aims; problems and main cases. Before that, its specialty that formed what we can call the cultural and cognitive context that is location-bound, i.e., the specialty that granted this thought its distinct place in the course of man's civilizational evolution.

Undoubtedly, this aim was the axis of several and various studies carried out by historians and researchers. They have different cultural identities, and for varied purposes that tend mostly to favoritism. They also include the desire in presenting ready-made and comforting explanations. But in the framework of this book, the aim takes another different dimension. This dimension is to identify and confirm the neutrosophic character of ideas; namely their contradictions, changes, and spectra of neutrality; whether in the general course of their evolution, through their transition from civilization to another, according to the argument of influence and impact; or in their own interactive course of evolution, within the crucible of Arabic-Islamic civilization.

I hope this may contribute in specifying the present moment of Arabic mind, and the significance of external influences that seek to comprehend its cultural legacy and reform it 
within a new system, of which the West is propagating, especially the American West.

That does not mean that we aim to resurrect the whole Arabic past, which contains the good and the bad. We refuse the acceptance of glaring calls to escape from the capture of past, because this past is a part and parcel of Arabic identity however we admit or disapprove. Yet, we had better aim to present a new neutral reading that recognizes the thought through its multiplicity, and discloses the infinite number of possible worlds of ideas. This reading, undoubtedly, will allow us to build new and real bases for the selection and comparison among the legacies of the past, the requirements of the present, and the aspirations of the future.

Let us begin with a local and neutral definition of Arabic (Islamic) thought in terms of its spatial-temporal dimension. The term «Arabic (Islamic) thought» denotes all aspects of mental creative activity of those individuals who lived in a region that roughly extended chronologically from the eight century A.D. (the second century A.H.) to the thirteenth century A.D. (the seventh century A.H.); and geographically from the Iberian Peninsula and North Africa to the Indus Valley and from Southern Arabia to the Caspian Sea - that is, the region covered for most of the period by what we call Islamic civilization, and in which the results of the activities referred to were for the most part expressed in the Arabic language. ${ }^{2}$

A glance at this definition will show that it requires details; the spatial-temporal dimension, for example, may arise questions such as: haven't the Arabs got any civilization before the appearance of Islam?, were they the only builders of that Islamic civilization?, as long as the Islam is still remaining, deep-routed and alive, in the conscience of Arabs, then why their civilization has declined?, and what about the validity of use of the term «East» from the geographical perspective?. 
We may as well ask about the substance of the term "Arabic thought», or about its elements and significance of its correlation with the Arabic language as a sole way of expression: does this language have holiness with its speakers as a language of the Quran?

The researchers differ with each other about the answers of these questions, and we will note through their different answers that there are common parts between any idea, or answer, and its opposite, and there are justifications of the equilibrium among the struggled ideas, according to a neutrosophic scheme that rule out the absolute stability for $<\mathrm{A}>,<$ Non-A $>$, and $<$ Anti-A $>$.

Since the previous questions finally cluster in one class; i.e., the class of modern difference about the designation of philosophical and scientific legacy, written in Arabic, in the name of "Arabic civilization", or "Islamic civilization", or "the civilization of East in medieval centuries", we shall treat all them in the frame of restricted problem, which we can call «the neutrosophism of designation».

\subsection{The Neutrosophism of Designation}

Firstly, we should indicate that the use of the term «East» in the history of civilization is not exactly in accordance with its geographical meaning. As the urban states of Near East had to be named «the South» in Russia, as well as Northern Africa, which is considered as a part of Islamic East, because it is Southern as to Europe!, therefore, our use of the term «East» must be connected with the context.

In this respect, the Russian historian "Vasily Vladimirovich Bartold", (1869 - 1930) indicated that the first use of the word «East», to mean «urban states», refers to the era of Roman State. Before that, there was no classification of the world's people from the Greek view, especially Aristotle's view, except the people of hot South; who are urban but lack to courage, and the people of cold European North; who are 
courageous but haven't got the ability of urbanization and management of State. The Greek people reside in the intermediate area between the two groups, where the nature of their countries allows them to get both courage and urbanization.

Alexander the Great (356 - 323 B.C.) partly realized such Aristollian imagination; his Eastern conquests led to the submission of Near East (modern Turkey, Iran, Iraq, Syria, Lebanon, Jordan, Palestine, and Egypt) to Greece, politically and civilizationally. In the era of Romans, Europe did not locate to the North of Asia, but to the West of it. While the Romans made clear the superiority of Europe upon Asia through their success in the warlike deeds, practical arts and laws; the Greeks kept their superiority in the field of science and theoretical arts. Hence the differentiation began between West influenced by Romans and East influenced by Greeks. ${ }^{3}$

Thus, the differentiation with the ancient historical significance for the Europeans, does not describe exactly the development of spatial-relational dimension of the later civilizations; the geographical area of Arabic-Islamic civilization, for example, has extended to Spain in the European Western South, and the term «West» is sometimes used widely in order to refer to Europe, Near East and Northern Africa, opposite to Far East (China, India and Japan). Even if we disregard the inaccuracy of the word «East» from the spatial perspective, we can not overlook the European division of the general history into «ancient», «medieval», and «modern». This division led to the look at the East as a world that remained isolated from the influence of Greek and Roman civilizations in the ancient times, and led to the denial of the influence of its civilization upon the modern European one. ${ }^{4}$ In fact, this division does not indicate anything except the conceitedness of European, who regard Europe as the axis of the whole general history of humanity. The evidence, as Spengler (1880 - 1936) and Toynbee rightly noticed, that the mobility from an age to another is truly 
related to an European event. The event by which the history moves from «ancient» to «medieval» is the decline of Rome by Northern Goths tribes, and on the other hand, the closing of four Greek schools (Platonism, Peripatetism, Epicurism, and Stoicism) by the Roman Emperor "Justinian" (482 - 565) as pagan schools. Obviously, it is an European event that does not really relate to the other civilizations of world. Also the event by which the history moves from «medieval» to «modern» is the fall of Constantinople by the Ottomans in 1453, and the decline of the Byzantine Empire. It is an European event that has a great importance for the Europeans in first place, even though it connected with the Muslims to some extent, it is not connected with other civilizations, such as the Chinese and the Indus ones. ${ }^{5}$ Thus, the humanity's evolution is not fairly divided upon the world's main events, but upon Europeans' main events, so that is the evolution of Europeans/Westerners (since Eastern Europeans were ignored), not the World's evolution.

That is all about the use of the term «East» geographically and historically; we saw that it expresses a pure European point of view, and so its understanding and acceptance depends on the spatial-temporal context of its users. Yet there is another disagreement among Arabs and / or Muslims about the designation of their civilization; it is a disagreement that the builders themselves of this civilization did not know about it despite their numerous differences, but it arose among their successors under the influence of their contrastive cultural attitudes and respondencies, and some orientalists waded through it.

Thus, while some researchers go to designate the intellectual legacy written in Arabic language in the name of "Arabic civilization», others see the appropriate name of it the «Islamic civilization». It is normal to find many arguments with each team to support its point of view, but we must note that some of these arguments have no objective motive. On the contrary, they were and still are a mere reaction, intended 
or not, to the challenge of modern Western civilization, that the Islamic world was divided into two distinct cultural groups; the first took the appearance of herodianism, ${ }^{6}$ or rather the pseudo-morphosis, ${ }^{7}$ in the cloak of Western civilization. Next it turned to erase the features of Islamic identity in the memory of contemporary Arabic mind, and that is the secularism, ${ }^{8}$ which received a strong support from many of the regimes loyal politically and economically to the West, without the slightest benefit from the modern Turkish experience!. ${ }^{9}$ The second group moved in the opposite direction, where it adopted the appearance of zealotism, ${ }^{10}$ or the self-absorption, satisfying with the ancestry's achievements, and took the religion, formally and objectively, a cuirass in front of the cultural attack. It seemed as a jump to the back, or as petrified civilizational sediment with respect to the vital energy. In fact, both of groups seek to escape from the incubus of reality, but only by passing the factor of time, with the constancy of factor of space. The error lies in them, and the most important cause of the acute collision between them is their concept of the big gap between $<A>$, and $<$ Anti$A>$, without looking at the series of neutrality extending between them, which is expressed by the Quran verse:

Thus we made an Umma (nation) of you Muslims with balanced reason so that you be witness of people actions characterizing their rival system

$($ Al-Baqarah $=$ The Heifer, verse 143)

The most noticeable paradox in the Arabic world today may appear when you want to be famous. Then you must follow one of two paths; either you attack the religion and impugn some of its constants, or you tickle the feelings of the silent majority, engaging in the call to religion and in formal legal opinion, even if in an attractive modern costume that hide the emptiness of educational structure of the Shara's intentions and sciences (Shara or Shariah = Islamic law). For the third of two paths, you may not find any trace of it, and even though 
anyone was found, the one who takes part must bear the distrusts of leaders of the two contradictory paths.

Let us demonstrate the arguments of the two collided groups in respect to the problem of designation, and we shall begin with the mental life of Arabs before Islam.

\section{A. Was there an Arabic Civilization before Islam?}

The first reason of the disagreement between those who advocate the name «Arabic civilization» and those who support the name «Islamic civilization», is the type of mental life of Arabs before Islam: did they have intellectual bases that allow them to build a civilization regardless of what Islam offered?. Although this question ignores the normal stages of development for any civilization, but it took a lot of historical studies which were carried out by a lot of Arabs and orientalists for varied purposes. Firstly, let us have a look at some examples of those who deny the existence of civilization before Islam.

In his Islam is a movable global power, the German orientalist Herbert Gottschalk states: «Before Islam, the Arabic area had no significant role in the record of world history. It only occupied a simple marginal place in this history, because the life in desert was a nomadic occasional life, so that there were no common utilities among the tribes, on which the state might emerge. Even the religious liturgies in Mecca, where they went for pilgrimage (Hajj), did not gather them on one way or direct them to a common goal. So the life was confused and unstable. Its general appearance is staying and traveling; conflict and combat»». ${ }^{11}$

In his Some of the treasures of Islam, Dr. Muhammad Ghallab states: «Some European researchers designate the Islamic intellectual fruits the name of Arabic legacy. We disagree with them about this designation, because we see that these mental fruits are a born of Islam, not a born of pure Arabic thought. To verify our opinion, we make two views: one on the Arabic nation before Islam, and other after it, we 
will find that this nation in the first case sterile and waste, while it is fertile and bright in the second case. If these ideas were Arabic, not Islamic, we must have found their first elements in the Arabic environments before the brilliance of Quran in them». ${ }^{12}$

Ibn Khaldun's opinion (1332 - 1406) maybe the most widespread opinion, which he presented in many places of his Introduction (Al-Muqaddimah), its summary states that the Arab is «barbarous, plunderer, and spoiler. If he dominates any regality, the destruction hurries to it. He doesn't submit to a chief easily, isn't skilled in a craft, doesn't master a science, and doesn't have a predisposition to be proficient in either of them. Yet he is in a sound state of temper, prepared to the good, and courageous». ${ }^{13}$

As for the representatives of the opposite view, for example, the French orientalist Gustave Le Bon (1841 1931), who wrote in his «The civilization of Arabs»: «It is impossible that a civilization and language of a nation to appear on the stage of history except as a consequence of slow maturity. Persons, nations, systems, and doctrines only develop gradually. Arabs, who could build a state and create a new worldwide civilization in less than a century, undoubtedly have the talents that can not be completed but for the succession of heredity and by a previous continuous culture. With the help of Arabs, Muhammad could build those shining cities which remain for eight centuries as centers for science, literature and arts in both Asia and Europe». ${ }^{14}$

On the same loom, Adib Lahud weaves in his book The civilization of Arabs before and after Islam, where he states: «Some people claim that Arabs before Islam were in an anarchy and ignorance state, haven't got any work except invading, spoliation and war in the steppes of Najd and al-Hijaz (in Arabian Peninsula). But this claim appears invalid when we see the Arabic nation in its first jahiliyyah (its status before Islam) as one of the most distinguished nations in 
civilization, and its language is the most superior one in the world in its manners, meanings, or syntax ».

In fact, these two opposite opinions aren't away from extremism that kills the neutrality; the first dispossess them of any feature of civilization before Islam, and the second ascribes these features to them, which were not available but to a civilization in its phase of power and prosperity. Both of the opinions, as we mentioned, ignore the natural stages of development of any civilization; i.e., the phase of childhood, the phase of maturity, and the phase of senility. The Arabs, like any people have a civilization, passed the phase of childhood, in which it was not demanded to present any civilizational creations that go beyond their capabilities, otherwise we would ask, for example, about the civilizational features of the ancient Egyptians before their Pharaonic creations, or about the civilizational features of the Greeks before the building of their philosophical and scientific systems. Even 'Ibn Khaldun', regardless of his exactness in research, and despite the genuineness of his theory in the periodical succession of civilizations, didn't exactly define what he meant with the word 'Arab' and in any phase, but he mixed in his judgment among the different ages of the Arab, and released a general judgment on him, although he himself said that the Arabs change according to the environment. ${ }^{16}$

It's true that the Arabs had phenomena of promising mental life before Islam, and we can see them in the language, poetry, aphorisms and novels, as well as they had knowledge in the descents, sky and surging, and some of medicine, yet, the manifest error is the designation of these things 'Science' or 'Philosophy', in the meaning that we find them after Islam, or in the meaning that we can compare between science or philosophy of the Arabs, and science or philosophy of the other people in world.

In other words, the scientific research requires a methodical mind with an ability of organization, proving, and refutation, and it requires daily problems that motivate the tendency of 
research and the desire to tame the reality. These are factors that weren't available to Arabs in their phase of nomadism, but they possessed them by Islam.

We can not then build a border between the two properties 'Arabic' and 'Islamic', but they are, from the historical perspective, one thing: 'The Arabic-Islamic civilization'. It's a unit that refuses disorder or separation.

\section{B. Islamic Culture and Arabic Language}

The second dimension of disagreement about the designation is a spatial - linguistic one, as it is clear in the dialectic answers that introduced by the researchers to the two following related questions.

1. Were the Arabs, as a nationality with borders, the sole builders of that civilization? If the answer is 'yes', then it is an Arabic civilization, even if the Islam was its basis. Meanwhile, if the answer is 'no', then it is an Islamic civilization, the Arabs participated in with a role, significant or not, as well as the Non-Arabs.

2. What about the linguistic structure of the intellectual heritage of Islam?. Isn't it mostly an Arabic structure and we know that this language is the language of Quran, which is the first source of legislation and the support of civilization? Again, if the answer is 'yes', then it is an Arabic civilization, but if the answer is 'no', then it is necessarily an Islamic one, doesn't acknowledge the nationalities.

There are other questions can be answered by 'yes' and 'no' in the same time. This means, as we shall see, that both Arabic civilization and Islamic civilization have common and noncommon parts simultaneously.

This discordant dimension stimulated some great orientalists, such as the French 'Henri Corbin' (1903-1978), the Hungarian 'I. Goldzher' (1850 - 1921), and the British 'E. G. Brown' (1862 - 1929). It also stimulated a significant number of Arab researchers, as some preferred to the use of 
the name 'Islamic civilization', arguing that many who have contributed to building this civilization were not Arabs, but they met under the banner of Islam. At the same time, others preferred the use of the name 'Arabic civilization', because most of the intellectual legacy of the civilization (philosophical and scientific) is written in Arabic language, and because the language, not the religion, what gives the intellectual result its identity. ${ }^{17}$ Let us give some examples.

In his book The Emergence of Philosophical Thought in Islam, Dr. Ali Sami Al-Nashar states: «Some of philosophy historians spoke about an Arabic philosophy, but, we rather speak about Islamic philosophy, otherwise we'll bring out the majority of Muslim thinkers from the field of our research, because most of them were not Arabs. Although a lot Arabs played a great role in the history of Islamic thought, the Persian thinkers, who embraced Islam rightly and certainly, also played a great and important role in the construction and supporting of this thought». ${ }^{18}$

In spite of the fact that 'I. Brown' has a book titled 'Arabic medicine', he announces in the same book that he prefers the term 'Islamic civilization' to the term 'Arabic civilization'. He justifies that in his saying:

"As the Latin language was the language of science in Europe in the medieval ages, the Arabic language was the language of science in the whole of Islamic world. There is no objection to the speech about 'Arabic science', or 'Arabic medicine' if we regard that this speech only means the set of scientific or medical principles that were written in Arabic language, because we didn't begin the comparison between what we can call scientific books, which are written by Arabs themselves, and ones which were written in Arabic language by others from Islamic - non Arabic countries, except since the eleventh century. Most of these books were written by Persians, Syrians and Jews, a few of them were written by Greeks, yet, the pure Arabs didn't write but a bit,..., even the religious sciences [The interpretation of Quran, Hadith (the 
Prophetic tradition), legislation, etc.], the Arabic part in them, as 'Goldzher' referred, was underdeveloped as for the nonArabic one». ${ }^{19}$

Also 'Bartold', who firstly stressed on the power and attraction of Arabic language, then wrote:

«In the seventh and eighth centuries, the Arabs inserted under their authority a lot of people, who were clearly superior to them in the civilization, but the Arabs didn't lose their nationality as the Germanus in Europe did, or the Mughals in Asia,..., We can interpret such currency of Arabic language as the Arabs didn't depend on the power of weapon only, such as the Ancient Germanus, Mughals and Persians, but they established, since the seventh century, an advanced literary language». ${ }^{20}$

Nevertheless, 'Bartold' goes back to stress that the Arabs have been influenced by the other languages of peoples in the countries that they captured. To prove so, we find: «That the golden coin of Muslims was called 'Dinar' (from the Latin word 'Denarius'), and the silver coin was called 'Dirham' (from the Greek word 'Drachma'), and the envoys who conveyed the messages of government were called 'Bareed' (Mail), it is a word taken from the Latin one 'Weredus'». ${ }^{21}$ Moreover, as 'Bartold' refers, Kufa and Basra were two active centers of the scientific life, where there was no city, in the first century A. H. (the seventh century A. D.), that could rival them. In them, the foreigners (Non-Arabs) and their students, who embraced Islam, compiled the sciences of doctrines and jurisprudence, and then it was established a school (a doctrine) of the grammarians and linguists in both cities. However, most of those, who compiled the Arabic sciences, were not Arabs, but foreigners». ${ }^{22}$

No doubt that these sayings exaggerate in minimizing the role of Arabs in the formation of civilization, which is not certain by the history of Arabic and Islamic sciences. However, the leaders of grammarians were from Arabs, and the Persian philologist 'Sibawayh' $(750-795)$ was not but a 
student of the Arabian lexicographer 'al-Khalil Ibn Ahmed alFarahidi' $(718$ - 791).

The Arabs contributed in the sciences of doctrines and jurisprudence with a great role which is not less than the role of Non -Arabs. It's fair enough to say that the whole laborious leaders, except 'Abu-Hanifa' (696-767), were from Arabs. ${ }^{23}$

There is parallel to an observable exaggeration - without an acceptable justification, in the evaluation of Arabic language's role as an effective element in the rise of civilization; either through connecting it with the religion as a language of the Quran, or by the direct connection between it and intellectual creation of those who were skilled in speaking it. If that is true, then the whole Arabs must be creators, and the Arabs must be preferable to non Arabs because they speak the holy language. Yet, the facts of history don't support this matter, and it is what Islam denied intensely by the Messenger when he said: «There is no superiority for Arab to Non Arab, nor for Non Arab to Arab, nor for white to black, nor for black to white, but by the piety. People are from Adam, and Adam is from earth».

The Islamic state extended its influence over vast lands that cover great parts of Asia, Africa and Europe some peoples of these states adopted Arabic language, such as Egypt, Cham, Iraq and the states of Arabic Occident (Maghreb), but the majority of peoples, who embraced Islam, still hold on to their native language. The Arab conquerors didn't think of the imposition of Arabic language on peoples. That is why the majority of Muslims don't speak Arabic. According to the latest estimations, there are today 1.25 billion Muslims in the world, knowing that there are no more than 240 million people whose mother language is Arabic, including more than ten million non Muslims. The percentage of Muslims, whose mother language, is Arabic, about 19.2 percent of the total of Muslims in the world. With a simple calculation, there are 82 percent of Muslims who don't speak Arabic, which we - the Arabs - regard the basic corner of the religion! $!^{24}$ 
On the totally, the previous arguments lead to a consequence that the circle of Arabism doesn't correspond to the circle of Islam. That is extremely true as among the Arabs, there were Jews, Christians, Sabians and Majus (fireworshippers). Similarly, among the Muslims, there were Turks, Ajams, Persians and others from the Non-Arabic races. Yet, when we speak about the civilizational dimension, we must not forget that the two circles are overlapped to a great degree. The civilization which we talk about is an Arabic one, because the books left behind are written in Arabic language. It is also an Islamic one, because those who wrote books lived in the home of Islam: they lived under the auspices of it, and were satisfied with its concept. Islam added to them, and they didn't lag behind from supporting with their cultural traditions, Arabs and Non-Arabs.

\section{Is It a Theological Civilization?}

The ultimate point that remains with respect to the problem of designation is the question about the religious nature of Islamic civilization. The supporters of the use the name 'Arabic civilization' argue that the word 'Islamic' inspires that we are up to a theological civilization'; its stature is the religion, and its motives are the creeds and problems of that religion. Thus, it is not a specific human civilization, which can contain the whole of human's interests in a specific space and time. This understanding, of course, can stoke up the hell of religious intolerance, and open the door for the irrational comparisons among 'Islamic mind', 'Jewish mind', 'Christian mind', 'Boddhist mind', ... etc., especially that we live in an era, in which the media play a very serious role in the formation and directing of the public opinion from one dimensional view that is not prepared to accept the other. ${ }^{25}$ Moreover, the use of the word 'Islamic', from the same perspective, may not be in the favour of Muslims. That appears when we compare the 'Islamic civilization' with the modern European renaissance, and how the later didn't realize 
but for the European thought became free from the domination of church's men over the whole of aspects of intellectual life. We still remember the phrase of 'Galileo' $(1546$ - 1642) when he was judged as a criminal by the church, because he adopted the astronomical system of 'Copernicus' (1473 - 1543). Under threat of torture he was forced to write: "I'm Galileo Galilei, by my hand, announce the renegading from my doctrines". We still remember too the story of Giordano Bruno (1584 1600), who was kept in a dark dungeon for eight years and then taken out to a blazing market place and roasted to death by fire! ${ }^{26}$

But this debate, from the opposite view, is repulsed from two aspects; the first one, if the word 'Islamic' attributes to the civilization a theological feature that is loathsome by the West due to the past, then the word 'Arabic' also attributes to the civilization a racial feature that is high loathsome by everyone. Where the speech goes on about Arabic mind, another English, a third German a forth French, ... etc., which reminds us of the eugenics movement and its unethical practices during the first quarter of twentieth century. ${ }^{27}$ If Islam, as we mentioned, has forbidden any racial discrimination among humans, the racism has a long history, undesired, in the memory of West. It is a history that still prevails in many biological, historical and anthropological researches, specially with respect to the rise and decline of civilizations. ${ }^{28}$ On the other hand, we can not remove the theological feature from the Arabic civilization easily, because the religion was an inexorable shaping force of this civilization, and the philosophical and scientific creation was a direct response to its open call to the reflection and use the mind with regard to the affairs of life and its requirements. Thus, if we look at the topics of Islamic philosophy, as 'Dr. Ahmed Mahmoud Subhy' notices, we will find that it is confusedly, but mistakenly, to name it 'Arabic philosophy'; because there is no thing in them that relates to the nationality or race in order to attribute them to the Arabism or Arabic, but all of them 
focus on 'religion' and links up with it in some aspect; there are 'the scholastic theology' (Ilm al-Kalam), which is correspond to the theology in Christianity; 'the philosophers of Islam', whose purpose is the reconciliation between philosophy and religion, not between philosophy and nationality; 'mysticism', where there are Boddhist, Christian and Jewish mysticism, but there are no English or French mysticism in order to say Arabic mysticism or Arabic philosophy. We can understand the whole of these topics regardless of the 'Arabic' as a nationality or language, but we can not understand them in any case regardless the religion: Islam. $^{29}$

The same thing as to science; Astronomy and Trigonometry, for example, were very close connected with three of the five bases of Islam: Prayer (Salat), Fast (Sawm) and Pilgrimage (Hajj); also Arithmãtĩqĩ was close connected with the calculation of heritages, ${ }^{30} \ldots$ etc. However, there is no sense of the comparison with the modern European renaissance, after the Western thought became free from the domination of Popes and church's men, because Islamic state didn't know the courts of inspection or the documents of forgiveness! .

In this matter, Dr. A. I. Sabra reports two important observations that prevent the comparison; the first one states: As far as science and philosophy are concerned, the European Renaissance of the sixteenth century was in part a reaction, which became more pronounced in the seventeenth century, against patterns of thought and argument associated with medieval 'scholasticism'. In Islamic history, events followed the reverse order: the "renaissance" (if that is the right word) came first, in the ninth and tenth centuries, and a form of scholasticism followed, though not immediately and not uniformly in all parts of the Muslim world. The second observation points to another contrast between Islam and medieval Europe. In Islam, whether in ninth - and tenth century Baghdad, eleven - century Egypt and central Asia, 
twelfth - century Spain, thirteenth - century Maragha in Iran, or fifteenth - century Samarkand, the major scientific work associated with the name of those who were active at those times and places was carried out under the patronage of rules whose primary interests lay in the practical benefits promised by the practitioners of Medicine, Astronomy, Astrology and Applied Mathematics. Many of those practitioners were also prolific writers on "philosophy", a mode of thinking known by the Arabicized term "falsafa" and characterized to a large extent by a mixture of Aristotelian and Neoplatonic doctrines and forms of argument - the kind of mixture we find, for example, in the works of Al-Kindi (796 - 873), Al-Farabi, and Avicenna. In those circumstances science and "philosophy", or falsafa, were secular activities that were practiced, developed, and propagated as rational inquiries independent of any religious authority - which, of course, did not prevent the proponents of this autonomous, self - legitimizing mode of thinking from offering their own rationalistic (i.e., Hellenic) interpretations of religious doctrines such as revelation or prophecy or providence and of religious institutions such as law. After all, falsafa was an all-embracing world view that claimed the right to scrutinize and account for everything within the sphere of human experience, including religious experience. In one case among the prominent devotees of falsafa, that of Al-Kindi, a serious compromise was made by renouncing the Greek doctrine of the eternity of the world in favor of 'creatio ex nihilo'. But unlike most of their Christian counterparts in medieval Europe, Islamic philosophers (the self-styled falasifa) and philosopher - scientists in that Greek sense were not "theologians" or members of religious organizations. The one major exception, of sorts, is the twelfth - century Andalusian Averroes (1126 - 1198), who came from a celebrated traditional family of Malikite jurists and practiced the Malikite version of Muslim law as a judge, but who, nevertheless, believed himself to have inherited the mantle of Aristotle. $^{31}$ 
Then, there is no place of comparison between an 'Islamic' renaissance or civilization, whose motive is the religion, and another 'European', whose motive is the marginization of religion. Both of them have a different local climate. However, if the Muslim civilization has declined and vanished, the reason was not Islam as a religion, or the intolerance of jurists lurked to destroy any civilizational achievement, but there are many factors that are not related to the religion, noticeably the dictatorship of governors, the internal political corruption and the external crushing raids of Mughals which accompanied it. Besides the discovery of 'the cape of good hope', where the Muslim state lost its resources, we will come back to that later.

What we would like to say that the two characters "Arabic" and "Islamic" are equivalent indicatively if we put into consideration the Western perspective invoked by some, and they are also equivalent if we take into consideration the religious and civilizational perspective invoked by others. Thus the two contrasts meet and integrate in a single neutrosophic system; Arabism is Islamic in the culture, tendency; and Islam is Arabic in the origin, spirit, and language.

In the end, we must assert that the sciences, philosophy, art, and all semblances of civilization, are nothing but free innovations of the pure reason, representing links in an infinite series of the accumulative human effort in general. Hence, they represent common property of all humankind, whatever its nationality or religion, and God is truth when he says:

\section{As to the forth and the scum, they are rejected and disappear, where as what is profitable to the people remains behind on earth}

$\left(A l-R^{\prime} a d=\right.$ The Thunder, verse 17$)$ 


\subsection{Potentials: the Positivity of Negation and the Richness of Difference}

With the potentials we mean here those foundations and factors, on which the civilization depends in its genesis and evolution that make it continuous in space and time in accordance to a general framework that represents its identity. In case they are absent, the civilization will demise and degenerate. Whatever these potentials that are necessarily different from a local dimension to another, there is a condition must be fulfilled so that the civilization can go upright on sound bases. It is the eradication of errors that shackled the society before and prevented it from waking up of its slumber and to blockade these errors, if possible, so as not to re-emerge. «It is futile to fill the vessel of truth in our minds, when the vessel of falsehood, still persists in the neighborhood of it; the falsehood, over time, is meriting to spoil the truth, as the corrupt fruit spoils the sound fruits around it ; if the truth comes and emerges, by any means, the falsehood must also perish at the same moment ; the setting up of right and the correcting of wrong are two aspects of one attitude: i.e., the affirmation and negation, which go to gather, so that the first proves what is worthy of proof, and the second erases what must be erased»..

This is the first step to the rise of any civilization, according to which the unity of human nature realizes in its clear form. But that does not mean at the same time the difference of peoples in respect to the mental and psychological composition of personality; the personality of ancient Egyptians unlike the personality of Europeans, and both of them unlike the personality of Arabs. However, the difference is not due to genetic - hereditary reasons, but to ones imposed by the natural and social environment. In other words, we can say that the method leading to the rise civilization is one method; the method of distinction of right from wrong, and 
truth from falsehood. Yet, the source of this method, its elements, and its circumstances of application vary from one nation to another and from one era to another. The source may for example, be the religion inspired by heaven, or the mind with its logical judgments and inferences, or it may combine the two in one integrated system. That's from a point of view and, on the other, what is right in accordance with certain conditions, may occur in the circle of error in accordance to other conditions, and what is true at all in a specific time and space, is just on the contrary in other time and space. There is a continuous series of the degrees of truth between them. That is what we call the relative truth, where we find, on the whole, that the same argument is true in some worlds, but false in other worlds; even ' $1+1=2$ ' is true only in the decimal system because in, for example, binary system of numeration $' 1+1=10$ '. As for the absolute truth, where we find the same argument is true in all possible worlds, it is confined only to God, according to Islamic belief.

If we look at Arabic - Islamic civilization from this perspective, we'll find that across the stages of its development, it based on three basic potentials. They are: A) The revelation of heaven (i.e., Quran and Sunnah, they are the main source of method. It is a method able to avoid the pitfalls of way with its flexibility and ability to contain the variables). B) The external conquests (by which the process of cross - pollination between Arabic - Islamic culture and other cultures started, and the picture of mind that is capable of creativity completed). C) The internal structure (on which the balance of neutrosophic equilibrium of civilization settled, despite the wide spread of conflicting ideas in its various aspects). Namely, when an idea $<\mathrm{A}>$ appear, not only its opposite $<$ anti-A $>$ but also neutral ideas arise $<$ neut-A $>$; but these three categories are dynamic and they continuously change. 


\section{A. The Revelation of Heaven (Quran and Sunnah)}

Islam came at a time when the life of Arabs did not differ greatly from what it is today. There were exaggeration in individual freedoms, immersion in materials, loss of the future view, absence of the entire comprehensive vision, inability to link the causes with its results, a courage motivated by the nervous mood and the sanctity of dignity, and eloquence in the speech does not exceed the glibness and the beauty of rhetoric, ...etc.

Islam had two effects on the Arab mentality from two different sides: The first is direct, namely its legislations and tenets that were brought opposite to the beliefs of Arabs, and they served as the tool of screening, negation, proof and construction of virtues, and virtues misted up by sins, which life is filled with. The second side is indirect, as Islam with its new method enabled the Arabs to conquer Persia and the Roman colonies which were two great nations with the highest civilization at this era. It was the impact of conquest of these two nations, with what they enjoyed of different social, scientific, and philosophical systems that their civilization leaked out to Muslim, whose minds were influenced by them. $^{34}$

As for the legislations of Islam and its tents, they are contained in the Quran and Sunnah as a way's method precluding the deceptions of way and rectifying the passions of soul (Shariah). They are, as we will see, two complementary sections of a religion that balance the needs of spirit and the demands of body.

\section{Quran:}

Quran is the speech of Allah, descendant to his Messenger, Muhammad (God's praise and peace upon him), and written in the Quran books. The word 'Quran' in terms of the verbal derivation refers to the Arabic triple verb: 'qara'a' (to read or to recite), which is used in Arabic language in two meanings: 'read the book', i.e., peruse its words by seeing, with or 
without uttering in speech and 'read the thing', i.e., collect and adjoin it. Hence, Quran linguistically is a name of a group of sheets, attached together for the purpose of speculation, contemplation and guidance. ${ }^{35}$ There is a clear correlation between the first verse of Quran, chronologically, and the name 'Quran'; the verse begins with the divine command 'Read': «Read in the name of Allah your Creator» (Al-Alaq $=$ The clinging organism, verse 1 )

Then, Quran is a divine comprehensive invitation to reading, learning and dust the ignorance.

In terms of it is a guided method, Quran included regulatory judgments that cover all the concerns of man. It is the kind expressed by God:

... Indeed there came to you intellectual illumination and enlightenment and a book standing manifest of glory, knowledge and spiritual light. A book, the spirit of truth guiding those who lift to Allah their in ward sight into all truth, leading them to the path of safety and peace and guiding them, Allah willing, out of darkness and superstition of later times and out of want of spiritual and intellectual sight into intellectual illumination and enlightenment and directing them to the path of righteousness

$($ Al-Maidah $=$ Heaven's sent festive table, verses 15, 16)

In terms of it as a divine being, its judgments are established and they have the ability to the conditions and the changing social influences; namely, that it addresses that man in charge at all times and places.

If we look to the judgments of Quran and its legislations, we see that they are divided into three sections: ${ }^{36}$

- Judgments relating to the doctrine, such as the faith in God, His Angels, Books, Messengers and the Day of Resurrection. These are the believable judgments, which are studied under the science of Monotheism. 
- Judgments concerning the redress and refining of the soul. These are the moral judgments, which are studied in Ethics or Mysticism.

- Practical judgments that relate to the sayings and deeds of charges, i.e., the jurisprudential judgment. They are of two types:

Type one: The judgments of worships, such as the prayer and fast. The purpose of them is the organization of relationship of the individual with his God.

Type two: The judgments of transactions, such as the judgments of family relationships, the financial transactions, the judiciary, the crimes, the regime of government, the international relations, the economic systems, and other matters that occur within the scope of private and public laws, according to the modern legal terminology. The purpose of these judgments is the organization of relationship of the individual with another individual, or the individual with the group, or the group with another group.

The Arabs profited from these judgments from three sides; firstly, they increased the mental level of Arabs to a degree that made them move from a situation to its contrast, doctrinally, morally and socially. After the Arabs were worshippers of idols and images, required to consider the degradation of speculation and the degeneration of thought, they worshipped God that goes up upon the material embodiment, as the Quran-ahu says:

He is not apprehended by sight whereas He comprehends all that is displayed to the bodily eye and all that is brought before the mind's eye, and $\mathrm{He}$ is the Gracious Who gives His servants an understanding heart, and $\mathrm{He}$ is Al-Latif (Kind) and Al-Khabir

$$
(\text { Al-Anaam }=\text { Cattle, verse 103) }
$$

Also after the God for most of them was a tribe's God, just as the case for the Jews before, they knew that $\mathrm{He}$ is a sole God for all peoples, and that the message of heaven has been 
completed with their Book and Prophet: the last completeness. Moreover, they knew that they bear the burden of the spread of religion and the call to its teachings. That was the source point of them to the outside world and the mixing with the other nations.

Quran also has had a significant impact in changing the value of things, ethics and social relations in the eyes of Arabs, so that the value of some things has risen, while the value of others has fallen, and the conditions of life in their view became different from yesterdays. I do not find any expression of the difference between the two cases better than indicated by 'Jafar Ibn Abi Talib', who was one of those who migrated to Abyssinia (Habesha). He said to 'Negus', when the later asked him for state: «We were a people of paganism: worshipping the idols, eating the flesh of a dead beast, doing the obscenes, uncommunicating the relatives, offending the neighbourhood, and the strong from us was eating the weak. We used to do so until God sent us our Messenger, whom we know his origin, sincerity, honesty and modesty; He called us to the monotheism and worship God, and to leave the stones and idols that we, and our parents, were worshipping. He also commanded us to speak the truth, reimburse the deposit, keep who are related to us by blood in touch, give charity to the neighbour, and stop the incest and the bloodshed. He also prohibited us from the obscenes, telling a falsehood as well as the intentional assertion of what is false, wrongfully eat the orphan out of his property, defamation the virtuous women who are unaware of what is traduced in propagation. He commanded us to worship Allah and do not incorporate with Him other deities, and commanded us to perform the prayer, fast, and give the alms (Zakat). Then, we believed and have faith in him. But that made our people show enmity toward us, they tortured and seduced us in order to return to worship idols instead of the worship of Allah, and to legalize the wickedness that we legalized before. So, when they defeated 
and oppressed us; confined and prevented us from our religion, we came up to your homes».

Despite the doubts that this story is invented, because the fast is mentioned in it while it was not legislated but after the migration to Abyssinia, it represents the conflict between the two mentalities: the Paganic and the Islamic, the most honest representation. $^{37}$

The second side of profitability is shown in the tendency of philosophical and scientific research, which Quran diffused in the spirits of Arabs; Quran portrayed for them the idea of deification in its latest from (Metaphysics), and pushed them to extrapolate the appearances and laws of nature (physics): Quran announced 'the unity of Allah and His effectiveness', in the opposite of each Greek conception about the idea of God, thus denied any Greek conception of the idea of God, whether He is a Demiurage or le premier moteur or Moteur immobile, ... etc. Quran also announced the idea of creation: "Allah is the creator; He created everything from none; He created the world from nihility", then Quran denied the idea of eternity of matter. As it announced the beginning of time, it also announced that the time has an end, and then denied the everlasting and the endless of matter. As long as Quran has denied the eternity of matter, it has announced the contingency of world, then turns with the mind from metaphysics to physics; i.e., to the research in the appearances of nature and its laws: its constancy, changes, certainty, possibility, ... etc.

Through the mind, the man appears: the successor of Allah on earth, and the greatest example of the unlimited power of Allah; On the top of universe, Adam is created by hands of Allah; Allah made him a mix of Angels and animals, and raised him over Angels, then put both virtue and sin in him, dropped him to the vast universe in order to build and sabotage, construct and destroy. Then Allah began to evolve the human's mind and body, but the evolution here is only in the scope of human species, not in another scope, thus Adam (or human) moved from a form to another and from phase to 
phase, and the messages of Allah follow in succession until the evolution has been completed with Quran, and the evolved humanity became stable, but it rushed to the scope of experience and science, as required by Quran.

Quran also tended to man to glorify him in his latest phase, and push him to explore the cosmic horizons, regarded him responsible for any of his actions, and for any of his clams:

Here comes the correction of the wrong belief held by those who thought they would not be rewarded for what they give if it be too little, and he who has done an atom's weight of evil, will see it and be punished for it

$($ Al-Zalzalah $=$ The earth in convulsions, verses 7,8)

Then he put the individual responsibility, which is the feature of modern life, in the strongest manner. Quran denied «the crucifixion of the Christ» as a redemption of the group, and saw that there is no thing can redeem the human except the work of human himself, not the sacrifice from a Prophet or Messenger. It left the human goes between the good and the evil, but the acquisition of any of them is by his hands, and Allah is aware of both of them, so He put the reward and the punishment in another world, which is a secret for us. ${ }^{38}$

As the third side of profitability from the judgments and teachings of Quran, that they taught the Arabs and Muslims the logic of mental difference, the way of exploration of distance between zero and one, and the significance of they mixing and gobbling to each other.

Sahaba (the Prophet's companions) were the most efficient people to understand the Quran in general, because it is descendant in their own language, and they saw the conditions of Revelation. Nevertheless, they differed in understanding depending on their difference in the instruments of understanding. That is because: ${ }^{39}$

- They knew Arabic language with disparity among them, despite Arabic was their mother native language. Some of them knew a lot of the pre Islamic literature, knew its 
equivocals, and seek the help of that to understand the vocabulary of Quran. Others lacked this experience.

- Some of them also were accompanying the Prophet and staying near Him, then watching the reasons of the revelation of verses, but others were not; we know that the knowledge of these reasons is the biggest help to understand the meaning of verses, and the ignorance of them must leads to the error.

- There was also disagreement among them to know the habits of the Arabs in their sayings and deeds. For example, who knew the habits of Arabs in pilgrimage before Islam, can understand the verses of pilgrimage more than who did not know. As well as the verses that are mentioned to criticize the deities of Arabs and their method to worship them, since the understanding of these verses is not completed except those who knew what the Arabs were doing and so on.

- Similar to this is the knowledge about what the Jews and Christians were doing in the Arab Peninsula at the time of the revelation of verses, wherein the reference to their deeds and refutation for them. This is not understood but by means of knowing what they were doing. Because that and the like, it was the difference among Sahaba in understanding, and for the followers and who came after them, as we shall see, the most different.

\section{Sunnah:}

Sunnah has a meaning in language, a meaning in the idiom of jurists, and a meaning for the fundamentalists. In language, Sunnah is the usual way that is preserved, and under it the act reoccurs. An example of that is Allah's saying:

\section{A mode of action Allah has long pursued in dealing with} those who came to this world before you and you shall not find a change in His mode of action

$(A l-A h z a b=$ The confederates, verse 62) 
The man's Sunnah is his way that he complies with and maintains, whether for what is praiseworthy or what is dispraiseworthy.

In the terminology of jurists, Sunnah is what was superfluous worship transferred from the Prophet ..., which is not duty. The word may also be used by others to means what is opposite the «heresy»; hence we say 'so and so has a Sunnah', if he worked according to the Prophet's work, and 'so and so has a heresy' if he worked otherwise.

For the fundamentalists, Sunnah is what was said, done and approved by the Prophet other than Quran. The approval is what He confirmed of the deeds of Sahaba by the silence with an indication of complacency, or by showing the approval and support. In this sense, Sunnah is regarded as one of the evidences of judgments, and one of the sources of legislation. ${ }^{40}$

By Sunnah, the method of Islam becomes complete, and the supply of way becomes available, in the evidence of God's saying:

\section{Nor does he give utterance to words, moved by selfish motives, or utters error against Allah and commits its heart to inequity}

$$
\text { (Al-Najm }=\text { The star, verse 3) }
$$

Also Sunnah is what the Muslims are ordered to follow in many verses of Quran in order to complete the faith; ${ }^{41}$ it realizes the meaning of Muhammad's prophecy and the acceptance of his message.

For these reasons, the Prophetic traditions (Hadith) occupied the second place - after Quran - as a source of Islamic law. That is why Muslims specified Hadith with an independent science which is the science of hadith's term. Its aim is the purification of the true hadith from the innovated one, and it is a science with elements and rules which the Imams (leaders) of hadith achieved before the scientists and 
philosophers of history, who took to build a method to correct the historical version after that. ${ }^{42}$

Indeed, the most legal judgments in Islam come back to Sunnah, because a lot of the Quranic verses came generalized or problematic or common, then the detail, clarification, and restriction of them were in Sunnah. Al-Shatibi (d. 790) stated: «Sunnah refers in its meaning to the Book (Quran). It is a detail of its sum, clarification of its problematic, and extension of its brief, because it is a demonstration of it». ${ }^{43}$ Thus, the judgments of Sunnah could be divided in terms of type as follows:

- Judgments which are consistent with the Quranic ones and assuring them. Some of these judgments: prohibiting of disloyalty of parents, false testimony, and killing oneself, besides stimulating in the importance of seeking to acquire science and knowledge, and so on. ${ }^{44}$

- Judgments that are demonstrating the meanings of Quran and detailing of its sum, including the Sunnah that showed the ritual acts of Hajj, the quorum of Zakat and its amount, how much cutting up from the hand of thief, and the times of prayer and its forms, etc. Quran in all that did not deal with the partial details, but its judgments were characterized by generality. The examples of that:

Pay reverence and veneration to $\mathrm{Me}$, and duly engage in worship and give zakat (alms) (in more than one location on the Quranic verses, including: Al-Baqarah = The heifer, verse $43 \&$ Al-Nisa $=$ The women, verse $77 \&$ Physical and Spiritual $=$ Al-Nur, verse 56)

The pilgrimage to there is a duty incumbent on people; those of them who can afford to do homage and honour to Almighty Allah

(Al-Imran $=$ The family of 'Imran', verse 97) 
As to the thief, man or women, who criminally and inexcusably commits a theft - whether by violence or by stealth - you must make them suffer the amputation of their hands in requital of their iniquity

(Al-Maidah = Heaven's sent festive table, verse 38)

The Prophet has explained that in detail with his sayings and deeds.

- Judgments that are restricted the generality of Quran. For example, Quran stated:

These proceedings are concluded after a legacy, he might have bequeathed, has been paid besides a due debt

$$
(\text { Al-Nisa }=\text { The women, verse 11) }
$$

The word 'bequeathed' appeared in the text as absolute. That implicates the permissibility of bequest with any amount, but the Prophet restricted it in his famous hadith for 'Sa'd Ibn Abi Waqqas'. When the latter was ill, he asked the Prophet: «O Messenger of Allah, the anguish has reached me the case that you see, and I have a lot of money, and there is no inheritor for me but a daughter, should I give two thirds of my money as alms? Said: No, I said: the half, O Messenger of Allah?, said: No, I said: the third, O Messenger of Allah?, said: Ok, the third, although the third is a lot ; If you leave your inheritors rich, that is better than you leave them as poor dependent on the people». ${ }^{45}$

- Judgments earmark the general ones of Quran. An example of them is what is mentioned by 'Abd Allah Ibn Mas'ud' about the verse:

Indeed those who believed in Allah whit hearts impressed with the image of religious and spiritual virtues and did not confuse their spiritual apprehension 
of divine truths with wrongful actions and injustice are they who shall escape censure....

$$
(\text { Al-Anaam }=\text { Cattle, verse 82) }
$$

Abd Allah Ibn Mas'ud stated: «when this verse is sent down, this condition was very hard for Muslims, then they said: O Messenger of Allah, who of us does not do injustice to himself?, and He said: it is not so, but it is the polytheism (al-Shirk) ». The Prophet inferred that from the verse:

\section{O my son, never incorporate with Allah other deities, for polytheism is a grave offense and an unforgivable sin indeed}

\section{(Luqmqn, verse 31)}

- Judgments about which the Quran did not speak, but they came by Sunnah, such as the prohibition of eating the flesh of native donkeys, horses, mules, every predatory animal with a tusk, and every bird with a claw. Here we find that Sunnah is completely autonomous as for the legislation, depending on the Messenger's saying: «But I had the Quran and the like with it».

Muslims have benefited from these judgments, as they benefited from the judgments of the Quran and its teachings. The trips of Imams and their tours in the countries were in order to ascertain the true hadith and discriminate it from innovated ones, from the major reasons that led to the dissemination of culture in the Islamic world, and to exchange views and disagreement about them.

This as well as the new events and attitudes, which took place after the discontinuity of revelation without explicit text to deal with them in Quran and Sunnah. And the alien cultures that Muslims faced as a result of the vastness of the Islamic world, which led to the originating of consensus (Ijma) and 
effort (Ijtihad) as new two sources of the legislation, and the emergence of different Islamic sects that still exist up today.

\section{B. Conquests: Mixing and Comprehension of the Other}

\section{Between Islam and Muslims}

The Prophet died $(570-632)$, while Islam did not go beyond the Arab Peninsula. However, He had initiated to call the near nations and skirmish them. A few years after his death, the armies of Muslims, specially in the era of caliph 'Omar Ibn al-Khattab', could establish the foundations of an huge empire, then they conquered al-Sham, Egypt, Iraq, and Persia, hence the Islamic state spread during the Umayyad rule $(667$ - 750) and the Abbasid rule $(750-1258)$, to extends from the Atlantic Ocean to beyond the borders of India and Turkmenistan, from the South Sea to Caucasus countries and to the walls of Constantinople.

This unique political deed was not a result of accidental and vague moment passed by the Arab mind that was very proud of its abilities, or a desire to assault and robbery and satisfy the euphoria of superiority and domination, ..., but was the result of a new spirit, and a new rationality, diffused into the Arab body by a new religion that its principles has been completed, and its method became straight forward for those who embraced it, during the life of the missioner of it. God Almighty says:

\section{Today have I complemented for you your religion and} made all grace abound in you. And I have chosen for you Islam as the acceptable system of faith and worship

( Al-Maidah $=$ Heaven's sent festive table, verse 3$)$.

'Omar' has cried when he heard this verse recited by the Messenger of Allah, and has realized that the revelation has ended, and the moment of death of the Prophet became near; i.e., the pens - employed in writing - are raised and the records have dried up. But he also realized that the Prophet has performed His message as full, and Islam became a 
complete religion with its verses and became a given blessing, then its implementation became in the hands of Muslims who endure the burden of life, and its achievement lies on the shoulder of Islamic nation, which inherited this legacy and that message.

In fact, this Omar's glance embodies inclusively a partial answer to the question on the cause of the deterioration of Arab - Islamic civilization, and the failure of Muslims to catch up with the progress of civilization in spite of the survival of Islam among them with its same method and judgments themselves. The essence of this answer is that there is a necessary distinction between Islam on one hand, and Muslims on the other; Islam is the valuable religion that we talked about before; but the Muslims are those who embraced Islam in all its faith and applied dimensions; just as the intention of faith may or may not be true, also the application may successful or failing. As far as the degree of faith swings between perfection and deficiency, the degree of application also swings between right and wrong. In other words, Islam is a religion that has initial and fixed rules, principles and teachings, driven by the mind and understanding, and refreshed by humans with his quality and safety of properties. Then they need to be accomplished in the existence, and to be implemented in the life, they must coexist with the individual, group and nation to create their results and reveal their meaning. As Muslims, and many of them do not go beyond, with Islam, the circle of name or formal heredity only, they are people who have the Islamic identity; they are, as human beings, subject to the change and transformation, the agreement and disagreement, the ascending and descending. Then, if we overlooked this separation, and said that Islam is Muslims themselves, and adhered Islam to them without a justifiable reason, then we had linked by ignorance between Islam and what Muslims do in every time and place. That is the view of some Western thinkers when they see Muslims weak, submitted to others, and sluggish, they say that Islam 
carries these qualities, and leads to humiliation and dehumanizing. ${ }^{46}$

Take for example, the behaviour of some Muslims in polygamy, just for satisfying their desires and cravings, or take their behaviour in the use of the right of divorce unconditionally, without prior warning or justification, and without understanding or awareness of the truth of Islam. There is no doubt that the view of non-Muslims to such behaviors is against Islam and its judgments, while Islam is completely innocent of this outrageous charge. God's Holy says - regarding polygamy:

And you may join in wedlock with as many as two, three, or four of other women of your choice, with the proviso of observing Islamic principles. But if you fear you shall not to able to exercise justice among these many, then do not marry more than one, or you may adopt, under the circumstances, the captives on hand into intimate relation of marriage or into intimacy, for the operation of natural instinct or innate propensity that is more sure and simple, than that of reason needs to be satisfied but in the frame of Islamic principles of kindness and equity: This prudent course of action is less likely to make you depart from moral righteousness and not to have children whom you cannot supply with the necessities of life

$$
\text { (Al-Nisa }=\text { The women, verse } 3) \text {. }
$$

Thus, there are prerequisites for allowing polygamy, they are ones in which we find the familial and social protection, and the prevention of falling in what is more evil than polygamy, also the maintaining the morals and health from shame, and the honest care for women.

As for the divorce, the Messenger said: «The most hateful of lawful as to God is the divorce». The divorce in Islam is a human peaceful solution of unavoidable strife. It is to appease the conscience in non-restriction with a life in which there is no excellence or tranquility, and it is to give everyone his right 
in the freedom that not include any form of the injustice. The divorce is not a prerogative of the man, but a judgment in which there is a religious responsibility before God and society. ${ }^{47}$

Take also the political practices of Muslims and their misconception for the idea of installation of the Governor and his relationship to the people, after the age of orthodox caliphs $(632$ - 661), the first thing that you notices is that the uncontrolled tendency by some to the monopoly of authority as if it is a hereditary right which is not governed by the competences and their objectivity, and is not disproved by the rights of people in the checking and choosing, even that is rare, across the entire history of Arabs and Muslims, that you find a governor or official has left his position voluntarily; the governor remains in his office as long as he is alive or strong with his clique, and the government remains as long as the governor satisfied with it, and as long as it performs the conditions of loyalty and obedience to him during the night and at both ends of the day!.

The second thing that you notice in the political life of Muslims, as a direct result of the first appearance, is that the exaggerated venerations of the personality of governor and his ideas, or of the official and his directions in any position, who has a political power, has at the same time, because of his political power, 'the opinion', not has an opinion (without the definite article), so his own opinion does not prevent the others have their own opinions. ${ }^{48}$ The governor in the culture of Arabs and Muslims is the hero who makes the history. If we want a more accurate description, we can borrow the description of the Scottish philosopher "Thomas Carlyle" (1795 - 1881), who said: «He is unique, a messenger from the world of supernatural to the humanity; the words spoken by him are not for anyone else; his words emanate from the core of the facts; he sees the interior of everything; his sayings are a kind of the inspiration; he came from the heart of the world and from the intestines of the universe; he is a part of the 
essential facts of things».. ${ }^{49}$ Thus, if the news, orders and prohibitions came down from the upstairs people to the downstairs people, then, the lucky is the one who meets the news with the validation and acceptation, and the orders with the submission, and the prohibitions with the glorification. ${ }^{50}$

All that in fact is not from Islam at all; the Prophet moved to the Supreme Comrade without mentioning the successor after him. He did not recommend who will take care of the Muslims after his death, as there is inheritance of the governance in Islam. He has stressed the importance of consultation (al-Shura), then said: «There are no people consulted with each other, but Allah guided them to the most right guidance of their affair», as a realization of the Quranic verse:

They adore Him with appropriate acts and rites and conduct their affairs and ordinary pursuits of life by taking counsel together to establish justice and integrity

(Al-Shura $=$ Counsel, verse 38).

He also warned against trying to assume the Principality, saying: "It is a trust, and on judgment Day it is a shame and regret, except who has taken it with its right and performed what he must do in»".

When 'Abu Bakr' (573 - 634) assumed the caliphate, he delivered an address, in which he stated: «O people, I have taken care of your affairs, and I am not the best of you; if I do well, you should support me, and if I turn away from the truth, you should adjust me», hence he said: «Obey me as long as I obey Allah and His messenger; if I disobey Allah and the messenger, you should not obey me».

What we would like to say by our separation between the concept of Islam and the concept of Muslims, and by the examples above, that if there are reasons for the deterioration of the Arabic - Islamic civilization, and for the shameful image of backwardness of the Muslims today, these reasons do not refer to Islam as a religion, but to the wrong 
applications of its teachings, or rather to the absence and falsification of application, otherwise, it was impossible for the Prophet and His companions, and those closest to them spiritually, mentally and temporally, to build the giant edifice of civilization we are talking about. Perhaps this meaning becomes clear from a story reported by Dr. 'Zaki Naguib Mahmoud' (1905 - 1993) in his book Renewing the Arabic thought. He stated: "Islam is mentioned, in general without any of its details, just one day when I was in a group of university professors in the United States of America, and I felt something of the silent rejection in the faces. Soon, the circumstance became adequate in order to mention something of the details that define the general features of this belief, and I have done, then, one of them said: if that is Islam, then are we Muslims and we do not know?. At that time, I remembered what one of our masters, perhaps Imam 'Muhammad Abduh' $(1849-1905)$, that you may find in some European countries Muslims without Islam, as you may find in some Arab countries Islam without Muslims. This means that Islam is a set of values that I think that there is no one on the surface of earth rejects anything from them, in view of the fact that they are ideals ... and you may find among who reject Islam as a pure idea who are living those ideals actually, and find among who embrace this Islam itself as a pure idea who are not living anything from its values». ${ }^{51}$

Then, we should not ascribe the error to Islam whereas its people are far from it, and we can not blame Islam whereas its people are not sincere believers. Islam can not be criticized whereas its judgments are inoperative, but the error is carried on the Muslim who knows that he is a Muslim, but he does not know Islam nor its requirements, namely that between him and Islam the distance of his knowledge of the existence of Islam in his soul!. 


\section{The Start of Combination among Nations}

The Arab invasions and conquests led to a strong process of mixing between Arabs and the other peoples who are more advance and civilization than them; where Sham, for example, was a Roman territory which educates with the culture of Romans, and embraces the Christianity after many civilizations had rotated upon it. Egypt, also was an ancient cradle of civilizations by its privileged geographical location, especially that Alexandria is located in it. As we know, Alexandria was the centre of philosophical systems and the different religious denominations.

The process of mixing was not limited to the mental opinions merely, but also extended to the religious beliefs, social and economic systems, as well as the genetic and biological mixing. It has helped to this mixing through many elements, the most important of them: ${ }^{52}$

- The tolerant teachings of Islam in the conquests.

- The entering of many people from the conquered countries into Islam.

- The mingling between Arabs and others in the dwelling and the conditions of life.

As a direct result of these factors, Islam become a melting pot of different culture, philosophies, systems, laws, hopes and languages. Even if you look at the intellectual life at that time, you see it as the sea: «its surface is very homogeneous; because the water is its chief element wherever you direct your vision in its sides. But if you sink under the surface in order to look for the routes of life inherent in it, you will find them on a great degree of the conflict and the severity of discrepancy, even you wonder: how can these fighting and opposite livings are components of one sea».

In other words, Islam became under the conquests as one huge tree, if you see it, you know its identity, and realize at first glance its constancy and the firmness of its roots, but in fact, it undergoes continuous internal and external interactions and changes. If the nature of tree, as a living being, that it 
feeds on what is appropriate for its body, and requires a climate that serves to its life and growth, it may happens sometimes that it coincides with the food that it fails to assimilate, or coincides with a climate delays its growth, then it seems with shriveling and falling leaves.

I presume that the tree of Islam was assimilated the whole cultures, philosophies and sciences that it received, then the circle of its production widened and diversified. Yet, it eventually retreated as a result of a spoiled climate that it had no ability to sustain, even though it remained able to overcome it. In fact, the image of tree here is not "Parmenidical", because it actually changes and moves, as well as it is certainly not "Heraclitical", because, unlike the river of Heraclitus, it remains the same throughout the continuous change that it pass through, ${ }^{54}$ therefore we say that it is a neutrosophic tree.

\section{The Movement of Translation}

There is no local culture, which had reached the stage of internationalism, but its internationalism was a result of its interaction with other cultures by the mutual influence. The way to do so is translation, because it is the primary means through which the intellectual communication among different nations occurs, and it is the factor which forms the basis of cross - pollination and the mental production among different and successive generations of the human beings. That is what Muslims and Arabs have done during the golden ages of Islam, and that is what Europe has done in the Renaissance era, which began with the scholastic era, and reached its peak during the fifteenth and sixteenth centuries A. D.

By means of the translation, the Arab - Islamic civilization has been able to contain "the other" mentally, after its conquests had allowed it to contain him spatially and organizationally. The comprehension in the first case means the ability to assimilate the ideas of others, the difference with them and the addition to them. In the second case it means the 
genius of dealing with others, not from the perspective of the arrogance of power, but from a religious and political perspective, which guarantees the freedom of religious belief and opinion, and makes room for the pluralism - in all its meanings - in one large society, ${ }^{55}$ as a confirmation of the Quranic verses:

You have your religion and I have mine $($ Al-Kafiroon $=$ The Infidels, verse 6$)$

People were but one single nation, then they differed among themselves in opinion and each group cherished a certain view and belief and were altogether set at variance. Had it not been for Allah's word proclaimed beforehand to delay the decision on matters of doubt and dispute among them and to put punishment in respite, their Hereafter would have been planted in the now

$$
\text { (Yunus = Jonas, verse 19) }
$$

And had Allah, your Creator, willed, He would have made mankind one single nation conforming to one religion. Nonetheless, their innate incongruity would have induced to be thus still at variance

(Hud, verse 118)

Although the movement of translation into Arabic has historically been associated with the Abbasid age, in particular the era of the Caliph "al-Ma'mun" (786 - 833), there were strong initiatives before that all over the Middle East. In the era of the Umayyad, who ruled from Damascus, the Islamic empire already encompassed large areas - including Egypt, Syria, and Persia - that had come under the influence of Hellenism from the time of Alexander; and before the ninth century was over Islamic rule had reached Kashmir in the east and Khwarazm to the north. Then the translation began with order from "Khalid Ibn Yazid Ibn Muawiyya" (635 - 704), who asked some Greeks living in Egypt to translate some Greek books in chemistry into Arabic. However, the 
movement of translation of the Umayyad was not but individual attempts, which die with the death of who performs them. They also were restricted only to the practical sciences, such as the handicraft (Alchemy), the Medicine and the Astrology. ${ }^{56}$

In the early Abbasid period the higher administration of the court itself was in the hands of cultivated Persians who had gained much favor and influence with the Abbasid rulers and whose intellectual interests inclined them to various forms of secular learning and to a rationalizing approach for understanding matters of religious belief. Some of these Persian officials acted as translators, especially from Persian, and in general they constituted an important, politically influential part of Baghdad's intellectual elites. Two other groups within the empire (and concentrated in Syria, Iraq, and Persia) had maintained a long - established tradition of Hellenized Syriac learning. One consisted of Christian physicians and Christian theologians, who continued to pursue their interests in Greek logic and philosophy in scattered monastic schools; the other was the pagan Sabians of Harran, in northern Mesopotamia - an ancient Semitic group - whose astral religion connected them to Hellenistic astrology and astronomy and to Hermeticism. It was from these two last groups that the Abbasids were able to recruit the scholars who carried out the translations of Greek Medical, Philosophical, and Mathematical works into Arabic, either from pre-existing Syriac versions or directly from the Greek. ${ }^{57}$

One might then say that the stage was set, at a certain place and time, for the translation movement that began during the Abbasid era at an unprecedented pace. The main motives for that were; the Muslims had deepened in civilization which based on science, then they looked for it at its people from the former owners of ancient civilizations. The religious movement reached in the late of era of the Umayyad an advanced standing, where the Muslims spoke in philosophical issues (such as "Fate" and "free will"). The controversy 
aroused between them and the others from other religions who had lived under the parasol of Islam (such as "Judaism", "Christianity", "Zoroastrianism", "Madianism", "Manicheism", ... etc.). It was inevitable that Muslims be armed with the logic and philosophy, not only in order to defend the Islamic faith, but also to offer and define it in phrases borrowed from the tongues of its opponents.

The result was a huge intellectual ferment, centered especially in multicultural Iraq, to which the movements of Islamic theology, philosophy, and science owed their birth.

Moreover, some Caliphs of Muslims, especially the Caliph al-Ma'mun tended to philosophical sciences, so they encouraged the translators to translate them into Arabic. The generosity of Caliphs in the treatment of these translators was great. They established for them libraries, such as the house of wisdom (Bayt al-Hikma), and awarded them the huge money, to the extent that "Hunain Ibn Ishaq" (Johannitius) (808 873) was receiving a gold equal to the weight of his translations. That ought to tempt the translators rushing in translation, but this did not happen. They were usually committed to accuracy and sought to the uprightness in what they translated, as well as they were keen to have the original copies of which they translated, and they divided the sentences into sections, chapters, and paragraphs, so that the translation of their meanings into Arabic can be easy and clear without any confusion. Their explanation of the original certifies that they were familiar with the expressions of vernacular and the familiar terms in the language that they translated. As for the mistakes that emerged in some translations, they were caused by three reasons: ${ }^{5}$

- Many of the books of Greek heritage were translated into Syriac before, and their translators had fallen in mistakes. When the Arabs translated these books from Syriac, the mistake moved with them to the language of Arabs.

- The Arab translator were often more satisfied with the translation of the important meanings and neglect else 
deliberately, not out of ignorance or misunderstanding. Yet, their unrestricting with the text made the translations in some cases clearer than the original text.

- Most translators were careful to explain the text in the course of translation, and to examine and criticize it, and to add to the original the meanings that they got by the experience, without the guidance of the reader to pay attention to what they added to the original from their own meanings and ideas.

Apart from this, many Arab translators, like "Hunain Ibn Ishaq" and his school, "Thabit Ibn Qurra" (826 - 901), and "Qusta Ibn Luqa" (820 - 912), were famous for the honesty, accuracy and the ability to understand the original, and to express it in an obvious literary Arabic.

Thus, the heritage of Arab - Islamic received the heritage of ancient civilized nations, especially Persia, Greece and India, and the culture of Islam contacted with all these tributaries and created with them in the light of the sensory experiences and mental reflections of Arabs, from which it was that philosophical - scientific heritage abounding with the originality and innovation.

\section{The Internal Structure}

No nation has widen with its conquests, has extended with its extremities, has maintained them, and has absorbed the incoming thought ..., but was accompanied by an internal powerful structure, with the ability to move and shape what allows it to withstand the winds of change and evolution. It has the flexibility to make it able to adapt with its inner interactions, and to receive the external zygotes, filtering and retaining the good of them in the light of its actual needs.

We do not mean here with the structure 'the political regime over the age of the Islamic empire'. It was mostly, as we stated, a distorted application of the principles of Islam and its real judgments, but rather we mean the general characteristics 
that shaped the Islamic mentality and distinguished it with a genuine thought that combines science and philosophy.

The first of these characteristics is that Islam did not take the truth in the abstract theoretical thinking only, as was the case with Greeks, but pushed the Muslims to the concrete realistic speculation. Thus, if 'Aristotle' has gone to that there is no awareness but with the universal, Islam has declared clearly:

Indeed, he faculties of hearing, sight and intellect, each and all, are held responsible in Day of judgment and shall have much to answer for

$($ Al-Israa $=$ The nightly journey, verse 36$)$

And if Islam describes who are not prudent, it describes them as:

Deaf and dumb, insensible to light, they are unable to retrace their steps that lead them into error

(Al-Baqarah The heifer, verse 18)

Namely, that it underlines the importance of observation as a methodical requirement that is closely related to the mind. Therefore the verses which mentioned the sun, moon, stars, and winds, were as a guide for Muslims to research in the Astronomy, and the verses which mentioned the cattle and sea animals were as a motive to research in Biology, ... etc. Thus, Islam awakened an experimental spirit in an ear that was refuses the world of sense as it unprofitable for the human thought, hence, emerged the concept of observation and experiment initiated by the modern European Renaissance.

On the other hand, and as a second property, previous nations asked their Prophets to perform miracles as a condition of belief; Mussa's staff that counter - worked their ingenious expedience and swallowed all that they cast upon the ground of fraudulent devices of a mean and deceptive kind; The miracle of Christ in raising the dead; Even the disciples of Christ after they have believed, asked him to send 
down from heaven a table. But In Islam, the divine call was clear and frank: the mind and logic must be taken the place of the invisible or the supernatural:

Invite people $O$ Muhammad graciously to the path of Allah, the path of righteousness, with wise, graceful and appropriate inducements meeting them on their own ground and propitiating them, each in his own capacity, to open their hearts' ears and their minds' eyes. Reason with them in a logical, peaceable and gracious manner

$$
(\text { Al-Nahl }=\text { The Bees, verse 125) }
$$

The humanity has reached with Islam the stage of maturity, so it no longer need to be guided by the emergence of a miracle or a supernatural principle, hence, the birth of Islam was means at the same time the birth of inferential controversial mind with all its dimensions.

The third pivotal property of the Islamic mentality formed by Quran, is the Islamic portrayal of the flowing cosmic movement:

Another outward and visible sign of an award and spiritual grace, is the night We strip of daylight and there, they are driven into darkness. And the sun runs its course and describes its orbit, sited in a settled position, belonging, as determined by Al-Aziz, Al-Alim. And for the moon We have determined mansions or divisions of the ecliptic which it occupies on 28 successive days and there it falls in a swoon and be like shriveled and withered date stalk

$$
\text { (Ya Seen, verses 36, 38, 39) }
$$

Even the human history, Quran alerts us that it is mobile in turn:

We rotate fortune and alternate the days of gratification and the days of distress

$$
\left(A l-{ }^{`} \text { Imran }=\right.\text { The family of Imran, verse 140) }
$$


Yet, the movement here is not simply a circular movement (as the idea of Great Year with Heraclitus), but it is a developed renewed movement:

He adds, joins or unites one thing to another at creation so as to increase the number, the size or the importance of a given created being or beings as He will Allah is indeed Qadirun (Omnipotent) over all things

$$
(\text { Fater }=\text { The Creator, verse } 1)
$$

This means that the truth has not been occurred at all, but it is always on the verge of the occurrence. This movable description has been manifested, including the human mind, in the emergence of the endeavor (Ijtihad) as a third source of the legislation; Islam was declared the end of the Prophecy, and that the scholars are the inheritors of the Prophets, therefore, the guidance did not become dependent on waiting the advent of the Prophet; it is constantly renewed and available by virtue of the scholars. In Ijtihad, the religious text is not, and was not, taken as a general rule or premise, so that we say, as in the Aristotle's syllogism, that what applies to general applies to the particular, but the general rule is that the legislation was found for a wisdom or interest. If the obligation with the text leads the loss of wisdom or interest, then the application of the text stops. For example: if the divine text is binding the people to fulfill their vows as stated in Quran:

\section{And to fulfill their promises and pay their vows}

$$
\text { (Al-Hajj = Pilgrimage, verse 29), }
$$

This text has been drafted in a general rule: «everyone who made a vow must pay it», then in a formula of an Aristotelian syllogism: «His man made a vow, then he must pay it»; but it must be observance the special circumstances; it is not permissible, for example, to pay a vow in the guilt of Allah. As well the Messenger did not permit the pricing of goods, because the seller under this condition becomes forced to do 
what is inconsistent with his right in the ownership, but the jurists after Him gave a formal legal opinion that the pricing is permissible, because it is not permitted for the seller to take any gain that will be to the detriment of people. And while the Prophet had legalized the testimony one to his relative in the judgments of inheritances, where people were preferred to speak the truth in testimony on the kinship, the jurists after Him gave a formal legal opinion that is not permissible, where some people choose to support their relatives to tell the truth ... etc. ${ }^{59}$

With this mentality, the thinkers of Islam disagreed and excelled. They knew that their superiority existed in their disagreement, and that in their mental and jurisprudential difference was mercy to Muslims, as well as a confirmation of the multiple and flowing facts of truth. The aspects, causes, and consequences of difference were various. There is, firstly, the linguistic side; its examples: their difference in the verbal concept of the precise and exactly defined verses, and the allegorical ones in Quran, which led them to speak about the humanization (or anthropomorphism) and absoluteness (or the superlative qualities). There is also their difference in the linguistic interpretations of the destiny (the divine decree and the predestination = al-Qada wa al-Qadar), which led to the problem of determinism and freedom of choice (al-Jabr wa al-Ikhtiyar). As well as their difference about the concepts: "Believer" (al-Mu'min), "Infided" (al-Kafir), and "Grave sinner" (al-Fasiq). These concepts resulted the problem of the intermediate position (al-Manzilah bayna al-Manzilatayn); and their difference in the meaning of "Dissent" (al-Khurouj), "Postponement" (al-Irjaa), and "Withdrawal" (al-Itizal), which resulted the origin of enjoining the good and forbidding the evil (al-Amr bil ma'ruf wa al-Nahy an al-Munker), ... etc.

There is no doubt that one of the most important reasons for this difference is the multiple races within the Islamic state, between pure Arabs, who are different in their linguistic 
structures, and hybrid ones, who dealt with this linguistic structure differed about it completely.

Secondly, there is the political side, where the political dissents erupted after the death the third Caliph "Uthman Ibn Affan" (574 - 656). The result was the formation of Islamic doctrines and sects: Indeed, Shi'ism with its different philosophies and sects, such as "Twelvers" (al-Ithnãashariyya), "Ismuilism", 61 and "Fivers" (al-Zaidiyya), ${ }^{62}$ has arisen and developed as a result of political origin. However, the Greek doctrines and Gnosticism ${ }^{63}$ have generated in the philosophy of Shiite to support the idea of protected leader (al-Imam al-Ma'sum), as well as for the sect of al-Khawarij (those who repellers) ${ }^{64}$ and the sect of al-Mu'tazilah ${ }^{65}$, who were motivated by political orientations. Most of what happened in the history of Muslims of vital intellectual revolutions, but turned to political revolutions: al-Kaysani Shi' ${ }^{66}$ and their successor the state of al-Qaramita ${ }^{67}$; Ismailis and their successor the state of Fatimids ... etc.

Thirdly, there is the economic side, which has also begun to emerge in the era of "Uthman Ibn Affan", where the feeling of deprivation by the masses of poor people led to the establishment of Shi'ism and rallying the poor public around "Ali Ibn Abi Talib" (the fourth Caliph) (599 - 661).

This was demonstrated when "Ali" equaled between the rich of Sahaba and the poor of Muslims, which urged "alZubayr Ibn al-Awam" (593 - 656) and "Talha Ibn Ubayed Allah" (596 - 656) to the uprising against "Ali", and fuelling the flames of the dire war against him. It is also said that the basis for al-Kaysani Shi'a, which is a major movement in the history of Islam from which stemmed the revolution of alQaramita and the revolution of Negroes, but because the equality between Arabs and non-Arabs in funds by "alMukhtar Ibn Abi Ubayd" (622 - 687). In some historical models of the early life of Mu'tazilah, that the basis of its rise was an economical factor that was the carelessness of State's money by Sultan, and not to evenly distributed among 
Muslims. And it justified the rise of Fatalism in the same economical justification, and perhaps the word of "Ma"bid Ibn Khalid al-Jahni": "These Kings shedding the blood of Muslims and take their money, then they say it is a work of God" is the basis of the doctrine of free will in the Islamic world; and from this the word 'Mu'tazilah' was created later. ${ }^{68}$

In fact, we can not deny that a lot of Muslims were occupied with the materialistic world after it came to them in its all goods. Then, they quickly differed with each other about it; each of them wanted to legislate for himself and for his method in the life. That was a reason for the entry of external ideas, approaches, and philosophies in the Muslim community, and the owners of these external mental yields tried, and succeeded in part, to remove some Muslims from the circle of actual Islam. Yet, that does not mean that this difference, for the great majority, was a religious doctrinal one, but was, in fact, a verbal difference for some time, and a natural difference for another time, or rather we can say that it was a difference in the religious thinking, not in the religion itself. It was a difference in the means by which the religion is served, whichever is the earliest and most effective approach to achieve the purpose of religion and its method.

For example, any Muslim never disagreed with another Muslim that the judgment is a part of the religion, namely that Islam is a law (Sharia) and doctrine (Aqida). They never disagreed that the Quran, which is preserved and existed by Allah in heaven's realm, is what we should seek a decision from, but Shiites saw that "Ali" must be the first Caliph, while Kharijite saw that any Muslim from any race can be the caliph, and it is true that we work with him. Did the difference relate to the essence of religious faith and the teachings of Sharia? Or it is a political dispute that could have been settled by a thousand means but the sword?

No Muslim and Muslim disagreed that Allah is fair, and $\mathrm{He}$ judged with the reward of submissive and the punishment of disobedient, and told us that in His holy book, then that 
became a legitimate duty for us. But in no time the silly disagreement erupted between Mu'tazilite and Sunni: Is that a God's duty?

Then we can say that there are no religious sects in the meaning that the Islamic nation ramifies as the River Nile ramifies in its lower stream to branches and canals, but there are schools of thought, jurisprudential systems, or limited practical differences. When this disagreement is deprived from the political factors that froze it to remains, or duplicated it badly, then things would seem normal. ${ }^{69}$

\subsection{The Philosophical Cultures in Islam}

Muslims had a package of philosophical cultures that have had an important impact on the enrichment of Islamic intellectual life over its thriving centuries on the one hand, and in the expression of the originality of Islamic thought and its character on the other. It is certainly difficult to provide a detailed presentation of these cultures, with the multidimensional, in a few leaflets remainder of this chapter, so we shall only refer to their main types and features, as a prelude to the presentation of detailed models that show their nuetrosophic feature during chapter three.

Dr. Ali Sami al-Nashar restricts these cultures, in his book The emergence of philosophical thought in Islam, in six types, they are: ${ }^{70}$ Islamic philosophy, Islamic mysticism, religious science (Elm al-Kalam), the science of fundamentals of jurisprudence (Elm Usul al-Fiqh), sociology or philosophy of politics or philosophy of history, and the philosophy of grammar. We'll have a look at each one briefly:

\section{A. The Philosophy of Islamic Philosophers}

The philosophy of Greek moved to the Islamic world through a comprehensive and nature process of naturalization, which is described by one of the historians as «the most astonishing event in the history of thought». ${ }^{71}$ 
Perhaps the motive of astonishment, I think, is the ability of Arabic - Islamic mind to receive the incoming culture and to know it from all directions, and then direct it according to the Arabic local environment, which is different with that produced the Greek thought before; namely in the light of its own concerns, problems, and questions, as well as its free choices in a cultural surroundings that characterized by the plenty and diversity.

With the philosophers of Islam, we mean here those who have borne the burden of translation, explanation, and examination of the major Greek systems to the Arab - Muslim reader, and those who were fully aware that they were facing a strange pattern of thought, came to them, not as an invading force setting off from a powerful stronghold in Alexandria, Antioch or Harrãn, but rather as an invited guest. Despite the early caution from this guest because of the gap that appeared immemorial between him and the doctrines of Islam, but it quickly proved to hold an attraction for its hosts far beyond the promise of his practical abilities, which is manifested in the writings of a large number of powerful Muslim thinkers whose allegiance to a comprehensive Hellenistic view of the world of matter and thought and values can be described only as a thoroughgoing commitment, such as: "alKinidi"(Alkindus) (801 - 873), "al-Farabi” (Alpharabius) (870 - 950), "Ibn-Sina" (Avicenna) (980 - 1037), "Ibn-Bajja" (Avenpace) (1095 - 1138), "Ibn-Tufail" (Abubacer) (1105 1185), "Ibn-Rushed" (Averros) (1126 - 1198), and others.

These thinkers engaged in a systematical method and they tried in its light to conciliate among the various Greek systems, then tried to reconcile between them and the doctrines of Islam in every possible means. Their project was a mixture of the Aristotelianism, Platonism, and NeoPlatonism, but in fact it appeared for others as an incomplete and distorted project. It must be sharp clash among philosophers themselves from a point of view, and between them and the refuters of playing with the Islamic law from a 
pure mental view; those who saw in the incoming thought a threat to the safety of doctrine, on the other. This clash reached the peak when Imam "Abu Haid al-Ghazali" (1058 1111) published his famous book "Incoherence of the philosophers".

In this book, al-Ghazali begins his dialogue with the reference to that the plunge into the story of difference of philosophers requires a long time and a lot of effort: «their errand is long, their dispute is many, their opinions are spread, and their routes are faraway and back to front ...». ${ }^{73}$ Then he starts to identify the points of disagreement among them, i.e. the philosophers, and the others of the other sects, where he classified them in three sections:

1. A section in which the disagreement is due to a pure term; such as their designation of the Creator of world - He is infinitely far above the impiety they utter against Him - as a substance, with their interpretation of the substance as existing not in any object, i.e. who can exist by himself without the aid of any other thing. Al-Glazali refrains from delving into this section because it requires another field, which is the field of linguistic and jurisprudential studies. $^{74}$

2. A section in which their doctrine does not clash with any principle of the religious ones, and the necessity of validation of Prophets does not require dispute them in it; such as their saying that the lunar eclipse is the disappearance of moonlight as a result of the passing of moon between the earth and the sun, as it quotes its light from the sun ..., al-Ghazali refrains from delving into this section also, because it does not affect the core of belief. ${ }^{75}$

3. A section in which the conflict is related to one of the principles of religion; such as their speech on the contingency of world, the attributes of the Creator, and the thronging (or the resurrection) of bodies. They denied all that, therefore al-Ghazali makes this section is the axis of his book. ${ }^{76}$ 
Al-Ghazali confines the total of philosophers' fallacies, in the section of theology, in twenty issues, he judges that they are infidels in three of them, and they are innovators in the rest. The three blasphemous are:

1. Their saying that the material world is eternal and old and the whole of substances are old.

2. Their saying that God does not take note of the incident particulars, and He knows only the universals.

3. Their denial of the resurrection and the thronging of bodies.

Overall, what we can conclude from the attitude of alGhazali that although he was one of the strongest opponents of Greek philosophy in the history of Islamic thought, his opposition did not go beyond the limits of the divine side. As for the product of philosophers apart from that, such as mathematics, logic, physics ...etc., he did not go into the attack on them or even the refutation of them, but he urged to them in many books. The introduction, by which he started his influential work on Islamic jurisprudence "al-Mustasfa" (The pick), was a crucial point for the history of logic in Islam, where he went so for as to say that without logic one could not be sure of any part of knowledge (whether secular or religious, theoretical or practical). Al-Ghazali was able to do this because he understood logic as a mere instrument, a kind of "balance" for weighing arguments which did not commit its user to any doctrine or belief. ${ }^{78}$ This balanced attitude urged him to warn of two interrelated and opposite dangers: the first is the danger that some people see the accuracy of the mathematical and logical proofs of the philosophers, then form a good opinion on all their sciences, even though their accuracy in a side does not require their accuracy in other side. The second is the danger that some people turn to support the religious belief by ignorance, then think that the support of belief requires the comprehensive denying of what the philosophers said, including what depends on a critical proof such as logic and mathematics. ${ }^{79}$ 
In other words, the aim of al-Ghazali was to demonstrate the mind as unable to capture the divine truths, therefore he tried to snatch the people's confidence in the mind as a source of divine matters; but, in doing so, takes the mind itself as a mount to reach this goal. That means clearly that he philosophized to refute some of the conclusions of philosophers, which reminds us of the words of Aristotle: «Let us philosophize if the matter requires the philosophism; unlike that, we have to philosophize in order to prove that the philosophism is unnecessary». ${ }^{80}$ Then, the naturalization struck those who refused the process of mixing between the Greek metaphysics and the metaphysics of Islam, with alGhazali leading them, as it dominated before those who brought the Greek thought and showed enthusiasm for it from the philosophers of Islam. One of the vital features, that the Arabicized name, falsafa (= philosophy), and its all derivatives, such us the plural noun (falsafat), the adjective (falsafi), the nomen agentis (singular and plural: falylasũf and falãsifa), and even a verb (tafalsafa: to philosophize), all of which were and still are used in Arabic literature as commonly understood words. The continuing existence of these words in fact indicates a deeper process of assimilation through which elements of falsafa have penetrated, in various degrees, practically every department of Islamic thought. ${ }^{81}$

In addition to that, the philosophy, as understood in the Arab - Islamic world, was not only a research in divine metaphysics, which has stirred some, but it has also included all types of scientific knowledge that Muslims were excellent in them. Despite the distinction frequently expressed in medieval times between "mathematicians" (al-ta'limiyyun) and "natural philosophers" (al-tabi'iyyun), it is known that the interests and activities of these two groups often overlapped to a significant extent. Indeed, the "mathematical sciences" were considered a sub-class of the "philosophical sciences", which also concluded natural philosophy (tabi'iyyat) and metaphysics, and the whole constituted what was referred to 
as "the sciences of the ancients" (ulum al-awa'il), or "the ancient sciences" (al-ulum al-qadima). It is significant in this regard that the scientists/mathematicians and the philosophers were not only interested in and often deeply influenced by each other's fields. Thus, for example, it may be observed on the side of the falasifa, that al-Kindi wrote much more on the empirical and mathematical sciences than he did on falsafa; one of the most distinguished contributions of al-Farabi was a substantial book on music - a mathematical subject (the book of music = kitab al-Musiqa); Avicenna carefully studied Euclid (325 - 265 B.C.) and Almagest, as attested by his alShifa (The book of healing), and seriously involved himself in problems of astronomical theory and observations; and Averroes' interest in problems of the mathematical study of the heavens led him to promote an alternative to the Ptolemaic system which inspired a contemporary mathematician, alBitruji (Alpetrogius) (d. 1204), to construct such an alternative. On the other hand, "mathematicians" like Thabit Ibn Qurra, Ibn al-Haytham (965 - 1039), al-Biruni (973 1048), al-Khayyami (d. 1131), al-Tusi (1201 - 1274), and alShirazi $(1236$ - 1311) are known to have written on many subjects that fall within the province of falsafa, including "Natural philosophy" and Metaphysics, and on Religion. ${ }^{82}$

\section{B. Islamic Mysticism}

Islamic mysticism is divided into two distinct sections; the first is the Sunni section, which is based in its origin and evolution on Quran and Sunnah. This section formed the "ethics" in Islam; the second is the philosophical section springing from the first one in some of its stages of development. It includes a mixed set of Greek thought, especially Neo-Platonism and Hermeticism, and Eastern Gnostics thought: Indian and Persian, then zygotes of Judaism, Christianity and Islam. ${ }^{83}$

The two sections share in saying that there is a direct interior experiment to the contact between the slave and his 
God. It requires the acquisition of a private faculty unlike logical reason to achieve this contact, which is known as the faculty, or the instrument, of intuition.

Concerning this approach, mystics distinguish between two types of the science of law (Shariah): science of appearance and science of interior; the first relates to the apparent deeds, i.e., the deeds of organs which are the worships, transactions and the legal judgment. It is a speciality of jurists. The second includes everything related to the interior deeds, namely the deeds of hearts such as ranks, conditions, controls, and suppression of desires. It is a speciality of mystics themselves. ${ }^{84}$ This means that the knowledge to which the mystic arrives is a direct knowledge, not based on premises or propositions or proofs. It is a supermental knowledge, not owned by anyone but who follows the path of mysticism; that is inspired the direct knowledge, therefore it is called "the revelation". It is one of the endowments of God and His generosity, and comes only after the purity of heart, where the lights illuminate it by Allah: the One and the Right. If one reaches this rank, he is called "knowledgeable" (al-Arif). ${ }^{85}$

However, the two former sections of mysticism differ thereafter in what are adopted by the philosophical mysticism of alien beliefs to Islam and contrary to its explicit beliefs, which are contained by Quran and confirmed by Sunnah; the most important, of course, the doctrine of incarnation and the doctrine of pantheism.

Concerning the doctrine of incarnation, Islam does not agree to the incarnation of Creator in the creature or the absorption of creature in the Creator, because each of them have its nature, therefore, Islam denied the Christian idea of incarnation and what it requires of transfer, motion, and partiality, which are impossible for Muslims to Allah. As well as Islam is not consistent with the tenet of pantheism, because it requires the transition from its original faith: «there is no deity but Allah», to the philosophical doctrine of mysticism: «there is no existing in reality but Allah»; the context of each 
of them leads us to results that are strongly contrary to the other. ${ }^{86}$

Sufis adopted many ideas that are not accepted by the other Muslims. These ideas include the distinction between appearance and reality, the psychological behavior abounding with reconsideration, education of the heart to rid itself of baser instincts, the love of God, approaching God through progressive stages (Maqam) and states (Hal). Because of these ideas, in addition to what infiltrated the philosophical mysticism of ideas and doctrines that stab in the purity of belief, it was natural that the mysticism is attacked by who did not behave this way and did not follow this trend in the understanding of religion and ideal life, in particular the jurists.

Perhaps the most prominent and broader attack in this regard is that done by "Abu al-Faraj Ibn al-Jawzi" (1126 1200) in his famous book titled «Delusion of the Devil» (Talbis Iblis), as well as Shaikh al-Islam "Ibn-Taymiya" (1263 - 1328) in more than one subject of his legal opinions (Fatawa). Overall, the first went to that they have switched from knowledge to deed, and devoted themselves to preachments instead of the knowledge of Quran and Hadith; they passed the border in matters of worship and called for nonobservance the reasons and omission the precaution in funds; they took particular forms in the clothing and eating, and preferred the isolation, and got engaged in jugglery and dervishing, ... etc. ${ }^{87}$

Ibn Taymiyya has denied what is previously denied by Ibn al-Jawzi, and launched a harsh attack on each of "Ibn Arabi" (1165 - 1241), "Ibn al-Farid" (1182 - 1235), and "Afif al-Din al-Tilmisani" (d. - 1291), for their saying with the incarnation and pantheism, but he distinguished, in spite of that, between the correct mysticism which is acceptable, and the deviant mysticism which is reprehensible: «the right that they strive in obedience to Allah, among them we find the guilty and the pious ...»; and he approved the miracles of the chosen people 
of Allah: «the miracles are right according to the agreement of the people of Islam and Sunnah and community ..., but they do not indicate the inviolability of their owner, nor to be followed in all what he say ...», i.e., Ibn Taymiya did not attack the mysticism as a mysticism, but he attacked the mystics' deviations that are not approved by law (Shara). ${ }^{88}$

Despite the aspects of mysticism that contrary to the teachings of Islam, and the extravagance of some mystics in the influence with the philosophical theories, and the immoderation of some jurists in criticizing and questioning their beliefs, ..., in spite of all that, Islamic mysticism performed its role in spreading Islamic call outside the homes of Islam, and in establishing the idea of "conscience", which had not been known to Europe but recently at the hands of the English moralist "Buttler" (1692 - 1752), and in calling for the universal human tendency that open-minded to other societies and races, to achieves what is so-called by "Bergson" "Sociète ouverte" in his "The two sources of morality and religion".

\section{Ilm al-Kalam}

Ilm al-Kalam (scholastic theology) or Ilm Usul al-Din (= the way of life in submission of Allah) is the science of defence of the faith beliefs by means of the mental evidences. Or, in other words, it is an inquiry into God, and into the world as God's creation, and into man as the special creature placed by God in the world under obligation to his creator. It follows from this description, or, if you like, definition, that Kalam is a theologically inspired and theologically oriented form of thinking - not only when it speculates about God's unity or the relationship between his essence (dhãt) and his attributes (sifãt), but also when it gets engaged in subtleties concerning the ultimate constituents of the world, or when it asserts or denies human freedom or moralizes about human behaviour, or legislates for the governing of Islamic society. ${ }^{89}$ 
Kalam has arose in an atmosphere of religious conflict, political and non-political, among Muslims themselves on one hand, and between Muslims and non-Muslims on the other hand, where the fathers of the Church tended, since the entry of Muslims in their countries, to attack Islam by means of philosophical arguments, and to the controversy about the "nature of Christ", the "Word", the meaning of this nature and the meaning of "Word" in the writings of Muslims themselves.

This has led Muslim theologians (al-Mutakallamun) to arm themselves with the weapon of their opponents; therefore, Kalam becomes famous as a speech to defend the religion, as well as the emotional controversy. This view was encouraged by the enemies of Kalam, especially the falasifa, or by individuals who had no great sympathy or much use for it, e.g. Ibn Khaldun. But this view in fact, has tended to trivialize kalam as an intellectual pursuit, and as a domain in which the Muslims excelled more than any other intellectual domain, but this view certainly precludes a full understanding of the nature and consequences of kalam's interactions with other competing pursuits, especially falsafa.

Whatever the reasons that lay behind it, the prevalent view flies in the face of the historical and the textual evidence available to us. Take, for example, the earliest school of kalam, the Mu'tazila ${ }^{90}$ here we find ourselves in front of five bases or principles (usul), non of which is specifically apologetic or polemical in character. They are: ${ }^{91}$

- Monotheism (= al-Ta whid).

- Divine justice (= al-Adl).

- Promise and threat (= al-Wa'd wa al-Wa'id).

- The intermediate position (= al-Manzilah bayn alManzilatayn).

- Enjoining the good and forbidding the evil (= al-Amr bil ma'ruf wa al-Nahy an al-Munker). 
With monotheism, Mu'tazilis means the absolute unity and oneness of God; i.e., the exclusion of any from of plurality (Shirk) or anthropomorphism (tashbih) from the godhead. This principle led the Mu'tazilis to urge the explosive doctrine that the Quran, as the speech of God, is not eternal but created, the doctrine that was the reason for the ordeal of Iman "Ahmed Ibn Hanbal" (780 - 855) and all Hanabilites, for the denial of the belief that the creation of the Quran means the verification of its oldness; and since everything that is old is deity, the God's possession of the deity requires that $\mathrm{He}$ is the only old, and the saying that the Quran is creature, which is consistent with their concept about the monotheism. ${ }^{92}$

The second principle, the divine justice, goes to that Allah is Benevolent and the Benignant Sovereign to His servants; God does no evil, and He never demands from any human to perform any evil act. Then, if man's evil acts had been from the will of God, the punishment would have been meaningless, as man performed God's will no matter what he did. That means that human beings are the authors of their actions. ${ }^{93}$

The third principle, promise and threat, emphasized that the concepts of divine "reward" and "punishment" agree with the proposed vision of the divine justice and human freedom; one of the requirements of freedom is that man can be mistaken and can be right, and one of the requirements of divine justice is the implementation of the promise and threat. Perhaps this principle, with the next, is the reason for rejecting of intercession by Mu'tazilah. ${ }^{94}$

The fourth principle, the intermediate position, relates to the answer to the common conflict about the status of the sinful Muslim (Murtakib al-Kabira), where the Mu'tazilah recommended a compromise position by which the sinner was not banished from the Islam fold (al-Manzila bayn alManzilatayn). ${ }^{95}$

The fifth and final principle is the only practical one; it asserted the duty of the Islamic community to demand what is 
right and condemn what is wrong. The Mu'tazilah engaged in this principle practically within and outside the Muslim community. Because of the central importance of the first two theses (or principles) the Mu'tazilah came to be known as the upholders of justice and monotheism (ahl al-adl wa alta whid).

As we notice, the five principles, rather than being apologetic or even out ward looking, clearly represent a bold and, as it turned out, a consequential attempt to define the meaning of Islamic monotheism and an earnest effort to come to grips with what the Mu'tazilah conceived as inevitable moral and political implications. Indeed, al-kalam, whether that of the Mu'tazilah or of the later, "orthodox", Ash'arites, declares itself as a from of genuine knowledge (ilm), against the passive acceptance of authority in matters of faith, an attitude which it calls by the name of taqlid (the imitation or unquestioning following of authority), and which it seeks, expressly and as a matter of principle, to replace by a state of knowledge (ilm) rooted in reason (aqI). The Ash'arites radically modified the Mu'tazilah view, as they tended do which other Mu'tazilite doctrines; but they retained the commitment to rationally acquired knowledge as the goal of their endeavour. As the fourteenth - century Ash'arite "Adud al-Din al-Iji" (1281 - 1355) put in an unequivocal terms, the first advantage of kalam was to raise the adept «from the perigee of taqlid to the apogee of certainty», where 'certainty' (iqan) refers to a state of consciousness acquired by rational argument (nazar) and confirmed by tradition. And the Ash'arites theory of knowledge remained a cornerstone of their system. ${ }^{96}$

In fact, a quick view to the development of the views of the sects of al-Kalam, shows us clearly the neutrosophic feature of ideas, a feature under which the idea adopts, as a first step, the properties of the value. The first property of the values is the polarization; namely the existence of the tense opposition between two poles; in logic, there is the opposition between: 
"right - wrong", "true - false", "valid -invalid"; in religion: "faith - atheism", "obedience - disobedience", "lawful forbidden"; in ethics: "good - evil", "happiness - misery", "pleasure - pain"; in aesthetics: "beauty - ugliness"; and in law: "legal - illegal", ... etc. In the light of that polarization, we can understand the violent tension that accompanied the emergence of the sects of al-kalam, which peaked in the third century A.H., it was truly the age of polarization among the systems of Muslims and their sects: between jurists and Mystics, Mystics and Hanabilites, Hanabilites and Mu'tazilah, Mu'tazilah and Anthropomorphists (especially from Shi'a), Shi'a and Ahl al-Sunnah (the folks of tradition), Ahl al-Sunnah and Kharijites, Kharijites and Murji'ah, ... etc. For example, the polarization reached to the peak of intensity between Mu'tazilah and Hanabilites at the time of Ibn Hanbal's ordeal [from the era of caliph Al-Ma'mun to the era of caliph Al-Wathiq (d. 847)]; the ordeal that bequeathed an anti-current to Mu'tazilah since the caliphate of AlMutawakkil (d. 861), peaked at the time of caliph Al-Qadir (d - 1031), who prohibited from talking and teaching according to the Mu'tazili system, and warned the violator with penalty. Another example of the violent polarization, known as "the ordeal of Ghulam al-Khalip' (= the ordeal of the servant of Hebron), or rather the ordeal of Mystics from Hanabilites. Ghulam al-Khalil was Hanbali, and he accused the Hanabilites of atheism (Zandaqa) and provoked the masses to them; this has led the caliph "al-Muwaffaq" $(d-891)$ to order the arrest of a large number of Mystics who reached 70 odds. The peak of intensity between jurists and Mystics was with the temptation of Mansur al-Hallaj (858 - 922), which the fatwa to kill him has been issued in $910 .{ }^{97}$

If these ordeals and strifes entirely are unfortunate matters in the conflict among ideas, the intensity of polarization, on the other hand, have led to the severity of thought and its rebellion, and caused the overflow of energy in every system that presented the culmination of its ideas and the most 
geniuses of its scholars and thinkers, who did not have counterparts in the subsequent centuries.

It was natural after that the ideas lend to moderation; in mysticism, most of mystics gave the blame on al-Hallaj due to his reprehensible innovations (vagaries), and the mysticism after him tended to the idea that it is necessary the commitment to the truth by Shariah, and the exclusion of vagaries. The moderation reached to its completion in the $5^{\text {th }}$ century at the hands of al-Ghazali. In Ilm al-Kalam, the people alienated from the Mu'tazilah, not because of the transition of state from them, but for their role in the ordeal of Hanabilites on the one hand, and for their rational opinions that are not digested by the minds of the public on the other. It was expected to prevail the Hanabilites, but after their leader, they raised the discontent of the people, because they were very extremists on violators, whether from the other jurisprudential doctrines (while their leader was not), or from the doctrines of kalam or mysticism.

No coincidence then - but it is a natural evolution of ideas that three accompanied schools arise in the fourth century A.H., all of them are seeking the central solutions between Mu'tazilah and Hanabilites, or between the text and the mind. These schools are al-Taha wiyyah (a reference to Abi Ja'far alTahawi - d. 933) in Egypt, al-Ash'arites (a reference to Abi al-Hasan al-Ash'ari - d. 935) in Iraq, and al-Maturridiyyah (a reference to Abi Mansur al-Maturidi - d. 944) in Samarkand and beyond the river, to start thinking with new features. ${ }^{98}$

\section{Ilm Usul al-Fiqh (the Study of the Origins, Sources, and Principles of Islamic Jurisprudence)}

Jurisprudence (fiqh) in the Arabic language is the knowledge of something and the understanding of it. It is said: «So - and - so has a jurisprudence of the good and the evil», that is he knows and understands them. ${ }^{99}$ But in the idioms of jurists it is the knowledge of the legal practical judgments that 
are acquired from their detailed evidences, or it these judgments themselves. ${ }^{100}$

It is intended here with the judgments what are proved to be the acts of charged; such as obligatory, recommended, unlawful or prohibited, reprehensible or not recommended, permissible or allowed, aside from the fact that such acts are valid or invalid. ${ }^{101}$

The judgments were restricted as legitimate to show that they are attributed to religion, i.e., they are taken from it directly and without any intermediate; then the definition do not contain the mental judgments (such as the knowledge that the whole is more than the part, and the one is the half of the two, and the world is accidental), nor the sensual judgments (such as the knowledge that the poison kills), nor the positive judgments (such as the knowledge of any grammatical rule). ${ }^{102}$

Also, it is required in these legal judgments that they are "practical", they does not include the doctrinal or ethical judgments, which are meant by al-Kalam and ethics. They also require that they are acquired from the detailed evidences by reasoning and intellectual activity. The detailed evidences are the partial ones that each of them relates to a particular issue and provided by a certain judgment. ${ }^{103}$

So, ilm usul al-fiqh is regarded as the method of jurist and his logic in front of the method of philosopher and his logic.

The first seed of ilm usul al-fiqh was sown by the hands of Sahaba (the companions of the Prophet); they had to make effort to devise the judgments which are not in the Quran and Sunnah, but they did not feel the need to put methodical rules for effort (ijtihad) or the routes of reasoning and deduction, that because of their knowledge of the Arabic language and its semantics and syntax, as well as their knowledge of the reasons of revelation (Quran and Sunnah) and the secrets and wisdom of the legislation, where they were in the company of the Prophet or close by. However, after the age of Sahaba and the closest of their followers, and the spread of Islamic 
countries, many and various facts and events happened; the Arabs blended with non-Arabs so that the Arabic language no longer with its first safety; the diligence and diligent people became many; their routes in deduction became numerous; the debate and controversy expanded; the suspicions and possibilities increased, ... etc. The result was that the need become urgent to develop rules and principles and controls for the ijtihad, to which the diligent people (al-Mujtahidun) can turn in case of disagreement, and to be balances of the jurisprudence and the right opinion. It is common among scholars that the Imam Muhammad Ibn Idris al-Shafi'i (767 820) had been the first to record this science and wrote in it separately, then the jurists succeed one another after him to write and arrange the researches of this science, as well as they expanded it and added to it. ${ }^{104}$

We have known in Islam several Madhhabs (schools of jurisprudence) which are different from each other, but their difference is not a division of the Islamic religion; i.e., they are not substitute religions, but they are faces to interpret and understand the body of Islamic law (shariah), they are windows to look on shariah, and methods to the research, study and understanding; or scientific manners in the deduction. They all aspire reaching to the knowledge of what God revealed and legislated. The most famous of these schools:

- Hanafitism; the founder is Imam Abu Hanifa (696 - 767).

- Malikitism; named after Imam Malik Ibn Anas(715 - 796).

- Shafi'itism, named after the Imam al-Shafi'i.

- Hanbalitism, named after the Imam Ibn Hanbal.

- Jaafritism (tracking Shi'ite sect), named after Imam Ja'far al-Sadiq $(703-765)$.

Most of these schools, as seen from the above definition of jurisprudence, looking at five types of the judgments of charging; namely: 
1. Prescribed or obligatory (al-Wajib or al-Fard), which relates to the required (lazim) or mandatory (muhattam) act religiously; such as performance of prayer, giving of alms, charity of parents, fulfillment of contracts, and so on. It is an act that who leaves it is censured and punished, while who does it is eulogized and rewarded.

2. Recommended or preferable (al-Mandub or al-Mustahab), which is within the scope of virtue or meritorious (alFadila); such us the writing of debt, marriage, imitation of the Prophet in his habitual matters that issued from him like the etiquette of eating, drinking and sleeping. All these are acts that who does them is eulogized and rewarded, while who leaves them is not censured and punished.

3. Unlawful (al-Haram or al-Mahzur), or the act that prohibited by Islam as inevitable and compulsory; such as adultery, drinking the wine, gambling ...etc. Who does these acts is a sinner and disobedient, and who leaves them is an obedient.

4. Reprehensible or not recommended (al-Makruh); it contains what the abandonment of it is better than doing it, so that who does it do not be a sinner, even though he is blamed, and who leaves it is eulogized and rewarded if he leaves it in the cause of Allah; such us divorce, wasting money, and eating the flesh of horses.

5. Permissible or allowed (al-Mubah); it relates to what the God and His messenger let us choose between leave or do it; it also called the lawful (al-Halal); such us the eating, innocent recreation, and matrimony, ..., provided that does not leave whole or reach to the extent of wasteful.

As for the legal sources of the deduction of judgments, or so-called the evidences of judgments, they are three types:

1. What is in a place of an agreement by the leaders of Islam (Imams): this type contains the Quran and Sunnah.

2. What is approved by the crowd of Muslims; it includes: 
- Unanimity or consensus (al-Ijma); it means the agreement of Muslim jurists, in any age, to a legal judgment, after the death of the Prophet.

- Measurement (al-Qiyas); it is the process of analogical reasoning from a known injunction to a new injunction, because there is a common cause between them.

Al-Nazzam (d - 840), from al-Mu'tazilah, rejected both ijma and qiyas; some kharijites rejected ijma; some Jaafrites and Ahl al-Zahir (or al-Zahiriyah) ${ }^{105}$ rejected qiyas.

3. What is the subject of a disagreement among jurists, including their majority who said with qiyas. This type contains:

- The admiration (al-Istihsan); it is the abandonment of an obvious qiyas for a hidden one, because the latter is stronger than the first in its argument, or because there is a legal evidence relating to it.

- The free benefit (al-Maslaha al-Mursalah); it brings a beneficial interest of the Islamic nation, or pushes a damage from it, and which there is no a judgment concerns it in the Quran or Sunnah.

- The prevention of instruments (Sadd al-Dhrai'i); i.e., the prevention of mediums that can be led to evil.

- The custom (al-Urf); which is the community marched and got used to it in its life, whether it was a saying or deed, so that it does not contrary to a text of the shariah, and does not fail a considered interest, nor brings a preponderant harm.

- The saying of Prophet's companions (Qawl al-Sahabi); namely, tacking the sayings of the companions if the judgment abstained in the Quran, Sunnah, and ijma. If the companions are different, we can compare among their words.

- The law of previous nations (Shar'a mann qablana); i.e., the judgments that legislated by the God for who preceded 
us of the nations; and that the God revealed to His messengers and Prophets to report to those nations, provided that the Quran contains them or the Sunnah includes them.

- The continuation (al-Istisihab); it means the survival of any matter as it was unless there is change. There are rules and principles based on this evidence; the most important of them that the origin in judgments is the permission, except if there is a text to the prohibition.

We will discuss in chapter three of this book the evidence, or origin, of syllogism in detail as it is the most field of the intellectual creativity for Muslims which is marked by fertility, and because Muslims approach with it to the systems of contemporary logic, i.e., many-valued logic, especially the neutrosophic logic

\section{E. Sociology or Philosophy of Politics or Philosophy of History}

As Muslims excelled in the mathematical, natural, religious sciences and their philosophy, they excelled also in sociology, politics, history, and their psychological, economic, and moral aspects, as well as the philosophical theorizing of them. These are all overlapping investigations in Islamic thought; meaning that they lead to each other and connected with each other closely in most of the writings of thinkers of Islam. Regarding this perspective, we can refer, for example, to al-Farabi, who developed in the science of politics three main books, by which he is regarded the founder of political philosophy for the Muslims; they are: ${ }^{106}$

- The book of attainment of happiness (Kitab tahsil alSaadah).

- The book of public administration (Kitab al-Siyasah alMadinyah).

- The opinions of people of virtuous city (Ara' ahl alMadina al-Fadila). 
In these books, al-Farabi seeks to understand the elements and intricacies of sociology and anthropology; and to study the characteristics, attributes, advantages and bases of society. He concludes that the only way to attain the human perfection is only through the phenomenon of society and its changing laws according to the change of conditions and countries. We can also refer to the jurist al-Mawardi $(\mathrm{d}-1058)$ and his theory in the leadership (al-Imama), which contained in his important book "The ordinances of government" (al-Ahkam al-Sultania wa al-Wilayat al-Diniyya), in which he rely mainly on the jurisprudential grounds to the installation of the leader (Imam), his functions, and his relationship with the people. Thus, it is not possible to understand his theory but through the Islamic jurisprudence and its developments that imposed by the flowing movement of the history and society of Muslims.

The highlight of examples that I can mention in this subject is the impressive book of Ibn Khaldun, titled "The introduction" (al-Muqaddimah), by which he developed the foundations of the philosophy of history, and adopted the principle known as the integration of the factors in the interpretation, for he was not explain the phenomena of social or historical facts from a single view relies on a specific aspect with the neglect of other aspects, but we find in his analyses a synergy between the economic interpretations and the psychological interpretations, as well as the geographical and political visions; therefore, researchers disagree about the classification of his Introduction, and various schools of thought dispute about him; a group considers him the founder of sociology, another group regards him as the motive and founder of the philosophy of history; a third group calls him one of the first parents of economy; and a fourth group argues that he is the first theoretician of the geopolitics.

In addition, Ibn Khaldun possessed alone a special method in research, by which he collected between the category of universal proposition and the category of justification (the two 
main categories of the philosophy of history), and between the category of dynamism and the category of dialectic; although he admitted the unity of human nature, he saw that it is necessary to take into account the differences of circumstances from a human society to another. This can not be explained but by the dialectical movement of ideas and factors that govern the course of history, I mean that the internal contradiction in which the idea and its opposite meet, or in which one factor lead to conflicting or contradictory results. We will show that in detail in the subsequent pages.

\section{F. The Philosophy of Grammar}

The language in itself was the backbone of Islamic civilization and its favourite axis, By Arabic language, as the language of Quran and the uterus combined its meanings and its rules of judgments, Islam moved from the local context to the global context. It is spoken by Arabs and non-Arabs, and the solecism, or grammatical mistake (Lahn), crept into it, and the various words and dialects misted it. Then the need become urgent to establish rules for conservation and protection of the Arabic language, hence it was the grammar and it was the linguistics.

It was not strange that the grammar clashes with logic after translation of the Aristotelian works into Arabic; the word chosen by the translators of Aristotle to the indication of this science, i.e., the logic (= speech (Mantiq), is the same as that used sometimes to refer to the grammatical and linguistic researches and classifications, this has been sufficient alone to cause the conflict and controversy between the grammarians on the one hand, and the logicians on the other, on the superiority of one over the other science, especially in the midst of a wave of religious skepticism that accompanied the early transfer of intellectual production of Greek into Arabic, and the range of its compatibility with the Islamic principles and faiths. 
Perhaps the most remarkable scene of this dispute is that the famous debate which is conveyed to us by Abu Hayyan alTawhidi (930 - 1023) in his book: «Enjoyment and conviviality (al-Imta wa al-Mu'anasa), and which took place in Baghdad during the $10^{\text {th }}$ century between al-Sirafi (d. 978), who was a linguist, grammarian, jurist and speaker, and Matta Ibn Yunus al-Qinai (d. 940), whose presidency of logic in his time ended to him».

It is known that the debate had ended to the victory of grammarian and the defeat of logician for many reasons, including that Matta fell into two big mistakes, namely that the logician do not need to be acquainted with language and grammar, and the grammar researches in the pronunciation only, not in the meaning; as shown in his response to al-Sirafi when the latter asked him about the uses and rules of one of the Arabic letters, where he said: «this is a grammar, which I did not research in it, while the grammarian needs seriously to logic, because the logic researches in pronunciation. If the logician goes by the pronunciation, that is by accident, and if the grammarian goes by the meaning, that also is by accident. But the meaning is nobler than the pronunciation, and the pronunciation is humbler than the meaning». ${ }^{107}$

One of the reasons also that al-Sirafi was not only a leader in the Arabic language and its rules of grammar, but he was also a skilled in logic without says that, as well as he was a superior in the dialectic and the art of controversy to the degree of fallacy sometimes; he addressed to his opponent linguistic questions, which he was ignorant of their aspects, then fell into the confusion and disturbance. This means that al-Sirafi was aware of the need to connect the grammar with the logic, and the logic with the grammar, taking in mind that the grammar is a specific science, and its rules differ from one language to another, which he expressed by saying: «The grammar is a logic, but it is skinned from the Arabic language, and the logic is a grammar, but it is understood by the language; the difference between pronunciation and meaning 
is that the pronunciation is natural, while the meaning is mental, so the pronunciation is extinct during the time, because the time follows the track of nature to replace it with another track; so the meaning is fixed during the time, because the meaning is produced by the mind ..., however, the meanings are not Greek or Indian, as the languages are Persian, Arabic and Turkish; nevertheless, O Matta, you claims that the meanings are acquired by the mind, investigation and thought, but you seems to lack the rules of Arabic language, then how are you underestimate the Arabic at the same time you explain the Aristotle's books in it, with your ignorance of its truth?.

On the other hand, Abu Nasr al-Farabi, at one time a student of Matta, was the first Arab logician to take seriously the questions of the relation of logic to grammar and of language to thought. His "Enumeration of the Sciences" (Ihs'a al-Ulum) briefly formulates the idea of logic as universal grammar: «grammar furnishes the rules proper to the utterances of a given language; logic furnishes the rules common to the utterances of all languages». As well as he tried to convince other Muslims of the usefulness of Greek logic by illustrating Aristotelian forms of inference in terms taken from Islamic theology and jurisprudence. He thus preceded al-Ghazali in formulating the kind of argument that finally succeeded in securing for Aristotelian logic a permanent place in Muslim education. ${ }^{109}$

Al-Mutakallimun (Muslim theologians) also contributed serious and innovative studies in the problem of meaning, with which they anticipated the leaders of the philosophy of language in the modern age; for example, it is commonly said that thanks to distinguish between linguistic meaning of the name and its reference, is due to the German philosopher and mathematician "Gottlob Frege" (1848 - 1925), especially in his article published in 1892 under the title "On sense and meaning", ${ }^{110}$ but we find clear roots of this distinction at both Mu'tazilah and Ash'arites several centuries before the 
publication of the above - mentioned article, it is what we find, for example, at Abu al-Qasim al-Zamakhshari (1074 1143 ) in his "The detailed" (Kitab al-Mufassal), and Fakhr alDin al-Razi (1149 - 1209) in his "Eastern studies in metaphysics and physics" (al-Mabahith al-Mashriqiyya fi ilm al-Ilahiyyat wa al-Tabi'iyyat).

The nature of problems of kalam imposed on alMutakallimun to talk about language grammatically, logically, and philosophically, and the problem of God's speech was the axis of the problem of meaning; al-Mu'tazilah considered that the God is Eternal whereas His speech is contingent - Moses heard Hem towards the tree - Hence the name, i.e., the Speaker, is unlike the Essence divine, and the name linked to the contingent speech, while the Essence (God) is Eternal. However, al-Ash'arites describes the God's speech as Eternal; they intend the psycho-speech, then the speaker truly is the God, hence, the name (God) is the Essence (the speaker). Each sect of them states the arguments in support of its point to, the contemporary philosophical analyses in semantics, such as, for example, their argument that the name may be existing without any reference; or their emphasis that the names may be many though the reference is one; such as the synonymous names, or that the name may be one though the references are many, such as the common names, etc.

In fact, what we presented of the types of philosophical cultures in Islam, and what we gave as examples of each, if they show anything, they show that Muslims had a particularly thinking, in which they expressed themselves. What we'll concern in the following pages of this book is to describe the neutrosophic feature of this thinking; namely its logic and method that has characterized it and granted it all this wealth. 


\section{Notes of Chapter 2}

1. This chapter is written by Salah Osman.

2. See Sabra, A.I., Situating Arabic Science: Locality versus Essence, Isis, 87, 1996, p. 654.

And see also Tawfiq al-Tawil, On our Arab-Islamic Heritage (Fi Turathina al-Arabi al-Islami), Series of the world of knowledge, the National Council for Culture, Arts and Literatures, Kuwait, No. 87, March 1985, p.7.

3. Bartold, V. V., The History of Islamic Civilization, (Tarikh al-Hadara al-Islamiyah), Trans. into Arabic by Hamza Tahir, fifth edition, The house of knowledge (Dar al Ma'arif), Cairo, 1983, pp. 35 - 37.

4. Ibid, p. 39.

5. Ahmad Mahmoud Subhi, On the Philosophy of History (Fi Falsaft al-Tarikh), The Establishment of University Culture (Mu'asast al-Thaqafa al-Jami'iyah), Alexandria, 1975 , p. 245 \& p. 261.

6. "Herodianism" is a name of an approach adopted by one of the ancient sects of Jews (Herodians). The name is derived from "Herod Antipns", who was appointed by Roman in 47 B.C. as a governor of Galilee, then he wanted to curry favor with Julius Caesar on the one hand, and to appease Jews on the other. He rebuilt the temple, but not satisfied by the hard-liners of Jews because he constructed a theater and stadium in Jerusalem. Thus the meaning of the name had become associated with the concept of morphosis. The use of this term is often associated with another term; i.e., the Pharisees, which is the name of an ancient Jewish sect known with its affectation of the piety.

7. "Pseudo-morphosis" is a term used by Spengler as a 
description of the situation, in which a deep-rooted civilization that has lost its strength is forced to submit to another dominant civilization, and to take over some of the outward forms and the learning of this more advanced civilization. Therefore, the viewer to the surface thinks that the defeated civilization had disappeared, while it is dormant behind the foreign rind imposed upon it.

8. The secular is one who concerns himself with the affairs of world alone without religion. He is unlike the clergyman who is interested in the affairs of religion alone (in the Western Christian society). Then, we can describe the secularism as a worldly or non-religious movement that calls to the separation of state and religion. The verbal derivation of the word "Secularism", in English, due to the Latin word "Saeculum", which means the world or the earth. The term was used for the first time with the signing of the Peace of Westphalie in Germany in 1648, which put an end to the conflicts and religious wars in Europe due to the control of the Church to the state and all aspects of life. If the separation of religion and the state has saved the Christian West from the severity of clergy and their dogmatic attitude about life and the results of science that are assessed as a "religious heresy", the issue with its European specification is not thinkable in the Muslim community. That because Islam is the total science that embraces all types of partial sciences and provides them with the healthy climate that adequate to the living on earth and the deputation on it. Islam is also the religion that explicitly invites to pay attention to the life as if it is permanent and do not stop by the death; as well pay attention to the afterlife as if it no more than two bows length or even closer. This was expressed by the Quranic verse:

And betake yourself to Providence for the grace He made to abound in you seeking His satisfaction - to 
ensure spiritual relish - Here after, and do not neglect your share of worldly enjoyment of what is lawful. And be kindly disposed and benevolent to others as Allah has been to you and do not pursue mischief nor create discord on earth. Allah detests those who have ill - will and mischief is natural to them

$$
(\text { Al-Qasas }=\text { The Narratives, verse 77) }
$$

Then, we can describe the Muslim secularists as people who do not understand the truth of religious, but they are captured by the achievements of Western civilization. Therefore, they imagine it as the only civilization of age, and say that those who want to qualify must seek to catch up with it and melt in it without paying attention to the ancestral heritage coated with the issues and teachings of religious!.

9. As a direct result of the attempt of Mustafa Kemal (Atatürk) (1881 - 1938) to make the culture of Turkey as a part of the European culture, the contemporary Turkish suffers as he changed his life totally, and severed the link with his past. The writing of the Turkish language in Latin letters made the modern Turkish youth unable to read their intellectual heritage whether in the old Turkish, Persian or Arabic. Atatürk was not content with the change in the economic or political field, but the movement of morphosis swept away all fields and turned the life of the Turkish people upside down in all faces of the activity. Swiss law has been adopted in the judiciary instead of Islamic law; the young wore the hat to prevent them from full genuflection in prayer.

The herodianists have tried to make their home a Western nation and a Western country, but the Turkish become now a character who is not Eastern or Western. Although Turkey is, politically, a powerful ally to the Western countries, the latter do not consider Turkey a part of their civilization, and the Turkish become addressing 
the European with words from his Gospel: «We have piped unto you, and ye have not danced; we have mourned unto you, and ye have not lamented» (Matt, 11: 17). See Ahmad Mahmoud Subhi, On the Philosophy of History, p. 287. (It is noteworthy, as we have mentioned in the introduction, that Smarandache has lived for two years in a camp for political refugee in Turkey. Thus, Turkey has been embraced the Westerns who now try to prevent it from entering the European Union!).

10. "Zealotism", is a name of an ancient extremist Jewish movement. The word "Zealot" in Hebrew refers to a person who is zealous; one who is full of zeal for his own specific beliefs or objectives, usually in the negative sense of being too passionate; a fanatic. In the modern English the word is used to refer to any from of extremism, jealousy or excessive zeal.

11. Quoted from Muhammad Shama, Islam in the Western Thought (al-Islam fi al-Fikr al-Gharbi), Wahba Library (Maktabt Wahba), Cairo, 1980, p. 28.

12. Muhammad Ghallab, Some of the Treasures of Islam (Menn Kounuz al-Islam), p.15, quoted from Husni Ahmad Al-Saiyed Hammad, Arab Civilization: its Inception, its Evolution, its Effects (Al-Hadarrah al-Arabiyah: Nash'atiha, Tatwuriha, Atharaha), The Establishment of Compilation and Publication \& The House of Arab writer (Mu'asast al-Ta'alif wa al-Nashr \& Dar al-Katib alArabi), Cairo, 1967, p. 18.

13. Ahmad Amin, Dawn of Islam (Fagr al-Islam), The General Egyptian Book Organization (Al-Hay'ah alMisriyah al-A'ma li al-Kitab), Cairo, 1996, p.58, and see also Ibn Khaldun's Introduction, ed. by Ali Abd al-Wahed Wafi, Passim.

14. Le Bon, Gustave, The Civilization of Arab (Hadarat alArab), pp. 96 - 97 \& p.89, quoted from Husni Ahmad, OP. Cit., p.19.

15. Quoted from Husni Ahmad, Ibid, p. 21. 
16. Ahmad Amin, Dawn of Islam, pp.59-60.

17. See Ali Abu Milhem, Arab Philosophy: Problems and Solutions (Al-Falsafa al-Arabiyah: Mushkilat wa Hulul), Izz al-Din Establishment for Printing and Publishing (Mu'asast Izz al-Din li al-Tiba'ah wa al-Nashr), Beirut, 1994, p. 5.

18. Ali Sami Al-Nashar, The Emergence of Philosoph- ical Thought in Islam (Nash'at al-Fikr al-Falsafi fi al-Islam), eighth edition, The House of Knowledge (Dar alMa'arif), Cairo, 1981, p. 56.

19. Brown, E. G., Arab Medicine (Al-Teb al-Arabi), Trans. into Arabic by Ahmad Shawki, reviewed by Muhammad Abd al-Halim al-Oqbi, The Establishment of record of Arab (Mu'asast Sijil al-Arab), Cairo,1966, pp. 22-24.

20. Bartold, V. V., The History of Islamic Civilization, OP. Cit, pp. $62-63$.

21. Ibid, pp. $68-69$.

22. Ibid, pp. $72-73$.

23. Hamza Tahir, the Arabic translation of Bartold's The History of Islamic Civilization, p.73n.

24. See for more detail, Sharif al-Chaoubachi: Long Live the Arab Language, Down Sibawayh (Tahiya al-Lugha alArabiyah, Yasqut Sibawayh), The General Egyptian Book Organization (Al-Hay'ah al-Misriyah al-Ama li al-Kitab), Cairo, 2004), Chapter IV, entitled: Is the Arabic Language Sacred?.

25. Compare, for example, what was established in the West's conscience, and what is aired by the media in the United States and Europe, of erroneous views and false beliefs about Islam and its adherents, where the Muslim is described as retarded and barbaric and terrorist, ... etc. This without extrapolation of the real principles of Islam or of the data of Arab-Islamic reality in the light of the political and economic practices that pour in the category of Western interests, indeed, without the slightest regard for the culture of others and their legitimate human rights. 
It is not invisible that this influence of the media did not stop only at the limits of the ordinary citizen in the West, but it has also extended to its leaders and thinkers, as shown in the argument of President George Bush after the events of September $11^{\text {th }}$, when he announced clearly that he was waging a sacred crusade against the Islamic terrorism!.

26. See Bronowski, Jacob, The Ascent of Man (Irtiqa al-Insan) Trans. into Arabic by Ahmed Mostajir as The Civilizational Development of Man (Al-Tatwur al-hadari li al-Insan), The General Egyptian Book Organization (AlHay'ah al-Misriyah al-Ama li al-Kitab), Cairos, 1997, p.133, also Kessler, John, Giordano Bruno: the Forgotten Philosopher,

http://www.infidels.org/library/historical/john_kessler/gior dano_bruno.html

27. The eugenics is a movement with political and social goals; it had immoral consequences that labeled it bad reputation, even after it developed into a stand-alone science in our modern world; a science that is working to locate thousands of the pathological genes in human and subject them to accurate diagnoses augur well for access to new types of medical treatment for diseases, the hope of overcoming them was weak until recently. This movement started in Britain with a program of the human proliferation developed by the British physiologist and anthropologist Francis Galton (1822 - 1911); he called it eugenics in 1882, a word of Greek origin that means wellborn or pureblood. The aim of the program was to stop the decay and deterioration presumed in the human gene stock, and to improve the physical and intellectual qualities of the future generations in accordance with the objective assessment of their value. In other words, Galton wanted to sharpen the blade of natural selection, according to Darwin' theory (1809-1882) in the biological evolution, after it blunted by our excessive humanity. It was natural 
that these ideas lead to tragic and horrific results; in Britain, the program of eugenics are applied on the lower and middle classes in society, fearing that the booming population of the working class with poor genes, hence, the deterioration of the genealogical tree of deep-rooted families of the British, so it was the exclusion of those who have weak hereditary dispositions; physical, mental or professional, either by sending them to the battlefields, or by subjecting them to the forced sterilization. In the USA, about 30 American states, between 1907 - 1930, endorsed laws allowing forced sterilization of the criminals and the mentally ill people; with the mid-1930 was about 20.000 Americans had been subjects to forced sterilization against their will, in order to get rid of their lowly genes. On the other hand, there was unanimity among the American scientists of eugenics on the gravity of the successive waves of immigration from European countries - Eastern and Western - for ethnic purity of the first migrants, which prompted the Congress in 1924 to adopt a notorious resolution that restricting immigration to the US. In Germany, we know too will the depth to which Nazism snak with its applications of the ideas of eugenics.

See Cartwright, John, Evolution and Human Behavior: Darwinism Perspectives on Human Nature, Macmillan Press, LTD, London, 2000, pp. 322 FF, also Salah Osman, Darwinism and Human: the Theory of Evolution from Science to Globalization (Al-Darwiniyah wa al-Insan: Nazariyt al-Tatwur menn al-Ilm ila al-Awlama), Establishment of Knowledge (Munsha't al-Ma'arif), Alexandria, Egypt, 2001, pp. 143 FF.

28. The fact is that the racism has tended to biology to solicit the scientific support, even before the emergence of evolutionary thinking during the $1800 \mathrm{~s}$, the racists, especially in the USA, have used a combination of biology and religion to justify their exploitation of Africans as a low race. It was a bad theology and a bad biology!. After 
the emergence of Darwinism, the racism is set up on a new basis, and of course the result is the same as long as it is governed by a prior ideology that wishes to see some races higher or more sophisticated than other races. This view has encroached into medicine. Tack, for example, The Dawn's Syndrome; a problem caused by a mistake in chromosomal genetics lead to the birth of a child with a weakness of the mind and a broad flattened head and face and sloping eyes. Its Victorian founder J.L. Dawn (18281896) did not find a name for it better than Mongolism, considering that those who are suffering from this disease resemble a race lower than the European race, i.e., the Mongolian race (Cartwright, p. 334).

As in the fields of history and anthropology, as Toynbee stated, some Western historians have embraced the racial view in their historical researches, the view that consider the Nordic race, with the white skin, the yellow hair, and the blue eyes, or what is called by Nietzsche the blond monster, is the highest race. Toynbee criticizes this view as a historian; he limits the civilizations that the Nordic contributed to build them, and those that others contributed to build them, indicating that most races have contributed to the establishment of the civilizations, as well as the fact that there are elements of white people did not contribute to the establishment of any civilization. And if the Negroid race - the black race - did not contribute to a civilization, the spiritual and intellectual superiority is not linked to the color of skin, but it due to the fact that the opportunity is not yet ready or because of a lack of incentive.

In fact, as far as the anthropology, there are no superior or inferior races, but environmental and historical reasons. There are main civilizations in the Middle East, India, and South America that have reduced or vanished, whereas they were in some day the holder of intellectual heritage for their age. As the possibility that the raise and decline of these civilizations due to genetic reasons, it can not be the 
subject of reflection. If the white nations have been imposed their hegemony during the modern and contemporary era, the great breeds and their civilizations in Egypt and China continued to thousands of years, and we have not any idea at all about the race or ethnicity which will carry the torch of civilization after half a century from now!.

See Lewis, John, Man and Evolution, trans. into Arabic by Saleh Jawad Kadhim as The Man, that the Unique Creature (Al-Insan, Zalik al-Ka'in al-Farid), The General Egyptian Book Organization (Al-Hay'ah al-Misriyah alA'ma li al-Kitab), Cairo \& the House of Cultural affairs (Dar al-Shu'un al-Thaqafiyah), Baghdad,1986, pp. 215-16.

29. Ahmad Mahmoud Subhi, On the Science of Kalam: a Philosophical Study of the Opinions of Islamic sects in the Origins of Religion (Fi ilm al-Kalam: dirassah falsafiyah li ar'a al-Firq al-Islamiyah fi usul al-Din), The Establishment of University Culture (Mu'asast al-Thaqafa al-Jami'iyyah), fourth edition, Alexandria, 1982, Vol.1: AlMu'atazilah, p. 245 \& p. 261.

30. There are five pillars of Islam, in summary, they are:

- The testimony of faith (Shahadah): the declaration that there is none worthy of worship except Allah, and that Muhammad (PBUH) is His messenger (Shahadat ann la ilah ila Allah wa ann Muhammad rasulu Allah).

- Ritual prayer (Salat): establishing of the five daily prayers (Iqamat al-Salat).

- Obligatory (religious) alms-giving (Zakat): giving of the charity to the poor by able Muslims based on the wealth that he or she has accumulated (Ita'a al-Zakat).

- Fasting (Ramadan): refraining from eating, drinking, or engaging in sexual intercourse from dawn to sunset during the month of Ramadan, the ninth month in the Islamic lunar calendar (Sawm Ramadan). 
- The pilgrimage to Mecca (Hajj): this is done during the month of Zul Hajjah, and is compulsory once in a lifetime for one who has the ability to do it. If the Muslim is in ill health or in dept, he or she is not required to perform Hajj (Hajj al-Bayt li mann Istat'a Ilayhi Sabila)

31. The calculus of legacies or the science of legacies (Ilm alMawarith) is the jurisprudence of the division of legacies and the distribution of them to their beneficiaries, so as each heir or heiress get his share of the estate. The estate is all the money and property that leaves when one dies.

32. Sabra, OP.Cit, pp. $662-663$.

33. Zaki Naguib Mahmoud, The renewal of the Arab Thought (Tajdid al-Fikr al-Arabi), Sixth Edition, The House Of alShurouk (Dar al-Shurouk), Cairo, 1980, pp. 21 - 22.

34. See Ahmad Amin, Dawn of Islam, pp. 110 FF.

35. See Mohammed Ibn Abu Bakr al-Razi, The Chosen of Correction Dictionary, (Mukhtar al-Sahah), ed. by Mahmoud Khater, The House of Hadith (Dar al-Hadith), Cairo, without date, item Read (Qar'a), p. 526, also Arabic Language Academy, The Concise Dictionary (AlMu'ajam al-Wajiz), Prefaced by Ibrahim Bayoumi Madkour, Ministry of Education, Egyptian,Cairo,1990, item Read (Qar'a), pp.494-95.

36. Abd al-Karim Zidan, Summary of the Fundamentals of Jurisprudence (Al-Wajiz fi ilm usul al-Fiqh), The Islamic House of distribution and publishing (Dar al-Tawzi'i wa al-Nashr al-Islamiyah), Cairo, 1993, pp. 158-60.

37. Ahmad Amin, Dawn of Islam, pp. 116-20.

38. Ali Sami Al-Nashar, The Emergence of Philosophical Thought in Islam (Nash'at al-Fikr al-Falsafi fi al-Islam), OP. Cit., pp. $32-33$.

39. Ahmad Amin, Dawn of Islam, pp. 314 - 16.

40. See Abd al-Karim Zidan, OP. Cit., p. 165, and see for more detail :

- Mustafa al-Siba'i, Sunnah and its Place in Islamic legislation (Al-Sunnah wa Makanatiha fi al-Fiqh al- 
Islami), The Islamic Library for Printing and Publishing (Al-Maktabh al-Islami li al-Tiba'a wa al-Nashr), Beirut \& Damascus, 1978.

- Ahmad Ibn Hajar al-Asqalani, Picketing and Sunnah (AlI'itisam wa al-Sunnah), ed. by Khaled Abdel Fattah Shibl, The House of International Book \& The House of Egyptian Book (Dar al-Kitab al-Alami \& Dar al-Kitab alMisri), Cairo, 1990.

41. Some of these verses:

O you whose hearts have been touched with the divine hand: Obey Allah and be submissive to His will and obey the Messenger who is the only medium through whom Allah's guidance, command, ordinance and word are conveyed and expressed. And obey those among you who are invested with authority. Nonetheless, should you seem resolved to dispute any subject or a concern, then resort to Allah's Book -the Quran- and betake yourselves to the Messenger for aid if in fact your hearts have been impressed with image of religious and spiritual virtues and you are strongly disposed to believe that the Last Day is comfortable to reality. This is a wise course of action ensuring safety and leading to the intended purpose

$$
\text { (Al-Nisa }=\text { The Women, verse 59) }
$$

He who obeys the Messenger has in effect obeyed Allah, and he who turns a deaf ear must realize that We did not send you to watch over their innocence and folly

$$
(\text { Al-Nisa }=\text { The Women, verse } 80)
$$

And whatever the Messenger commands you to do, you must do, and whatever he forbids you to do, you do not do, and entertain the profound reverence dutiful to Allah; He punishes severely 


\section{(Al-Hashr $=$ The Thronging, verse 7)}

But never, by Allah, your Creator, shall they be recognized as Muslims and be sanctioned by all solemnities of religion until they have willingly accepted you as the judge and/or the arbitrator. and accepted your judgement and submitted to your authority with submissive reverence, and reasoned high of Providence

$$
(\text { Al-Nisa }=\text { The Women, verse 59) }
$$

And let it be clearly understood) that: No man or a woman whose hearts reflect the image of religious and spiritual virtues be in a position to exercise choice when once Allah and his Messenger have decided on a course of action, pronounced a Judgement or brought a cause to a decision. And he who disobeys Allah and His Messenger will have deviated from sense and will have cast himself head-long in the maze of error

$$
\text { (Al-Ahzab }=\text { The Confederates, verse 36) }
$$

42. See Ahmad Mahmoud Subhi, Between the Principles of Authentication and the Principles of Historio-graphy: a Comparative Study in Methodology (Bayn Usul al-Tahdith wa Usul al-Ta'arikh), in his On the Philosophy of History (Fi Falsaft al-Tarikh), OP. Cit., pp. $302-42$.

43. See Abu al-Qassim al-Shatibi, The Congruencies of the Sources of the Divine Law (Al-Muwafaqa fi Usul alShari'a), The Major Trading Library, (Al-Maktaba alTijariyya al-Kura), Cairo, 1975, pp. 4 FF.

44. For example, the Quran urged people to the request of science in many verses:

Ask them O Muhammad: «Do they compare, those who apply their minds' eyes and their hearts' ears to the acquisition of learning and by consequence they are well- informed and those who are ignorant of the facts! it is only those who exercise their intellect and lift to 


\section{Allah their inward sight}

(Al-Zumar $=$ The Throngs, verse 9)

Thus, such of Allah's servants as stand in awe of

\section{Him are the savants}

(Fater $=$ The Creator, verse 28)

This was confirmed by the prophet when he said: «Who move on a path looking for a science, Allah will make easy to him a path to the Paradise», \& «The superiority of scientist on the worshiper as the superiority of the uppermost on the lowermost among you». See Abu Zakariya Yahya al-Nawawi, The Meadows of Virtuous from the Messenger's Speech (Riyadd al-Saliheen menn Kalam Sayed al-Mursaleen), The Supplier Library (alMaktaba al-Imdadiyah), Mecca, without date, pp. $485-89$.

45. Abd al-Karim Zidan, Summary of the Fundamentals of Jurisprudence (Al-Wajiz fi ilm usul al-Fiqh), OP. Cit, p. 297, and see the full text of this hadith in The Meadows of Virtuous from the Messenger's Speech, pp. 6-7.

46. See: Fuad Muhammad Fakhr al-Din, The Future of Muslims (Mustaqbal al-Muslimeen), The Literatures of People (Matbu'at al-Sha'ab), Cairo, 1976, pp. $21-22$.

47. Ibid, p. 23.

48. Zaki Naguib Mahmoud, The Renewal of the Arab Thought (Tajdid al-Fikr al-Arabi), OP. Cit., p. 27.

49. Quoted from Ahmad Mahmoud Subhi, On the Philosophy of History (Fi Falsaft al-Tarikh), OP. Cit., p. 64.

50. Zaki Naguib Mahmoud, OP. Cit., p.48.

51. Ibid, p. 68.

52. See Ahmad Amin, Dawn of Islam, pp. 132 FF.

53. Zaki Naguib Mahmoud, OP. Cit., pp. 137 - 38.

54. See Osman, Salah, The Illusion of External World Between Language and Cognition (Wahm al-Alam al-Khariji bayna al-Lugha wa al-Idrak), The Establishment of Knowledge (Munsha't al-Ma'arif), Alexandria, Egypt, 2004, p. 29. 
55. Compare, for example, the story mentioned by the American Senator William Fulbright (1905-1995) in his «Arrogance of Power», about the missionary who was inviting people of Eskimos to convert to the new religion. After he returned from his mission spoke to one of his friends, saying: «You know, we stayed long years during which we can not do anything with the Eskimos, as they serve no sins. And we have had to teach them sin for a long period of time, before we can carry our message among them». Fulbright comments on this story, saying: «That reminds me of the story of the three scouts, who were notified by of their superior that the best thing they played in their day that they helped old woman in crossing the road. The superior said: this is great, but why so required all the three?, and they answered: because she did not want to cross the road»!.

See Fulbright, William, Arrogance of Power (Ghatrasat al-Quwa), trans. into Arabic by Mahmoud Shukri alAdawi, The House of Arab Writer for Printing and Publishing (Dar al-Katib al-Arabi li al-Tiba'a wa alNashr), Cairo, without date, p. 19.

56. Tawfiq al-Tawil, On our Arab-Islamic Heritage (Fi Turathina al-Arabi al-Islami), OP. Cit., p. 71 \& p. 76.

57. Sabra, Situating Arabic science, OP.Cit, pp.657-58.

58. See Tawfiq al-Tawil, OP. Cit., pp. $76-78$.

59. See Ahmad Mahmoud Subhi \& Muhammad Ahmad Abd al-Qadir, The Role of the Arabs in the Field of Sciences (Dur al-Arab fi Majal al-Ulum), The House of University Knowledge (Dar al-Ma'rifa al-Jam'iya), Alexandria, Egypt, 1985, pp. $426-33$.

60. The Twelvers (or al-Ithna-ashariyya) are one of the sects of Shi'a Imams, i.e., those who recognize the succession of Ali Ibn Abi Talib (Muhammad's cousin, son-in-law, the first young man to convert to Islam), and believe that Ali was appointed successor by Muhammad's direct order on many occasions, and that he is therefore the rightful leader 
of the Muslim faith pursuant to the Prophet's wishes. Their beliefs based on the idea of the Protected Leader. They were named Twelvers because they sequence their Imams to twelve, the first of them is Ali Ibn Abi Talib, and the latest, whose they are waiting for return, is Muhammad alMahdi Ibn al-Hasan al-Askari (b. 868). Among their distinguished personalities Abdullah Ibn Sab'a , a Jewish who alleged that he is a Muslim, and transferred what he has found in Judaism's thought to Islam, such as the nondeath and the return and others. It is known that the doctrine of Twelvers is the official doctrine of the State of Iran until now.

61. Ismailism is the second largest Shi'ite community after the Twelvers, it is affiliated to the Imam Ismail Ibn Jafar alSadiq (721-755). Its appearance is the support of Muhammad's family (Ahl al-Bayt), but its truth is a philosophical spirit, in a religious dress, based on the extravagant interpretations. The Isma'ili (al-Isma'iliyyun) tended to the strong immoderation and to the skepticism about the religious rites; for example, the Quran is symbols which are known only to knowledgeable people, and we must pass through the material screens to reach to the best case of the spirituality, so they are called also People of Interior. The sect branched into several sub-sects and still has the remnants in the Levant and India so far.

62. Zaydism is a sect of Shi'a. It is attributed to its founder Zayd Ibn Ali Ibn al-Hussuyn (698-740), who formulated a theory in politics and governance that he strove and was killed for the sake of. He was thinking that the leadership of Abu Bakr, Omar, and Othman (Allah well pleased with them) is valid. No one of the Fivers (Zaidiyya) had pronounced that one of Sahaba (the companions) is infidel. One of their doctrines is the permissibility of leadership of someone even though there is who is better than him. On the whole, they are the closer of Shiite sects to the Sunnis and the most moderate. Still present in Yemen so far. 
63. Gnosticism is a doctrine based on the conciliation between philosophy and religion, it is based on the idea of Emanation (i.e., the process of derivation or mode of origination, immediate or mediate, of the multiplicity of beings whether spiritual or material form the eternal source of all being, God, of whose being consequently they are a part and in Whose nature they somehow share). The doctrine includes a range of hidden views, in which we find a mixture of the Cabalistic teachings (the mystical symbolic ideas of Jews) and the Neo-Platonism, as well some Eastern teachings such as Mazdianism and Manicheism. The doctrine had an impact on philosophical thought in Christianity and Islam.

64. Kharijites is a general term embracing a variety of Islamic sects which, while initially accepting the caliphate of Ali Ibn Abi Talib (the fourth Caliph), later rejected him because he agreed to arbitration in its war against Muawiya Ibn Abi Sufyan (602-680). They said that the caliphate should be a free choice of Muslims, and acknowledged the validity of a caliphate of $A b u B a k r$ and Omar for the validity of their election, as well the validity of a caliphate of Othman in its beginning, but when he changed in the regime, they ruled that he must be abdicated from the caliphate. They acknowledged also the validity of a caliphate of Ali, but they said that he erred in arbitration and they ruled that he is infidel. They have multiple subsects, the best known :

- al-Azraqa, founded in Persia in 685 by Nafi Ibn al-Azraq.

- al-Najdat, founded by Najda Ibn A'mir al-Hanafi.

- al-Ibadiyyah, founded by Abdullah Ibn Ibadh al-Tamimi.

- al-Sufiryyah, founded by Ziyad Ibn al-Asfar.

65. Mu'tazilah is one of the sects of Kalam, especially with respect to the presentation of the subjects of Kalam in a full sectarian system. It represents the mental tendency in Islamic thought honestly. Its men were a section of the strongest advocates of Islam, intellectually and 
dialectically, against owners of other religions, as well as the unbelievers. This doctrine appeared in Basra, then another school appeared in Baghdad. These two schools combine the most important men in the Mu'tazilah and represent the two main trends of Mu'tazili theology.

66. Kaysani Shi'a is an extinct Islamic sect. Its members dissent of Shiism six years after the martyrdom of Imam Husayn bin Ali (626-680). They possessed alone their doctrines of the Shiites, and said that the leader (Imam) is Muhammad Ibn al-Hanafiya (637-710). Their name is attributed to Kaysan, who was the slave of Muhammad Ibn al-Hanafiya, or the slave of the Imam Ali.

67. Qaramita is a name of a religious and political movement that has grown from Ismailism. Its founder is Hamdan Qarmat (890-906) in the era of the caliph Abu al-Abbas Ahmad al- Mu'tadad (857-902). The sect adopted a carefully secret organization. One of the most important beliefs of it, that after Muhammad there are no Imams but only seven.

68. See Ali Sami Al-Nashar, The Emergence of Philosophical Thought in Islam (Nash'at al-Fikr al-Falsafi fi al-Islam), OP.Cit., pp. $224-226$.

69. Muhammad Al-Ghazali, A Defense of the Faith and Shari'a Against the Stabs of Orientalists (Dif'a an al-Aqida wa al Shari'a ded Mata'in al-Mustashriqin), fourth edition, The House of Modern Books (Dar al-Kutub alHadithah), Cairo, 1975, pp. $206-207$.

70. Ali Sami Al-Nashar, OP. Cit., pp. $47-48$.

71. Southern, R.W., Western Views of Islam in the Middle Ages, Harvard university press, Mass./ London, 1978, Quoted by Sabra, Situating Arabic science, OP.Cit, p. 657.

72. See: Sabra, The Appropriation and Subsequent, Naturalization of Greek Science in Medieval Islam: A Preliminary Statement, History of science, Volume 25, Part 3, Number 69, September 1987, p. 236. 
73. Abu Hamid al-Ghazali, Incoherence of the Philosophers, (Tahafut al-Falasifa), ed. by Sulyman Dunya, sixth edition, The House of Knowledge (Dar al-Ma'arif). Cairo, 1980, p. 76.

74. Ibid, p. 79.

75. Ibid, p. 80.

76. Ibid, p. 81.

77. Ibid, p.307.

78. See: Sabra, Avicenna on the Subject Matter of Logic, The Journal of Philosophy, Inc., 1980, p. 748.

And see also Abu Hamid al-Ghazali, The Chosen in The Science of the Origins of Jurisprudence (al-Mustasfa), second edition, The House of Scientific Books (Dar alKutub al-Ilmya), Beirut, 1983, pp.10FF.

79. Zaki Naguib Mahmoud, The renewal of the Arab Thought (Tajdid al-Fikr al-Arabi), OP. Cit., p.93, also al-Ghazali, Incoherence of the Philosophers, (Tahafut al-Falasifa), pp. $84-85$.

80. Sulyman Dunya, the introduction of the sixth edition of alGhazali's Incoherence of the Philosophers, p. 24.

81. Sabra, Science and philosophy in medieval Islamic theology: The evidence of the fourteenth century, Zeitschrift für geschichte der arabisch - Islamischen wissenschaften, Sonderdruch, Band 9, 1994, pp. 2-3 .

82. Ibid, p. 4.

83. Ali Sami Al-Nashar, OP. Cit., p. 47.

84. Abdel Rahman Badawi, The History of Islamic Mysticism from the Beginning Until the End of the Second Century (Tarikh al-Taswuf al-Islami: menn al-Bidaya hata Nihayit al-Qarn al-THani al-Hijri), The Agency of Literature (Wakalt al-Matbu'at), Kuwait, 1975, p. 18 \& p. 20, also Muhammad Mustafa Hilmi, The Spiritual Life in Islam (Al-Hayatu al-Ruhiyah fi al_Islam), The General Egyptian Book Organization (Al-Hay'ah al-Misriyah al-Ama li alKitab), Cairo, p. 116.

85. Abdel Rahman Badawi, OP. Cit., p. 21. 
86. Ali Sami Al-Nashar, OP. Cit., p. 53.

87. Abdel Rahman Badawi, OP. Cit., pp. 72 - 73.

88. Ibid, pp. $74-75 \&$ p. 81 .

89. Sabra, Science and philosophy in medieval Islamic theology, p. 5.

90. Mu'tazilah was not the first sect of Kalam, but there were other sects before it, such as al-Jahmiyyah and alQadiriyyah. However Mu'tazilah is the most important sect that presented the subjects of Kalam in a full doctrinal system, to such an extent that the discussion of the topics of Kalam became not go beyond the borders drawn by the men of Mu'tazilah.

See Ahmad Mahmoud Subhi, On the Science of Kalam, OP. Cit., p. 99.

91. Ibid, p. 115, also Sabra, OP.Cit, pp. 8 - 9.

92. Mu'tazilah insisted on their attitude for fear of the comparison between the Quran and the Christ, as both the Word of God; if the word of God is eternal, then the Christ is eternal, hence a deity, which is rejected by Islam. The Hanabilites have opposed this position for several reasons; the first is their opposition to the right of state to impose ideological doctrine of the people (as the Caliph alMa'mun has done with the doctrine of the creation of the Quran), the second is their opposition to the imposition of the subtle opinions of Kalam on the public. The third and most important reason is the connection in the minds of the public of the word «creation» (Khalq) with the word of « Invention» (Ikhtilaq), then the «lying» (al-Kadhib) on the one hand, and the connection of this word (i.e., «creation») with the meaning of the death on the other hand. For if every creature on earth and elsewhere the material and the immaterial, shall come to an end and go through the gates of death, then the Quran will be consumed, which is contrary with the Islamic faith that the Quran is preserved by Allah.

For more detail, see Ahmad Mahmoud Subhi, OP.Cit., 
pp. $126-137$.

93. Ibid, p.149, also Mahmoud al-Shafi'i, The introduction to the study of Kalam (Al-Madkhal ila Dirasit Ilm al-Kalam), second edition, Wahba Library (Maktabt Wahba), Cairo, 1991, p.89.

94. Also Mu'tazilah agreed unanimously (in harmony with their view of the divine justice and human freedom) on that it is not possible that Allah tortures children in the afterlife, because the God does not torture except who is deserving the torture as a result of his default, as for those who are not chargeable their deeds, whether they are children of Muslims or heathens, God is deemed far above the torture of them.

95. The sinful Muslim, or al-Fasiq, according to this view is not a believer or infidel (Kafir), but he is in an intermediate position; he did not summon the good qualities and does not deserve the name of praise, then he is not called a believer. Also, he is not an unbeliever because the testimony of faith (Shahadah) and the whole acts of charity are existing in him. He is therefore similar to the believer in the creed, but not similar to him in the work, and he is similar to the infidel in the work, but not similar to him in the creed.

96. Sabra, OP. Cit, pp. 9-10, also Ahmad Mahmoud Subhi, On the Science of Kalam, Vol. 2, Ash'arites, OP. Cit., p.290.

97. Ahmad Mahmoud Subhi, OP. Cit., pp. $25-26$.

98. Ibid, pp. $26-27$.

99. Abu Hamid al-Ghazali, The Chosen in the Science of the Origins of Jurisprudence (al-Mustasfa), OP. Cit., p. 4.

100. Abd al-Karim Zidan, Summary of the Fundamentals of Jurisprudence (Al-Wajiz fi ilm usul al-Fiqh), OP. Cit, pp. $8-9$.

101. Abu Hamid al-Ghazali, OP. Cit., p. 5.

102. Abd al-Karim Zidan, OP. Cit, p. 9.

103. Ibid, pp. $9-10$.

104. Ibid, pp. $15-16$. 
105. Al-Zahiriyah is a jurisprudential doctrine based on that the source of legislation is the appearances of texts from the Quran and the Sunnah only, i.e., there are no opinion or mental deduction in any one of the religion's judgments. The first one of this sect was Abi Dawud alZahiri (d. 882). Ibn Hazm al-Zahiri (994-1069) was also one of its distinguished personalities.

106. Fatehia Al-Nabrawi \& Muhammad Nasr Mihana, The Development of Political Thought in Islam: a Comparative Study (Tatwur al-Fikr al-Siyasi fi al-Islam: Dirassa Muqarinah), Vol. 1, The House of Knowledge (Dar al-Ma'arif), Cairo, 1982, p. 209.

107. Abu Hayyan al-Tawhidi, Enjoyment and Conviviality (AlImta wa al-Mu'anasa), ed. by Ahmad Amin and Ahmad Zein, The General Egyptian Book Organization (AlHay'ah al-Misriyah al-Ama li al-Kitab), Cairo, p. 82.

108. Ibid, pp. $83-84$.

109. Sabra,A.I, Avicenna on the Subject Matter of Logic, The Journal of philosophy, Inc., 1980, p. 748.

110. Frege, Gottlob, On Sense and Meaning, In Peter Geach \& Max Black (ed.), Translations from the Philosophical Writings of Gottlob Frege, Barnes\& Noble books, Totowa, N.J., Reprinted 1988, pp. $56-80$.

111. See Muhammad Ahmad Abdel Qader, Divine Science and its Effects on Thought and Reality (Al-Ilm al-Ilahi wa Atharuh fi al-Fikr wa al-Waq'I), The House of University Knowledge (Dar al-Ma'rifa al-Jami'iyah), Alexandria, Egypt, 1996, pp. $104-119$.

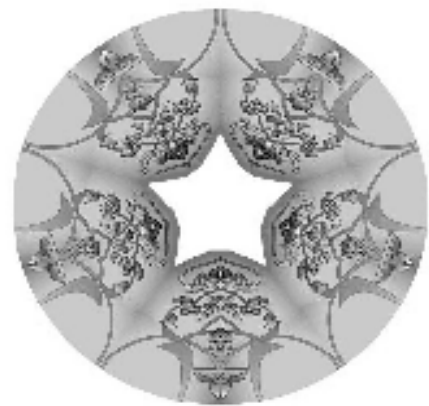




\section{Chapter 3}

\section{Neutrosophic Models of the Arab-Islamic Thought ${ }^{1}$}

\subsection{A Neutrosophic Reading of the Islamic Qiyas}

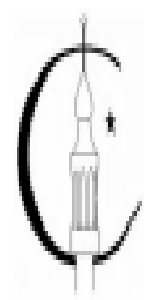

Two-valued logic, be it Aristotelian or classical symbolist, handles a given type of propositions that may be characterized as idealistic. First, it presupposes the idealism of the language used to formulate such propositions, be it natural or artificial. This is in reference to the competence of such a language in expressing all meanings with clarity and accuracy. Second, it presupposes the idealism of man's cognitive abilities as regards his ability to decide for sure what is true and what is false in the world of concrete reality.

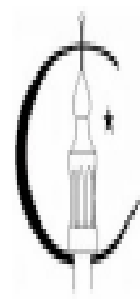
This entails the idealism of syllogism tools (devices) and instruments of experimental detection. In other words, the two-valued logic handles what should be not what really exists. It considers the three Aristotelian laws of thought the deep-rooted and basic rules of human reason. However, there is a great difference between the obligations of such laws and the linguistic or experimental reality as revealed in our life. We are living in an elusive linguistic world, which

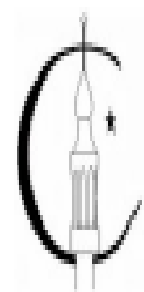
is shrouded in vagueness and suffers from overall deficiency. Meanings of the same word and the same proposition vary in the vast semantic field of such a world. As a result, values of truth oscillate between the two extremes of truth: perfect truth and utter falsehood.

This comes in combination with the discrepancies, which are replete in language and life, as well as our logical and 
mathematical systems. Such discrepancies (Or paradoxes) may cause both truth and falsehood in some propositions. In other words, such propositions may be described as true and false at one and the some time. ${ }^{2}$

The Western world realized such a difference (between rules and reality) more than a century ago. It employed nonAristotelian logics of multiple values to narrow that gap between rules and reality. However, the Arab-Islamic reason had been aware of this for several centuries. It had an undeniable role in handling it in a way that realizes both the purposes and wisdom of Islamic law (Sharia). Muslim thinkers, especially Jurists had realized that the single logical judgment, apart from the explicit judgments of Quran and Sunnah (the Prophet's tradition), may oscillate between the two values of truth and falsehood, covering all values in between. This oscillation is conditioned by time, place, cause and linguistic significance, as well as other circumstances of the event under discussion. Consequently, that logical judgment is merely an 'opinion' (Ra'y) or 'endeavor' (Ijthad) provided by a finite mind. Imam Shafi'i $(767-820)$ expressed this in his saying, «My view is correct, but may prove incorrect, and the view of others is incorrect, but may prove correct».

With this vision of the deductive reason, Islamic jurisprudence (fiqh) realized the significance of multiple explanations. It facilitates the adoption of their religion and rituals at every place and time, as the Muslim has more than a legitimate alternative to choose the fittest freely. So, what is that inference used by Muslims to provide for their judgments? Is it close to the many-valued logic, in general, and neutrosophic logic, in particular?

Jurisprudential inference in Islam is analogy, which is the inferring of the unknown from the existing known. Jurists, generally call it 'Qiyas' (= measurement). But it should be noted that the significance of qiyas for scholars of fiqh differs from that for Muslim philosophers, who used it to refer to 
Aristotelian syllogism of all types, while scholars of fiqh use it to mean 'analogy' or 'similarity comparison'.

This analogy means that the Jurist forges an analogy between a given event that has no legitimate judgment or unanimity (al-Gha'ib = the absent) and another event for which there is a decisive text in Quran or Sunnah, or a unanimous judgment by Muslim Jurists (al-Shahid $=$ the known; existent.) The common element between both is the cause behind the judgment. In this way, the judgment of the latter may be applied to the former with a cause equal in power to that between the cause and its effect. The following pages will reveal that the essence of that Islamic Jurisprudential analogy is so close to, if not identical with, the spirit of the many-valued logic. There are, also, some common features between Jurist syllogism and neutrosophic logic. The most prominent of these points are as following:

1. Both meet the needs of reality. We admit that vagueness is a major feature in our language and knowledge, whether in science or philosophy, or even everyday life practices. We also admit that there are many variables, according to which the values of the truth of our propositions oscillate among truth, falsehood, and neutrality with its different infinite degrees. Then, we are in need of a type of inference that meets the requirements of that reality and fits its nature. This is the neutrosophic logic, the starting point of which was the contradictions of reality and the inaccuracy of our knowledge of it. ${ }^{4}$ This is also the Islamic qiyas, which was closely related to dealings in religious and secular (worldly) issues at its beginning.

2. Also, both neutrosophic logic and Islamic Jurisprudential qiyas handle the neutral and the possible. They do not deny the possibility that truth and falsehood may get merged (meet) at a given spatio-temporal point. Neutrosophic logic has its mathematical, non-Aristotelian, decisive nature, as it is an extension of the classical symbolic logic. But Muslim Jurists and logicians, too, went beyond the limits of the 
Aristotelian logic that has freed itself from the elusiveness of language and reality. This applies especially to the two laws of 'non-contradiction' and the 'excluded middle'. They were not required, at that early stage of their mental and logic development, to have mathematical and symbolic formulation for their propositions, as modern Westerners have done.

3. Both neutrosophic logic and Islamic Jurisprudential qiyas permit going beyond the extreme of truth to ultra-full truth (Truth and beyond). This means that the maximum is not limited to the number 1 . It may extend to the monad $u\left(3^{+}\right)$. This applies to the value of falsehood as the minimum (m) can go under u (zero), not only zero. We have explained this with regard to the neutrosohpic logic. As for Islamic jurisprudential qiyas, the not uttered (the absent) may be more worthy of the judgment than the uttered (the known) and vice versa. For example, if the judge accepts the testimony of two persons, ${ }^{5}$ then the testimony of three persons has more priority. The three are two and more. If atonement is obligatory in manslaughter, it is more obligatory in murder. ${ }^{6}$ If the testimony of the sinner is not accepted, then the testimony of the unbeliever deserves more disapproval, ${ }^{7} \ldots$ and so on. ${ }^{8}$

Let us have a more detailed discussion.

\section{A. Meaning of Fundamental Qiyas}

Qiyas in language means assessing something in terms of another. We measure or assess the earth using the meter. This measurement is used also to refer to comparing something to something else so as to know the proportion of each compared to the other. Later on, the term 'qiyas' began to be used in reference to equalizing two things, be they material or immaterial. An example of material or tangible (concrete) measurement is assessing a given piece of paper with another. An example of immaterial (moral - abstract) thing is saying 
that, «his knowledge is not comparable to hers», that is, their knowledge is not equal. ${ }^{9}$

For fundamentalists (al-usuliyyun), 'qiyas' means annexing what does not have a judgment based on a text to what has as both share the same cause. In other words, it is annexing the branch to the origin for their common features. ${ }^{10}$ Some Jurists, like Imam Shafi'i, opine that 'qiyas' is 'ijtihad', both are one. ${ }^{11}$ Others, like Imam Ghazali $(1058$ - 1111), refuse this opinion, as ijtihad is broader than 'qiyas'. We may practice ijtihad in general issues or shades of meanings and all other methods of proving other than 'qiyas'. Besides, ijtihad, for scholars, means that the thinker (Mujtahid) does his utmost to reach the judgment. Exerting great effort whoever exerts a very little effort is not a fractional of ijtihad. This does not refer to 'qiyas', but to exerting effort. ${ }^{12}$ In fact, the latter view is closer to truth, which may be clear when we consider what is possible and what is not possible to be subject to ijtihad. Take first what does not accept ijtihad:

1. Things that are necessarily crucial in religion such as the necessity of prayer, fasting Ramadam month, paying alms, banning adultery and the like. Such things are so common and known to the ignorant and the scholar equally; and none is excused for being ignorant of them.

2. Judgments that are based on a decisive text, such as the necessity of flogging the adulteress and adulterer as Allah says:

(The woman and the man stained by adultery - in conduct - must be flogged. You flog them with repeated blows one hundred times and do not be moved with compassion on them, expressed in mercy, or else you will have failed to fulfill the duty ordained by Allah if indeed you believe in Allah and acknowledge His system of faith and worship and confess the Hereafter. And it is mandatory that their punishment be witnessed by an assemblage of those in whose hearts reigns piety

$($ Al-Nur $=$ Physical and Spiritual, verse 2. $)$ 
This judgment is based on cogent proof. ${ }^{13}$

Ijtihad may be practiced in one of the following four cases: ${ }^{14}$

1. Judgments that are based on a cogent text, but the significance of this text is liable to interpretation. In this case, Ijtihad means revealing the intended meaning. This is achieved by defining the power of a given word over a given meaning and supporting a given significance at the cost of another. In modern terms, it is a semantic search in the significance of words and their power, be they general or specific, free or bound, etc. Jurists disagree over such matters, but agree on the general rulings that govern the significance of words. An example of such judgments is Allah's saying:

\section{(Divorced women shall remain for three months, or menstrual cycles, in order to regard their condition}

(Al-Baqarah $=$ The Heifer, verse 228)

In this verse, the word 'cycles' is unquestionable, but its interpretation is liable to postulation. It may refer to the 'menstruation', or to the 'purity'. Hanafites accepted the first meaning and contended that the divorced woman may marry again only after the passage of three periods. Shafi'ites claimed that the divorced woman may re-marry only when she is pure from her period three times.

2. Judgments based on a questionable text but cogent significance. These are found in Sunnah (the Prophet's accounts). The scholar, in this case, investigates the conditions of text's truth: its reference, its power and the trustworthiness of its narrators, as well as similar issues of investigations. Scholars of ijtihad widely disagree over issues of such a type. A given Hadith may be unquestionable for a scholar and questionable for another, who does not apply it.

3. Judgments based on questionable text and significance. These are found also in Sunnah. An example is the Prophet's saying: «No prayer without Surat al-Fatiha (the 
introductory surah of Quran)».This Hadith is questionable because the subsequent narrators of Hadith did not report it. It is also questionable concerning the significance because its negation may be the negation of the perfection of prayer, as Hanafites argue. It may a negation of the prayer as a whole, as Shaffi'ites claim.

4. Judgments that are not confirmed by a text nor unanimity. In this case, qiyas, al-Maslaha al-Mursalah (the free benefit), al-Urf (custom), al-Istisihab (continuation), or other means of Islamic proving may be exploited.

Qiyas, then, is a part of ijtihad. But is the more prominent and richer part in regard to its importance, its original and multiple disciplines, the creativeness of scholars in it. This is why the Imam of the two Shrines [Imam al-Haramayn Ibn alJuwayni] (1028 - 1185) says, "Qiyas is the crux of ijtihad and basis of opinion. Fiqh and methods of Sharia stem from it $\gg .{ }^{15}$

\section{B. Cornerstones of Qiyas and its Conditions}

The fiqh-based definition of qiyas reveals that it has four cornerstones as follows:

1. Al-Asl (the origin); it is the case in which a legitimate judgment was certain. It is called the tenor.

2. Al-Fara' (the branch); which is the case for which there is no text. Its judgment is lacking. It is called the vehicle.

3. Hukm al-Asl (the judgment of origin); it is the unquestionable judgment of Sharia, based on the Quran, Sunnah or unanimity. It is transferred to the branch.

4. Al-Illah (the cause); it is the description in the origin, for which a judgment was issued. It is equalized with the origin since it is found in the branch.

The judgment for the branch, which is based on qiyas, is not considered a cornerstone. This is because the judgment of the branch is not a part of the identity of qiyas. It is the fruit of qiyas, or its yield. The scholar of ijtihad has it later than the 
judgment of the origin. This means that it emerges only after the process of qiyas. The cornerstone can not came second to identity.

Let us take an example of these four cornerstones as in the Prophet's saying «The murderer does not inherit». This august Hadith is an origin, which has its legitimate judgment, namely, preventing the murderer from inheriting. The cause of the judgment is murdering so as to inherit, sooner than expected. The guilty perpetrator is punished for his ill-intention by preventing him from inheritance. But there is no text for the judgment of murdering the writer of a will at the hands of the person for whom the will was written. This is a branch in which Ijtihad is used to have a legitimate judgment. The cause of the origin is found in the branch. It is the desire to have something prematurely through criminal means. The judgment of the origin is applied to the branch and the will is not given to its receiver. ${ }^{16}$

However, qiyas is not valid unless four conditions are met. Some of these conditions are related to the scholar and other to the cornerstones. Imam Shafi'i summed them as follows: ${ }^{17}$

1. No one has right to practice qiyas unless he has its tools, which include, for example: knowledge of the judgments of Quran: its obligations, its literature, its verses that may be abolished with the revelation of other verses, its general rulings, its specific rulings, and its teachings. He should also know what may be interpreted ${ }^{18}$ starting with the Sunnah of the Prophet, if not, he moves to the unanimity of Muslims, if not, he moves to qiyas.

2. No one has the right to practice qiyas unless he has full knowledge of previous tradition, accounts and sayings of ancestors, as well as the unanimity of people. Add to this knowledge of differences among people and knowledge of Arabic.

3. Only the some may practice qiyas so that he may be able to distinguish between similar matters and gives opinion only after deliberation and certainty. 
4. The person who practices qiyas should listen to those who disagree with him. He may get attention of something he has forgotten. This will make him move assured of his view. So, he should do his utmost and stick to fairness so as to know why he accepted this and refused that.

As for the conditions of the cornerstones of qiyas, they are varied and multiple. This reflects the desire of scholars of fiqh to stick to accuracy in any legitimate judgment. Such conditions may be summed up as follows:

\section{a. Conditions of the Origin}

Most scholars of fiqh confuse conditions of origin with those of judgment of origin, though both are two distinct cornerstones of qiyas.

This confusion may be a result of their being so close. Imam Ghazali provides eight conditions. Imam Muhammad Ibn Ali al-Shawkani (1760 - 1834) added four more conditions. ${ }^{19}$ However, if we consider the origin as the case of agreement that bears legitimate judgment and the branch as the case of disagreement which lacks that judgment, we discover that the most important condition of the origin itself is not to be a branch of another origin. Its judgment must be ascertained through a text or unanimity. If the origin does not necessarily imply a judgment based on text or unanimity, it does not fit to prove its accompanying meaning. This leads to roundabout wordiness, which is undesirable in logic, while assessing similarity. It may also, in terms of logic, lead to sorites paradoxes, which is a sort of induction that starts with a completely true statement and concludes with a completely false result. This is based on the catenation of forms of proving modus ponons. The explanation of this is that origin which was a branch leads us to compare the third origin to a fourth and the fourth to a fifth and so on. In this case, we end with a result of the origin not similar to the first. Imam alGhazali expressed this in his saying «It is as if you get a store 
and ask for what is similar to it. Then you ask for what is similar to this second store, then the third (to number ten, for instance). You will end with a store that is not similar to the first as five differences get accumulated to reveal the paradox (or discrepancy)». ${ }^{20}$ It is not acceptable, then, is qiyas to refer the origin to another origin that includes it. This may be called the condition of 'independence', in terms of modern logic.

\section{b. Conditions of the Judgment of Origin}

They may be summed up as follows: ${ }^{21}$

1. It should be a practical legitimate judgment based on a text from Quran or Sunnah. Jurists of Fiqh disagreed over the judgment based on unanimity. Some content that qiyas is not valid in such a case - because qiyas is based on knowing the cause of the judgment. If it is found in the branch, it becomes equal to the origin. This does not apply in matters that were proved by unanimity, becomes in such a case of unanimity, it is not stipulated to mention the reference. Without this mention of the reference, the cause of the judgment is not known, and qiyas becomes unattainable. Others argued that the judgment may be passed to the branch via qiyas, even in the case of unanimity, as there are various means of knowing the cause of judgment. These means include alMunasabah [= suitability (or matching)] between the origin and its judgment. In this case, there is no harm due to not mentioning the reference of unanimity. This does not prevent the knowing of the cause. This is the more probable view. If the judgment is ascertained by qiyas only, it can not be made an origin and using it for judgment. This qiyas should be made using the origin of the certain judgment directly.

2. It should be sensible, that is, based on a cause that the mind can realize. Qiyas is based on knowing the cause of the judgment, and its existence in the branch so that the judgment of the origin may be passed to it as both share 
the cause. This is why Jurists of Fiqh argue that qiyas is banned in worshipping judgments. These are the rulings, which Allah concealed their causes upon which judgments were based. No one is ever to know them. This includes the number of kneelings in prayers, the one-hundred floggings of the adulteress and adulterer, the ritual circumambulation around the 'Kaaba' (The Sacred House) in Hajj (pilgrimage) with a definite number of rounds, and the like.

If the judgment of the origin is based on a cause that the mind can comprehend, qiyas becomes valid, if the cause is known, and its realization in the origin is also known. This applies to judgments of utter ban (which were issued on a radical basis, like forbidding the drinking of wine and the murderer's receiving the inheritance of the murdered). It also applies to judgments of license (which were issued with exceptions, as in the forbidding of eating the flesh of a dead beast and similar forbidden foods only out of necessity). ${ }^{22}$

3. It may have a cause that can be realized in the branch. If the cause is confined to the judgment of the origin, and can not be realized otherwise, qiyas is banned. For example, prayers may be shortened in case of travel and fasting may be broken also. The cause of the judgment in both cases is traveling, and the purpose of the judgment is to avoid travail. But this cause - travel - is not realized in a non-traveller. So it can not be used in a qiyas for those who carry out hard work or toilsome tasks.

4. The judgment of the origin should not be confined to it as this prevents its passage to the branch. An example of this is confining the right to marry more than four women only to the messenger, and banning the marring of his wives after his death. This judgment can not be used for qiyas in cases of forbidding or permitting. Another example is confining the acceptance of one witness only to the prophet's Companion Khuzaymah 
Ibn Thabet only. It is a judgment confined to him only based on the Prophet's saying, «whoever has the testimony of Khuzaymah, it is sufficient for him ». It is not valid to equal him with other people whatever his virtue and piety one. ${ }^{23}$

All these conditions aim at purifying the judgment of the origin from any thing that may binder its legitimacy, clarity and a adequacy to derive the judgment of the branch.

\section{c. Conditions of the Branch}

The origin is the subject of the legitimate judgment, which is based on a text or unanimity. The branch is the subject of the desirable judgment through qiyas. Thus, there should be conditions that guarantee its legitimacy and logical accuracy. These conditions include: ${ }^{24}$

1. The cause of the origin should be found in the branch. This is because the condition of passing the judgment of the branch is passing the cause. If the branch is not equal to the origin as regards the cause, the equalization in the judgment can not be validated. It is not stipulated to as certain the existence of the cause in the branch, only suspecting its existence is sufficient, as will be explained.

2. The branch should not precede the origin in being as certained. For example, ablution can not be assessed in regard of 'al-Taimam' (= ablution or getting pure for prayers using sand or clean dust instead of water) in regard to intention.

This is because ablution has priority and precedence to taimam.

3. The cause in the branch should be identical to that in the origin. This means that it should not be lesser than it for the judgment to pass. To be greater is not forbidden as the judgment in the branch may be prior to the judgment in the origin. 
4. The branch should not have a judgment based on a text as the existence of a text negates the need for qiyas. Accordingly, it is said that freeing a non-believing slave does not atone for a vow. This is assessed in a qiyas of the atonement of manslaughter mentioned by Allah in Surat Al-Nisa (= The Women), verse 92. He says:

Observe particularly: It is not right for a believer who has conformed to Islam to kill another believer of the same law unless it be by mistake. And he who kills a Muslim by chance and guilt does not reside in the intention shall run into some consequences in expiation of his wrong. Thus: a) The release of a Muslim slave from bondage and the restoration of his freedom and personal rights, in addition to the payment of blood money to his next of kin as a compensation for the loss of a relative, unless they remit it of their own accord in benevolence. b) If the slain be a believer who has conformed to Islam but his people are an enemy to faith, then you incur only the release of a Muslim slave from bondage and the restoration of his freedom and personal, rights. c) And if he is a Muslim whose people stand friendly with you and their is a binding agreement in which you both enter, then you incur the payment of the blood - money and the release of a Muslim slave from bondage and the restoration of his freedom and personal rights. d) And he who does not have the means to fulfill the divine law as precisely indicated shall have to fast- dawn to sunset- for two consecutive months in expiation of sin; Allah is ever 'Alimun, and Hakimun.

This qiyas is not valid because it violates the text of atoning for an oath. It is mentioned by Allah in Surat AlMaidah (= Heaven's Sent Festive Table), verse 89. He says:

Allah excuses you for oaths in which the guilt does not reside in the intention, but He holds you responsible for the oaths in which guilt resides in the intention, an affront nothing can expiate but : a) Supplying ten 
indigent persons with food equal to the usual standard for your families. Or b) Clothing them with clothes of the same standard. Or, else، c) Freeing a slave from bondage. And he who does not find the means shall fast three days. This is the atonement you make for your oaths if you have carelessly used the name of Allah. Be bound to your oaths. Thus, does Allah expound to you people His revelations that you may hopefully impel yourselves to gratitude

The 'Slave' in this verse is not limited to believers only; it is general. So, it should not be confined to belief in comparison to the expiation for manslaughter.

\section{d.Conditions of the Cause}

The cause (illah) in Arabic language refers to what changes the judgments. It is also a disease that is chronic. ${ }^{25}$ This is why it is used as a cornerstone in qiyas because its impact on the judgment is similar to its impact on the patient. As for its importance, it is the basis of qiyas and its foundation. Qiyas is done and its fruit is yielded only by knowing that cause and verifying its existence in the branch. The scholar of ijtihad realizes that the judgment based on a text is not confined to it, it is a judgment for all events that has the cause of the judgment. This is why the disciplines of Muslims have varied in regard to the logic of the cause, they investigated its conditions, courses and all aspects in complete detail. They used to have authentic intellect that comes close to the modern disciplines of logic, as well as contemporary ones, but, as we said, not in the mathematical form that is more familiar now.

Before discussing the conditions of the cause, and its aspects, we should distinguish between 'cause of the judgment' and 'its wisdom'. The wisdom (al-Hikmah) is the benefit, which the legislator wanted to realize through his legislation. It may crystal clear as in Allah's saying:

Do recite O Muhammad all that has been revealed to you of the Book - the Quran - and establish worship and 
adore Allah, your Creator, with appropriate acts and rites, for sincere prayer checks abomination and immorality. To apply remembrance to Allah is more eminent in point of attainments and achievement, and Allah knows all that you do

\section{$($ Al-Ankabut $=$ The Spider, verse 45)}

And the Prophet's saying: "O young people, whoever can marry, he should do so as marriage is the means of chastity». The benefit may be unclear and unverifiable, as in worshipping rituals, like number of kneelings in prayer or kissing the Black Stone (al-Hajar al-Aswad) during pilgrimage or lesser pilgrimage (Omrah). But 'cause' is the manifest and definite description upon which the judgment was based and related to it, in its existence and disappearance. This is because this description aims at realizing the intended benefit expected from legislating a given judgment. This is why fundamentalists contend that, «Judgments are related to their causes not their wisdoms». This means that the judgment exists once its cause exists, though its wisdom may be hidden in some cases. The judgment gets invalid once its cause vanishes, though its wisdom may exist in some causes. For instance, the cause of forbidding wine is getting drunk; the wisdom of this is to preserve minds of people. So, once the cause exists, forbidding exists. This link between the judgment and its cause is that the wisdom, which may be unclear, may also be indefinite. People may disagree about it. In this case, no judgment may be based on it as this will lead to confusion and chaos in judgments. This, in its turn, will disturb the command of carrying out the judgment, and allegations and claims of being unbound to carry out the judgment will vary. For example, the wisdom of permitting breakfasting in Ramadan is avoiding toil and hardship. This is an evaluative and indefinite matter. So, the legislator conditioned this judgment with a definite thing: traveling or sickness. Both may be the subject of the wisdom of the judgment. ${ }^{26}$ Also, linking judgments with their causes entails the inferring of 
legislations and venerating them. They are not to be a subject of manipulation so as to drop some commands once the wisdom is realized (al-Ghayah = the goal). An example of this is an ignorant person's saying: «If the wisdom of prayers is to prevent abomination and evil deeds, it is possible, then, to drop prayers, along with the abandonment of abomination and evil deeds $\gg .{ }^{27}$

The conditions of the cause vary in number from a Jurist to another. Al-Shawkani, in his Irshad al-Fuhul (= Guidance of the Masters) enumerates twenty-four conditions, the most important of which are: ${ }^{28}$

1. It should be crystal clear and verifiable as existent or nonexistent in both the origin and the branch. If the cause is a hidden description, the legislator defines a clear thing instead, which indicates it. This applies to murder which the cause of retaliation. But the 'intention' of killing is a moral issue known only to the perpetrator. This is why the legislator has defined a manifest thing for it to indicate it. This is the tool of murdering, such as a sword, a pistol, a rifle.

2. The cause should be definite. This means it should have a specific reality (or truth) that does not get changed with changes in persons or circumstances. This applies to differences of hardship or toil with different persons or ages of these persons. Thus, hardship in work is not a cause for shortening prayers in case of travel.

3. It should be a suitable description of the judgment. In other words, the legislation of the judgment should entail the existence of a given benefit or obliteration of a given evil. For example, theft is a suitable description for the legislation of truncating the hand of the thief. Linking this truncating of the hand with theft entails the preservation of people's money and property. Accordingly, descriptions should not be used to justify things that are not in match with the judgment. These are known as arbitrary descriptions, such as the color of wine, its liquidity, its 
taste. None of these may be a suitable description for forbidding wine. The thief's being rich, a man of authority, or a nomad or the person who was robbed being poor or a worker one not suitable descriptions for the judgment of truncating the hand of the thief.

4. The cause should not be confined to the origin. It should go beyond it to include similar or analogous things. The cause of shortening prayers is traveling not hardship. So, any other hard work should not be compared to this. In contrast, drunkenness is the cause of forbidding wine. This description is found in every sort of drunkenness - causing drink, then, it is, not confined to the origin.

5. The cause should be influential in the judgment, which has existed due to it only. Intentional murder is the cause of retaliation and drinking wine is the cause of being drunk, for instance.

6. The cause should be cogent; it is not refutable by a text or unanimity. No legitimate proof is there to abolish it. The scholar of Ijtihad may, at first sight, imagine that a give description is suitable as a cause for a given judgment, while, in reality, this description contradicts a text and legitimate evidence. In this case, this description has no value nor suitability for judgment. For example, combining the male and female may be taken as a suitable description (cause) for giving both equal portions of inheritance. This is definitely wrong. The legislator has ignored the matching of this description with the proposed judgment as Allah says:

\section{Allah commends you that the male shall inherit the equivalent of what is apportioned to two females}

$($ Al-Nisa $=$ The Women, verse 11$)$

7. The cause should not be refuted by other stronger causes. This is why jurists suspend the punishment of theft in time of war lest the thief should flee to the enemy and add to his power. 
8. The cause should not entail a judgment for the branch and another for the origin. It should not entail two contradictory judgments either, by requiring two opposites.

9. The cause should be related to the judgment constantly. If it appears, in any form, the judgment should accompany it.

10. The cause should also be negatively related to the judgment. Once it vanishes, the judgment disappears. But this does not imply that one thing may not have two caused. For example, forbidding marriage is not caused by kinship only, but by breastfeeding and related marriages. So, non-existence of kinship is not enough to permit marriage. ${ }^{29}$

It may be noticeable now that the conditions, outlined by fundamentalists, for the cause excel those outlined by modern philosophers for the relationship between the cause and the effect, in quantity and quality. This includes the 'method of eliminations' of the English philosopher Francis Bacon (1561 - 1626) and the inductive methods outlined by the English philosopher John Stuart Mill (1806 - 1873) for the scientific hypothesis: 'the method of agreement', the 'method of difference' the 'method of combining agreement and difference', the 'method of concomitant variations' and the 'method of residues'. The method of agreement, for instance, is analogous with the condition necessitating that the cause be progressive. This applies to the methods of difference and elimination, which are similar to the condition that the cause be retrogressive, etc. ${ }^{30}$

The conditions of the cause, as outlined by fundamentalists, in fact, include the contributions of later western philosophers in developing the deductive method. Besides, they include the conditions outlined painstakingly by contemporary logicians for deductive patterns, especially those by the German mathematician and logician M. Pasch (1843-1930), founder of the modern Axiomatic movement. ${ }^{31}$ The condition that the 
cause should not include two opposites is identical with the condition of 'consistency' or 'non-contradiction' in the deductive system. Also, the condition that the cause be influential and not limited to the origin is identical with the condition of 'completeness' or 'saturation', etc. ${ }^{32}$

\section{Paths of the Cause}

These are the means, by which we know the cause in the origin. It is the evidence of the existence of the cause. Some jurists enumerated them as ten, but they may be classified into three sections: the text, unanimity and deduction. The last second may be subdivided into some paths of Ijtihad governed by mental inference. Let us discuss the most prominent in detail.

- First Path: Al-Nas (= the Text):

This means the Quran and Sunnah. The text may be overt in its indication to the cause. In this case, evidence is divided into decisive and speculative. Evidence may be also by gesture or indication only.

1. The text's reference to the cause decisively: This is realized by using linguistic forms and words that refer to causality. Examples include 'therefore', 'thus', 'this is why', 'the reason of this is' and the like. An example of this is Allah's saying:

Therefore, all that Allah has bestowed on His Messenger of the resources gained from the villages' inhabitants under such circumstances belongs to Allah and to the Messenger, to the kindred and the orphans, and to the indigent and the wayfarer who, due to lack of means, travels on foot. This is to prevent such gains from passing into the hands of the wealthy among you. And whatever the Messenger commands you to do, you must do, and whatever he forbids you to do, you do not do, and entertain the profound reverence dutiful to Allah; He punishes severely $(A l-H a s h r=$ The Thronging, verse 7$)$ after 
mentioning the sources of 'al-Fai' (property taken from the enemy) such as the tribute (Khiraj) and spoils without fighting. These are for the poor and the needy. This text states overtly that the cause is preventing money from being distributed among the rich, not others. The same applies to Allah's saying:

Such Messengers were sent as spectacles and warnings so that people shall have no excuse nor can they hold a plea against Allah in mitigation of censure after they had been warned by Allah's Messengers. And Allah has always been Azizun and Hakimun

$$
(\text { Al-Nisa }=\text { The Women, verse 165) }
$$

The text overtly states that the cause of sending messengers is that, So that people shall have no excuse nor can they hold a plea against Allah. An example from Sunnah is the Prophet's saying: «Asking for permission to enter is made for fear of seeing». This is a decisive text showing that the cause of asking for permission before entering a house is to prevent the visitor from seeing what he is forbidden to see. By analogy, one is forbidden to peep through a window to see what is inside another person's house.

2. The text's reference to the cause indecisively: In this case, the text refers to the cause, but it may have another acceptable interpretation. Hence, the reference is overt and speculative. An example of this is Allah's saying:

A Book - the Quran - the Source of enlightenment - We have revealed it to you O Muhammad to guide people into all truth and to lead them, Allah willing, out of darkness and superstitions of later times and out of want of spiritual and intellectual sight into illumination and enlightenment. As well as to direct them to the path of righteousness, the path of Allah, the AL-Aziz (Almighty) and AL-Hamid (to Whom are extolled the glorious attributes)

$($ Ibrahim $=$ Abraham, verse 1$)$ 
His saying 'to' may be subject to interpretation. It may also refer to the end of whoever believes in the Book and abides by its commands and banning.

3. The text's reference to the cause by gesture: 'Gesture' (al-Ima') in Arabic language means referring to something or calling attention to it by stating one of its aspects denoting it. An example of this is having an emphatic 'verily' in a sentence following a sentence that includes the judgment. An example of this is the Prophet's saying, in answering a question about the purity of the water licked by a cat, «It is not unclean, the cat is among those that wait upon you». He did not say 'because' or 'as', He just indicates that the cause of not being unclean (in reference to the water licked by the cat) is that the cat waits upon men. If this were not the cause, the mentioning of "waiting" would not be useful. ${ }^{34}$

\section{- Second Path: Al-Ijma' (= the Unanimity):}

It is the evidence that the cause influences the judgment. An example of this is the unanimity about the priority of the full sibling - being born of the some father and mother - as the cause of his priority in inheritance. By analogy, he is prior to the stepbrother in regard to self-dependence.

\section{- Third Path: Al-Istinbat (= the Deduction)}

This includes the path of probing and dividing (al-Sabr wa al-Taqsim), matching judgment and cause (al-Munasaba), regularity (al-Tard), inseparability (al- Dawaran) and refining reason (Tanqih al-Manat).

1. Probing and Dividing: 'Al-Sabr' (= probing) in Arabic language means examining something and testing it so as to reach its reality. 'Al-Taqsim' (= dividing) is segmenting something and breaking it down to its components. Its essence is based on the scholar of Ijtihad who counts all descriptions in the origin, which he considers suitable as a cause for the judgment at the first 
moment. Then he investigates and tests it to eliminate what he deems unsuitable and keeps what he deems suitable as a cause. The result is realizing that a given feature, not others, is the cause of the judgment. In this process, the scholar of Ijtihad is guided by the conditions of the cause. He keeps only the definite, manifest, suitable, and exhaustive description (or feature). He should, also, reach the utmost of analysis till he is unable to bring out any description that rivals the description he has kept as a cause. If another scholar is similarly incognizant of any other description, he says, "You have what I have». If that scholar excels him in cognizance of another cause, he should warn him so as to investigate how far it is true or false. ${ }^{35}$ Let us have an example of this path. We have the text of forbidding wine. Some scholars of Ijtihad did not get cognizant of the Prophet's saying «Every drunkenness-causing drink is forbidden». They may have knowledge of this saying, but they did not approve it. So, they investigate the cause of forbidding wine by enumerating the descriptions that may include the cause. For example, the wine is from grapes, it is liquid, it causes drunkenness, etc. Then, they investigate all these descriptions guided by the conditions of the cause. They obliterate the first description, as it is deficient, while the cause should be exhaustive; they abolish the second description (the wine being liquid) because the link between it and the judgment is non-existing. The third description of 'intoxication' is kept as it is a manifest description, which suits the judgment. ${ }^{36}$

This path is similar to the method of philosophical analysis of the contemporary English philosopher C. E. Moor. (1851-1931). His analysis is based on two steps: division and distinction. But the basic criterion is the logical equivalence between analysandum and analysans. Moor's aim of division is to segment the 
concept under investigation into all other concepts that compose it. He hypothesizes that the concept is a compound. By 'distinction', he aims at enumerating all possible uses of the word denoting that concept. He tries to spot the common feature in all these uses. If we exclude all concepts whose meaning is far from the concept under investigation, we distinguish it from others. In this way, we reach a logical equivalence that attains the identity of the meaning or synonymy between analysans and analysandum. ${ }^{37}$ Moore adopted his method on concepts or issues to define their accurate meaning, while fundamentalists adopted the path of probing and dividing on the legitimate judgment to spot its accurate cause. But, Moore suggested that there is one specific meaning for the concept, or that it had various meanings and we should extract their common feature. Meanwhile, fundamentalists did not exclude the fact that the one judgment might have more than one cause, on the one hand. On the other hand, Moore had eventually realized that his method of analysis realized the link of 'implication' not 'equivalence' between the concept and the concepts revealed by analysis. This is exactly what fundamentalists had stressed in their path. The judgment, for them, is a consequence of the cause, not an equivalent to it. They also perfected this path with another, namely refining the reason.

2. Suitability or Al-Munasaba (matching - conveniency). It means that the scholar of Ijtihad investigates the origin of certain judgment to find out a description perceived by the mind as suitable (valid to be linked with the judgment). If the scholar finds out a suitable, distinct description, he considers it the cause. If the description is not suitable, not distinct or stained with features that have no impact on the judgment. The scholar has to do his utmost to distinguish it in accordance with the path of refining reason. If there are no suitable descriptions, the 
scholar has to adopt the path of probing and division. Suitability, however, is not matter of personal taste or inclination. It has definite rules. It does not become approved unless the legislator assigns it a sort of personality. This is why fundamentalists had classified the suitable description into the following types, on the basis of the legislator's perspective towards it as valid or invalid: ${ }^{38}$

a. The Influential Suitable (Al-Munasib al-Mua'thir):

It is the description considered by the legislator to be a cause for the judgment itself. This is the supreme type of the suitable. Proponents of qiyas do not disagree over its validity for qiyas. An example of this is Allah's saying,

They ask you, O Muhammad, for information relative to women's menstruation and whether sexual congress is permissible in its duration. Say to them: It is an unwholesome phase in the woman's menstrual cycle fraught with systemic changes including personality alteration and a general decrease in resistance to disease. Therefore, leave women alone during menstruation and do not approach them until they are clear of it and have cleansed. When once they are ritually conditioned, you may feel free to approach them in the manner Allah has ordained. Allah likes those who in lowliest plight repentant stand and those who free themselves of physical, moral and spiritual defilement $(A l$-Baqarah $=$ The Heifer, verse 222), the judgment requiring that men do not have sex with their wives while they have a period of menstruation is certain due to this text. It is explicit in its wording and stating that the harm due to menstruation is the cause of the judgment. So, it is an influential description (the harm). 
b. The Proper Suitable (Al-Munasib al-Mula'im): It is the description for which the legislator does not provide an evidence as the very cause of the judgment. However, there is legitimate evidence, based on a text or unanimity, that it is proper the cause of the genus of the judgment. Its genus may be considered the cause of the judgment proper. Its genus may be considered the cause of the genus of the judgment. Let us have one example only to illustrate the description regarded by the legislator as the cause of the genus of judgment. This is the certain right of the father to marry his young virgin daughter. The cause of this judgment, according to Hanafites, is young age, not virginity. Hanafites found their viewpoint on the fact that the legislator has regarded this description as salient (vital-important, worthy, effective) when it was made the cause of being a trustee on money. Trusteeship on money and that on marriage are of the some genus. Thus, the legislator considered young age a cause for anything under the genus of that trusteeship.

c. The Free Suitable (Al-Munasib al-Mursal): It is the description, for which there is no special evidence of validity or invalidity. However, providing a judgment based on it achieves a benefit verified by generalities of Sharia as a whole. Examples of this include gathering the Quran, coining money, building prisons, etc.

3. Regularity, Retrogression, and Inseparability (AlTard wa al-Aks wa al- Dawaran): 'Regularity' (al-Tard) means that the cause is related to the judgment in case of existence not non-existence. In other words, the cause is not found unless the judgment is there. 'Retrogression' (al-Aks) is the abolition of the judgment with the disappearance of the cause. 'Inseparability'(al-Dawaran) means that the judgment is related to the cause in their 
existence and disappearance. Fundamentalists had realized that regularity only was not adequate as two phenomena may co-occur without having a higher relationship between them. An example of this is the collocation (bond, co-occurrence) of day and night or the essence and the symptom. The cause may be abolished while the judgment does not get abolished if it is the effect of more than one cause. This was why fundamentalists supported the existence of both regularity and retrogression together, or both inseparability and running. Grape's juice is not forbidden at all before it becomes intoxicating. Once it becomes intoxicating, it is unanimously forbidden. One this intoxication is gone, it is not forbidden any more. When forbidding rotated with intoxicating in their existence and non-existence, it is proved that the grape's juice is not forbidden for its being made from grape, for its being a liquid nor for its color. It is forbidden for its intoxicating effect. Accordingly, they used this for analogy and issued a judgment forbidding all intoxicating drinks even if they are not called wine. ${ }^{39}$

4. Refining Reason (Tanqih al-Manat): 'Refining' (Tanqih) in Arabic language means 'chiselling', 'improving' and 'making distinct'. 'Refined speech' has no padding (wordiness). Reason refers to the cause. It is a place name originally (the hanging place). If you hang something, you relate it. This was applied to the cause as the legislator related the judgment to it. ${ }^{40}$

Fundamentalists use this 'refining' to mean trimming the cause by removing descriptions unsuitable for cause giving. This is why some jurists regard refining reason and probing one thing, though the former is more exhaustive.

An example of this is found in Sunnah. A Bedouin came to the Prophet while in panic to tell him that he (the Bedouin) has had sex with his wife deliberately on a 
Ramadan day. The prophet imposed an expiation on him. So, this expiation is a legitimate judgment on the Bedouin and whoever does a similar act. But the text includes some descriptions and does not specify one as the cause of the judgment. For example, the man who had sex was a Bedouin, his wife was his sex partner, the incident took place in Al-Madina al-Munawwarah (a sacred city in Saudi Arabia), the intercourse took place in a specific Ramadan, and the intercourse was deliberate. For the scholar to reach the cause to which the judgment was related, he has to chisel (trim, refine, revise) such descriptions to purify them by removing all what can not be the cause. He will realize that the intercourse has occurred deliberately on a Ramadan day. This is the cause of the judgment of the expiation.

'Extracting Reason' (Takhrij al-Manat) and 'Editing Reason' (Tahqiq al-Manat) are fundamentalists terms that get confused with this path. The former refers to getting out the cause of the judgment not proved by a text nor by unanimity. This is achieved by following one of the paths of cause. 'Editing reason' means investigating and examining the actualization of the cause, as proved by a text, unanimity or deduction, in a given event other than the event of the text. If the cause of avoiding sex with women during their period is harm, the scholar investigates how far the cause is actualized in post-natal menstruation. If this harm is proved, the scholar uses qiyas, the judgment of the origin extends to the branch: that is women should be avoided while in their post-natal menstruation. ${ }^{41}$

\section{Types of Fundamental Qiyas}

The pivot of qiyas is the common cause of the judgment between the branch and the origin. Accordingly, fundamentalists had divided qiyas into three types based on 
the power of the cause that justify the judgment in both the branch and the origin. These types include: ${ }^{42}$

1. Qiyas of Priority (Qiyas al-Awla or qiyas from bottom to - top), in which the cause of the judgment in the branch is more powerful and clearer than that in the origin. This makes the degree of truth of the judgment increase in the branch till it exceeds the full number one, in accordance with the Neutrosophic logic, assigned to the judgment of the origin. An example of this is Allah's saying, concerning His recommendation of good treatment for the parents:

Allah, your Creator, has ordained, that you worship no one but Him and you adore Him with appropriate acts and rites and that you commit your parents to your kind care. When one or both of them attain old age they expect your friendly disposition by your conduct to them. Therefore do not offend them by giving vent to an exclamation of impatience or disgust nor should you rebuke or reprimand them and let your dissertation with them be a sweet discourse

$($ Al-Israa $=$ The Night Journey, verse 23)

In this august verse, Allah forbids us to say the slightest word of contempt to parents. From this forbidding of a simple act, Muslim jurists have extracted the prohibition of the more serious act of beating the parents.

2. Qiyas of Equality (Qiyas al-Musawi or qiyas of the same degree), in which the cause of the judgment in the origin is existing in the branch as far as it is achieved in the origin, i.e., in an equal truth - value. An example of this is the Quranic verse:

Indeed those who wrongfully eat the orphans out of their property simply consume but fire into their bellies and shall suffer conflagration

(Al-Nisa $=$ The Women, verse 10$)$

The cause of judgment in this verse is the aggression on the orphan's money. Inasmuch as the burning of the 
orphan's money is equal to the fact mentioned in the text concerning the cause, then the judgment is the same as in the verse.

3. Qiyas of Minimum (Qiyas al-Adna or qiyas from top - to - bottom), in which the achievement of cause in the branch is weaker and less pronounced than in the origin, although the two causes are equal in the verification of meaning, by which the description became a cause. An example of this is the intoxicating; it is the cause for the prohibition of wine, but it may be more applicable to another drink, such as beer, albeit the feature of intoxicating exists in both.

\section{E. Qiyas between Certainty and Probability}

Does Islamic jurisprudential qiyas lead to certainty or probability? Aristotle states that the analogy leads to the probability. However, Islamic jurists insisted on denying the similarity between their qiyas and analogy, because that may lead to the suspicion in most of the legal judgments, which are not based directly to the text of Quran or Hadith. Nevertheless, they did not hasten to the rule that qiyas leads to certainty, because what is resulting from a mental reasoning does not rise up to the revealed judgment. Imam Al-Ghazali states: 'It is should be recognized that the certainty in the theories is one of the rarest things in existence. As for the probability, it is the easiest thing to get. The uncertain judgments in the jurisprudential issues are the likely thing that we use when we hesitate between two matters: boldness or retreat'. ${ }^{43}$

Therefore, fundamentalists argued that the relationship between cause and effect is not the same degree of influence or necessary, but it is divided into five types that make us close to the many-valued logic, which are:

1. A cause leads to the effect certainly. The relationship here means that the degree of similarity between the cause of origin's judgment (Hukm al-Asl) and the cause of branch's judgment (Hukm al-Far'a) up to the full truth (just one). In 
other words, it means the decisive recognition of the need of emanation of the effect from the cause (as exploitation in the usury and the intoxicating in the wine). We previously reported that the degree of truth can be more than the just one, as in qiyas from bottom - to -top (qiyas min al-Adna ila al-A'ala).

2. A cause leads to the effect probably. The probability means that we have two possible things, but one of them is clearer than the other, such as the human's opinion that the thick clouds are carried with rain, although the clouds may clear away without rain. Also the jurists' think in the validity of their legal opinions, although they may permit any thing to be otherwise. ${ }^{44}$

The examples in jurisprudential qiyas are many, including: the hardship, which is the cause of the permissibility of breakfast for the traveler and patient in the daytime of Ramadan month; it is a moral thing, which differs from one to another. As well as the partiality of one to his relative in the testimony, it is possible that the witness does not bias to anyone, whatever the degree of the relationship. In addition, the judge's arbitrariness when he rules in the case of anger, it is possible that he is not influenced by the anger...etc. In each of these cases, we can take the opinion and its opposite.

3. A cause leads the effect suspiciously. The suspicion is the permissibility of two things without any priority of one of them for another. Or it is the recognition of cause with the possibility of the existence of an equal contrary one. For example, the eating of onion and garlic before going to the Mosque (al-Masjid), here the judgment is based on the suspicion that the widespread odor from the mouth will hurt the congregation. However, it is equally likely that it does not lead to the hurt.

4. A cause leads to the effect illusionary. The meaning of illusion here is the recognition of cause with the existence of another one that more likely and powerful, such as the 
judgments of pessimism, prohibited by the Prophet's saying: 'There is no infection or pessimism in Islam, and I like the auspice'.

5. A cause does not lead to the effect at all. Here, the degree of implication's power between the cause and the effect (i.e., the value of judgment) is the complete falsehood (zero) or less, as it reflects either the ignorance of the cause, namely the full unrecognizing of it, or the complex ignorance; i.e., the recognition of a cause that is opposite to the reality according to the Quranic text. An example of this case is the unwillingness to gave in benevolence or spend in divine service because of the fear of poverty. Who says that is so ignorant of Allah's saying:

And whatever you spend in His cause and in benevolence, $\mathrm{He}$ replenishes it, for $\mathrm{He}$ is the most capable of meeting peoples' needs and the best Purveyor of all requisites of His creatures

$\left(S a b a^{\prime}=\right.$ City of Saba', in Yamen, verse 39)

Another example is the Prophet's saying: «There is no day that passes to the servants but two Angels come down from the heaven; one of them says: 'O God, give the compensation to who are charitable in divine service', and the other says: 'O God, give the impairment to who are scrooge'».

There is no doubt that the probabilistic nature of qiyas had allowed for the multiplicity of the jurists' views, and for their disagreement in what is the subject of Ijtihad. This has had a different variety of reasons, for example: ${ }^{45}$

1. Their disagreement in the interpretations of the words contained in Quran and Sunnah, such as their disagreement in the meaning of the word 'Qura' (= Lunar month); does it mean the duration of women's menstruation or their cleanliness?

2. Their disagreement in understanding the distinctions relating to the structure of Arabic language and the 
formation of sentences, as well as their disagreement in the receiving of speech; does it mean the truth or involves a metaphor?

3. The existence of some Quranic verses and Prophet's sayings, which if we combine them to each other, the minds must differ in what we can or cannot deduce from them.

4. The difference of some Prophet's sayings in a specific subject, and the awareness of some jurists of them more than others, or the validity of these sayings according to some jurists, and the invalidity of them according to others.

5. The variation of jurists' ability in the measurement and deduction, or their differences in the linguistic capacity and the awareness of Arab methods and the significance of Arabic speak.

6. The natural and social environment' influence on the jurists. As well as the variation of spatial and temporal variables with clear and necessary influence on the adequacy and the acceptance of the jurisprudential judgment.

However, this disagreement, in fact, was not of the reprehensible type, which may lead to the disunion of nation and scattering the seeds of hostility among people and undermining the foundations of civilization. But it was a reflection of the richness of thought, the flexibility of Islamic law (sharia), and the emancipation of the Islamic mind from rigid dichotomies, in order to enter the world of mental and real possibilities strongly, in all their infinite dimensions. In other words, we can say that the difference of jurists, rather, comes within the framework of cooperation and correspondence, which is indispensable in people's matters of religion and of life. The different jurisprudential judgments do not go beyond the right or the closest to it; and the wrong or the closest to it. The ranks of closeness and remoteness are 
different, but this type of difference does not require the hostility, or the separation of address, or the wastage of unity. Al-Shatibi (d. 790) says: «The difference in some of the jurisprudential issues due to either their rotation between two clear extremes that conflict in the eyes of jurists, or to the absence of some evidences, or to the ignorance of evidence. In fact, the second reason does not reflect any disagreement, because if the jurist had known what was hidden, he would have retracted his judgment»r. ${ }^{46}$

There is no contradiction between the importance of difference and the Prophet's prohibition of the wading into the allegorical revelations, where He said: «the lawful (al-halal) is clear, and the unlawful (al-haram) is clear, and there are comparable matters between them, which are not known by many people. So, who avoided these comparable matters, he will save his religion and honor, and who dropped in these matters, he dropped in the unlawful». What we understand from the text of hadith that the things are three sections; the first is the clear lawful, such as eating of worthy food, marriage, and others. The second section is the clear unlawful, such as wine, usury, adultery, and others. The third one is the section of suspicious things, which are not clear for many people. The significance of these last words is that God has bestowed on the nation with the jurists, who can know the judgments by means of the text or qiyas or any other means. The evidence of that is the Quranic verse:

When -these people- learn by credible or incredible information a subject or report or talk to the credit of the Muslims or against them, they spread it as news and circulate it by way of rumour. Had they referred it to the Messenger and, in his absence, to those in authority among them, it would have been in accordance with divine standard. The Messenger and / or those in authority would have intelligently examined the news and rationalized the matter and would have known the facts and placed them in their true bearings. Had it not been for Allah's 
efficacious grace and mercy abounding in you, you people would have been attracted to AL-Shaytan and espoused the opinions of those with characteristics befitting him, except a few

(Al-Nisa $=$ The Women, verse 83)

If the thing hesitates between the lawful and the unlawful, and there is no Quranic or Prophetic text or unanimity on it, then the jurists must endeavor to clear it. Perhaps we can express this by the following neutrosophic form:

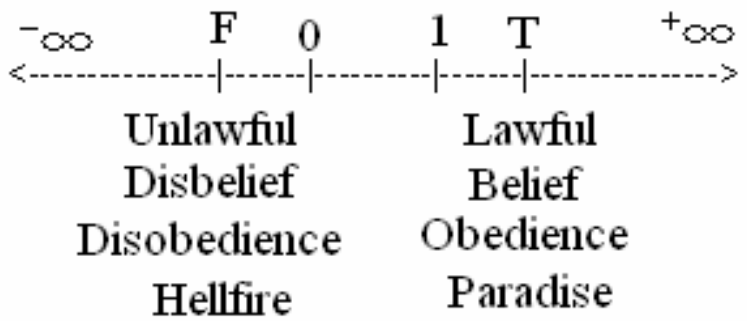

In this figure, the lawful (as well as belief, obedience and Paradise) represents the absolute truth, and the unlawful (with disbelief, disobedience and fire) represents the absolute falsehood. Among them, i.e., between $\langle\mathrm{A}>$ and $<$ anti-A $>$, there are infinite numbers of the convergent cases. As for the increase of the truth-value to the post of number one, and the diminution of the false-value to the pre-zero, it is fixed by many Quranic verses. Some of these verses:

(He shall requite those who vested their deeds with wisdom and piety with more than they expect; more than what is commensurate with their pious deeds. Never shall their faces be overlaid with unbroken and oppressing, mournful and painful gloom nor with humiliation and shame; these are the inmates of Paradise wherein they wit have passed through nature to Eternity

$$
\text { (Jonas }=\text { Yunus, verse 26) }
$$

The infidels must not think that the respite We grant them is for their own good; We simply give them plenty of 
rope to allow them free action in order that they commit themselves further to the evil line of conduct. There awaits them a condign and humiliating punishment

(A'l Imran = The family of Imran, verse 178)

It was He Who inspired peace and tranquility to the hearts of the believers whose breasts are filled with reverential awe, so that their hearts be impressed with the image of religious and spiritual virtues, and their deeds with wisdom and piety. And to Allah belong the forces, specific and generic of the heavens and the earth, and Allah has always been Alimun (Omniscient), and Hakimun (Wise)

$($ Al-Fath $=$ The Opened Gates of Victory, verse 4)

But the Prophet has warned us to the possibility of reversion of the situation of belief upside down, where he said: «One of you engages in the deed of the Paradise's people, till the distance between him and the Paradise becomes only one arm, then he engages in the deed of the Hellfire's people, to enter it. Also, one of you engages in the deed of the Hellfire's people, till the distance between him and the Hellfire becomes only one arm, then he engages in the deed of the Paradise's people, to enter it».

Are not these examples representing a genuine creation that characterized the Islamic mentality many centuries before? Does not the Islamic qiyas reveal an Islamic genius, which we miss now, in the work in the principles of contemporary logic in front of a reality that is characterized by change, while we insist today, in the Middle East, on freezing the mind and importing the ready-mode ideas, with its positives and negatives, from the West?

\section{F. Ijtihad and the Renewal of Religious Thought}

Qiyas was not the only way in which the jurists and scholars moved to deduce the legal judgments, but the developments of life and the complexity of problems in the daily transactions after the expansion of Islamic State have required diversifying 
the ways of deduction. So they tended, for example, to the application of the method of approval (or admiration, Istihsan).

Al-Istihsan is the abandonment of an obvious qiyas for a hidden one, so that if the application of the judgment leads to the forfeiture of a legitimate interest, or causes damage, it will be necessary to abandon the general rule in accordance with the requirements of special interest. There are many examples for that, including: ${ }^{47}$

1. Omar Ibn al-Khatab, may Allah be pleased with him, suspended the application of the punishment of amputation of the hand on a slave, who stole his master because he prevented his fee. The reason of application of the punishment is the extortion of rights, but Omar suspended it for an opposite reason; that is the deprivation of rights, therefore, he threatened the master to apply the punishment on him if he does not give the slave his fee.

2. If anyone makes a vow, he must perform it, as an application of the Quranic verse:

Thereafter they shall be permitted to remove the unwanted biological accumulations on their bodies hair, dirt, nails, and to fulfill their promises and pay their vows -if any- and again go round the ancient Sacrosanct House in procession

(Al-Hajj = The Pilgrimage, verse 29)

Abd Allah Ibn Abbas was asked about who made a vow in a disobedience of God, he said: 'If the vow for Allah, then the vower lies to Allah, and if it for the Satan, then the vow is untrue'.

3. The Messenger did not allow taking fees for the reading and education of Quran, but when the life complicated, the jurists gave a legal opinion that this is permitted, because if the men maintained the performance of these religious duties, they would be unable to acquire their nourishment, 
then hungered and perished. Moreover, if they sought to their livelihood, they would stop to do these duties.

4. Imam Ibn Taymiyyah (1263 - 1328) says: «In a time of the rule of Tatars, I passed with my friends by a people drinking wine. Some of whom with me denied that deed, but I denied that they (my friends) are trying to forbid the evil, and I said: Allah prohibited wine because it alienates from keeping Allah in mind and from the act of worship. However, the wine alienates those people from the murder of soul, the capture of posterity, and the rape of funds. Let them with their wine». Here the rule is the selection of lesser of two evils.

Jurists also resorted to the deduction using the free benefits (= al-Maslih al-Mursalah), namely those which were not specified expressly by a Quranic text or Hadith, although they can bring benefit or repel harm. Jurists have identified three basic conditions for these benefits, which are: ${ }^{48}$

1. If the benefit is not based on a definitive text of the Quran or Hadith, it is not permissible that there is an opposite text of it. Accordingly, the surrender to the enemy in the name of peace is not one of the free benefits, because it is opposite to the principle of struggle (jihad).

2. It must be a genuine benefit, not imagined one; thus, any convention for the exchange of goods with a non-Muslim side, which is stronger than the Muslim side (and has aggressive goals and ambitions in the Muslim State), is an imagined benefit.

3. It must be public benefit, not special; thus, the enactment of a law for the benefit of Governor is not one of the free benefits.

These deductive ways, and others, made the judicial judgments and the religious fatwas (the formal opinions) exactly express the flexibility of Islamic religion, as required by the transactions among people, and as required by the 
spatial-temporal dimension of the event, in an evenhanded middle between the stability of Aristotelian syllogism and the relativity of Sophists.

In addition, the neutral nature of Islamic jurisprudence, especially during the era of the Abbasids, was demonstrated in the division of who are employed in it into two major members, both of them raise the banner of tolerance and appreciation for each other. The first is the school of Hadith (ahl al-Hadith); its emblem is the commitment of text due to its holiness. The second is the school of opinion; its emblem is the interpretation if the text seems as opposite to the mind, or for compliance with the changing circumstances. The first centered in Hijaz, where the Massenger's Hadith has started and completed, and where the listeners of the Messenger are many and it is difficult to counterfeit His sayings by any one. The second school started in Iraq, where the life got complicated and the issues branched, hence, it was necessary to have legislations and opinions issued by the jurists. The people of Hadith were accepting hardly the phrase: «I will endeavor to come to my own opinion», while the people of opinion were accepting hardly the phrase: «The Messenger of Allah said such and such», because they are fearful of the falsification or the weakness of Hadith. Moreover, the people of Hadith hated the question about an event that did not occur, in addition to the assumption, the question: «do you see if such and such...?». They were interested in Hadith, including the weak. Ahmad Ibn Hanbal (780 - 855), for example, was seeing that the weak Hadith is stronger than the mind's opinion, while the people of opinion, with the leadership of Abu Hanifa (699-767), were suspecting in Hadith if they feel that it is abnormal, and then leave it to the opinion and ijtihad.

However, the fruit of intellectual tolerance and mutual respect between the two schools was that God made easy for them the meeting in a middle position at the hands of Imam Shafi'i and his students. Since it became necessary for the 
people of Hadith to be armed with the opinion, and for the people of opinion to be armed with Hadith. ${ }^{49}$

We should not think that the jurisprudential schools had been confined to the four schools known today, namely: Hanafitism (a reference to Imam Abu Hanifa), Malikitism \{a reference to Imam Malik Ibn Anas $(715$ - 796)\}。 Shafi' itism (a reference to Imam al-Shafi'i), and Hanbalitism (a reference to Imam Ahmad Ibn Hanbal). Nevertheless, the freedom guaranteed by the State for the judges and the people regarding the fatwa has led to multiply the jurisprudential schools, until they reached in the third century to seventeen schools. ${ }^{50}$

However, most of these schools could not survive; either because of external circumstances, such as lack of strong students, who can help spread the doctrine and defend it; or for internal reasons. An example for the latter is the case of the doctrine of al-Zahiriyah [a reference to Abu Dawud alZahiri (d. 882)], which has ended because of its inflexibility, its rejection of the opinion, and its adherence to the text. Thus, the schools of jurisprudence became today, for the Sunni sects, the four known schools only. ${ }^{51}$

The door of ijtihad has been closed in the end of the fourth century because of the weakness of Abbasid state, the corruption in everything, even the jurisprudence and the judiciary, and the fear - which continues to this day - that the methods of deduction will become a way to satisfy the rulers by the formal opinion in their favor. But that does not mean that the creative energy of jurisprudence have been exhausted and consumed. It does not mean either that the process of interpretation of the texts had been completed at the hands of the scholars with the unique capacity, whose efforts led to formulate Sharia in its final form.

In other words, the closing of the door of Ijtihad does not mean the sterility of Islamic state to procreate the qualified scholars (al-Mujtahidun), and limiting the activity of jurists in the tradition of previous doctrines, or limiting the Islamic 
legislation to codify the judgments established in advance and formulating them in an educational form. On the contrary, the Islamic nation is rich with its scholars and jurists; the evidence is the Prophet's saying: «God sends to this nation at the top of every one hundred years who renews its religion». Therefore, the big names, which we can not ignore, appeared in the Jurisprudence, such as al-Ghazali, Ibn Taymiyya, and alShawkani. ${ }^{52}$

Today we are in dire need of persons who can renew the nation's religion by ijtihad and opinion, especially after Muslims faced many eternal and external problems and issues that were not known, but could not be imagined by our ancestors. Is there any way to do that?

At the outset, it should be noted that we do not mean by renewal those blatant sayings that resound today by some and which are not clear from the political purposes - to inspire the Western culture and surrender to its demands in order to keep pace with the age. The West, with its successive pressures on the Islamic governments, and its blatant intervention in their different affairs, only aimed to clear the religious text from what may be opposed to its political and economic interests. It aims also to restructure the system of Arab-Islamic culture in a framework of the values and priorities that ensures the retardation of Islamic Arab mind, hence the absolute hegemony. We do not mean either by renewal those calls by some to revive the jurisprudential literatures of the ancestors, besides the historical and methodological significance of them, in order to stop at what they contained, and comply with them literally. Our ancestors had their own daily problems with the specific local variables, while we face now new daily problems, which the ignorance of religion motivates us to seek their solutions at the others. We mean rather the awakening of the Arab-Islamic mind from its dogmatic slumber, which retreated with it to the back of the civilizational riders in all areas, and to revive the spirit of mental courage shown by the ancestors, then they break 
through the corridors of forensic and natural science without stress or duplicity or procrastination. We should not argue about the contention that the unanimity of nation had been held on closing the door to ijtihad. We can satisfy in this regard with the view of Imam Ahmed Ibn Hanbal and the Salafi doctrine that the unanimity of Islamic nation was not achieved since the rift that hurt Muslims in the era of the third Caliph Uthman Ibn Affan (580 - 656).

Moreover, we should not take the fear of the following of passion as a reason for the permanence of closing the door to ijtihad for more than ten centuries; the matter is committed to the consciences of scholars and jurists. Nevertheless, it should be recognized that the submission to the influence of West (Europe and USA) by much of the Muslim rulers would remain a source of concern and uncertainty in the fatwas from which the odor of politics is smelled.

Finally, given the complexity of contemporary issues and their distribution among astronomy, medicine, law and economics, as well as morality and aesthetics, there is no room for the individual absolute ijtihad in our time, in the sense that the problems of today are not as those in the past time, which can be solved by one jurist. But the efforts of scientists and experts from various fields must be combined to reach the religious opinion that is suit for the variables of our era. That requires that the jurist, or the jurisdiction, have experts in the various disciplines, should be consulted, and they must be, naturally, from the people of piety and devoutness, in addition to their expertise and specializations. The conference should convene to consider the issues and problems that concern the people, and discuss these issues by every interested party of scientists, experts and university professors, as well as jurists, then submit the recommendations of the conferences to the jurisdictions, so that the final determinations are not confined inside offices or public libraries. ${ }^{53}$ 


\subsection{Non-Aristotelian Logical Elements in IIm Al- Kalam}

The science of the principles of jurisprudence (ilm usul alfiqh) is not the only field of research, in which the Muslim thinkers violated the basics of Aristotelian logic and got going to set up their own logic, which is appropriate to the needs of their religion and the variables of their life. In fact, there is another important and fertile field, in which the Muslim disagreed also, explicitly and clearly, with the Aristotle's three laws of logic, in particular the laws of Non-Contradiction and Excluded Middle; namely the field of ilm al-Kalam.

These laws - as stated - are the basis of the Aristotelian logic. Therefore, this logic is called sometimes 'the science of thought's laws', which reveals the danger of such laws in the building of the Aristotelian system, and its control over different logical systems for many centuries. Aristotle, as well as their carriers to Islamic thought, regarded them as general and intuitive principles that make the logic a genuine science, or the science of sciences.

The first law (the Principle of Identity) states that everything is itself, or 'if there is a true proposition, then it is true'. In a symbolic formula:

\section{$\boldsymbol{P} \supset \boldsymbol{P}$ or $\boldsymbol{P} \equiv \boldsymbol{P}$}

The second law (the Principle of Non-Contradiction) states that one cannot say of something that it is and that it is not in the same respect and at the same time. In the symbolism of propositional logic, this is expressed as:

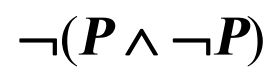

This law is complementary to the first, because except for what it supplies to us, the principle of identity, on the one 
hand, will become not complete from the logical side. On the other hand, it will be not understood exactly. According to some logicians, this law does not go beyond the law of identity as both of them states, in two phrases that are opposed in the form not in the essence of meaning, that the truth is one.

The third law (the Principle of Excluded Middle) states that there is no middle between two extremes, or that any proposition can be only true or only false and there is no a third between them, namely:

\section{$(\boldsymbol{P} \wedge \neg \boldsymbol{P})$}

Before the West's thinkers paid attention to the need to exceed these laws in response to the requirements of reality, and in recognition of the essential features of our knowledge; such as vagueness, probability, and uncertainty... etc., the thinkers of Islam had already exceeded them in response to the requirements of interpretation, and the building of the mental argument. That was in the field of ilm al-Kalam.

Let us offer models of these problems that indicate the contravention of the Kalam's scholars to the laws of Aristotle's logic, albeit indirectly, and the correspondence of their logical understanding to the principles of neutrosophic logic, particularly the possibility of combining the two extremes or the presence of a third middle between them.

\section{A. The judgment on the Perpetrator of a Major Sin}

The judgment on the perpetrator of a major sin (Murtakib alKabirah) represents a fixed one of the five principles of Mu'tazilah; namely the principle of the position between two extremes. Some historians argue that this judgment, from the historical point of view, is the first starting point of the Mu'tazilah as a religious movement. ${ }^{54}$ In Islam, the perpetrator of a major sin is that the adult and sane Muslim who perpetrates a sin that is prohibited unequivocally by theQuran and Sunnah, and Allah threatened its doer with hell. Some 
examples of these sins are polytheism, leaving prayers, disobedience parents, usury, magic, adultery, deprivation anyone of his life, which Allah has made sacred, unless it be justified for a proved crime, eat the wealth of the orphan, and others up to seventy forbidden deeds. ${ }^{55}$

How we judge logically on the perpetrator of a major sin? Do we say that he is 'infidel' (regardless of the serious consequences followed from this judgment, such as the prohibition of his marriage of a faithful woman, or separating them if he has been married to her), although he speaks the Basic Statement of Faith for becoming a Muslim? ${ }^{56}$ Or must we let his affair to Allah in order to judge on him in the Day of Resurrection as He wishes, even though he declares the major sins in the Muslim community?

This question may not be, at first glance, outside the religious framework that does not lead to widening the gap between $<\mathrm{a}>$ and $<$ anti- $\mathrm{a}>$, but it took, for the Islamic schools, an important political dimension; Kharijites were opposed to the Umayyad State, and they wanted to justify their war and secession on an ideological basis.

The Umayyad Caliphs, except the seven one Omar Ibn Abdal-Aziz (680 - 720), were not worthy, from the side of religious preference, to be emirs of the believers. They also came to the authority by force, but they did not refrain from committing the major sins such as bloodshed and exaction of the fortune. Therefore, Kharijites declared that the perpetrator of a major $\sin$ is infidel, so that they can fight him. But another group of Muslims, Al-Murji'a or Murjites (=the postponers), troubled by the bloodshed and the continued wars among Muslims, and despaired of the disposal of the Umayyad Dynasty, argued that the perpetrator of a major sin is believer, and that the major sins committed by him are not incompatible with the belief in his heart. Therefore, we must defer the judgment on him till the Day of Judgment; if Allah wills, He will punish him; and if He wills, He will forgive him. In front of this extreme position, the sect of al-Mu'tazilah 
appeared to declare that the perpetrator of a major $\sin$ is neither a believer nor a disbeliever, or he is a believer and unbeliever at the same time. In other words, he is in a position between two extremes, because he resembles the believer in his tenet, not in his deed, and he resembles the disbeliever in his deed, not in his tenet. Thus, $<$ a $>$ and $<$ anti- a $>$ meet in a middle position that combines them, but they battle through the infinite distance between them. This means that the faith increases and decreases continuously, then the degrees of believers vary according to the variedness of their deeds. However, if the perpetrator of a major sin leaves the life without repentance, he will be one of the Hell's people, wherein he shall last forever, as in the Hereafter there are only two parties; one shall be in the beautiful and blissful region of heaven's realm and the other in the blazes. Nevertheless, the torment of the perpetrator of a major sin will be less, and his abode in the Hell will be over the infidel's abode.

This Mu'taziah's opinion does not include any inclination to the Kharijites' opinion, but it is fully consistent with the Mu'taziah's understanding of the meaning of faith; the faith is not only the belief of heart or the pronunciation of tongue, it must be confirmed by the pious act. Therefore, the perpetrator of a major sin deprived of the faith when he did the evil. Moreover, his departure from the life without repentance means that he deserves of the Immortality Penalty. ${ }^{57}$

\section{B. The Mental Goodness and Badness of Actions}

Mu'tazilah faced different religions that challenge the absolute unity and oneness of God (Wahdaniyyat Allah) in the pure Islamic sense; whether this challenge through anthropomorphism (Judaism), Trinity, (Christianity) or Dualism (Manichaeism, Zoroastrianism, and others from the Persian religions). If the absoluteness of $\mathrm{God}$ in the Mu'tazilah's principle of monotheism (al-Tawhid) came in response to the Jews and the Christians, their concept of the Divine act, in the principle of Divine justice (al-Adl) came in 
response to the schools of Dualism. The Dualism says that there are two Gods; the God of Light, from whom the goodness emanates, and the God of Darkness, from whom the evil emanates. Then, the question that faced Mu'tazilah was the following: How can one explain the existence of evil in the world without compromising on the absoluteness of God on the one hand, and His wisdom and justice on the other? In other words, if there is no way to deny the existence of evil in the world, can the evil emanate from wise and omniscient God? To answer this question, Mu'tazilah used the two terms: 'Goodness' and 'Badness' of actions (Al-husn wa al-qubh alaqliyyan), and they set a crucial distinction between them and the two terms 'benefit' and 'adversity' (Al-naf'a wa al-dur). Anything that we say that it is beneficial is not good necessarily, and anything that we say that it is harmful is not bad necessarily, but what is harmful or painful may be good, and what is beneficial or pleasurable may be bad. The man may seek pain if its benefit is more than its adversity, as the compassionate father when he cultivates his son by depriving him from what he may desire. In addition, the pains and disasters are not bad when they are tests for man, because he will deserve reward if he can be patient with them. This is expressed by Allah's saying:

If Allah were to give livelihood generously and liberally to all His creatures and to confer support gratuitously, they would be apt to run riot on earth and transgress the goal. But He sends down to the extent He prescribes as He will; He is Khabirun (Omniscient), and Bassirun (Whose constant vigilance is extended to all His creatures.)

(Counsel $=$ Al-Shura, verse 27)

This means, as Abu al-Husayn al-Khayyat (d. 951) stated, that Allah, to whom be ascribed all perfection and majesty, really is the causer of illness; i.e., no one ill himself; He is also the inflictor wilting on the plant, etc. However, nothing of that is considered evil or corruption; one may hate these things, but 
some of them are not bad. The bad is what is pure evil or pure vanity, but all that is impossible for God. ${ }^{58}$

On the other hand, if the Divine Justice requires that acts of God be designed only for what is good, the judgment on the act that it is good or bad is due to the characteristics of the act itself, not only because God ordered us to do it or prohibited us from do it. Allah ordered us to say the truth because it is good, and prohibited us from lying because it is bad, and the human mind is capable of detecting the aspects of goodness and badness in each act. The minds are not differing in the distinction between the goodness and badness of acts, though they are differing in details, as Kharijites are approving to kill those who differ with them, while most sects of Muslims are seeing that it is a bad act. However, if the acts are approved or condemned for themselves, this does not mean that moral judgments, or mentalities, are absolute, so that we say that there all truth is good, and all lying is bad, since there is nothing of the acts could continue on one condition, and in all circumstances. In the language of neutrosophic logic, we can say that $<\mathrm{A}>$ tend always to be $<$ Not-A $>$, including the $<$ Anti$\mathrm{A}>$, then, a particular lying may be approved, and a particular truth may be condemned. Two identical acts may result form two different reasons, then that requires the difference of two acts; for example, the killing sinfully and wrongfully is not the same as the killing in the case of punishment or retribution. The act may also differ from one case to another because of the difference of time, then the bad becomes good and the good becomes bad. As well as the act may differ from one case to another because of the difference of abilities or the difference of actors. Therefore, we must estimate the difference of position, time, cause, and ability, because of this difference the act becomes another act. ${ }^{59}$

\section{The Somethingness of Nihil}

The Theory of somethingness of nihil, attributed to Abu alHusayn al-Khayyat, caused much controversy among scholars, 
and because of it, the Mu'tazilah accused many accusations that are far from their destination, and in which the truth lost in the dust of opponents' interpretations.

The logical starting point for this theory (or problem) is a verbal argument about the meaning of the word 'something' in the language; it is a word abounds on our propositions that reflect our knowledge of the world, and it has been mentioned in the Quran 192 times to serve different connotations.

Perhaps the simplest and most likely linguistic definition of the word «something» that «what could serve as a subject in a proposition» (i.e., the detective assigned to him or him). But this definition leads to the following logical question: is the predication in the categorical proposition means the concrete existence of its subject? In other words, does the logical copula include the existence? or its function is limited to mere predication? To answer this question Muslims split into three different sects, corresponding to three known philosophical tendencies. These sects are: ${ }^{60}$

1. Anthropomorphists (=Al-Mushabiha): those who do not verify the predication but to what is a sensible body. This is the position of sensualism (or sensationalism) in philosophy.

2. Ash'arites (Al-Ash'ariyyah): those who do not verify the predication but to what is existent actually. This is the position of realism.

3. Mu'azilites (Al-Mu'tazila): those who broaden the circle of predication to include the concrete existence on the one hand, and the mental one on the other. This is the position of rationalism.

Thus, if there is an Islamic agreement on the possibility of describing God Almighty that $\mathrm{He}$ is «something», based on the Quranic verse:

Ask O Muhammad those who doubt your mission: «What documentary evidence has more weight to support the truth of my mission than the attestation by Allah 
Himself!» «He is the witness of my actions and of your actions»

$$
(\text { Cattle }=\text { Al-An'aam, verse 19) }
$$

[Where the 'documentary evidence' is equivalent to

'something' in Arabic language]

The meaning of that, according to anthropomorphists, that God is a body. And according to Ash'arites, it means that God is existent actually. But the meaning of 'somethingness' for Mu'tazila is the predication, where the latter is more general than the concrete existence.

The anthropomorphists' position is based the Quranic verses that mean externally the anthropomorphism (= al-Tashbĩh), but Mu'tazila have interpreted them in a manner consistent with their unlimited absoluteness of God. As for Ash'arites, they have evidenced from the Quran that the concept of 'somethingness' requires the existence, namely by the verse: Or have they been created by chance, or are they the creators or supreme beings who created all things? (The Mount = Al-Tur, verse 35); since the «non-thing» points out the nihil (or nothingness = al-Adam), the «thing» indicates the existence (= al-Wujud); also the verse: "«So be it», says Allah, your Creator; «this is an easy act actuated upon My swill and I have created you earlier when you were nonthing!» (Maryam = Mary, verse 9), i.e., 'when you were non-existence'. As for Mu'tazila, they have evidenced by more than Quranic verse in supporting their concept of 'somethingness'. The first verse, to which they always refer to indicate the unlimited absoluteness of God, is:

Nothing material or immaterial is like unto Him (Al-Shurah $=$ Counsel, verse 11)

This verse does not only indicate that there is no thing like God, But God is different from everything that is imaginable or perceptible. In addition, Allah's saying: Allah, My Creator is Omnipresent, and His Omniscience embraces all in all $\left(A l-A n^{\prime}\right.$ aam $=$ Cattle, verse 80$)$, and His saying: Fnd that His Omniscience covers all things in all 
respects $($ Al-Talaq $=$ The Divorce, verse 12). The 'somethingness' here not refer only to the concrete existents, but also to everything that we agreed to call the nihil, such as the Day of Judgment; it is nihil in the past and present, but it is known to God.

On the other hand, if the «thing», according to Ash'arites, is synonymous with the existent, then the nihil (or nothingness) means necessarily the 'non-existent', but Mu'tazila understood the 'thing' as the proposition's subject; that is a synonym for both concrete and intellectual existent. The latter contains possible and impossible; what already exists and what only exists as a notion or idea. No place then for the law of noncontradiction or the law of exclude middle, but the two extremes may meet, therefore we can say that the nihil or the 'nothingness' is 'something', as it is a truth or self-quality known to the Almighty Creator before it realizes in the concrete existence by His command. There is no room here also to say that the world is eternal, as the natural field of the theory of 'somethingness' is epistemology, not ontology. The sense that the mental perceptions, in terms of that they are perceptions, are simply mental necessary propositions, which are separated form the quality of existence. Furthermore, the philosophers who say that the world is eternal do not prove the possibility of existence of nihil, because things have not come into existence from pure nihil, but from a first matter. As for Mu'tazila, they had proved the nihil side by side with what is possible to be existent in terms of the God's ability to create it, which confirms that they believe in the Islamic theory of creation. This is reflected in the Quranic verse: Whenever it is His will to bring, anything (animate or inanimate) into being, all it takes is to say «Be» and «it is» (Yaseen, verse 82); i.e., from the nihil to the existence by virtue of His saying 'Be'. Also there is no room to say that the Mu'tazila's conception of the nihil is corresponding to the Plato's conception of the world of ideals, because the latter means that the mental things have an external existence; hence, the 
ideals are relating to ontology, but there is no thing of that of Mu'tazila $^{61}$

\section{Theory of Modes (or States)}

Theory of modes (Al-Ahwal) relates to the theory of somethingness of nihil in terms of their separation between what is in the reality and what is in the mind, as well as their revolution on the law of excluded middle and their saying that, the two extremes may meet. Both of them is an evidence on the fully consistence in Mu'tazila's mental tendency.

This theory is attributed to Abu Hashim al-Juba'i (d. 933), but his father Abu Ali al-Juba'i (d. 915) opposed it. The theory originally arose as a reaction to the Christian viewpoint, which is contrary to the Islamic point of view, on the relationship between the God's Attributes (= Sifat Allah) and His Divine Essence (= Dhat Allah). In other words, the Mu'tazila wanted, by this theory, to refute the idea of Embodiments (= alAqanim) or trinity (= al-Tathlith) in the Christian religion, as this idea argues to that Allah is one substance with three embodiments: Existence (= al-Wujud), Awareness (= al-Ilm), and Life (= al-Hayah). These Embodiments at the beginning were attributes, and then became persons; The Father $(=$ al$A b)$, The Son (= al-Ibn), and The Holy Spirit (= al-Ruh alQudus). Since these Embodiments are separated from each other, it was possible that the second Embodiment (the Awareness or the Word) to be embodied in Jesus (= AlMasih), then He became unlike The Father.

No doubt that this idea is fully contrary to the Islamic doctrine of monotheism (al-Tawhid), which Mu'tazila wanted to purify from any blemish or defect, then they argued that God's Attributes are the same as His essence, not additional to it. Mu'tazila's thought has been controlled by the necessity of excluding any concept that can mean plurality in the Divine essence; Awareness, Life, and Ability, as attributes, are the same of essence, meaning that there is an identity between them and the essence, so that this identity prevents their 
independence from the essence. In this way, the Mu'tazila went beyond the problem of quantity (the pure monotheism), but at the same time they faced the problem of quality. If Allah Has an awareness, and His awareness is His essence; Has an ability, and His ability is His essence; Has a life, and His life is His essence, then what is the difference among awareness, ability, and life?

The initial Mu'tazila's answers to this question characterized by confusion and hesitation; they said at times that the difference is reflected in the dissimilarity between the known and the affordable; other times they said that it is realized according to the difference of antonyms of these attributes. Nevertheless, the problem remained outstanding; how these antonyms are differing in the same time that the attributes itself are not differing? In addition, Mu'tazila faced another Muslim group that accused them of denial - or disruption the attributes; those are Al-Sifatiyyah (= Attributists). Then, Mu'tazila had to take a decisive position through which could face the attributists on the one hand, and maintain the pure monotheism on the other. At this time, «Abu Hashim» presented his theory of modes as a compromise and acceptable solution to this problem. He said, if the essence of God is not a cause of His awareness and ability, because the concept of essence is differing from the concept of awareness or ability, it is possible to say that these attributes are not but «known» modes merely. This means that the attributes are mental considerations (which are a subject of awareness), while the Divine essence is only one substance (which is a subject of concrete existence). In this way, the mode can be defined as it is every attribute of a being that is not fall within the topic of existence; i.e., the mode is relating only to the topic of meaning, not to the topic of existence. In other words, we can say that the modes are mental concepts, which are not described as they are existing or not existing, because the existence and nothingness relate only to the world of objects. Ruther, the modes are described as predicated, because they 
relate to the world of minds. That, undoubtedly, is consistency with the Mu'tazila's saying concerning the somethingness of nihil.

Thus, Abu Hashim proved that there are attributes of the Divine essence, stressing the diversity of their notions, and excluding their existence to avert the suspicion of plurality. To illustrate this sense, he divided the attributes into two classes, which are: ${ }^{61}$

1. The attributes that are predicated to the essence according to a cause; the awareness and ability, as well as hearing and vision, for example, are attributes conditioned by the life, and they are caused by life.

2. The attributes that are predicated to the essence without cause; such as the distinction of substance, and the fact that the accident is accident, and the blackness is blackness. These attributes, in our modern expression, are the propositions in which the predication does not add a new thing to the subject.

The God's attributes, in terms of their connection with the essence, not of the second class, because it does not fall under the concept of «essence» that the God, for example, is capable. However, these attributes are not known or recognized as separated from the essence, just as we do not recognize the being's hearing and vision independently of that he exists or live. Then, the attribute is not the essence itself; nevertheless, it is not achieved without the essence. Perhaps the best expression of this fact is Al-Tahawi's saying: «If you said: I commit myself to Allah, then you committed yourself to the Holy essence described in the attributes of completeness, which are attributed to the God Almighty, and which is inseparable from Him in a sense. And if you said: I commit myself to Allah's glory, then you committed yourself to one of Allah's attributes; nevertheless, you did not commit yourself to anything but Allah. This meaning is understood from the term dhat (essence); al-dhat, in its original Arabic 
meaning, is not used but as added; i.e., dhat wujud (having existence), dhat qudrah (having ability), dhat ilm (havind awareness), dhat izah (having glory), etc. The Prophet Muhammad said: I commit myself to Allah's glory and ability counter to the evil generated by what I face, I commit myself to Allah's perfect words counter to the evil generated by His creatures. Nevertheless, the Prophet did not commit Himself to anything but Allah». ${ }^{62}$ 


\section{Notes of Chapter 3}

1. This chapter is written by Salah Osman.

2. See:

- Rescher, N., Many-Valued Logic, McGrow-Hall, N.Y., 1969.

- Salah Osman, Many-Valued Logic between the Degrees of Truth and the Limits of Knowledge (Al-Mantiq Mut'adid al-Qiyyam bayna Darajat al-Sidq wa Hudud al-M'arifa), Establishment of Knowledge (Munsha't al-Ma'arif), Alexandria, Egypt, 2002.

- Williamson, Timothy, Vagueness, Routledge, London \& N.Y., 1994.

- Salah Osman, Dialectic of Constancy and Motion in Zeno's Paradoxes: a Contemprary Mathematical View (Jadal alThabat wa a-Haraka bayn Darjat al-Sidq wa Hudud alM]arifa), Journal of Facult of Arts , Menoufia University, Egypt , (58), July 2004,pp. 99 -139.

3. Hassan Abdel Hamid \& Muhammad Mahran, On the Philosophy of Science and methodology (Fi Falsfat alUlum wa Manahij al-Bahth), Said R'afat Library (Maktabat Said R'afat), Cairo 1979 / 1980, p. 68.

4. See chapter one, $\S 1.4$.

5. 'Testimony' is a declaration by a witness under oath, as that given before a court or deliberative body. Islam emphasized the testimony in the matters of daily treatments and transactions in order to ensure justice based on the evidence, and prevents the digestion of people's rights, as is the case, for example, in affairs of liability (taking money 
in advance), selling, and others. The testimony of two men is confirmed by the Quranic text:

O you with hearts touched by the divine hand, if you become involved in a debt payable at a fixed date, be it a loan, item borrowed or bought on credit or a business transaction, then commit it to writing. A scribe shall formulate an agreement or contract mediating the parties with recourse to the general principles of Allah's justice. No scribe shall refuse to fulfil this function as Allah imparted it; he simply writes and the debtor dictates and keeps Allah in mind, and the scribe shall not decrease any of the agreed amount. Should the debtor be of unsound mind, weak, or incapable of dictating, then his guardian should dictate according to equity and good conscience. And select two of your men to be formally present as witnesses to the transaction. And if two men are unavailable, then one man and two commendable women so that later, if one errs due to a memory lapse, lack of experience or a biological factor, then the other will remind her of the correct testimony. And those who were called upon to witness the transaction and were associated with the event must not refuse when they are called in for evidence in the future. And do not be disinclined to commit the debt, small or large, to writing. Sanctioned by Allah according to judicial principles, this is recourse to a credited testimony preventing doubt. This is mandatory unless it is business involving bartered merchandise or collective management at an immediate cash rate, then you are absolved if you do not commit it to writing. But when you dispose of merchandise to a buyer for price, then such transactions must be witnessed. And neither writer nor witness should be made to suffer, for if you harm them, you commit a sin that shall be transferred to you, and revere Allah; He teaches you and shows you 
through information or instruction; He is 'Alimun of all in all

$$
\text { (Al-Baqarah }=\text { The Heifer, verse 282) }
$$

Islamic law (Sharia) has specified some conditions of the witness, including 'justice': You must bring two of your people, who are known to recourse to general principles of justice $($ Al-Talaq $=$ The Divorce, verse 2$)$, 'puberty', 'Islam', 'freedom', and 'non indictment'.

6. 'Penance' or 'expiation' is an act of self-abasement, mortification, or devotion performed to show sorrow or repentance for sin. There are many types of it in Islam, such as the penance of the perjury (the oaths in which guilt resides in the intention), of the failure to perform some rites of pilgrimage (Hajj), and of manslaughter. With regard to the latter, it was identified by the Quranic verse:

Observe particularly: It is not right for a believer who has conformed to Islam to kill another believer of the same law unless it be by mistake. And he who kills a Muslim by chance and guilt does not reside in the intention shall run into some consequences in expiation of his wrong. Thus: a) The release of a Muslim slave from bondage and the restoration of his freedom and personal rights, in addition to the payment of blood money to his next of kin as a compensation for the loss of a relative, unless they remit it of their own accord in benevolence. b) If the slain be a believer who has conformed to Islam but his people are an enemy to faith, then you incur only the release of a Muslim slave from bondage and the restoration of his freedom and personal, rights. c) And if he is a Muslim whose people stand friendly with you and their is a binding agreement in which you both enter, then you incur the payment of the blood - money and the release of a Muslim slave from bondage and the restoration of his freedom and personal rights. d) And he who does not have the means to fulfill the divine law as precisely 
indicated shall have to fast- dawn to sunset- for two consecutive months in expiation of sin; Allah is ever 'Alimun, and Hakimun

(Al-Nisa $=$ The Women, verse 92)

As for the murder, Allah made its punishment very great: And he who kills a believer who has conformed to Islam and the guilt resides in the intention, shall be destined to Hell wherein he will have passed through nature to eternal suffering and he will have come within the measure of Allah's wrath and curse. This is besides the torment prepared by Providence to lay upon the damned

$$
(\text { Al-Nisa }=\text { The Women, verse 93) }
$$

However, Islam has left the door open for those who wanted to repentance, unless the soul reaches to the last moment of death. The Prophet Muhammad expressed this in his saying: "Allah is accepting the repentance and the atonement made by His servant unless the soul is on its way out and it makes a rattle in the throat». If the guilt was between one and God, i.e., not relates to the right of a human being, then the repentance has three conditions; a) He must abandon the guilt, b) He must regret that he had done it, and c) He must determine to return to it at all. On the other hand, if the guilt is related to the right of a human being, a fourth condition must be added. That is he must acquit from this right, so if the retaliation has not done by the victim's next of kin, and they accept to take the payment of blood-money as a compensation for the loss of a relative, the repentance becomes necessary.

7. The testimony of al Fasiq (shameless sinner) is unreliable according to the Quranic verse:

Actions based on false reports must not be taken until one is absolutely certain that what has been reported is true. Otherwise one may wrongly discredit or disgrace someone or injure his or her reputation and this is utterly forbidden 
(Al-Hujurat $=$ The Chambers of The Messenger's Wives, verse 6).

8. Abd Allah Ibn Ahmad Ibn Qudama al-Maqdisi, The Garden of Looker and the Paradise of Spectacles (Rawdat al-Nadhir wa Janat al-Manadhir), ed. By Abd al-Aziz Abd al-Rahman Said, University of Imam Muhammad bin Massoud, Riyadh, second edition, 1978, Vol. 1, pp.293-94.

9. Abd al-Karim Zidan, Summary of the Fundamentals of Jurisprudence (Al-Wajiz fi ilm usul al-Fiqh), The Islamic House of distribution and publishing (Dar al-Tawzi'i wa alNashr al-Islamiyah), Cairo, 1993, p. 195.

10. Abu Ishaq Ibrahim Ibn Ali al-Shirazi, The Bright Light in the Fundamentals of Jurisprudence (Al-Luma' fi Ilm Usul al-Fiqh), ed. By Yusuf Badeiwi \& Mohi al-ddin Dib Mostawi, Ibn Kathir House (Dar Ibn Kathir), Beirut \& Damascus, second edition, 1997, Vol. 1, p. 52.

11. Imam al-Shafi'I, Al-Risalah for the authoritative position of the Sunnah, ed. By Ahmad Muhammad Shakir, The Scientific House of Books (Dar al-Kutub al-Ilmiyyah), Beirut, 1992, Vol. 1, p. 476.

12. Abu Hamid al-Ghazali, The Chosen in The Science of the Origins of Jurisprudence (al-Mustasfa), second edition, The House of Scientific Books (Dar al-Kutub al-Ilmya), Beirut, 1983, Vol. 2, p. 229.

13. This Quranic verse refers to the punishment of adultery only for the non-married, but for the married, he (or she), will be pelted with stones up to death in order to find the pain in all parts of the body. The stoning here is equivalent to that he (or she) has demolished a house of family. It is known that the punishment in Islam has some conditions to be achieved, namely: puberty, reason, awareness of prohibition, and free will.

14. See Abd al-Karim Zidan, Summary of the Fundamentals of Jurisprudence, Op. Cit, pp. $403-404$.

15. Imam al-Haramayn Ibn al-Juwayni, The Proofs of Jurisprodence (Al-Burhan fi Usul al-Fiqh), ed. by Abd al- 
Adhim al-Dib, The House of al-Ansar (Dar al-Ansar), Cairo, 1980, Vol. 2, p. 485.

16. Abd al-Karim Zidan, Op. Cit., p. 197.

17. Imam al-Shafi'I, Op. Cit., pp. $509-511$.

18. 'Interpretation' in the idiom of the Islamic jurists and scholars is the transformation from a likely meaning of the term to a more likely meaning. For more detail, see, Abu Hamid al-Ghazali, Law of Interpretation (Qanun al-Ta'wil) (read and left the talks and commented upon by Mahmoud Peugeot, House of Minds (Dar al-Albab), Damascus, 1993, p. 7.

19. See Abu Hamid al-Ghazali, Al-Mustasfa, Op. Cit., Vol. 2, p. 325. Also Muhammad Ibn Ali al-Shawkani, Guidance of the Masters to the Realization of the Right of the Science of Origins, (Irshad al-Fuhul ila Tahqiq al-Haqq min Ilm alUsul), ed. by Ahmad Inayyah, The House of Arab Book for Publishing and Distribution, (Dar al-Kitab al-Arabi li alNashr wa al-Ta wzi') Beirut, 2002, Vol. 1, p. 304.

20. Al-Ghazali, Al-Mustasfa, Op. Cit., Vol. 2, pp. $325-26$.

21. Abd al-Karim Zidan, Op. Cit., pp. $198-200$.

22. For example, the Quranic verse:

The forbidden articles of food are being named: Forbidden unto you are carrion and blood, flesh of swine and flesh of the animal found strangled and the slaughtered under the invocation of a name other than Allah's Name, flesh of an animal beaten to death and the one that died by a fall, the gored to death and the victim to a carnivore unless it be slaughtered in time, and the flesh of the animal sacrificed on the altars (idolism)... But he who is forced, by reason of hunger and want of lawful food, to eat of the forbidden, and not by willful transgression of Allah's commandments nor does disobedience reside in the intention, shall find Allah Ghafurun (Forgiving) and Rahimun (Merciful)

$($ Al-Maidah $=$ Heaven's Sent Festive Table, verse 3) 
Thus, who is forced in a famine can eat these forbidden articles of food, and we can measure on this case the illness or any other need, where the eating of these articles of food become lawfulness.

23. Khuzaymah Ibn Thabit (a Companion of the Prophet), was called by the Prophet 'the owner of the two testimonies'. The story of this nickname is that the Prophet bought a horse from a Bedouin, and He took him with Him to pay him the price of his horse. The Prophet walked quickly and the Bedouin walked slowly. The people stopped the Bedouin and began to bargain with him for the horse as and they did not know that the Prophet had bought it. The Bedouin called the Prophet saying 'If you want this horse, then buy it, otherwise I shall sell it'. The Prophet stopped when he heard the call of the Bedouin, and said 'Have I not bought it from you?' The Bedouin said 'I swear by Allah, I have not sold it to you'. The Prophet said 'Yes, I have bought it from you'. The Bedouin began to say 'Bring a witness'. Khuzaymah ibn Thabit then said 'I bear witness that you have bought it'. The Prophet turned to Khuzaymah and said 'On what (grounds) do you bear witness? '. He said 'By considering you trustworthy, Apostle of Allah!' The Prophet made the witness of Khuzaymah equivalent to the witness of two people.

24. See Al-Ghazali, Al-Mustasfa, Op. Cit., Vol. 2, pp.330-31, also Badr al-Din Muhammad al-Zarakshi, The Surrounding Sea in the fundamentals of jurisprudence (Al-Bahr alMuheit fi Usul al-Fiqh), ed. by Muhammad Muhammad Tamir, The House of scientific books (Dar al-Kutub alIlmiyyah), Beirut, 2000, p. 136.

25. See Muhammad Ibn Abu Bakr al-Razi, The Chosen of Correction Dictionary, (Mukhtar al-Sahah), ed. by Mahmoud Khater, The House of Hadith (Dar al-Hadith), Cairo, without date, item 'Cause' (Allala), p. 451.

26. Abd al-Karim Zidan, Op. Cit., pp. 203 - 204. 
27. Ahmad Mahmoud Subhi, This is My Book, Read it (Ha'um Iqra'u Kitabiyyah), Library of Arab Raising (Maktabat alNahda al-arabiyyah), Beirut, 1997, p. 159.

28. See:

- Muhammad Ibn Ali al-Shawkani, Guidance of the Masters to the Realization of the Right of the Science of Origins, Op. Cit., Vol. 1, p. 304.

- Ahmad Mahmoud Subhi, This is My Book, Read it, pp. 159 -60 .

- Abd al-Karim Zidan, Summary of the Fundamentals of Jurisprudence, Op. Cit., pp. 205 - 208.

- Ali Sami Al-Nashar, Methodology of the Islamic Thinkers and the Discovery of the Scientific Method in the Islamic World (Manahij al-Bahth ind Mufakiri al-Islam wa Iktishaf al-Minhaj al-Ilmi), The House of Knowledge (Dar alMa'arif), Cairo, 1967, pp. 110-113.

29. Islam denied the marriage of some relatives in order to preserve the family life, to realize the social communication, to avoid grudges, and to keep the lineal descent, which is reflected in the Quranic verse:

Forbidden Degrees: Certain degrees of relationship within which people are forbidden to marry. Forbidden are: your mothers, your daughters, your sisters, your paternal and maternal aunts, your nieces on the brother and sister's side, your foster-mothers, your fostersisters, your mothers-in-law, your stepdaughters with whose mothers you have consummated marriage. But you are absolved to marry them if you have annulled your unconsummated marriage to their mothers-your daughters-in-law whose husbands are your own sons who generated from your own loins. Nor can you effect a, matrimonial union with two sisters at the same time; bygone shall be bygone; the new era shall begin; indeed Allah has always been Ghafurun (Forgiving) and Rahimun

$($ Al-Nisa $=$ The Women, verse 23) 
It is clear from the two preceding verses that the taboo women are three types:

A) The taboos because of descent, they are: mothers (including grandmothers), daughters, sisters, paternal and maternal aunts, nieces on the brother and sister's side, foster-mothers, foster-sisters, mothers-in-law, stepdaughters with whose mothers you have consummated marriage.

B) The taboos because of sucking, they are: fostermothers and foster-sisters. Sunnah explained that the taboos because of sucking are seven articles, as the case of the descent.

C) The taboos because of the marriage, they are: mothers-in-law, stepdaughters with whose mothers you have consummated marriage, wives of sons, and wife' sister.

The previous cases of taboos are including the taboos temporarily, i.e., until any legal obstacle vanishes. They divide into six types:

A) The woman, who is married from another man, whether she is Muslim or non-Muslim, until her husband divorces her or dies, for the Quranic verse:

Nor can you marry the women who are married except the captives on hand

$$
\text { (Al-Nisa }=\text { The Women, verse 24) }
$$

As well as the divorced woman who spends her course (three months or menstrual cycles), whether the divorce is retroactive or not or even because of the death, for the Quranic verses:

Divorced women shall remain for three months, or menstrual cycles, in order to regard their condition

$($ Al-Baqarah $=$ The Heifer, verse 228)

And in the event of a husband's death, bereaved widows shall wait for a determined period of four months and ten days without any expectation other than the possibility of pregnancy, inevitably shaping 
the course of events. When the determined period of waiting has expired, those of you of influence are absolved of their inclinations as long as they are equitably and honourably disposed, and Allah is Khabirun (Omniscient) of all you do

(Al-Baqarah $=$ The Heifer, verse 234)

As for those who are in process of gestation, their set period accords with the duration of the pregnancy and ends when they have been relieved of their burden; and he who entertains the profound reverence dutiful Allah, will Allah facilitate his task and lead him to a facile and useful life

$$
(\text { Al-Talaq }=\text { The Divorce }, \text { verse } 4)
$$

B) The wife's sister, daughter, mother or grandmother, paternal and maternal aunts, and her niece on the brother and sister's side, until she is divorced or her husband dies, for the Quranic verse:

Nor can you effect a, matrimonial union with two sisters at the same time

(Al-Nisa $=$ The Women, verse 23).

C) The woman who has been divorced three times form one spouse until she marries from another and separates from him for a legitimate reason such as the divorce or the death, for the Quranic verse:

Marriage is not a chess game where a man can move his wife as he pleases from one square to another on a board. Marriage is an article of faith secured by religious sentiment, reverence and a sense of justice against violation. Among the legislative provisions consented to by Allah divorce is «the most objectionable to Him.» The prophet said that a man who treats it lightly must pay the price, sometimes humiliation. If he happens to divorce her and reclaim her twice and then divorces her again a third time, then he has no right to reclaim her in wedlock, that is if they both are so inclined, before joining another man 
in wedlock. If the second husband willingly divorces her, then and only then, are they absolved to reunite in wedlock if they both believe they are able to honour the limitations imposed by Allah. These are the limitations imposed by Allah; He clearly explicates them to those who reflect

(Al-Baqarah $=$ The Heifer, verse 230)

D) The woman who is imprecated by her husband until he retracts his claim. This is expressed by the Quranic verses:

And when those who accuse their wives of adultery have no witnesses but themselves, then the evidence given by any of them alone shall be accepted if he binds himself by an oath solemnly declared four times in attestation of the truth of his statement. Then the fifth object to which he is sworn is that of an invocatory prayer inviting Allah's curse upon himself if he were lying. And the expedient which would avert her -the wife's- punishment is that she binds herself by an oath solemnly declared four times in attestation of the falsity of his - her husband's - statement. And the fifth object to which she is sworn is that of an invocatory prayer inviting the wrath of Allah upon herself if he were telling the truth

$($ Al-Nur $=$ Physical and Spiritual, verses 6, 7, 8, 9)

E) The woman who does not embrace a divine religion until she does, for the Quranic verse:

Do not join idolaters in wedlock before they believe in Allah, their Creator, with true faith. Indeed, a slave who believes in Allah is far better than an idolater even if adorned with qualities attracting your love; nor should you allow your women to join idolaters in wedlock before believing in Allah with spiritual faith; indeed, a slave who believes in Allah is far better than an idolater even if adorned with qualities attracting your love. These idolaters influence your actions and 
deeds and you will end in the Fire, whereas Allah graciously attracts you to settle in His Paradise, and enjoy His forgiveness. Allah explicates to people His revelations that they may hopefully open their minds' eyes

$($ Al-Baqarah $=$ The Heifer, verse 221)

F) The fifth wife if the man is married from four wives until he leaves one of them by divorce or death.

30. The Method of Agreement states that the cause and effect are inseparable in the occurrence, so that if the first occurs, the second will follow it. As for the Method of Difference, it states that if we observe several facts that prove that (a) is the cause of (b), these observations are not evidence that (a) is the cause of (b), but we must prove that there is no cause of the event (b) but the event (a). This requires that we must perform negative experiences, i.e., we must use the Method of Elimination, in order to make sure that whenever (a) is absent, (b) is absent also. It is noticeable that the two methods are the same as the two latter conditions of cause in the Islamic qiyas.

31. The Axiomatic is a critical movement that studies the fundamentals and principles of the proof in a specify science, especially the Geometry.

See: Muhammad Thabit al-Findi, Philosophy of Mathematics (Falsafat al-Riyyadah), The House of University Knowledge (Dar al-Ma'rifah al-Jami'iyyah), Alexandria, 1990, pp. $67 \mathrm{FF}$.

32. For more details about the deductive system and its conditions of construction, see Salah Osman, Scientific Model between Reality and Imagination (Al-Namuzaj alIlmi bayn al-Khayal wa al-Waqi'), Establishment of Knowledge (Munsha't al-Ma'arif), Alexandria, Egypt, 2001, Ch. 5, pp. $87-100$.

33. Al-Ghazali, Al-Mustasfa, Op. Cit., Vol. 2, p. 288, also Abd al-Karim Zidan, Summary of the Fundamentals of Jurisprudence, Op. Cit, pp. $212-213$. 
34. Al-Ghazali, Op. Cit., Vol. 2, p. 289.

35. Ibid, p. 296.

36. Abd al-Karim Zidan, Op. Cit., pp. $214-215$.

37. Mahmoud Fahmi Zeidan, On the Philosophy of Language, (Fi Falsafat al-Lughah), The House of Arab Raising (Dar al-Nahda al-Arabiyyah), Beirut, 1985, pp. $99-100$.

38. Abd al-Karim Zidan, Op. Cit., pp. $209-210$.

39. Ahmad Mahmoud Subhi, This is My Book, Read it, Op. Cit., pp. 160 - 61, also Ali Sami Al-Nashar, Methodology of the Islamic Thinkers and the Discovery of the Scientific Method in the Islamic World, Op. Cit., p. 120.

40. Ali Sami Al-Nashar, Op. Cit., p. 124.

41. Abd al-Karim Zidan, Op. Cit., pp. 217 - 218.

42. Ibid, pp. 218 - 19, also Hassan Abdel Hamid \& Muhammad Mahran, On the Philosophy of Science and Methodology, Op. Cit., pp. $71-73$.

43. Abu Hamid al-Ghazali, Logic of Incoherence of the Philosophers (Mantiq Tahafut al-Falasifa al-Musama M'iyar al-Ilm), ed. by. Sulyman Dunya, The House of Knowledge (Dar al-Ma'arif). Cairo, 1961, p. 176.

44. Al-Shirazi, The Bright Light in the Fundamentals of Jurisprudence, Op. Cit., Vol. 1, p. 2.

45. Ahmad Amin, The Forenoon of Islam: the emergence of Science in the First Abbasid Age (Duha al-Islam: Nash'at al-Ilum fi al-Asr al-Abasi al-Awal), The General Egyptian Book Organization (Al-Hay'ah al-Misriyah al-A'ma li al-Kitab), Cairo, 1998, pp.166 - 67.

46. Quoted from Nasir Ibn Sulyman al-Omar, The Difference in Islamic Action: Causes and Effects (Al-Ikhtilaf fi al-Amal al-Islami: al-Asbab wa al-Nata'ij), a paper presented to the scientific conference entitled: Islamic Action between Agreement and Disagreement, University of Khartoum, Department of Islamic Culture, 12 - 14 July 2004, p. 4.

47. Ahmad Mahmoud Subhi, This is My Book, Read it, Op. Cit., pp. $162-63$. 
48. Zaki al-Din Sh'aban, Fundamentals of Islamic jurisprudence (Usul al-Fiqh al-Islami), quoted from Ahmad Mahmoud Subhi, Op. Cit., p. 164.

49. See Ahmed Amin, Op. Cit., pp. 157 FF, also Ahmad Mahmoud Subhi, Op. Cit., pp. 168 - 69.

50. These schools (madhahib) according to their founders' names are:

- Al-Hasan al-Basri (642 - 728)

- Abu Hanifa al-N'uman (699 - 767)

- Abd al-Rahman al-Awzai (707 - 774)

- Sufyan al-Thawri (d. 778)

- Al-Layth Ibn Sa'ad (716 - 791)

- Sufyan Ibn Uyaynah (725 - 813)

- Muhammad Ibn Idris al-Shafi'I (767 - 820)

- Ishaq Ibn Rahawyah (779 - 852)

- Abu Thawr (764 - 854)

- Ahmad Ibn Hanbal (780 - 855)

- Dawũd al-Zahiri (d. 882)

- Ibn Jurayr al-Tabari (838 - 923)

- Malik Ibn Anas (715 - 796) In addition to the school of:

- Ja'far al-Sadiq (703 - 765) in Shi'a Imams

- Zayd Ibn Ali (698 - 740) in Zaidiyyah, from which the school of Imam al-Hadi ila al-Haq Yahya Ibn al-Hassan al-Qasim al-Rassi branched,

- Jabir Ibn Zaid al-Azdi in al-Khawarij

51. Ahmed Amin, Op. Cit., pp. 173 - 74.

52. Ahmad Mahmoud Subhi, Op. Cit., p. 172.

53. Ibid, pp. $175-76$.

54. This view is based on the following story, which is circulated among many of the historians of Kalam's schools: "Someone came into a meeting of Al-Hasan alBasri $(642$ - 728) and said: 'O Imam al-Din (leader of religion), a group has emerged in our time to declare that the perpetrator of a major sin [Murtakib al-kabirah] is a 
disbeliever. The major sin, according them, is tantamount to blasphemy that leads to departures from the faith. Those are the Kharijets. Another group postpones the decision on the perpetrator of guilt until the Day of Judgment. They say that the major sin is not conflicting with the faith, because the deed is not a pillar of faith; the disobedience does not harm one if he is believer, and the obedience is not useful one if he is an infidel. Those are the Murjites (who say that only God can judge who is a true Muslim and who is not, and no one else can judge another as an infidel). What is your decision in this matter? Al-Hassan took some time to think about the answer, but before he answers, Wasil Ibn At'a (700 - 748) said: 'I am not saying that the perpetrator of a major sin is a believer absolutely or a disbeliever absolutely, but he is in an intermediate position between the two; he is not a believer, nor a disbeliever'. Then, Wasil got up and withdrawed to a column of the mosque in order to confirm his answer among some of al-Hassan friends, and al-Hassan said: 'Wasil withdrawed from us'. Since that time, Wasil and his friend were called 'Mu'tazilah'».

See Ahmad Mahmoud Subhi, 'On the Science of Kalam: a Philosophical Study of the Opinions of Islamic sects in the Origins of Religion' (Fi ilm al-Kalam: dirassah falsafiyah li ar'a al-Firq al-Islamiyah fi usul al-Din), Op. Cit, Vol. 1: Al-Mu'atazilah, pp. $101-102$ \& pp. $184-185$.

55. See Muhammad Shams al-Din al-Dhahabi, 'The Book of the Major Sins' (Kitab al-Kaba'ir), The Scientific House of Books (Dar al-Kutub al-Ilmiyyah), Beirut, 2000.

56. The Basic Statement of faith becoming a Muslim is 'I testify that none has the right to be worshipped but Allah Alone, and Muhammad is the Messenger of Allah (= $\mathrm{La}$ ilaha il Allah, Muhammad Rasool Allah)'.

57. Ahmad Mahmoud Subhi, Op. Cit., p. 162.

58. Ibid, pp. $141-42$.

59. Ibid, pp. $152-54$.

60. Ibid, p. 285. 
61. Ibid, p. $290-293$.

62. Ibid, pp. 330 - 332, also Ali Sami Al-Nashar, Methodology of the Islamic Thinkers and the Discovery of the Scientific Method in the Islamic World, Op. Cit, p. 146.

63. Abu Ja'far al-Tahawi, 'The Explanation of al-Tahawiyyah according to the Salafi Creed' (Sharh al-Tahawiyyah Ala al-Aqidah al-Salafiyyah), quoted from Ahmad Mahmoud Subhi, Op. Cit., p. 342.

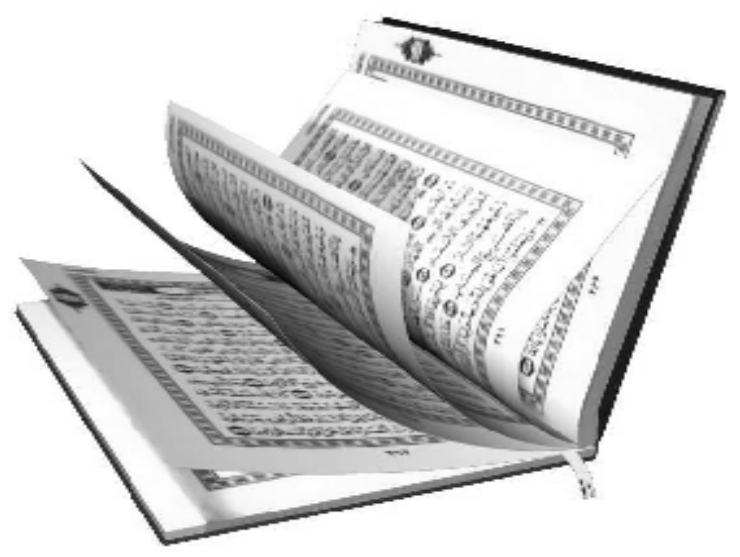




\section{A Dialogue between Authors: Neutrosophy and Arabic Philosophy}

(in conclusion)

FS: Neutrosophy is alike metaphilosophy, I mean a philosophy of the philosophy (= study, interpretation, re-interpretation, hermeneutics of the whole philosophy).

«Neutrosophy, a new branch of philosophy» is organized in a systemized way, which comprises: etymology, definition, characteristics, methods of neutrosophic study, formalization, main principle, fundamental thesis, main laws, mottos, fundamental theory, delimitations from other philosophical concepts and theories, etc.

SO: Since the Arabic culture is an important part of the human culture, we re-interpret the Arabic philosophy from a neutrosophic point of view.

FS: There are ideas/propositions $<\mathrm{A}>$ such that both $<\mathrm{A}>$ and $<$ antiA $>$ are simultaneously true. For example, if $<\mathrm{A}>$ $=$ 'Movie $\mathrm{X}$ is good', then $<\operatorname{antiA}>=$ 'Movie $\mathrm{X}$ is not good'; and it is possible that both propositions be true in the same time, i.e. movie $\mathrm{X}$ is good since actor John plays very well, but movie $\mathrm{X}$ is bad since actor George plays very bad; (or you may choose other attributes to judge a movie, or an idea, a proposition).

Similarly, dialetheism, in modern logics, asserts that some contradictions are true.

SO: Neutrosophy supposes that any human idea, thought, system, etc. is not complete. 
FS: Yes, we can say that, since for any idea $<A>$, a counteridea $<$ antiA $>$ and a neutral/ignorant idea $<$ neutA $>$ are generated.

SO: The human mind is incompetent, so the neutrosophic study asserts as well that we may need a God, a Messenger, a Heavenly book, or generally a religion. (This aim finds a great admiration from both Arabic and Islamic worlds, and from any religious man in the Western world) .

Under the misunderstanding between the Western world and the Arabic - Islamic one, and because there is a big gap between both worlds' thoughts, we wished to present a clear and right picture - to the Western reader - about Arabic philosophy and culture.

We reciprocally desire to acquainting the Arabic reader what are the most modern directions in philosophy and logic (Neutrosophy and Neutrosophic Logic at the moment, for example).

FS: Neutrosophic Logic is a generalization of the fuzzy logic (especially of intuitionistic fuzzy logic). It is used not only in philosophy, but has technical and scientific applications too.

SO: I sought for our Arabic and Islamic books, what objects and ideas can support the theory of neutrosophy, and I found a lot of them. There are many of Arabic (Islamic) thinkers, theories, and dogmas, which I used as references, and quoted the examples from them; for example: Mysticism , Monotheism , Fatalism , Averrhoism , Avicennism, Ilm al-Kalam (Muslim scholastic philosophy), The jurisprudence, etc. 
Moreover, there are some books about the Pharaonic thought (the ancient Egyptian thought), and the modern Arabic thought.

FS: Look at these examples of Neutrosophy used in Arabic philosophy:

- While Avicenna promotes the idea that the world is contingent if it is necessitated by its causes, Averroes rejects it, and both of them are right from their point of view.

Hence $<\mathrm{A}>$ and $<$ antiA $>$ have common parts.

- Islamic dialectical theology (kalam) promoting creationism was connected by Avicenna in an extraordinary way with the opposite AristotelianNeoplatonic tradition.

Actually a lot of work by Avicenna falls into the frame of neutrosophy.

- Averroes's religious judges (qadis) can be connected with atheists' believes.

- al-Farabi's metaphysics and general theory of emanation vs. al-Ghazali's Sufi writings and mystical treatises [we may think about a coherence of al-Ghazali's «Incoherence of the Incoherence» book].

- al-Kindi's combination of Quranic doctrines with Greek philosophy.

- Islamic Neoplatonism + Western Neoplatonism. Etc.

We can compare not only Arabic thinkers and thoughts among them, but also Arabic thinkers and thoughts with Western and Eastern (Buddhism) ones.

SO: I'm very happy with your interest in the Arabic thought, and in your plentiful acquaintance with the Arabic culture. That means that our common book becomes richer (God willing!). 
Your examples are worthwhile and meritorious, I use them largely, and add other examples, such as Ibn Khaldun's statements in his theory on the cyclic sequence of civilizations, for example:

- Luxury leads to the raising of civilization (because the people seek for comforts of life) but also Luxury leads to the decay of civilization (because its correlation with the corruption of ethics).

As you showed, both statements are right, hence $<\mathrm{A}>$ and $<$ antiA $>$ have common parts!

- On the other hand, there is the inferring of the unknown from the existing known (qiyas al-Gha'ib ala al-Shahid) in Islamic jurisprudence, in which we find the same principles and laws of neutrosophy.

- In fact, we can also function a lot of Arabic aphorisms, maxims, Quranic verses (Ayat alQur'ãn) and Sunna of the prophet, to support the theory of neutrosophy.

Take the colloquial proverb that «The continuance of state is impossible» too, or «Everything, if it's increased over its extreme, it will turn over to its opposite»!

I just agree with you that it is important to compare the Arabic thinkers and thoughts with Western and Eastern ones, because any theory or dogma in the Arabic world, has been influenced by the foreign theories and dogmas, and vice versa.

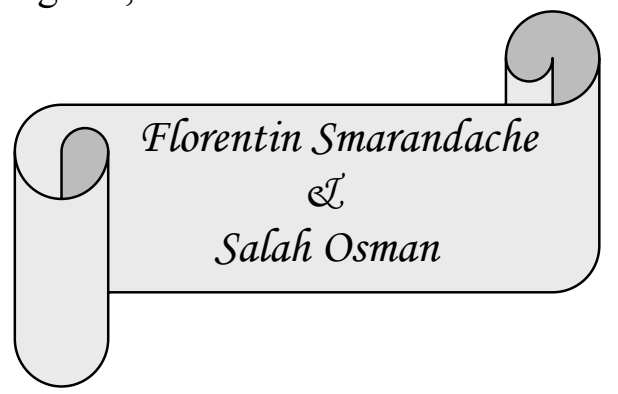




\section{Name Index}

Abduh, Muhammad (1849 - 1905), 108

Ab'lard, P. (1079 - 1142), 32

Abu Bakr (al-Siddiq) (573 - 634), 159,160

Abu-Hanifa (696 - 767), 84

Abu Thawr (764 - 854), 233

Al-Ash'ari, Abu al-Hasan (d. 935), 134

Al-Askari, Muhammad al-Mahdi Ibn al-Hasan (b. 868), 159

Al-Awzai, Abd al-Rahman (707 774), 233

Al-Basri, Al-Hasan (642 - 728), 233, 234

Al-Biruni, Abu Rihan (973 - 1048), 126

Al-Bitruji (Alpetrogius) (d. 1204), 126

Alexander the Great (356 BC-323 BC), 75

Al-Farabi, Abu Nasr (Alpharabius) $(950$ - 872), 88, 122, 126, 139, 140, 143

Al-Farahidi, Al-Khalĩl Ibn Ahmad $(718-767), 84$

Al-Ghazali, Abu Hamid (1058 1111), 123, 124, 125, 134, 143, 161, 162, 164, 174, 194, 205, 224, 225, 226, 232

Al-Hallaj, Mansur (858 - 922), 133, 134

Ali, Nabil (b. 1938), 10, 13

Al-Iji, Adud al-Din (1281 - 1355),
132

Al-Jahni, Ma'bid Ibn Khalid, 120

Al-Juba'i, Abu Ali (d. 915), 216

Al-Juba'I, Abu Hashim (d. 933), 216, 217, 218

Al-Juwayni, Imam al-Haramayn $(1028$ - 1185), 172, 224

Al-Khayyam, Omar (1044 - 1123), 126

Al-Khayyat, Abu al-Husayn (d. 951), 211, 212

Al-Kindi (Alkindus) (801 - 873), 88,126

al-Ma'mun, Abu Jafar (833 - 786), $111,113,163$

Al-Maturidi, Abu Mansur (d. 944), 134

Al-Mawardi, Abu al-Hasan (d. 1058), 140

Al-Mu'tadad, Abu al-Abas (857 902), 161

Al-Mutawakkil (d. 861), 133

Al-Muwaffaq (d. 891), 133

Al-Nashar, Ali Sami, 82, 121, 149, $154,161,162,227,232,235$

Al-Nazzam, Ibrahim Ibn Sayyar (d. 840), 138

Al-Qadir (d. 1031), 133

Al-Qinai, Matta Ibn Yunus (d. 940), 142, 143

Al-Razi, Abu Bakr (Rhazes or Rasis) (865 - 952), 154, 226

Al-Razi, Fakhr al-Din (1149 1209), 144 
Al-Sadiq, Ja'far (702 - 765), 136, 233

Al-Sadiq, Ismail Ibn Ja'far (721 755), 159

Al-Shafi'i, Muhammad Ibn Idris (767 - 820), 93, 204, 224, 225, 233

Al-Shatibi, Ibrahim Ibn Musa Ibn Muhammad (d. 1288), 100, 156, 198

Al-Shawkani, Muhammad Ibn Ali (1760 - 1834), 174, 181, 205, 225,227

Al-Shirazi, Qutb al-Din (1236 1311), 126

Al-Sirafi, Abu Sa'id (d. 978), 142

Al-Tabari, Ibn Jurayr (838 - 923), 233

Al-Tahawi, Abu Ja'far (d. 933), 134, 218, 235

Al-Tawhidi, Abu Hayyan (930 1023), 142, 165

Al-Thawri, Sufyan (d. 778), 233

Al-Tilmisani, Afif al-Din (d. 1291), 128

Al-Tusi, Nasir al-Din (1201 1074), 126

Al-Wathiq (d. 847), 133

Al-Zahiri, Abu Dawud (d. 882), $165,204,233$

Al-Zahiri, Ibn Hazm (994 - 1069), 165

Al-Zamakhshari, Abu al-Qasim (1074-1143), 144

Aristotle (384 BC - 322 BC), 125, 141, 143, 194, 207, 208

Atanassov, K. T. (b. 1954), 52, 63

Bacon, F. (1561 - 1626), 43, 44, 183

Bartold, Vasily Vladimiroyich (1869 - 1930), 74, 83, 145, 149 , Bayes, Reverend Thomas (1702 -
1761), 51

Bergson, Henri (1859-1941),42,129

Bradley, F. H. (1836 - 1924), 30

Bohr, Niels (1885 - 1962), 31, 60

Bool, G. (1815 - 1864), 48

Brown, E. G. (1862 - 1929), 81, 82, 149

Bush, George (b. 1946), 150

Cantor, G. (1845 - 1918), 37

Carlyle, T. (1795 - 1881), 106

Carnap, R. (1891 - 1971), 44, 61

Copernicus (1473 - 1543), 86

Corbin, Henri (1903 - 1978), 81

D'Ailly, Pierre (Petrus de Alliaco) (1350 - 1420), 54

Darwin, Ch. R. (1809 - 1882), 151

Dawn, J. L. (1828 - 1896), 152

De Broglie, L. (1892 - 1987), 59

Dempster, Arthur P., 51,

Derrida, Jacques (1930 - 2004), 44

Dezert, Jean (b. 1962), 8, 67

Diderot, D. (1731 - 1784), 61

Dirac, P. (1902 - 1984), 60

Dubois, Didier (b. 1952), 67

Dummett, Sir Michael Anthony (b. 1925), 52

Dunn, J. Micheal, 49

Einstein, A. (1879 - 1955), 31, 53, 59,66

Emerson, Ralph Waldo (1803 1882), 33

Eminescu, Mihai (Mi'haj) (1850 1889), 31

Engels, F. (1788 - 1839), 22

Eubulides of Miletus (b. 4th century BC), 39

Euclid of Alexandria (325 BC - 265 BC), 126

Fine, Kit (b. 1946), 52 
Frege, Gottlob (1848 - 1925), 143, 165

Fresnel, A. (1788 - 1827), 59

Galileo Galilei (1564 - 1642), 86

Galton, F. (1822 - 1911), 150

Goguen, Joseph (b. 194), 50

Goldzher, I. (1850 - 1921), 81, 83

Good, Irving John (or Jack) (b. 1916), 51

Gottschalk, H. (b. 1919), 78

Grelling, Kurt (1886 - 1942), 55

Hegel, G. W. F. (1770 - 1831), 3, $8,18,21,22$

Heraclitus (576 BC $-480 \mathrm{BC}), 2$, $13,31,45,110,117$

Hertz, H. (1857 - 1894), 59

Huygens, C. (1629 - 1695), 59

Ibn Abd al-Aziz, Omar (680 - 720), 209

Ibn Abi Sufyan, Muawiya (602 680), 160

Ibn Abi Talib, Ali (599 - 661), 119, $158,159,160$

Ibn Abi Talib, Jafar, 95

Ibn Abi Ubayd, Al-Mukhtar (622 687), 119

Ibn Abi Waqqas, Sa'd, 101

Ibn Affan, Uthman (574 - 656), 119, 206

Ibn Al-Awam, Al-Zubayr (593 656), 119

Ibn al-Azraq, Nafi, 160

Ibn Al-Farid, Umar (1182 - 1235), 128

Ibn Al-Hanafiya, Muhammad (637 $-710), 161$

Ibn al-Hassan, Yahya, 233

Ibn Al-Haytham, Al-Hasan (965 1039), 126

Ibn Al-Hussuyn, Zayd Ibn Ali (698
$-740), 159,233$

Ibn Al-Jawzi, Abu al-Faraj (1126 1200), 128

Ibn A'mir al-Hanafi, Najda, 160

Ibn Anas, Malik (715 - 796), 136, 204, 233

Ibn Arabi, Muhyiddin (1165 1241), 128

Ibn Al-Asfar, Ziyad, 160

Ibn At'a, Wasil (700 - 748), 234

Ibn Bajja, Abu Bakr Muhammad (Avenpace) (1095 - 1138), 122

Ibn Hanbal, Ahmad (780 - 855), 131, 133, 136, 203, 204, 206, 233 Ibn Ibadh Al-Tamimi, Abd Allah, 160

Ibn Ishaq, Hunayn (Johannitius) (808-873), 113, 114

Ibn Khaldun, Abd al-Rahman (1332 - 1406), 79, 80, 130, 140, 148

Ibn Luqa, Qusta (820 - 912), 114

Ibn Mas'ud, Abd Allah, 101, 102

Ibn Miskawayh, Abu Ali Ibn Ya'qub (932 - 1030), 69

Ibn Muawiyya, Khalid Ibn Yazid $(635-704), 111$

Ibn Qurra, Thabit (826 - 901), 114, 126

Ibn Rahawyah, Ishaq (779 - 852), 233

Ibn-Rushd, Abu al-Walid (Averroes) (1126 - 1198), 88, 126

Ibn Sab'a, Abd Allah, 159

Ibn Sa'ad, Al-Layth (716 - 791), 233

Ibn Sina, Abu Ali al-Husayn (Avicenna) (980 - 1058), 88, 122, 126, 162, 165,

Ibn Taymiya, Abu Abas (1263 1328), 128, 129

Ibn Thabit, Khuzaymah, 176, 177, 226 
Ibn-Tufail, Abu Bakr Muhammad (Abubacer) (1105 - 1185), 122

Ibn Ubayed Allah, Talha (596 656), 119

Ibn Uyaynah, Sufyan (725 - 813), 233

Ibn Zaid al-Azdi, Jabir, 233

James, William (1842 - 1910), 34 Justinian, Flavius Anicius (483 595), 76

Kemal, Mustafa (Atatũrk) (1881 1938), 147

Keynes, John Maynard (1883 1946), 29

Kleene, Stephen Cole (1909 1994), 49

Klein, Felix Christian (1849 1925), 52, 53, 66

Koopman, Bernard O. (1900 1981), 51

Lambert, J. H. (1728 - 1777), 51

Le Bon, Gustave (1841 - 1931), 79, 148

Leibniz, G. W. (1646 - 1716), 19, 47, 53

Lenard, Ph. (1862 - 1947), 59

Lewis, J. (1941 - 2001), 153

Lukasiewicz, Jan (1878 - 1956), 49

Mahmoud, Zaki Naguib (1905 1993), 108, 154, 157, 162

Marx, Karl (1818 - 1883), 20, 21, 22

Maxwell, J. C. (1831 - 1879), 59, 60

Mendeleev, D. I. (1834 - 1907), 30

Mill, John Stuart (1806 - 1873), 183

Muhammad, The Prophet (PBUH), 70, 79, 92, 99, 116, 153, 156,
$158,159,161,179,185,189$, 213, 219, 223, 234

Moore, G. E. (1873 - 1958), 188

227, 232, 233, 234, 235

Narinyani, Alexandre, 52

Negus, King of Abyssinia, 95

Newton, Isaac (1643 - 1727), 25 , $26,59,70$

Nietzsche, F. (1844 - 1900), 42, 44, 152

Osman, Salah (b. 1963), 1, 12, 67, $143,151,157,220,231$

Pasch, M. (1843 - 1930), 183

Peirce, Charles S. (1839 - 1914), 48,66

Plato (428 BC - 348 BC), 13, 215

Plekhanov, Georgi Valentinovich (1856 - 1918), 32

Post, Emil Leon (1897 - 1954), 48, 49,

Prade, Henri (b. 1953), 67

Qarmat, Hamdan (890 - 906), 161

Quine, W. Van O. (1908 - 2000), 48

Reichenbach, Hans (1891 - 1953), 49

Rousseau, J. (1712 - 1778), 43, 45

Ruginã, Anghel N., 29

Sabra, A. I., 87, 145, 154, 158, 161, $162,163,164,165$

Schopenhaur, Arthur (1788 - 1860), 43

Schrödinger, E. (1887 - 1961), 60

Shafer, Glenn, 51, 52,

Sibawayh, Abu Bishr Amr Ibn Uthman (750 - 795), 83, 149,

Smarandache, Florentin (b. 1954), 4 , $5,7,8,9,12,13,20,41,67,69$, 
70,148

Subhi, Ahmad Mahmoud, 145, 148, $153,156,157,158,163,164$

Socrates (Sǒcratēs) (470 BC - 399 BC), 22, 40, 43

Spengler, O. (1880 - 1936), 75, 145

Spinoza, Baruch (1632 - 1677), 31

Steuco, Agostino (1496 - 1549), 19
Van Fraassen, Bas C. (b. 1941), 52

Voltaire, F. (1694-1778), 51

Wahl, J. Andrẻ (1888 - 1974), 30

Walras, Leon (1834 - 1910), 30

Whitehead, A. N. (1861 - 1947), 42

Whitman, W. (1819-1892), 26

Wittgenstein, L. (1889 - 1951), 28

Turgenev, Ivan Sergeyevich (1818 Yager, Ronald, 67

$-1883), 24$

Toynbee, A. (1889 - 1975), 75, Zadeh, Lotfi Asker (b. 1921), 40, 152 50,67

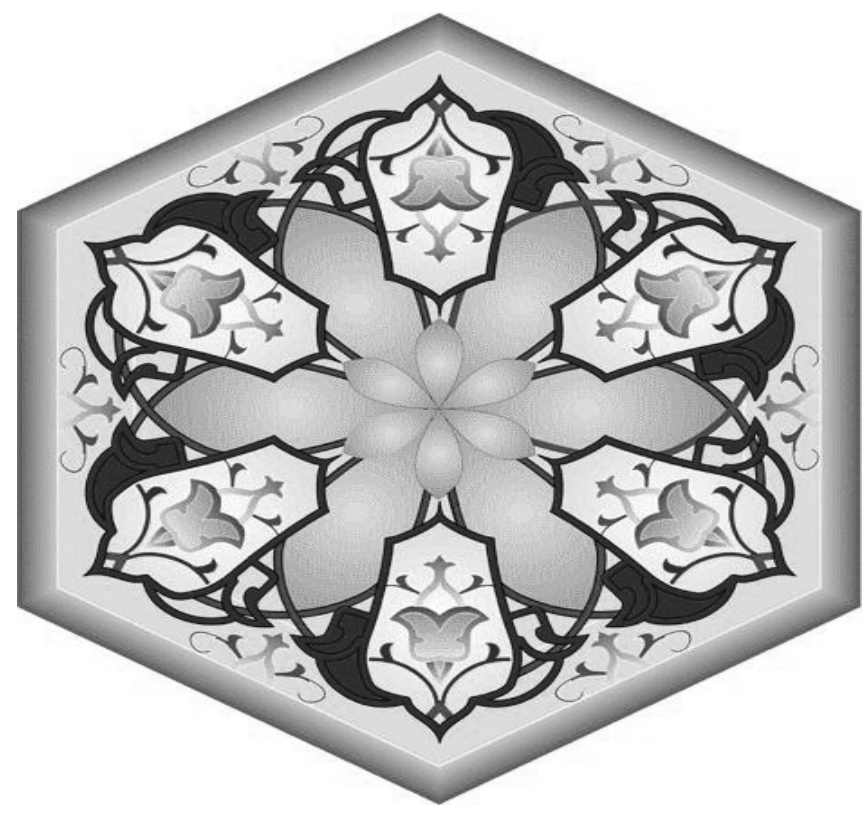




\section{Subject Index}

Abbasid age, 111, 232

Absolute, 23, 24, 26, 28, 35, 47, 48, 53, 54, 56, 62, 74, 91, 101, 131, 199, 205, 207, 210, 212,

absolute indeterminacy, 54 absolute truth, 26, 35, 47, 48, 53, $54,56,62,91,199$

Absoluteness (Tanzih), 118, 210, 211,214

Abyssinia (Habesha), 95, 96

Action, 2, 11, 37, 70, 77, 97, 98, $102,105,131,155,156,200$, $210,211,214,223,231,232$

Admiration (Istihsan), 138, 201

Advaita, 34

Al-Asha'irah (Ash'arites), 164, 213, 214, 215

Al-Azraqa, 160

Algebraic, 33

Algorithm (s), 8, 67

Al-Ibadiyyah, 160

Al-Jahmiyyah, 163

Al-Kaysani Shi'a, 119

Al-Khawarij (Kharijites), 119, 133,

138, 160, 209, 210, 212, 233

Al-Maturidiyyah, 134

Al-Murji'a or Murjites (The postponers), 209, 210, 234

Al-Mu'tazilah (Mu'tazilets), 160,

161, 163, 164, $208-11,213,234$

Al-Qadiriyyah, 163

Al-Qaramita, 119

Al-Sufiryyah, 160

Al-Tahawiyyah, 134, 235
Al-Zahiriyah, 138, 164, 204

Al-Zaidiyya (Fivers), 119, 159

Analogy, 16, 167, 168, 185, 186, 191, 194

Analysandum, 187, 188

Analysans, 187, 188

Anthropomorphism (Tashbih), 118, 131, 210, 214

Anthropomorphists (Mushabiha), 133, 213, 214

Antimatter, 8

Antioch, 122

Antonym, 16, 217

Aphorism, 15, 80

Appearance, 11, 73, 77, 78, 96, 106, $127,128,159,164$

A posteriori, 19

A priori, 19

Arabian Peninsula, 79

Arabic Language, 73, 74, 76, 81, $82,83,84,85,92,97,134-136$, $141-43,149,154,179,186,191$

Arabs, 71, 73, 76, 78 - 90-97, 98, 106, $109-19,136,141,158$

Argument, 44, 51, 57, 72, 76, 78, $85,87,88,91,124,130,132$, 138, 143, 144, 150, 208, 213

Aristotelianism, 122

Arithmãtĩqĩ, 87

Artificial, 6, 14, 51, 166

Astrology, 88, 112

Astronomy, 87, 88, 112, 115, 206

Atheism (Zandaqa), 133

Atman, 34 
Attributes (Sifãt), 19, 33, 86, 123, $129,140,185,216-18$

Attributists (Al-Sifatiyyah), 217

Autological, 55

Auto-reflexive, 54

Auto-suggestion, 37

Awareness (Ilm), 33, 105, 115, 197, 216-19, 224

Axiom, 41, 46, 54, 183, 231

Axiomatic, 46, 183, 231

axiomatic system, 46

Axiomatization, 30

Badness (Qubh), 210 - 12

Baghdad, 87, 112, 142, 153, 160

Barbaric, 25

Basra, 83, 160

Bedouin, 191, 192, 226

Believer (Mu'min), 70, 108, 118, 164, 178, 179, 200, 209, 210, 222, 223, 234

Benefit, 10, 77, 88, 102, 138, 172, 179, 180, 181, 190, 202, 211

the free (al-Maslih al-Mursalah), $138,172,202$

Bhakti, 34

Biconditional, 65

Bioinformatics, 8

Boundless, 20

Brahman, 34

Branch, 14, 15, 52, 121, 159, 170, $172-79,181,183,192-94$, 203, 233

Buddhism, 34

Cabalistic, 160

Calculus, 49, 58, 154

predicate, 58 neutrosophic p.calculus, 58

Category, 46, 53, 140, 141, 149 sub, 53

Cause, 11, 37, 38, 69, 70, 77, 92, 104, 118, 137, 138, 141, 156,
$167,168,170,172-96,201,211$, 212, 217, 218, 226, 331, 232

Ceaseless anxiety, 38

Central Asia, 87

Certainty, 96, 132, 173, 194

Chance, 46, 51, 52, 178, 214, 222

Chaos, 11, 44, 180

Christianity, 87, 109, 126, 160, 210

Civilization, $72-92,104,107-12$, 141, 145-53, 197, 205

Classical, 8, 14, 16, 29, 45, 48, 50, $51,56,58,166,168$

Clergyman, 146

Color, 16, 24, 60, 64, 152, 181, 191 Complementary colors, 24

Combination, 7, 8, 16, 21, 26, 50, $51,60,67,109,151,166$

Completeness, 17, 70, 95, 184, 218

Conceptualism, 32

Conjunction, 65

Connector, 66

Peirce, 66

Sheffer, 66

Conquest, 75, 91, 92, 103, 109, 110, 114

Conscience, 73, 105, 129, 149, 206, 221

Consistency, 11, 184, 218

Constant, 10, 23, 47, 77, 211

Constantinople, 76, 103

Construction, 4, 22, 54, 82, 92, 231 complement, 54

Context, 41, 43, 54, 72, 74, 76, 127, 141

Continuation (Istisihab), 139, 172

Continuum, 17, 27, 29, 32, 35, 50, 64

power spectrum, 17

Contradiction, 1, 6, 11, 16, 19, 21, $28,55,56,59,66,69,72,141$, $168,169,184,198,207,208,215$

Copula, 213

Corpuscular, 59 
Countable, 53

Custom (Urf), 138, 172

Cybernetics, 67

Dadaism, 24

Damascus, 111, 155, 224, 225

Dense, 30

Denumerable, 35

Desire, 38, 72, 81, 103, 105, 127, 173, 174, 211 inverse, 38

Dialectic, 8, 9, 18, 22, 28, 61, 81, $141,142,160,220$ materialism, 22

Dialects, 141

Dialetheism, 28, 48, 66

Dilemma, 57 constructive, 57 destructive, 57

Disequilibrium, 29, 30, 49, 50 stable, 29

Disjunction, 65 strong or exclusive, 65 weak or inclusive, 65

Disobedience, 133, 199, 201, 209, 225, 234

Distinction, 25, 26, 40, 48, 90, 104, $125,128,143,187,188,196$, 211, 212, 218

Division, 10, 75, 116, 136, 154, 187, 189, 203

Doctrine (Aqida), 3, 79, 83, 84, 86, $88,93,119,120,122,123,124$, $127,128,131,132,134,159$, 160, 161, 163, 164, 204, 206, 216

Dualism, 210, 211

Duality, 16, 34, 50

Dynaphilosophy, 21

Economics, 8, 14, 29, 67, 206

Effect, 11, 24, 35, 58, 59, 92, 148, $155,165,168,183,191,194$, $195,196,227,229,231,232$ photoelectric, 59

Effort (Ijtihad), 89, 103, 123, 132, 135, 170, 204, 206

Ego, 34

Electron, 29, 59, 60,

Emanation, 159, 195

Embodiments (Aqanim), 216

Empty, 19, 22

Enigma, 33

Entailment, 49, 58 classical, 58

Entropy, 2

Epistemology, 18, 28, 215 neutrosophic, 28

Equation, 27, 53, 60 Maxwell's, 60

Equilibrium, 18, 22, 29, 30, 38, 49, 50, 74, 91 unstable, 29

Equivalence, 65, 187, 188 logical, 187, 188

Essence (Dhãt), 33, 43, 104, 120, 129, 144, 145, 168, 186, 191, 208, 216- 18

Eternal, 124, 131, 144, 160, 163, 205, 215, 223

Ethics, 69, 94, 95, 126, 133, 135

Ethnic, 151, 153

Eugenics, 86, 150, 151

Evil, 2, 33, 71, 97, 105, 118, 128, $130-34,138,181,200,202,210$ $-12,219$

Evidence, 30, 51, 69, 75, 99, 129, $130,135,137,138,139,162$, 182, 184, 186, 190, 198, 205, 213, 214, 216, 220, 221, 230, 231 combination of , 51

Existence (Wujud), 41, 45, 60, 78, $104,108,125,132,175,177-83$, 184, 190, 191, 194, 195, 197, $211-19$

Existentialism, 40

Expert, 67, 206 
systems, 67

Fallibilism, 19

False, 19, 26, 28, 31, 35, 41, 42, 44, $47,49,51,52,54-56,59,61-$ $64,66,91,95,100,133,149$, 166, 167, 174, 187, 199, 208, 223

Falsehood (falsity), 1, 9, 10, 47, 48, $49,51,54-56,60,62,66,90$, $91,95,166-69,196,199$

absolute, 54, 56, 199

false, 66

relative, 48,54

super, 55

true, 66

Fast (Sawm), 87, 94, 95, 96, 153, 170, 176, 178, 179, 180, 195, 223

Fatalism, 120

Fate, 112

Father, 216

Fatimids, 119

Fatwa (Legal opinion), 133, 202, 204, 206

Feudalism, 36

Fire-Worshipper (Majus), 85

Folklore, 66

Forbidden (Mahzur), 86, 133, 176, 177, 185, 187, 191, 209, 223, $225-27$

Foreigners (Non-Arabs), 83

Form, 87, 88, 90, 96, 100, 106, 110, $112,125,128,129,143,146$, $160,174,179,183,184,199$, 204, 205, 208, 212, 215, 229 prenex normal, 58, 71

Formation, 83, 85, 119, 189, 197

Formula, 15, 22, 23, 47, 54, 55, 58, $71,117,207$

well-formed, 54, 55, 58

Humanization, 118

Hypothesis, 8, 35, 47, 50, 183

Free benefit (al-Maslaha alMursalah), 138, 172, 202,
Free will, 112, 120, 224

Frequency, 46

Function, 7, 9, 34, 47, 51, 54, 58, 140, 213, 221 belief, 51,

Fundamentalists (Usuliyyun), 98, 99, 170, 180, 183, 188, 189, 191, 192, 194

Fusion, 4, 8, 67, 68, of information, 67

Fuzzy, 2, 7, 14, 40, 47, 50, 51, 52, $62,63,66,67$ logic, 14, 47, 50, 62, 63, 66 sets, 67

Geometry, 7, 52, 66, 231

Gesture (al-Ima'), 184, 186

Goal (Ghayah),

Goodness (Husn), 210 -12

Goths, 76

Grammar (Nahw), 54, 83, 121, $141-43$

Grave sinner (Fasiq), 118

Hadith (The Prophetic traditions), 70, 82, 99, 101, 102, 128, 154, $157,171,172,173,194,198$, 202, 203, 204, 226

Hanabilites, 131, 133, 134, 163

Hanafites, 171, 172, 190

Happiness, 34, 38, 133, 139

Harran, 112

Heap, 3, 52, 64

Hebrew, 148

Hellenism, 111

Heresy (Hartaqa), 99, 146

Hermeneutics, 19, 32, 35

Hermeticism, 112, 126

Herodianism, 77, 145

Heterological, 55

Hinduism, 34

al- Holy, 1, 84, 105, 120, 216, 218

Spirit, 216 
Identity, 73, 77, 82, 90, 104, 109, Jainism, 34 $172,173,188,207,208,216$

Jerusalem, 145

Ignorantism, 31

Ilm al-Kalam (Scholastic theology), 87, 129, 134, 153, 163, 207, 208, 234

Ilm Usul al-Din (The way of life in submission of Allah), 129

Imitation, 69, 132, 137

Implication, 65, 132, 188, 196

Incarnation, 127, 128

Incoherence, 123, 161, 162, 232

Independence, 175, 217

Indeterminacy, 14, 15, 18, 26, 40, $46-51,54-56,60,62$ relative, 48,54 super, 55

Inference, 51, 54, 64, 91, 143, 167, 168,184

Jesus (Al-Masih), 216

Jnãnã, 34

Judaism, 113, 126, 159, 210

Judgment, 2, 38, 80, 91, 93, 94, 97, $99-108,114,115,118,120$, $127,134-39,141,165,167$ 83, $186-98,200,201,202,205$, 208, 209, 212, 215, 234

Jurisprudence (Fiqh), 83, 84, 121, $124,134,136,140,143,154$, $157,162,164,167,203,204$, 205, 207, 224, 226, 227, 232, 233 Jurist, $88,89,98,99,118,127,128$, $129,133-36,138,140,142$, $167,168,170,171,175,176$, 181, 182, 184, 191, 193 - 206, 225

Infidel (Kafir), 111, 124, 150, 159, 160, 164, 199, 209, 210, 234

Infinite, 1, 10, 18, 21, 26, 27, 28, $33,50,59,60,62,70,73,89$, 168, 197, 199, 210

Inseparability, 186, 190, 191

Intelligence, 14, 51 artificial, 14, 51

Interference, 16, 59

Interval, 8, 37, 47, 48, 50, 62, 70

Intrinsicalness, 60

Intuition, 33, 46, 127

Invalid, 79, 133, 135, 180, 189

Invention, 163

Invisible, 39, 40, 116, 150

Ismailis (Ismailism), 119, 159, 161

Ithnã-ashariyya (Twelvers), 119, 158,159

Jaafrites, 138

Jahiliyyah, 79

of identities' disjointedness, 26

of inverse effect, 24

of joined disjointedness, 25

Justice (AdI), 105, 107, 130, 131, $132,164,210-212,220-222$, 229

Divine, 130, 131, 164, 210, 212

Justification，3，18，74，84，105, 120,140

Kashmir, 111

Khwarazm, 112

Kufa, 83

Law, 15, 17, 25, 26, 51, 56, 70, 75, $77,88,94,96,99,109,122,127$, $129,133,136,138,140,147$, $151,156,158,166,167,178$, 197, 202, 206, 207, 208, 215, $216,222,225,227,228$

of anti - reflexivity, 24

of compensation, 26

of complementarity, 24

of contradiction, 56, 169, 184, 207,215

of equilibrium, 22

of excluded middle, 56, 208, 216 
of particular ideational Madhhab, 136

gravitation, 26

of prescribed condition, 26

of reverse identification, 25

Madina, 139, 192

Madianism, 113

Majus, 85

of universal ideational

gravitation, 27

Leader (Imam), 49, 71, 78, 83, 84,

Malikites (Malikitism), 88,136, 204

Mandatory (Muhattam), 137, 170, 221

99, 119, 134, 137, 140, 142, 143, Manicheism, 113, 160

$150,158,161,234$

Manifold, 52

protected leader, 119, 158

Manuscripts, 5

Leadership (Imamah), 140, 159, Maragha, 88 203

Legacies, 73, 154

calculus of, 154

science of, 154

Linguistics, 141

Logic, 3, 7, 8, 11, 13, 15, 18, 28, 45 $-64,66,71,97,112,113,116$, $124,132,135,139,141,142$, $143,144,162,165-69,174,175$, 179, 194, 200, 207, 220, 232

Aristotelian, 143, 167, 169, 207

bivalent, 48,64

Boolean, 48

designing control, 63

fuzzy, 14, 47, 50, 62, 63, 66

infinite-valued, 50

intuitionistic, 28, 58

intuitionistic fuzzy, 47, 63

many-valued, 50, 139, 167, 168, 194, 220

modal, 53, 63

neutrosophic, 3, 7, 8, 13, 14, 16, $28,45,46,47,48,50,51,52,55$, $56,57,58,62,63,64,66,139$, $167,168,169,193,208,212$

non-classical, 45

of illogic, 66

paraconsistent, 28

plurivalent, 49

two-valued, 166

Macro-philosophy, 33

Materialism, 22, 23 dialectic, 22

Mathematization, 16, 23, 30

Matriarchate, 36

Matrix, 71

Measurement, 67,138,167, 169, 197 sensor, 67

Mecca, 78, 153, 157

Mechanics, 14, 49, 59, 63 quantum, 14, 59, 63

Mediocrity, 42, 60

Meritorious (Fadila), 137

Mesopotamia, 112

Metahistory, 7

Meta-language, 54

Meta-philosophy, 33

Method, 15, 16, 49, 56, 67, 90, 91, $92,93,98,99,100,103,104$, $120,122,135,136,140,144$, $170,172,183,187,188,197$, 201, 204, 227, 231, 232, 235 of agreement, 183, 231

of concomitant variation, 183

of difference, 183, 231

of eliminations, 183

of residues, 183

Midman, 42, 43

Modality, 54

Model, 59, 119, 121, 166, 208, 231

Transferable Belief (TBM), 67

Modes or States (Ahwal), 216, 217

Modus Ponens, 56, 174 
Modus Tollens, 56

Mongolism, 152

Monism, 18

Monotheism (al-Tawhid), 93, 95, 130, 131, 132, 210, 216, 217

Motto, 18

Multi-space, 7

Multi-sensors, 67

Multi-structure, 7

Mutakallamun, 130

Mysteries, 41

Mysticism, 87, 94, 121, 126, 127, $128,129,134,162$

NASA, 68

Naturalization, 121, 125, 161

Negation, 1, 7, 9, 10, 11, 22, 24, 35, $28,58,65,71,90,92,172$ double, 58

Negativity, 31

Negroes, 119

Neohegelians, 30

Network, 14, 52, 67 neural, 14, 52, 67

Neutral, 10, 15, 18, 19, 21, 29, 73, 91, 168, 203

Neutrality \& Neutralities, 2, 9, 10 , $15,16,17,18,22,23,28,29,32$, $60,62,64,72,77,80,168$

Neutrino, 29

Neutron, 21, 29

Neutrosophic, 3, 7, 8, 9, 11, 13, 14, $15,16,26,28,29,33,34$, $42-74,89,91,110,139,144$, 166 - 69, 193, 199, 208, 212 philosophy, 14, 15, 42

probability, 8, 13, 14, 16, 29 set, $7,8,13,14,47,59$ tree, 110

Neutrosophy, 3, 8, 9, 10, 12, 14, 15, $18,19,29,31,32,33,35,36,48$, 236, 237, 238

Nihil (Adam), 212, 214, 215, 216 ,
218

Nihilism, 11, 19, 20, 24

Nihility, 11, 96

Nondemocracy, 36

Non-duality, 34

Nonlinear, 27

Nonsense, 18

Nuclei, 29

Oath, 178, 179, 220, 222, 230, Obedience, 106, 128, 133, 199, 234

Obligatory, 135, 137, 153, 169

Observation, 15, 45, 46, 87, 115, 126, 231

Ontology, 215, 216

Operator, 47, 58, 63, 64

Ordeal (Mihna), 2, 131, 133, 134

Organicism, 22

Orientalist, 76, 78, 79, 81, 161

Origin (AsI), 4, 15, 89, 95, 118, 119, $126,134,139,150,153,162$, $164,170-77,181-88,192-$ $94,224,225,227,234$

Overinformative, 19

Overman, 42, 43

Paganism, 95

Pantheism, 127, 128

Paradox, 1, 6, 8, 16, 28, 33, 36-41, $48,49,51,52,55,59,63,64,66$, $67,77,174,175$

heterological, 55

invisible, 39

paradox of, 41

pseudo, 66

semantic, 40

set's, 37

short time living, 40

sorites, 39, 49, 52, 64, 67, 174

uncertainty, 40

unstable, 40

Paradoxism, 5, 6, 7, 66, 67, 68

Particle, 14, 26, 39, 40, 59 
submicroscopic, 59

Patriarchate, 36

Peculiarities, 59

Penance or atonement, 169, 178, 179, 222, 223

Penumbra, 52

Permissible or allowed, 117, 118, 135, 137, 189, 196, 202

Perpetrator, 173, 181, 208, 209, 210, 234

the perpetrator of a major sin (Murtakib al-Kabirah), 209, 210, 234

Philosophia perennis (Permanent philosophy), 19

Philosophism, 20, 125

Phobia, 37

Photon, 59

Pilgrimage (Hajj), 78, 87, 98, 100, $117,153,176,180,201,222$ lesser pilgrimage (Omrah), 180

Platonism, 42, 76 neo, 122, 126, 160

Plausibility, 51

Pluralectic, 29

Plurality (Shirk), 21, 61, 131, 216, 218

Poem, 6, 26

artificial, 6; city, 6; dadaist, 6; disseminations, 6; leftist, 6; natural, 6; olfactory, 6; postmodern, 6; sonorous, 6; surrealist, 6 ; tactile, 6 ; taste, 6 ; visual, 6

Polarization, 132, 133

Polemical, 130

Polysyllogism, 57

Postmodernism, 7

Postponement (Irjaa), 118

Pragmatism, 34

Prayer (Salat), 34, 87, 94, 95, 100, $137,147,153,170,171,172$, $176,177,180,181,182,209,230$
Predicate, 52, 58, 59, 217, 218 vague, 52

Predication, 54, 213, 214, 218

Preferable (Mustahab), 84, 137

Prefix, 71

Prescribed (Fard), 26, 137

Prevention of instruments (Sadd alDhrai'i), 138

Probability, 8, 13, 14, 16, 29, 46, 49, 50, 51, 194, 195, 208

objective, 46

Programming, 14

Promise, 117, 122, 130, 131, 201

Prophecy, 88, 99, 117

Prophet's Companions, 97, 138

Proposition, 16, 17, 18, 19, 28, 29, $31,46,47,51-66,127,140,166$ $-69,207,208,213,215,218$ auto-reflexive, 54 categorical, 213

Proton, 29

Providence, 70, 88, 146, 156, 223

Pseudo-morphosis, 77, 145

Punishment, 97, 111, 120, 131, 170, $182,200,201,212,223,224,230$

Qiyas, 138, 166 - 79, 189, 192, 193 $-96,198,200,201,231$

of Equality, 193 of Minimum, 194 of Priority, 193

Quantification, 54

Quantifiers, 71

Quarks, 8

Race, 24, 85, 86, 118，120，129, $151,152,153$

Nordic, 152

Rationalism, 213

Realism, 213

Reason, 30, 37, 70, 77, 78, 89, 90, 98, 99, 102, 104, 105, 107, 113, $118,120,127,128,130,131$, 
$132,135,142,152,163,166,167$, 184, 186, 188, 191, 192, 196, 198, 201, 204, 206, 212, 225, 229 editing (Tahqiq al-Manat), 192 extracting (Takhrij al-Manat), 192

refining (Tanqih al-Manat), 186, 191

Recommended, 131, 135, 137

Reductio ad absurdum, 56

Redundancy, 45, 46

Regularity, 186, 190, 191

Reinterpretation, 16

Relativism, 35

Relativity, 8, 10, 35, 203

Religion, 23, 69, 70, 77, 82, 84, 85, $86,87,89,91,92,95,96,103$, $104,107,111,112,113,120$, $123,126,128,129,130,133$, $135,136,146,151,153,156$, $158,159,160,165,167,170$, 197, 198, 202, 205, 207, 210, 216, 230, 234

Renaissance, 85, 87, 89, 110, 115

Reprehensible or not recommended, 128, 134, 135, 137, 197

Retrogression, 190, 191

Revelation, 10, 88, 91, 92, 97, 98, $102,103,127,135,173,179$, 198,231

Reward, 97, 120, 131, 137, 211

Robot, 8, 67

Sãbians, 85, 112

Samarkand, 88, 134

Saturation, 184

Scholastic, 87, 110, 129

Scholasticism, 87

School, 5, 14, 20, 22, 31, 76, 83, $112,114,121,130,134,136$, 140, 160, 203, 204, 209, 211, 233, 234

monastic, 112

Sect, 103, 119, 121, 123, 132, 133,
$136,144,145,153,158,159$, $160,161,163,165,204,209$, $212,213,234$

Secular, 88, 112, 124, 146, 168

Secularism, 77, 146

Semantics, 48, 49, 52, 135, 144

Semiempiric, 30

Semiscientific, 30

Sensors, 67

Sensualism or Sensationalism, 213

Sequence, 7, 9, 159

Sexuality, 36

Shafi'ites, 171

Shariah (Islamic law), 77, 92, 127, $134,136,138$

Shi'a, 158, 159, 161, 233

Simplicity, 11, 48

Skepticism, 141, 159

Slavery, 36

Software, 5

Somethingness, 212, 214, 215, 216, 218

Son, 5, 102, 158, 211, 216, 227, 228

Space, 7, 10, 23, 43, 54, 77, 85, 90, 91 heterogeneous, 7

Spain, $68,75,88$

Stochasticity, 46

Strange, 122, 141

Subjectivity, 54

Subsets, 48, 50, 62 non-standard subsets, 47, 48, 50, 62

Suitable, 181, 182, 186, 187, 188, 189, 190, 191

free (Al-Munasib al-Mursal), 190 influential (Al-Munasib alMua'thir), 189

proper (Al-Munasib al-Mula'im), 190

Suitability (Munasabah), 175, 182, 188, 189

Sunnah, 91, 92, 98, 99, 100, 102, 
$126,127,129,133,135,137$, pro, 22

$138,139,154,155,165,167$, Threat, 86, 123, 130, 131, 201, 208

$168,171,172,173,175,184$, Transcendentalism, 33

185, 191, 196, 208, 224, $228 \quad$ Transdisciplinarity, 16

Sunni, 121, 126, 159, $204 \quad$ Transalectic, 29

Supervaluation, 52

Transfinite, 62

Syllogism, 56, 57, 117, 139, 166, Trialectic, 29

168, $203 \quad$ Tribe, 76, 78, 94

exclusive (Strong) Disjunctive, Trinity, 210, 216

57

hypothetical, 56, 57

inclusive (Weak) Disjunctive, 56

mixed hypothetical, 56

Symbol, 54, 55, 58, 159, 160, 166, $168,169,207$

fish-hook, 58

Syndrome, 38, 152

Dawn's, 152

Syntax, 55, 80, 135

Syriac, 112, 113

Tautology, 16

Testimony, 51, 100, 118, 153, 164, $169,177,195,220,221,223$

Theorem, 7, 50, 51, 71

Theory, 7, 8, 14, 16, 18, 19, 20, 24, $29,34,35,36,47,49,51,53,59$, $60,67,80,126,132,140,150$,

$151,159,212,213,215,216,217$ corpuscular, 59

Dempster-Shaffer, 8, 51, 67

Dezert - Smarandache, 8, 67

Dubois-Prade, 67

grand unified, 53

wave, 59

Yager, 67

Thesis, 17, 21, 22, 66

anti, 21

neo, 22

neuter, 22

non, 22

pre, 22

post, 22

Tripartition, 51, 52

Truth, 19, 26, 29, 30, 31, 35, 43, 46 $-51,53-56,60,62,64,89,90$, 91, 93, 95, 102, 105, 107, 115, $117,118,125,134,143,147$, $159,166-71,181,185,193$, 194, 195, 197, 199, 208, 212, $213,215,220,230$ absolute, 26, 35, 47, 48, 53, 54, $56,62,91,199$

false, 66 intangible absolute, 53 necessary, 53

possible, 53 relative, $26,48,53,54,91$ super, 55 true, 66

Umayyad, 103, 111, 112, 209

Unanimity or consensus (Ijma), $102,138,151,168,172,173$, $174,175,177,182,184,186$, 190, 192, 199, 206

Uncertainty, 46, 60, 206, 208

Uncountable, 54

Undecidability, 18

Undecided, 64

Underman, 42, 43

Uninformative, 19

Unlawful or Prohibited (Haram), $95,133,135,137,196,198,199$, 202, 208, 212

Unmatter, 8

Upanishad, 34 
Usury (Riba), 195, 198, 209

Vague, 17, 52, 58, 103,

Vagueness, 11, 45, 46, 64, 166, $168,208,220$

Valid, 52, 57, 58, 133, 135, 159, $173,175,176,177,178,188,189$ Validity, 49, 73, 160, 189, 190, 195, 197
Virtuous city, 139

Visishta Advaita, 34

Wisdom, 3, 4, 15, 113, 117, 135, 167, 179, 180, 181, 199, 200, 211 house of (Bayt al-Hikma), 113

Zealotism, 77, 148

Zoroastrianism, 113, 210

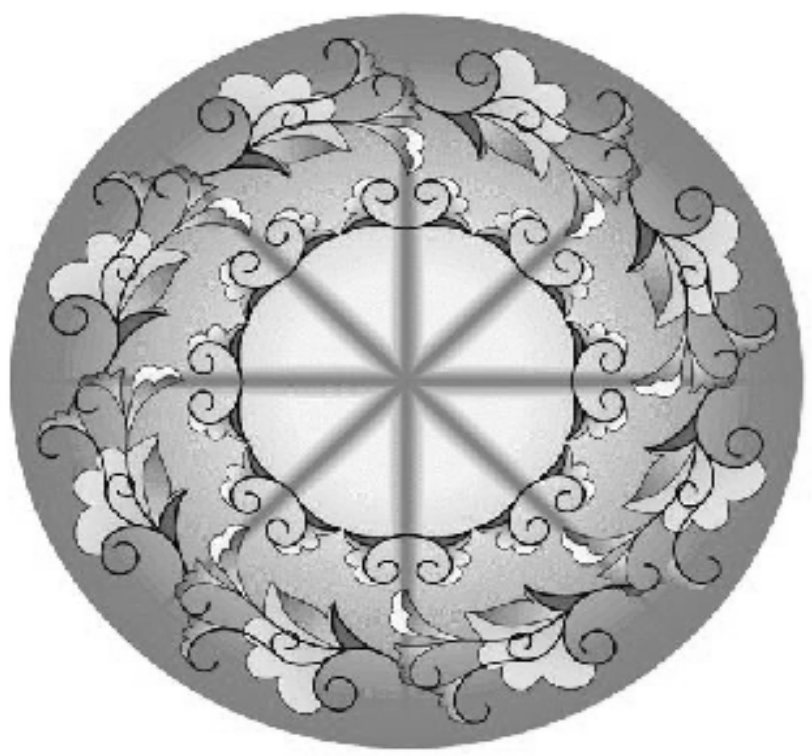




\section{References}

[1] Abd al-Karim Zidan, Summary of the Fundamentals of Jurisprudence (= Al-Wajiz fi ilm usul al-Fiqh), The Islamic House of distribution and publishing (Dar al-Tawzi'i wa alNashr al-Islamiyah), Cairo, 1993 (in Arabic).

[2] Abd Allah Ibn Ahmad Ibn Qudama al-Maqdisi, The Garden of Looker and the Paradise of Spectacles (= Rawdat alNadhir wa Janat al-Manadhir), ed. By Abd al-Aziz Abd alRahman Said, University of Imam Muhammad bin Massoud, Riyadh, second edition, 1978 (in Arabic).

[3] Abd al-Rahman Badawi, The History of Islamic Mysticism from the Beginning Until the End of the Second Century (= Tarikh al-Taswuf al-Islami: menn al-Bidaya hata Nihayit alQarn al-Thani al-Hijri), The Agency of Literature (Wakalt al-Matbu'at), Kuwait, 1975 (in Arabic).

[4] Abd al-Rahman Ibn Khaldun, The Introduction (= AlMuqaddimah), ed. by Ali Abdul Wahed Wafi, The Committee of Arabic Eloquence, second edition, Cairo, 1965 (in Arabic).

[5] Abu al-Qassim al-Shatibi, The Congruencies of the Sources of the Divine Law (= Al-Muwafaqa fi Usul al-Shari'a), The Major Trading Library, (Al-Maktaba al-Tijariyya al-Kura), Cairo, 1975 (in Arabic).

[6] Abu Hamid al-Ghazali, Incoherence of the Philosophers, (= Tahafut al-Falasifa), ed. by Sulyman Dunya, sixth edition, The House of Knowledge (Dar al-Ma'arif). Cairo, 1961 (in Arabic).

[7] Abu Hamid al-Ghazali, Logic of Incoherence of the Philosophers (= Mantiq Tahafut al-Falasifa al-Musama M'iyar al-Ilm), ed. by. Sulyman Dunya, The House of Knowledge (Dar al-Ma'arif). Cairo, 1961 (in Arabic).

[8] Abu Hamid al-Ghazali, The Chosen in The Science of the 
Origins of Jurisprudence (= al-Mustasfa), second edition, The House of Scientific Books (Dar al-Kutub al-Ilmya), Beirut, 1983(in Arabic).

[9] Abu Hayyan al-Tawhidi, Enjoyment and Conviviality (= AlImta wa al-Mu'anasa), ed. by Ahmad Amin and Ahmad Zein, The General Egyptian Book Organization (Al-Hay'ah al-Misriyah al-Ama li al-Kitab), Cairo (in Arabic).

[10] Abu Ishaq Ibrahim Ibn Ali al-Shirazi, The Bright Light in the Fundamentals of Jurisprudence (= Al-Luma' fi Ilm Usul al-Fiqh), ed. By Yusuf Badeiwi \& Mohi al-ddin Dib Mostawi, Ibn Kathir House (Dar Ibn Kathir), Beirut \& Damascus, second edition, 1997 (in Arabic).

[11] Abu Zakariya Yahya al-Nawawi, The Meadows of Virtuous from the Messenger's Speech (= Riyadd al-Saliheen menn Kalam Sayed al-Mursaleen), The Supplier Library (alMaktaba al-Imdadiyah), Mecca, without date (in Arabic).

[12] Ahmad Amin, Dawn of Islam (= Fagr al-Islam), The General Egyptian Book Organization (Al-Hay'ah alMisriyah al-A'ma li al-Kitab), Cairo, 1996 (in Arabic).

[13] Ahmad Amin, The Forenoon of Islam: the emergence of Science in the First Abbasid Age (= Duha al-Islam: Nash'at al-Ilum fi al-Asr al-Abasi al-Awal), The General Egyptian Book Organization (Al-Hay'ah al-Misriyah al-A'ma li alKitab), Cairo, 1998 (in Arabic).

[14] Ahmad Ibn Hajar al-Asqalani, Picketing and Sunnah (= AlI'itisam wa al-Sunnah), ed. by Khaled Abdel Fattah Shibl, The House of International Book \& The House of Egyptian Book (Dar al-Kitab al-Alami \& Dar al-Kitab al-Misri), Cairo, 1990 (in Arabic).

[15] Ahmad Ibn Muhammad Ibn Ya'qub Ibn Miskawayh, Politeness of Ethics and Clearing of Races (= Tahdhib alAkhlaq), ed. by Ibn al-Khatib, The Egyptian Press and its Library (Al-Matba'a al-Misriyyah wa Maktabatiha ), Cairo, Egypt, without date(in Arabic).

[16] Ahmad Mahmoud Subhi, On the Philosophy of History (Fi Falsaft al-Tarikh), The Establishment of University Culture (= Mu'asast al-Thaqafa al-Jami'iyah), Alexandria, 1975 (in Arabic).

[17] Ahmad Mahmoud Subhi, On the Science of Kalam: a 
Philosophical Study of the Opinions of Islamic sects in the Origins of Religion (= Fi ilm al-Kalam: dirassah falsafiyah li ar'a al-Firq al-Islamiyah fi usul al-Din), The Establishment of University Culture (Mu'asast al-Thaqafa al-Jami'iyyah), fourth edition, Alexandria, 1982 (in Arabic).

[18] Ahmad Mahmoud Subhi, Between the Principles of Authentication and the Principles of Historio-graphy: a Comparative Study in Methodology (= Bayn Usul al-Tahdith wa Usul al-Ta'arikh), in his "On the Philosophy of History" (Fi Falsaft al-Tarikh), OP. Cit., pp. 302 - 42 (in Arabic).

[19] Ahmad Mahmoud Subhi, This is My Book, Read it (= Ha'um Iqra'u Kitabiyyah), Library of Arab Raising (Maktabat alNahda al-arabiyyah), Beirut, 1997 (in Arabic).

[20] Ahmad Mahmoud Subhi \& Muhammad Ahmad Abdul Qadir, The Role of the Arabs in the Field of Sciences (= Dur al-Arab fi Majal al-Ulum), The House of University Knowledge (Dar al-Ma'rifa al-Jam'iya), Alexandria, Egypt, 1985(in Arabic).

[21] Albee, Ernest, History of English Utilitarianism, Collier Books, Crowell-Collier Publ. Co., N.Y., 1962.

[22] Ali Abu Milhem, Arab Philosophy: Problems and Solutions (Al-Falsafa al-Arabiyah: Mushkilat wa Hulul), Izz al-Din Establishment for Printing and Publishing (= Mu'asast Izz al-Din li al-Tiba'ah wa al-Nashr), Beirut, 1994 (in Arabic).

[23] Ali Sami Al-Nashar, Methodology of the Islamic Thinkers and the Discovery of the Scientific Method in the Islamic World (= Manahij al-Bahth ind Mufakiri al-Islam wa Iktishaf al-Minhaj al-Ilmi), The House of Knowledge (Dar al-Ma'arif), Cairo, 1967 (in Arabic).

[24] Ali Sami Al-Nashar, The Emergence of Philosoph- ical Thought in Islam (= Nash'at al-Fikr al-Falsa fi fi al-Islam), eighth edition, The House of Knowledge (Dar al-Ma'arif), Cairo, 1981 (in Arabic).

[25] American Romanian Academy, Romanians in the Western Science and Culture, second edition, Editor Dan Grindea, Davis, CA, USA, 1996, pp. 368 - 369.

[26] Arabic Language Academy, The Concise Dictionary (AlMu'ajam al-Wajiz), Prefaced by Ibrahim Bayoumi Madkour, Ministry of Education, Egyptian, Cairo,1990 (in Arabic). 
[27] Ashbacher, Charles D., Exploring Some Specific Functional Mappings To Create Operators In The Neutrosophic Logic, mss., 1999.

[28] Atanassov, K., Burillo, P., Bustince, H., On the Intuitionistic Fuzzy Relations, Notes on Intuitionistic Fuzzy Sets, Vol. 1 (1995), No. 2, 87 - 92.

[29] Atanassov, K., Bustince, H., Burillo, P., Mohedano, V., A Method for Inference in Approximate Reasoning for the Onedimensional Case Based on Normal Intuitionistic Fuzzy Sets, Proceedings of the VI IFSA World Congress, Sao Paulo, Brazil, July 1995, Vol. 1, 149-152.

[30] Atanassov, K., Stoyanova, D., Cartesian Products Over Intuitionistic Fuzzy Sets, Methodology of Mathematical Modelling, Vol. 1, Sofia, 1990, 296-298.

[31] Atanassov, K., Stoyanova, D., Remarks on the Intuitionistic Fuzzy Sets. II, Notes on Intuitionistic Fuzzy Sets, Vol. 1 (1995), No. 2, 85 - 86.

[32] Ayer, A.J., Logical Positivism, The Free Press of Glencoe, New York, 1958.

[33] Badr al-Din Muhammad al-Zarakshi, The Surrounding Sea in the fundamentals of jurisprudence $(=\mathrm{Al}-\mathrm{Bahr}$ al-Muheit fi Usul al-Fiqh), ed. by Muhammad Muhammad Tamir, The House of scientific books (Dar al-Kutub al-Ilmiyyah), Beirut, 2000 (in Arabic).

[34] Bailey, Cyril, The Greek Atomists and Epicurus, Russell \& Russell, Inc., New York, 1964.

[35] Bartold, V. V., The History of Islamic Civilization, (= Tarikh al-Hadara al-Islamiyah), Trans. into Arabic by Hamza Tahir, fifth edition, The house of knowledge (Dar al Ma'arif), Cairo, 1983(in Arabic).

[36] Berlin, Isaiah (ed.), The Empiricists: John Locke, George Berkeley, David Hume, Dolphin Books, Doubleday \& Company, Inc., Garden City, N.Y., 1961.

[37] Bogolubov, N. N., Logunov, A. A., Todorov, I. T., Introduction to Axiomatic Quantum Field Theory, Translated from Russian by Stephen A. Fulling and Ludmila G. Popova, W. A. Benjamin, Inc., Reading, Massachusetts, 1975. 
[38] Bouvier, Alain, George, Michel, Dictionnaire des Mathématiques, sous la direction de François Le Lionnais, Presses Universitaire de France, Paris, 1979.

[39] Bridges, Douglas, Constructive Mathematics, Stanford Encyclopedia of Philosophy, editor Edward N. Zalta, http://plato.stanford.edu/mathematics-constructive/, 1997.

[40] Bouwsma, W.J., The Culture of Renaissance Humanism, American Historical Association, Washington, 1973.

[41] Bronowski, Jacob, The Ascent of Man (= Irtiqa al-Insan) Trans. into Arabic by Ahmed Mostajir as The Civilizational Development of Man (Al-Tatwur al-hadari li al-Insan), The General Egyptian Book Organization (Al-Hay'ah alMisriyah al-Ama li al-Kitab), Cairos, 1997.

[42] Brown, E. G., Arab Medicine (= Al-Teb al-Arabi), Trans. into Arabic by Ahmad Shawki, reviewed by Muhammad Abdel Halim al-Oqbi, The Establishment of record of Arab (Mu'asast Sijil al-Arab), Cairo,1966 (in Arabic).

[43] Buhaescu, T., On an Order Relation between Fuzzy Numbers and Fuzzy Functions Convexity, Itinerant seminar on functional equations, approximation and convexity, ClujNapoca, 1987, 85-90.

[44] Buhaescu, T., On Quasicontinuity of fuzzy functions, Analele Universitatii din Galati, Matematica, Fizica, Mecanica Teoretica, Fascicula II, Anul VI (XI), 1988, 5-7.

[45] Buhaescu, T., On the Convexity of Intuitionistic Fuzzy Sets, Itinerant Seminar on Functional Equations, Approximation and Convexity, Cluj-Napoca, 1988, 137-144.

[46] Buhaescu, T., Some Observations on Intuitionistic Fuzzy Rerelations, Itinerant Seminar on Functional Equations, Approximation and Convexity, Cluj-Napoca, 1989, 111-118.

[47] Buhaescu, T., Intuitionistic Fuzzy Numbres, Analele Universitatii Dunarea de Jos, Galati, Fascicula II, Anul VIII 1990, 47-53.

[48] Buhaescu, T., Nombres Fuzzy Intuitionistiques, Analele Universitatii Galati, fascicola II, 1990-1991, 1-2.

[49] Buhaescu, T., Interval Valued Real Numbers, Sesiunea de comunicari stiintifice, Constanta, 6-8 iunie 1991, 34.

[50] Buhaescu, T., Convex Structures on the Fuzzy Sets Class, Fuzzy Systems \& AI, Vol. 2, No. 3, 1993, 15-20. 
[51] Buhaescu, T., Linear Programming with Intuitionistic Fuzzy objective, Notes on Intuitionistic Fuzzy Sets, Vol. 1 (1995), No. 2, 130-131.

[52] Buhaescu, T., Linear Programming with Intuitionistic Fuzzy Objective, International Colloquy the Risk in Contemporary Economy, Galati, Romania, Nov. 10-11, 1995, 29-30.

[53] Burnet, John, Greek Philosophy: Thales to Plato, St. Martin's Press, Inc., New York, 1962.

[54] Burillo, Lopez P., Bustince Sola H., Entropy on Intuitionistic Fuzzy Sets and on Interval-Values Fuzzy Sets, Fuzzy Sets and Systems, Vol. 78 (1996), No. 3, 305-316.

[55] Burillo, P., Bustince, H., Mohedano, V., Some Definitions of Intuitionistic Fuzzy Number. First Properties, Proc. of the First Workshop on Fuzzy Based Expert Systems (D. Lakov, Ed.), Sofia, Sept. 28-30, 1994, 53-55.

[56] Burillo, P., Bustince, H., Algebraic Structures for Intuitionistic Fuzzy Sets, Fifth Sci. Session of the Mathematical Foundation of Artificial Intelligence Seminar, Sofia, October 5, 1994, Preprint MRL-MFAIS-10-94, Sofia, 1994, 1-13.

[57] Burillo, P., Bustince, H., Isoentropic Methods for Construction of IVFS, Proc. of the 4-th International Workshop CIFT'94, Trento, June 1-3, 1994, 57-60.

[58] Burillo, P., Bustince, H., Numerical Measurements of Information on Intuitionistic Fuzzy Sets and Interval-valued Fuzzy Sets ( $\$ \mid$ Phi\$ -fuzzy), Fifth Sci. Session of the Mathematical Foundation of Artificial Intelligence Seminar, Sofia, October 5, 1994, Preprint MRL-MFAIS-10-94, Sofia, 1994, 14-26.

[59] Burillo, P., Bustince, H., Two Operators on Interval-Valued Intuitionistic Fuzzy Sets: Part I, Comptes rendus de l'Academie Bulgare des Sciences, Tome 47, 1994, No. 12, 912.

[60] Burillo, P., Bustince, H., Informational Energy on Intuitionistic Fuzzy Sets and on Interval-Values Intuitionistic Fuzzy sets (\$\Phi -fuzzy). Relationship between the Measures of Information, Proc. of the First Workshop on Fuzzy Based Expert Systems (D. Lakov, Ed.), Sofia, Sept. 28-30, 1994, 46-49. 
[61] Burillo, P., Bustince, H., Numeros Intuicionistas Fuzzy, IV Congreso de la Asociacion Espanola de logica y Tecnologia Fuzzy, 1994, 97-103.

[62] Burillo, P., Bustince, H., Orderings in the Referential Set Induced by an Intuitionistic Fuzzy Relation, Notes on Intuitionistic Fuzzy Sets, Vol. 1 (1995), No. 2, 93-103.

[63] Burillo, P., Bustince, H., Two Operators on Interval-Valued Intuitionistic Fuzzy Sets: Part II, Comptes rendus de l'Academie Bulgare des Sciences, Tome 48, 1995, No. 1, 1720.

[64] Burillo, P., Bustince, H., Metodos Intuicionistas Fuzzy, V Congreso de la Asociacion Espanola de logica y Tecnologia Fuzzy, 1995, 147-153.

[65] Burillo, P., Bustince, H., Intuitionistic Fuzzy Relations. Part I, Mathware and Soft Computing, Vol. 2 (1995), No. 1, 5-38.

[66] Burillo, P., Bustince, H., Intuitionistic Fuzzy Relations. Part II, Mathware and Soft Computing, Vol. 2 (1995), No. 2, 117-148.

[67] Burillo, P., Bustince, H., Construction Theorems for Intuitionistic Fuzzy Sets, Fuzzy Sets and Systems, Vol. 84, 1996, No. 3, 271-281.

[68] Burnet, John, Greek Philosophy: Thales to Plato, St. Martin's Press, Inc., New York, 1962.

[69] Bustince, H., Numerical Information Measurements in Intervalvalued Intuitionistic Fuzzy Sets (IVFS), Proc. of the First Workshop on Fuzzy Based Expert Systems (D. Lakov, Ed.), Sofia, Sept. 28-30, 1994, 50-52.

[70] Bustince, Sola H., Conjuntos Intuicionistas e Intervalovalorados Difusos: Propiel-dades y Construccion. Relaciones Intuicionistas y Estructuras, Ph. D., Univ. Publica de Navarra, Pamplona, 1994.

[71] Bustince, H., Correlation of Interval-Valued Intuitionistic Fuzzy Sets, Fifth Sci. Session of the Mathematical Foundation of Artificial Intelligence Seminar, Sofia, October 5, 1994, Preprint MRL-MFAIS-10-94, Sofia, 1994, 27-35.

[72] Bustince, H., Handling Multicriteria Fuzzy Decision Making Problems Based on Intuitionistic Fuzzy Sets, Notes on Intuitionistic Fuzzy Sets, Vol. 1, No. 1, 1995, 42-47.

[73] Bustince, H., Burillo P., A Theorem for Constructing Interval Valued Intuitionistic Fuzzy Sets from Intuitionistic Fuzzy 
Sets, Notes on Intuitionistic Fuzzy Sets, Vol. 1, No. 1, 1995, 516.

[74] Bustince, H., Burillo, P., Antisymmetrical Ituitionistic Fuzzy Relation - Order on the Referential Set Induced by an Intuitionistic Fuzzy Relation, BUSEFAL Vol. 62, 1995, 17-21.

[75] Bustince, H., Burillo, P., Correlation of Interval-Valued Intuitionistic Fuzzy Sets, Fuzzy Sets and Systems, Vol. 74 (1995), No.2, 237-244.

[76] Bustince, H., Burillo, P., Mohedano, V., A Method for Inference in Approximate Reasoning Based on Normal Intuitionistic Fuzzy Sets, Notes on Intuitionistic Fuzzy Sets, Vol. 1, No. 1, 1995, 51-55.

[77] Bustince, H., Burillo, P., Vague Sets are Intuitionistic Fuzzy Sets, Fuzzy Sets and Systems, Vol. 79, 1996, No. 3, 403-405.

[78] Bustince, H., Mohedano, V., About the Intuitionistic Fuzzy Set Generators, Proceedings of the First International Conference on Intuitionistic Fuzzy Sets (J. Kacprzyk and K. Atanassov Eds.), Sofia, Oct 18-19, 1997; Notes on Intuitionistic Fuzzy Sets, Vol. 3 (1997), No. 4, 21-27.

[79] Bustince, H., Mohedano, V., About the Complement in Intuitionistic Fuzzy Sets, Notes on Intuitionistic Fuzzy Sets, Vol. 3 (1997), No. 1, 12-19.

[80] Carr, M. H., Realists and Nominalists, Oxford University Press, Fair Lawn, NJ, 1946.

[81] Cartwright, John, Evolution and Human Behavior: Darwinism Perspectives on Human Nature, Macmillan Press, LTD, London, 2000.

[82] Copleston, Frederick, Arthur Schopenhauer, Philosopher of Pessimism, Barnes and Noble Books, New York, 1975.

[83] Dempster, A. P., Upper and Lower Probabilities Induced by a Multivalued Mapping, Annals of Mathematical Statistics, 38, 325-339, 1967.

[84] Dezert, J., Autonomous Navigation with Uncertain Reference Points Using the PDAF, In Multitarget-Multisensor Tracking: Applications and Advances, Volume 2, Yaakov Bar-Shalom Editor, pp 271-324, 1992.

[85] Dezert, Jean, On a Problem of Autonomous Navigation of an Engine Car (approximate title), Ph. D. thesis, ONERA, Paris, 1990. 
[86] Dezert, J., Vers un Nouveau Concept de Navigation Autonome D'engin; Un Lien Entre la Théorie de L'évidence et le Filtrage à Association Probabiliste de Données, Ph. D. Thesis, no 1393, University Paris 11, Orsay, France, Sept. 1990.

[87] Dimitrov, D., Atanassov, K., Shannon, A., Bustince, H., Kim, S.-K., Intuitionistic Fuzzy Sets and Economic Theory, Proceedings of The Second Workshop on Fuzzy Based Expert Systems, FUBEST'96 (D. Lakov, Ed.), Sofia, Oct. 9-11, 1996, 98-102.

[88] Dinulescu-Câmpina, Gheorghe, The Intangible Absolute Truth, Smarandache Notions Journal, 142-143, 2000.

[89] Dummett, M., Wang's Paradox, Synthese, 30, 301-324, 1975.

[90] Dunn, J. M., Intuitive Semantics for First Degree Entailment and Coupled Trees, Philosophical Studies, Vol. XXIX, pp. 149-68, 1976.

[91] Fatehia Al-Nabrawi \& Muhammad Nasr Mihana, The Development of Political Thought in Islam: a Comparative Study (= Tatwur al-Fikr al-Siyasi fi al-Islam:Dirassa Muqarinah), Vol. 1, The House of Knowledge (Dar alMa'arif), Cairo, 1982 (in Arabic).

[92] Fine, K., Vagueness, Truth and Logic, Synthese, 30, 265300, 1975.

[100] Fisch, Max, and Turquette, Atwell, Pierce's Triadic Logic, Transactions of the Charles S. Peirce Society, 11, 71-85, 1966.

[101] Frege, Gottlob, On Sense and Meaning, In Peter Geach \& Max Black (ed.), Translations from the Philosophical Writings of Gottlob Frege, Barnes\& Noble books, Totowa, N.J., Reprinted 1988, pp. $56-80$.

[102] Fuad Muhammad Fakhr al-Din, The Future of Muslims (= Mustaqbal al-Muslimeen), The Literatures of People (Matbu'at al-Sha'ab), Cairo, 1976(in Arabic).

[103] Fulbright, William, Arrogance of Power (= Ghatrasat alQuwa), trans. into Arabic by Mahmoud Shukri al-Adawi, The House of Arab Writer for Printing and Publishing (Dar al-Katib al-Arabi li al-Tiba'a wa al-Nashr), Cairo, without date (in Arabic). 
[104] Girard, Jean-Yves, Linear Logic, Theoretical Computer Science, 50:1-102, 1987.

[105] Goldberg, Samuel, Probability / An Introduction, PrenticeHall, Inc., Englewood Cliffs, NJ, 1960.

[106] Goguen, J. A., The Logic of Inexact Concepts, Synthese, 19, 325-375, 1969.

[107] Halldén, S., The Logic of Nonsense, Uppsala Universitets Arsskrift, 1949.

[108] Hamza Tahir, the Arabic translation of Bartold's The History of Islamic Civilization, fifth edition, The house of knowledge (Dar al Ma'arif), Cairo, 1983, pp. $1-15$.

[109] Hassan Abd al-Hamid \& Muhammad Mahran, On the Philosophy of Science and methodology (= Fi Falsfat alUlum wa Manahij al-Bahth), Said R'afat Library (Maktabat Said R'afat), Cairo 1979 / 1980 (in Arabic).

[110] Hassing, Richard F., Final Causality in Nature and Human Affairs, The Catholic University of America Press, Baltimore, 282 p., 1997.

[111] Hegel, G.W.F., The Phenomenology of Spirit, trans., A.V.Miller, Clarendon Press, Oxford, 1977.

[112] Heitkoetter, Joerg; David Beasley, David, The Hitch-Hiker's Guide to Evolutionary Computing, Encore, http://surf.de.uu.net/encore/.

[113] Hellerstein, N. S., DELTA, A Paradox Logic, World Scientific, Singapore, New Jersey, London, Hong Kong, 1999.

[114] Hobbes, Thomas, Body, Man and Citizen, Collier Books, Crowell-Collier Publishing Co., New York, 1962.

[115] Hoffmann, Banesh, The Strange Story of the Quantum, An Account for the General Reader of the Growth of the Ideas Underlying our Present Atomic Knowledge, Dover Publications, Inc., New York, 1959.

[116] Hogg, Robert V., Craig, Allen T., Introduction to Mathematical Statistics, Second edition, The Macmillan Company, New York, 1969.

[117] Husni Ahmad Al-Saiyed Hammad, Arab Civilization: its Inception, its Evolution, its Effects (= Al-Hadarrah alArabiyah: Nash'atiha, Tatwuriha, Atharaha), The Establishment of Compilation an Publication \& The House of Arab writer (Mu'asast al-Ta'alif wa al-Nashr \& Dar al- 
Katib al-Arabi), Cairo, 1967 (in Arabic).

[118] Hyde, Dominic, Sorites Paradox, Stanford Encyclopedia of Philosophy, edited by Edward N. Zalta, 1996, http://plato.stanford.edu/entries/sorites-paradox/.

[119] Illingworth, Valerie, The Penguin Dictionary of Physics, second edition, Penguin Books, 1991.

[120] Imam al-Haramayn Ibn al-Juwayni, The Proofs of Jurisprodence (= Al-Burhan fi Usul al-Fiqh), ed. by Abd alAdhim al-Dib, The House of al-Ansar (Dar al-Ansar), Cairo, 1980 (in Arabic).

[121] Imam al-Shafi'I, Al-Risalah for the authoritative position of the Sunnah, ed. By Ahmad Muhammad Shakir, The Scientific House of Books (Dar al-Kutub al-Ilmiyyah), Beirut, 1992 (in Arabic).

[122] Iorga, Nicolae, Cugetări, edited by Elisabeta Jurca-Pod, The Yellow Bird Publ., Chicago, 1991.

[123] Jaspers, K., Nietzsche: An Introduction to the Understanding of His Philosophical Activity, University of Arizona Press, Tucson, 1965.

[124] Jaspers, Karl, General Psychopathology, translated by J. Hoenig and Marian W. Hamilton, Introduction by Paul McHugh, The John Hopkins University Press, Baltimore, Vol. I and II.

[125] Kant, Immanuel, Critique of Pure Reason, St. Martin's Press, New York, 1965.

[126] Kasabov, N., Foundations of Neural Networks, Fuzzy Systems and Knowledge Engineering, MIT Press, 1996.

[127] Kenny, A., Aquinas, Hill and Wang, Inc., New York, 1980.

[128] Klein, Felix, Vergleichende Betrachtungen Über neuere geometrische Forschungen, Mathematische Annalen, 43, 63-100, 1893.

[129] Kessler, John, Giordano Bruno: the Forgotten Philosopher, http://www.infidels.org/library/historical/john kessler/giord ano bruno.html

[130] Kockelmans, J.L., Phenomenology: The Philosophy of Edmund Husserl and Its Interpretation, Doubleday and Company, Inc., Garden City, N.Y., 1967.

[131] Korner, S., The Philosophy of Mathematics, Hutchinson, London, 1960. 
[132] Lambert, J. H., Neues Organon, Leipzig, 1764.

[133] Lambert, J. H., Philosophische Schriften, Vol.I \& II, reprinted by Olms, 1985.

[134] Le, Charles T., The Smarandache Class of Paradoxes, in Journal of Indian Academy of Mathematics, Bombay, India, No. 18, 53-55, 1996.

[135] Leff, Gordon, Medieval Thought from St. Augustine to Ockham, Penguin Books, Inc., Baltimore, 1962.

[136] Lewis, John, Man and Evolution, trans. into Arabic by Saleh Jawad Kadhim as The Man, that the Unique Creature (AlInsan, Zalik al-Ka'in al-Farid), The General Egyptian Book Organization (Al-Hay'ah al-Misriyah al-A'ma li al-Kitab), Cairo \& the House of Cultural affairs (Dar al-Shu'un alThaqafiyah), Baghdad, 1986.

[137] Lin, T. Y., E-mails to C. T. Le, August 1999.

[138] Loeb, Peter A. \& Wolff, Manfred (eds.). Nonstandard Analysis for the Working Mathematician, [B] Mathematics and its Applications, Dordrecht, Kluwer Academic Publishers. xiv, 311 p., 2000.

[139] Mackey, George W., Mathematical Foundations of Mechanics I A Lecture-Note Volume, The Benjamin/Cummings Publishing Company, Reading, Massachusetts, 1980.

[140] Mahmoud al-Shafi'i, The introduction to the study of Kalam (= Al-Madkhal ila Dirasit Ilm al-Kalam), second edition, Wahba Library (Maktabt Wahba), Cairo, 1991 (in Arabic).

[141] Mahmoud Fahmi Zeidan, On the Philosophy of Language (= Fi Falsa fat al-Lughah), The House of Arab Raising (Dar alNahda al-Arabiyyah), Beirut, 1985 (in Arabic).

[142] Marcel, Gabriel, Man against Mass Society, Henry Regnery Co., Chicago, 1962.

[143] Marcuse, Herbert, Reason and Revolution: Hegel and the Rise of Social Theory, Beacon Press, Boston, 1960.

[144] Mathematical Logic Around The World, University of Bonn, Germany, http://world.logic.at/.

[145] McKeon, Richard P., An Introduction to Aristotle, Random House, Inc., New York, 1947.

[146] McNeil, Martin, F., Thro, Ellen, Fuzzy Logic / A Practical Approach, Foreword by Ronald R. Yager, Academic Press, 1994. 
[147] Mehta, J. L., Martin Heidegger: The Way and the Vision, University of Hawaii Press, Honolulu, 1976.

[148] Mendenhall, William, Introduction to Probability and Statistics, Fourth edition, Duxbury Press, North Scltuate, Massachusetts, 1975.

[149] Mortensen, Chris, Inconsistent Mathematics, Stanford Encyclopedia of Philosophy, editor Edward N. Zalta, http://plato.stanford.edu/entries/mathematics-inconsistent/, 1996.

[150] Moschovakis, Joan, E-mails to C. T. Le, August 1999.

[151] Muhammad Ahmad Abd al-Qader, Divine Science and its Effects on Thought and Reality (= Al-Ilm al-Ilahi wa Atharuh fi al-Fikr wa al-Waq'I), The House of University Knowledge (Dar al-Ma'rifa al-Jami'iyah), Alexandria, Egypt, 1996 (in Arabic).

[152] Muhammad Al-Ghazali, A Defense of the Faith and Shari'a Against the Stabs of Orientalists (= Dif'a an al-Aqida wa al_Shari'a ded Mata'in al-Mustashriqin), fourth edition, The House of Modern Books (Dar al-Kutub al-Hadithah), Cairo, 1975 (in Arabic).

[153] Muhammed Aly Abu Rayan, History of Greek Thought:Greek Philosophy from Thales to Plato (= Tarikh al-Fikr al-Younani: al-Falsafa al-Younaniyah menn Tales ila Aflaton), The House of University Knowledge (Dar alMa'rifa al-Jam'iya), Alexandria, Egypt, 1988 (in Arabic).

[154] Muhammed Ibn Abu Bakr al-Razi, The Chosen of Correction Dictionary, (= Mukhtar al-Sahah), ed. by Mahmoud Khater, The House of Hadith (Dar al-Hadith), Cairo, without date (in Arabic).

[155] Muhammad Ibn Ali al-Shawkani, Guidance of the Masters to the Realization of the Right of the Science of Origins, (= Irshad al-Fuhul ila Tahqiq al-Haqq min Ilm al-Usul), ed. by Ahmad Inayyah, The House of Arab Book for Publishing and Distribution, (Dar al-Kitab al-Arabi li al-Nashr wa alTawzi') Beirut, 2002 (in Arabic).

[156] Muhammad Mustafa Hilmi, The Spiritual Life in Islam (= Al-Hayatu al-Ruhiyah fi al_Islam), The General Egyptian Book Organization (Al-Hay'ah al-Misriyah al-Ama li alKitab), Cairo, 1984 (in Arabic). 
[157] Muhammad Shama, Islam in the Western Thought (= alIslam fi al-Fikr al-Gharbi), Wahba Library (Maktabt Wahba), Cairo, 1980 (in Arabic).

[158] Muhammad Thabit al-Findi, Philosophy of Mathematics (= Falsafat al-Riyyadah), The House of University Knowledge (Dar al-Ma'rifah al-Jami'iyyah), Alexandria, 1990 (in Arabic).

[159] Munshi, K.M., Diwakar, R.R. (gen. eds.), Introduction to Vedanta, by P. Nagaraja Rao, Bhavan's Book University, Chowpatty, Bombay, India, 1966.

[160] Mustafa al-Siba'i, Sunnah and its Place in Islamic legislation (= Al-Sunnah wa Makanatiha fi al-Fiqh alIslami), The Islamic Library for Printing and Publishing (AlMaktabh al-Islami li al-Tiba'a wa al-Nashr), Beirut \& Damascus, 1978 (in Arabic).

[161] Nabil Aly, Arabic Culture and the Age of Information: a View of the Future of Arabic Culture Speech (= Al-Thaqafa al-Arabiyah wa Asr al-Ma'lumat), Series of the world of knowledge (Silslat A'lam al-Ma'rifa), the National Council for Culture, Arts and Literatures, Kuwait, No. 292, December 2001(in Arabic).

[162] Narinyani, A., Indefinite Sets - a New Type of Data for Knowledge Representation, Preprint 232, Computer Center of the USSR Academy of Sciences, Novosibirsk, 1980 (in Russian).

[163] Nasir Ibn Sulyman al-Omar, The Difference in Islamic Action: Causes and Effects (= Al-Ikhtilaf fi al-Amal alIslami: al-Asbab wa al-Nata'ij), a paper presented to the scientific conference entitled: 'Islamic Action between Agreement and Disagreement', University of Khartoum, Department of Islamic Culture, 12 - 14 July 2004. pp. 1 38. (in Arabic).

[164] Nobre, Farley Simon M., E-mails to M. Perez, August 1999.

[165] Peirce, C.S., Essays in the Philosophy of Science, The Liberal Arts Press, Inc., New York, 1957.

[166] Peirce, C. S., Essays in the Philosophy of Science, The Liberal Arts Press, Inc., New York, 1957.

[167] Popa, Constantin M., The Paradoxist Literary Movement, Xiquan Publ., Hse., Phoenix, 1992. 
[168] Priest, Graham; Tanaka, Koji, Paraconsistent Logic, Stanford Encyclopedia of Philosophy, editor Edward N. Zalta, http://plato.stanford.edu/entries/logic-paraconsistent/.

[169] Priest, Graham, Dialetheism, Stanford Encyclopedia of Philosophy, ed. by Edward N. Zalta, http://plato.stanford.edu/entries/dialetheism/.

[170] Quine, W. V., What Price Bivalence?, Journal of Philosophy, 77, 90-95, 1981.

[171] Rescher, N., Many-Valued Logic, McGrow-Hall, N.Y., 1969.

[172] Robinson, A., Non-Standard Analysis, Princeton University Press, Princeton, NJ, 1996.

[173] [194] Rousseau, Jean-Jacques, On the Social Contract, trans. Judith R. Masters, St. Martin's Press, Inc., New York, 1978.

[174] Routley, Richard and Val, The Semantics of First Degree Entailment, Nôus, Vol. 6, 335-359, 1972.

[175] Rugina, Anghel N., Geldtypen und Eldordnungen. Fundamente für eine echte allgemeine Geld und Wirtschaftstheorie, W. Kohhammer Verlag, Stuttgard, Germany, 1949.

[176] Rugina, Anghel N., What is the Alternative for the West, International Journal of Social Economics, Vol. 8, No. 2, 1981.

[177] Rugina, Anghel N., Principia Methologica 1: A Bridge from Economics to all Other Natural Sciences / Towards a Methodological Unification of all Sciences, MCB University Press Ltd., 1989.

[178] Rugina, Anghel N., Prolegomena to any Future Study in Economics, Finance and Other Social Sciences: The Road to a Third Revolution in Economic, Financial, Social, Ethical, Logical and Political Thinking, <International Journal of Social Economics>, Vol. 25, No. 5, 1998.

[179] Runes, Dagobert D., Dictionary of Philosophy, A Helix Book, Rowman \& Allanheld, Totowa, New Jersey, 1984.

[180] Russell, Bertrand, Introduction to Mathematical Philosophy, Dover Publications, Inc., New York, 1993.

[181] Ryle, Gilbert, The Concept of Mind, Barnes and Noble, Inc., New York, 1950. 
[182] Sabra, Avicenna on the Subject Matter of Logic, The Journal of Philosophy, Inc., 1980, pp. $746-764$.

[183] Sabra, The Appropriation and Subsequent, Naturalization of Greek Science in Medieval Islam: A Preliminary Statement, History of science, Volume 25, Part 3, Number 69, September 1987, pp. $223-243$.

[184] Sabra, Science and Philosophy in Medieval Islamic Theology: The Evidence of the Fourteenth Century, Zeitschrift für geschichte der arabisch - Islamischen wissenschaften, Sonderdruch, Band 9, 1994, pp. 1 - 42.

[185] Sabra, A.I., Situating Arabic Science: Locality versus Essence, Isis, 87, 1996, pp. $654-670$.

[186] Salah Osman, Darwinism and Human: the Theory of Evolution from Science to Globalization (= Al-Darwiniyah wa al-Insan: Nazariyt al-Tatwur menn al-Ilm ila alAwlama), Establishment of Knowledge (Munsha't alMa'arif), Alexandria, Egypt, 2001(in Arabic).

[187] Salah Osman, Scientific Model between Reality and Imagination (= Al-Namuzaj al-Ilmi bayn al-Khayal wa alWaqi'), Establishment of Knowledge (Munsha't al-Ma'arif), Alexandria, Egypt, 2001(in Arabic).

[188] Salah Osman, Many-Valued Logic between the Degrees of Truth and the Limits of Knowledge (= Al-Mantiq Mut'adid al-Qiyyam bayn Darajat al-Sidq wa Hudud al-M'arifa), Establishment of Knowledge (Munsha't al-Ma'arif), Alexandria, Egypt, 2002 (in Arabic).

[189] Salah Osman, The Illusion of External World Between Language and Cognition (= Wahm al-Alam al-Khariji bayna al-Lugha wa al-Idrak), The Establishment of Knowledge (Munsha't al-Ma'arif), Alexandria, Egypt, 2004 (in Arabic).

[190] Salah Osman, Dialectic of Constancy and Motion in Zeno's Paradoxes: a Contemprary Mathematical View (= Jadal alThabat wa a-Haraka bayn Darjat al-Sidq wa Hudud alM]arifa), Journal of Facult of Arts , Menoufia University, Egypt , (58), July 2004,pp. 99 -139 (in Arabic).

[191] Sartre, Jean-Paul, Existentialism and Human Emotions, Philosophical Library, Inc., New York, 1957. 
[192] Scruton, Roger, A Short History of Modern Philosophy / From Descartes to Wittgenstein, Routledge, London, 1992.

[193] Shafer, Glenn, A Mathematical Theory of Evidence, Princeton University Press, NJ, 1976.

[194] Shafer, Glenn, The Combination of Evidence, International Journal of Intelligent Systems, Vol. I, 155-179, 1986.

[195] Sharif al-Chaoubachi: Long Live the Arab Language, Down Sibawayh (= Tahiya al-Lugha al-Arabiyah, Yasqut Sibawayh), The General Egyptian Book Organization (AlHay'ah al-Misriyah al-Ama li al-Kitab), Cairo, 2004 (in Arabic).

[196] Smarandache, Florentin, A Unifying Field in Logics: Neutrosophic Logic. / Neutrosophic Probability, Neutrosophic Set, Preliminary report, Western Section Meeting, Santa Barbara, CA, USA, Meeting \# 951 of the American Mathematical Society, March 11-12, 2000, http://www.ams.org/amsmtgs/2064 presenters.html and http://www.ams.org/amsmtgs/2064 program saturday.html.

[197] Smarandache, Florentin, Collected Papers, Vol. II, University of Kishinev Press, Kishinev, 1997.

[198] Smarandache, Florentin. Collected Papers, Vol. III, Abaddaba, Oradea, 160 p., 2000.

[199] Smarandache, Florentin, Distihuri Paradoxiste, Dorul, Norresundby, 1998.

[200] Smarandache, Florentin, Linguistic Paradoxists and Tautologies, Libertas Mathematica, University of Texas at Arlington, Vol. XIX, 143-154, 1999.

[201] Smarandache, Florentin, Neutrosophic Logic, A Generalization of the Fuzzy Logic, http://www.gallup.unm.edu/ $\sim$ smarandache/NeutLog.txt.

[202] Smarandache, Florentin, Neutrosophic Probability, A Generalization of the Classical Probability, http://www.gallup.unm.edu/ smarandache/NeutProb.txt.

[203] Smarandache, Florentin, Neutrosophic Set, A Generalization of the Fuzzy Set, http://www.gallup.unm.edu/ smarandache/NeutSet.txt.

[204] Smarandache, Florentin, Neutrosophy, A New Branch of Phylosophy, http://www.gallup.unm.edu/ smarandache/NeutroSo.txt. 
[205] Smarandache, Florentin, Neutrosophy: Neutrosophic Probability, Set, and Logic, American Research Press, Rehoboth, USA, 105p., 1998.

[206] The Florentin Smarandache Papers, Special Collection, Archives of American Mathematics, Center for American History, SRH 2.109, University of Texas at Austin, TX 78713, USA.

[207] The Florentin Smarandache Papers, Special Collection, Arizona State University, Hayden Library, Tempe, AZ 85287, USA.

[208] Soare, Ion, Un Scriitor al Paradoxurilor: Florentin Smarandache, Almarom, Rm. Vâlcea, 1994.

[209] Soare, Ion, Paradoxism si Postmodernism, Almarom, Rm. Vâlcea, 2000.

[210] Sonnabend, Thomas, Mathematics for Elementary Teachers, Second Edition, Saunders College Publishing, 1997.

[211] Song, Feijun, E-mail to C. T. Le, August 1999.

[212] Southern, R.W., Western Views of Islam in the Middle Ages, Harvard university press, Mass./ London, 1978.

[213] Stephens, J., Francis Bacon and the Style of Science, University of Chicago Press, Chicago, 1975.

[214] Stojmenovic, Ivan, editor, Many-Valued Logic, on-line journal, E-mails to C. T. Le, August 1999.

[215] Stoyanova, D., A Variant of a Cartesian Product over Intuitionistic Fuzzy Sets, Second Sci. Session of the Mathematical Foundation of Artificial Intelligence Seminar, Sofia, March 30, 1990, Prepr. IM-MFAIS-1-90, 43-45.

[216] Stoyanova, D., Algebraic Structures of Intuitionistic Fuzzy Sets, Third Sci. Session of the Mathematical Foundation of Artificial Intelligence Seminar, Sofia, June 12, 1990, Preprint IM-MFAIS-2-90, Part 1, 19-21.

[217] Stoyanova, D., Algebraic Structures of Fuzzy Sets, Third Sci. Session of the Mathematical Foundation of Artificial Intelligence Seminar, Sofia, June 12, 1990, Preprint IMMFAIS-2-90, Part 1, 15-18.

[218] Stoyanova, D., Sets from (\$lalpha, lbeta\$) - Level Generated by an Intuitionistic Fuzzy Sets, Ninetieth Session of the Nat. Seminar of Informatics of the Union of Bulg. Mathematicians and Fourth Scientific Session of the 
Mathematical Foundation Artificial Intelligence Seminar, Sofia, Nov. 5, 1990, Preprint IM-MFAIS-5-90, Sofia, 1990, $40-42$.

[219] Stoyanova, D., Atanassov K., Relations between Operators, Defined over Intuitionistic Fuzzy Sets, Second Sci. Session of the Mathematical Foundation of Artificial Intelligence Seminar, Sofia, March 30, 1990, Prepr. IM-MFAIS-1-90, 4649.

[220] Stoyanova, D., Compositions of Intuitionistic Fuzzy Relations, BUSEFAL Vol. 54, 1993, 21-23.

[221] Stoyanova, D., More on Cartesian Products over Intuitionistic Fuzzy Sets, BUSEFAL Vol. 54, 1993, 9-13.

[222] Suber, Peter, Glossary of First-Order Logic, Philosophy Department, Earlham College, http://www.earlham.edu/ peters/courses/logsys/glossary.ht $\underline{\mathrm{m}}, 1999$.

[223] Sulyman Dunya, the introduction of the sixth edition of alGhazali's Incoherence of the Philosophers, The House of Knowledge (Dar al-Ma'arif). Cairo, 1961, pp. 7 - 70 (in Arabic).

[224] Tabirca, Sabin, Some Recent Results Concerning the Smarandache Type Notions, Conference held at the Department of Mathematics, Manchester University, England, 15 May 2000.

[225] TeSelle, E., Augustine the Theologian, Herder \& Herder, Inc., 1970.

[226] Tawfiq al-Tawil, On our Arab-Islamic Heritage (= Fi Turathina al-Arabi al-Islami), Series of the world of knowledge, the National Council for Culture, Arts and Literatures, Kuwait, No. 87, March 1985(in Arabic).

[227] Troelstra, Anne S., Lectures on Linear Logic, CSLI Lecture Notes 29, Center for the Study of Language and Information, Stanford, California, 1992.

[228] Tanaka, Koji, Philosophy Department, University of Queensland, Brisbane, Australia, E-mails to Minh Perez, USA, August 1999.

[229] Torretti, Roberto, Nineteenth Century Geometry, in Stanford Encyclopedia of Philosophy, edited by Edward N. Zalta, http://plato.stanford.edu/entries/geometry-19th/\#1, 1999. 
[230] Tye, M., Sorites Paradoxes and the Semantics of Vagueness, in Philosophical Perspectives: Logic and Language, edited by J. Tomberlin, Ridgeview, Atascadero, USA, 1994.

[231] Van Fraassen, B. C., The Scientific Image, Clarendon Press, 1980.

[232] Vasiliu, Florin, Paradoxism's Main Roots, Ed. Haiku, Bucharest, 1994.

[233] Veatch, H.B., A Contemporany Appreciation, Indiana University Press, Bloomington, 1974.

[234] Vlastos, Gregory, The Philosophy of Socrates, Anchor Books, Garden City, New York, 1971.

[235] Weisstein, Eric W., CRC Concise Encyclopedia of Mathematics, CRC Press, Boca Raton, p. 1806, 1998.

[236] Williamson, Timothy, Vagueness, Routledge, London \& N.Y., 1994.

[237] Wittgenstein, L., Tractatus Logico-Philosophicus, Humanitas Press, New York, 1961.

[238] Zadeh, Lotfi A., Fuzzy Logic and Approximate Reasoning, Synthese, 30, 407-428, 1975.

[239] Zadeh, Lotfi A., Reviews of Books (A Methematical Theory of Evidence. Glenn Shafer, Princeton University Press, Princeton, NJ, 1976), The AI Magazine, 81-83, 1984.

[240] Zaki Naguib Mahmoud, The renewal of the Arab Thought (= Tajdid al-Fikr al-Arabi), Sixth Edition, The House Of alShurouk (Dar al-Shurouk), Cairo, 1980.

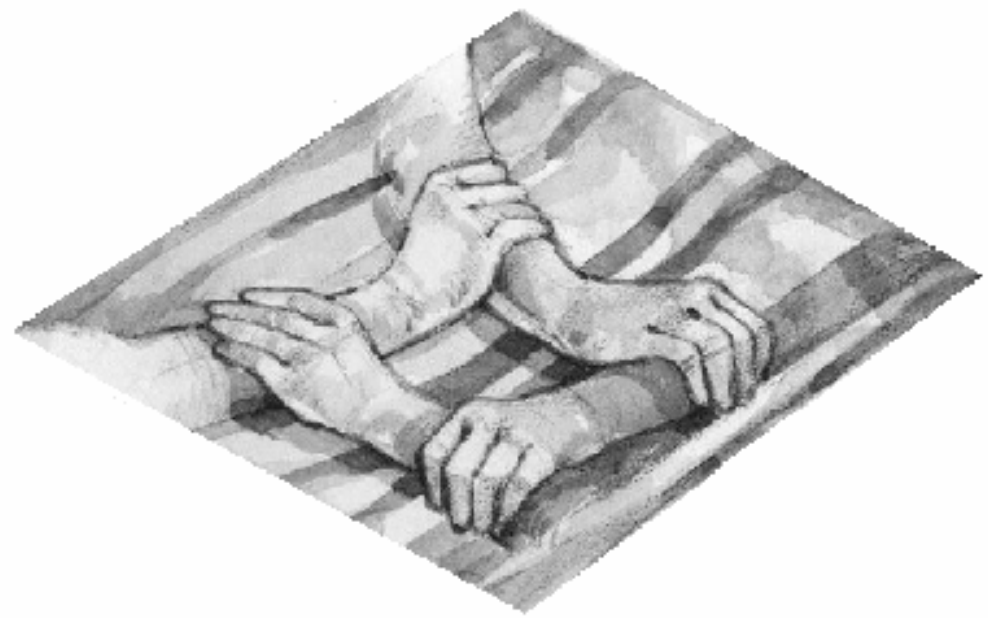




\section{Biography of Dr. Salah Osman}

I met Dr. Salah Osman a year ago by Internet. He is a passionate researcher in the Philosophy and History of Sciences and in Symbolic Logic, while I did research in mathematics and multivalued logic. Therefore, we got a common point of study and research in using Neutrosophic Logic (which is a generalization of Fuzzy Logic) and Neutrosophy (which is an extension of Dialectics) in Arabic and more general Muslim Philosophy.

His interests also comprise Research Methods, Philosophy of Language, and Philosophy of Chemistry.

Dr. Salah Osman was born in Alexandria, Egypt, in 1963. He is married and has three children.

Dr. Salah Osman was educated in Egypt, received his B.A. from Alexandria University in 1985, and his M.A. from the same university in 1993 with the thesis Reason and Dialectics in the Philosophy of Herbert Marcuse. His Ph.D. from the Department of Philosophy of the Faculty of Arts at Minufiya University in 1996, with a thesis on The Problem of Continuity and Infinity in Philosophy, Physics, and Mathematics (with first class honors).

He started working as a Demonstrator in the Department of Philosophy of the Faculty of Arts at Minufiya University in Egypt between 1989 and 1993, then Assistant Lecturer between 1993 and 1996, Lecturer between 1996 and 2003, and Assistant Professor since 2003 at the same university. During the academic year 20042005, he served as a Department Chair.

He has published many books, such as:

- Continuity and Infinity between Science and Philosophy (AlItisal wa al-Latanahi bayna al-Ilm wa al-Falsafa), The Establishment of Knowledge (Munsha't al-Ma'arif), Alexandria, Egypt, 1998.

- Scientific Model between Imagination and Reality (Al-Namuzaj al-Ilmi bayna al-Khayal wa al-Waqi'), The Establishment of Knowledge (Munsha't al-Ma'arif), Alexandria, Egypt, 2000. 
- Darwinism and Human: The Theory of Evolution from Science to Globalization (Al-Darwiniyah wa al-Insan: Nazariyt alTatwur menn al-Ilm ila al-Awlama), The Establishment of Knowledge (Munsha't al-Ma'arif), Alexandria, Egypt, 2001.

- Many-Valued Logic between Degrees of Truth and Limits of Knowledge (Al-Mantiq Mut'adid al-Qiyyam bayna Darajat alSidq wa Hudud al-M'arifa), The Establishment of Knowledge (Munsha't al-Ma'arif), Alexandria, Egypt, 2002.

- Towards a Philosophy of the Chemistry (Nahwa Falsafa li alKimya'), The Establishment of Knowledge (Munsha't alMa'arif), Alexandria, Egypt, 2004.

- The Illusion of External World between Language and Cognition (Wahm al-Alam al-Khariji bayna al-Lugha wa al-Idrak), The Establishment of Knowledge (Munsha't al-Ma'arif), Alexandria, Egypt, 2004.

- The Nature of Spatial Boundaries between Geography and Philosophy (Tabi'at al-Hudud al-Makaniyya bayna alJughrafiyya wa al-Falsafa), The Egyptian meeting for invention and growth (Al-Multaqa al-Misri li al-Ibda' wa al-Tanmiyya), Alexandria, 2005.

- Color realism: Reading in the Essence of Color and the Ways of Consciousness of it (Al-Waqi'iyya al-Lawniyya: Qira'ah fi Mahiyyat al-Lawn wa Subl al-Wa'iy Bihi), The Establishment of Knowledge (Munsha't al-Ma'arif), Alexandria, Egypt, 2006.

And many articles:

- The Tree of Universe and Counterfactuals in Storrs McCall (Shajarat al-Kun wa Qadaya Munaqadat al-Waqi'), The Journal of Faculty of Arts, Minufiya University, Egypt, (39), October 1999, pp. 83-128.

- Semantics of Indexicals and Indirect ( Reported ) Speech (Semantiqa al-Mu'shirat al-Lafdhiyya wa al-Kalam Ghayr alMubashir), The Journal of Faculty of Arts, Minufiya University, Egypt, (46), July 2001, pp. 127-166.

- Science, Philosophy, and Religion as Categories for Raising of Arabic mind (Al-Ilm wa al-Falsafa wa al-Din ka Maqulat li Nahdat al-Aql al-Arabi), The service Center for Research 
Consulting, Division of Translation, Faculty of arts, Minufiya University, Vol. 15, March 2003.

- The Dialectic of Constancy and Motion in Zeno's Paradoxes: a Contemporary Mathematical View (Jadal al-Thabat wa alHaraka fi Mufaraqat Zenon), Journal of Facult of Arts, Menoufia University, Egypt, (58), July 2004,pp. 99 -139.

Dr. Florentin Smarandache

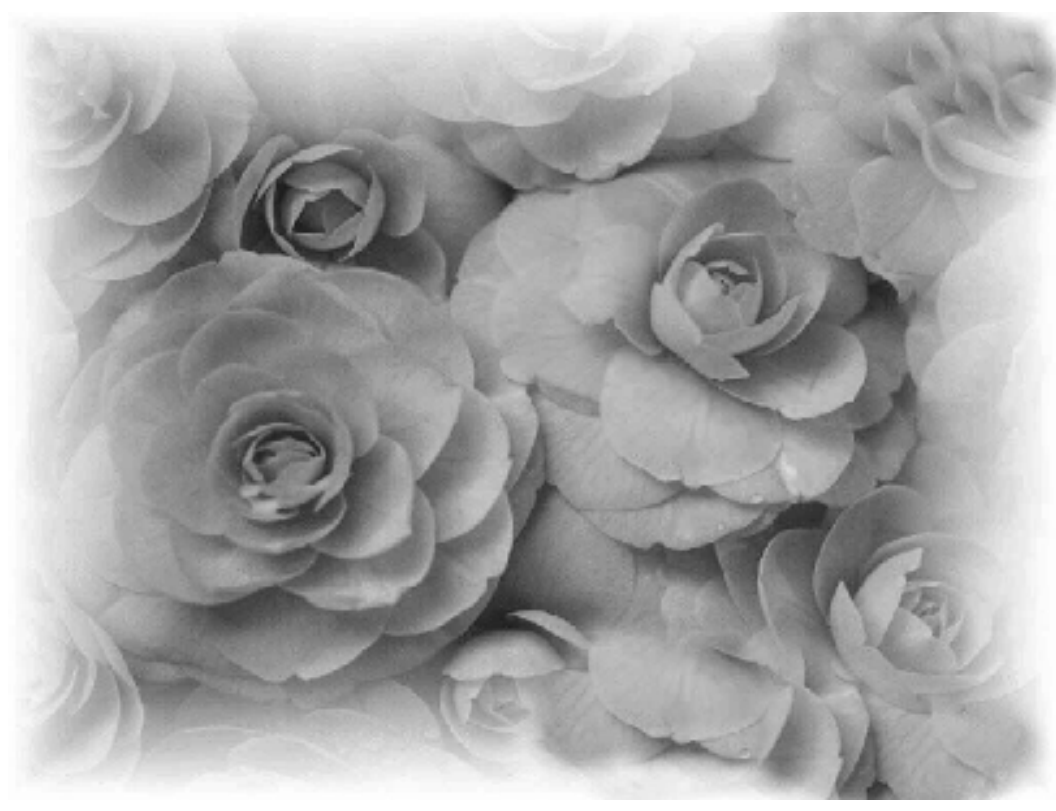


Neutrosophy is a theory developed in 1995 by Florentin Smarandache as a generalization of dialectic. This theory considers every notion or idea $<A>$ together with its opposite or negation < antiA $>$ and the spectrum of "neutralities" < neutA> (i.e. notions or ideas located between the two extremes, supporting neither $<A>$ nor $<$ antiA $>$ ). The $<$ neutA $>$ and $<$ antiA $>$ ideas together are referred to as $<$ nonA $>$. In this theory every idea $<A>$ tends to be neutralized and balanced by $<$ antiA $>$ and $<$ nonA $>$ ideas - as a state of equilibrium.

Hence, neutrosophy is based not only on analysis of oppositional propositions as dialectic does, but on analysis of these together with neutralities in between them as well.

Neutrosophy was extended to Neutrosophic Logic, Neutrosophic Set, Neutrosophic Probability and Neutrosophic Statistics, which are used in technical applications.

In the neutrosophic logic every logical variable $x$ is described by an ordered triple $x=$ (T, I, F), where $T$ is the degree of truth, $F$ is the degree of false, and $I$ the degree of indeterminacy, with $T$, I, F subsets of the non-standard unit interval $]^{-0}, 1^{+}[$. In addition, these values may vary over time, space, hidden parameters, etc.

Examples of Neutrosophy used in Arabic philosophy:

- While Avicenna promotes the idea that the world is contingent if it is necessitated by its causes, Averroes rejects it, and both of them are right from their point of view.

Hence $<A>$ and $<$ antiA $>$ have common parts.

- Islamic dialectical theology (kalam) promoting creationism was connected by Avicenna in an extraordinary way with the opposite Aristotelian-Neoplatonic tradition. Actually a lot of work by Avicenna falls into the frame of neutrosophy.

- Averroes's religious judges (qadis) can be connected with atheists' believes.

- al-Farabi's metaphysics and general theory of emanation vs. al-Ghazali's Sufi writings and mystical treatises [we may think about a coherence of al-Ghazali's "Incoherence of the Incoherence" book].

- al-Kindi's combination of Koranic doctrines with Greek philosophy.

- Islamic Neoplatonism + Western Neoplatonism.

- Ibn - Khaldun's statements in his theory on the cyclic sequence of civilizations, says that:

Luxury leads to the raising of civilization (because the people seek for comforts of life) but also Luxury leads to the decay of civilization (because its correlation with the corruption of ethics).

- On the other hand, there's the method of absent-by-present syllogism in jurisprudence, in which we find the same principles and laws of neutrosophy.

- In fact, we can also function a lot of Arabic aphorisms, maxims, Koranic miracles (Ayat Al-Qur'ãn) and Sunna of the prophet, to support the theory of neutrosophy.

Take the colloquial proverb that "The continuance of state is impossible" too, or "Everything, if it's increased over its extreme, it will turn over to its opposite"!

ISBN 1-931233-13-6
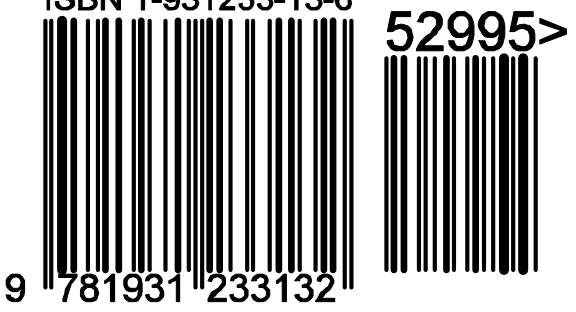